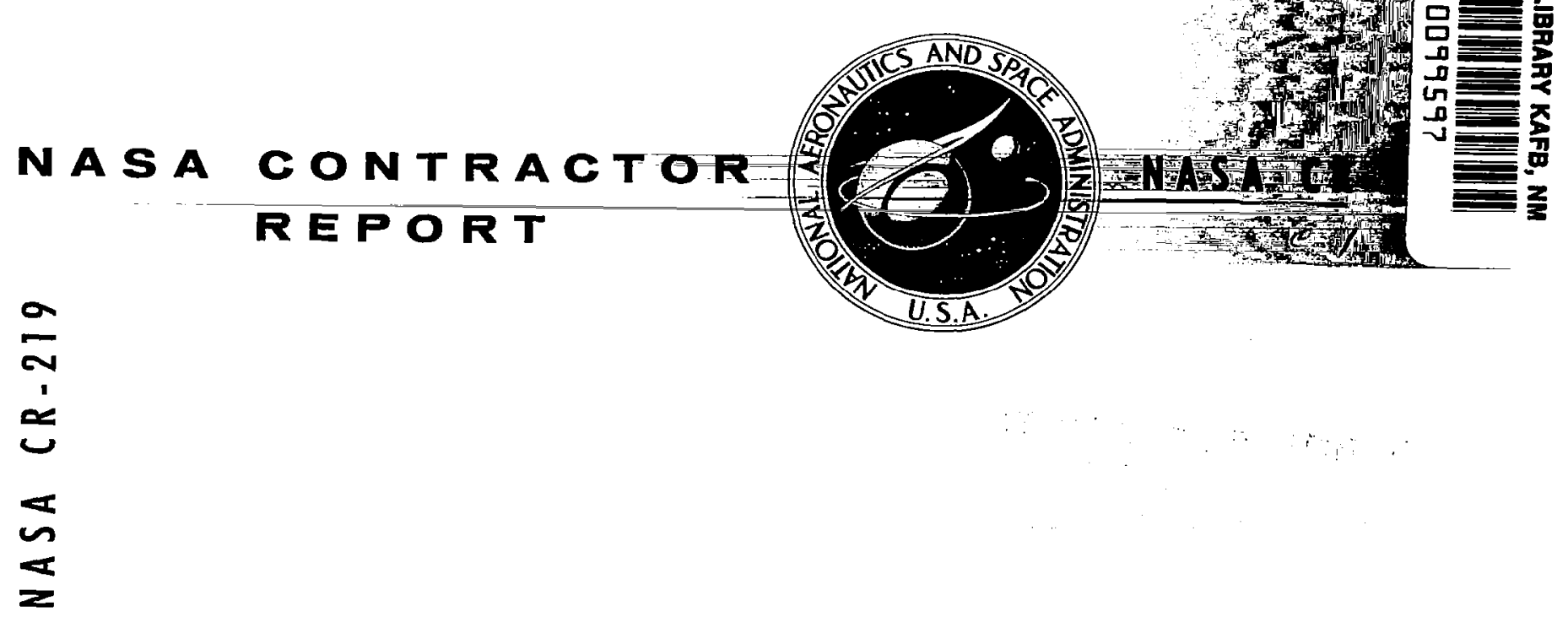

\title{
FURTHER STUDY OF THE \\ DYNAMIC SYSTEMS RESPONSE OF SOME INTERNAL HUMAN SYSTEMS
}

by A. S. Iberall and S. Z. Cardon

Prepared under Contract No. NASw-1066 by GENERAL TECHNICAL SERVICES, INC. Yeadon, Pa.

for

NATIONAL AERONAUTICS AND SPACE ADMINISTRATION • WASHINGTON, D. C. • MAY 1965 
NASA CR-219

FURTHER STUDY OF THE DYNAMIC SYSTEMS RESPONSE

OF SOME INTERNAL HUMAN SYSTEMS

By A. S. Iberall and S. Z. Cardon

Distribution of this report is provided in the interest of information exchange. Responsibility for the contents resides in the author or organization that prepared it.

Prepared under Contract No. NASw-1066 by GENERAL TECHNICAL SERVICES, INC. Yeadon, Pa.

for

\section{NATIONAL AERONAUTICS AND SPACE ADMINISTRATION}

For sale by the Clearinghouse for Federal Scientific and Technical Information Springfield, Virginia 22151 - Price $\$ 5.00$ 
- 
This report has been taken from material in a Third and Fourth Quarterly Report prepared under contract No. NASw-678, dated April 1, 1963. The first two quarterly reports have recently been published $(1,2)$. In the first report, control in biological systems was reviewed. In the second report, a theoretical and experimental study was begun on four internal systems. The present report continues study of these four systems. A preliminary section makes a contribution toward a new theory of automatic control as a background for analysis of the entire biological system. 


\section{TABLE OF CONTENTS}

$\underline{\text { Page }}$

SUMMARY $\ldots \ldots \ldots \ldots \ldots \ldots \ldots \ldots \ldots \ldots \ldots \ldots \ldots \ldots \ldots \ldots \ldots \ldots \ldots \ldots \ldots \ldots$

INTRODUCTION $\ldots \ldots \ldots \ldots \ldots \ldots \ldots \ldots \ldots \ldots \ldots \ldots \ldots \ldots \ldots \ldots \ldots \ldots \ldots$

I. GENERAL DYNAMICS OF SYSTEMS, AND ITS RELATION WITH A

NEW THEORY OF AUTOMATIC CONTROL ..................... 8

A. Preliminaries $\ldots \ldots \ldots \ldots \ldots \ldots \ldots \ldots \ldots \ldots \ldots \ldots \ldots \ldots \ldots \ldots \ldots$

B. Review of Current Status of Control Theory ........... 12

C. Toward a New Control Theory ..................... 20

II. DYNAMICS OF THE THERMOREGULATION SYSTEM .............. 29

A. Theoretical Dialogue Continued .................. 29

B. Oscillatory Temperature State of the Human $\ldots \ldots \ldots \ldots \ldots \ldots$.... 41

C. Further Investigation Into Temperature Dynamics ........ 45

III. CARDTOVASCULAR SYSTEM DYNAMTCS $\ldots \ldots \ldots \ldots \ldots \ldots \ldots \ldots \ldots \ldots \ldots \ldots$

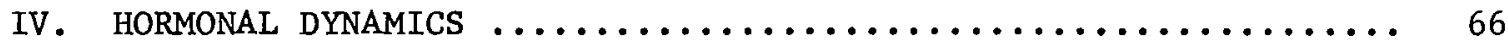

V. BEHAVIORAL ORGANIZATION $\ldots \ldots \ldots \ldots \ldots \ldots \ldots \ldots \ldots \ldots \ldots \ldots \ldots$

A. Speculative ............................ 73

B. Review and Commentary Continued $\ldots \ldots \ldots \ldots \ldots \ldots \ldots \ldots \ldots$

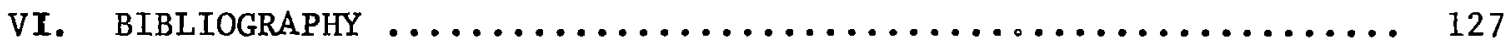

VII. ACKNOWLEDGEMENT .............................. 132 F IGURES 


\section{SUMMARY}

effort.

This project has succeeded in six major vantage points in one year of

1. Temperature regulation. It has been shown that both the ventilation flux (the minute volume), as a measure of metabolism, and the surface temperature potentials are oscillatory with a spectrum of periodicities similar to what was described in earlier studies. The evidence that this arises from engine and other internal oscillator cycles is increasingly strong. Direct evidence is produced that heat sources likely lie in the muscle layers by showing that the muscle layer is hotter than its neighboring layers. A background of flux and potential characteristics, both static and dynamic, now exist in a consistent physical description, for the nude human in the temperature range $10-40^{\circ} \mathrm{C}$. The problem now awaits detailed physiological demonstration of the mechanisms that create this static and dynamic regulatory picture. Some of the likely elements are alluded to.

2. Cardiovascular regulation. As a first physical problem, the mechanica1hydrodynamic events in the cardiovascular system, mainly in the arterial system, has been explored. The conclusion has been drawn that the sequence of quasi-static events in the system are as follows: a pulse of flow is pumped by the heart into the aorta before the heart valve shuts; the radial elasticity of the aorta and of other arteries transforms this pulse of flow into a ramp of pressure; the flow resistance of the capillary-arteriole vascular hed allows the stored volume to leak out at essentially a constant rate with an attendant decay of pressure. This is the 'windkessel' model of otto Frank. This study has demonstrated that this is the essential characteristic of the entire arterial system, which more modern discussion of pulsatile events in the system has befogged. The additional dynamic events which are superimposed on the 'windkesse1' response characteristics, leading only to mild deformation in the actual characteristics, arise from two sources. The pulse of flow into the aorta develops both a longitudinal and a radial response from the aortic system. The longitudinal response likely arises only from volume deformation in the closing valves, rather than in the aorta, because the aorta is nearly 'rubber-like' (i.e. it has a Poisson's ratio very near $1 / 2$, so that longitudinal and hoop strains compensate), and it is longitudinally tethered. The volume deformation likely creates the high frequency (approximately 16 cps) oscillation that is associated with the incisura notch. It is probably viscously damped during its transit of the aorta. The radial elastic strains, coupled with the radial flux of blood possessing liquid-1ike inertia, develops an elastic wave, the Moens-Korteweg wave, that travels down the aortic and arterial walls. This creates the frequency associated with the later dicrotic wave (approximately $4 \mathrm{cps}$ ). This wave is essentially undamped in the aorta, the larger arteries, and even the moderately large ones. Finally in sufficiently small arteries, it is damped out viscously. This wave does not begin to make itself very evident till the signal has traversed into the arterial system and begins to resemble a small standing wave. While most of the ingredients of the model have been available, what is novel in their assembly is the idea that the modern pulsatile transmission characteristics are just a moderate 
deformation of Frank's piece-wise concept of cardiac cycle events, rather than Womersley's unified pulsatile transmission concept, and that the 'higher' frequency characteristics (higher than the heart beat and flow pulse) arise from two independent sources, the longitudinal compliance and the radial compliance.

3. Hormonal system. A conceptual start of considerable biological significance was made for viewing the dynamic action of hormones. The concept likely has much greater significance than any hormonal experiments that have been begun. If the internal systems of the body are regarded as oscillators, then the effect of hormones, as chemical mediators of such oscillator cycles, should make itself evident by the concepts of spectral or frequency analysis common to al1 other 'spectroscopic' techniques. Listing a fair number of oscillator systems (a dozen or so) which provide indicators at various time scales from hundredths of a second to a few years for detectors of the trace of a hormone, two oscillators were chosen for some preliminary exploration, because of their easy experimental time scale. These were the heart beat rate, and the ventilation flow rate. Equilibrium measurements showed a similar one to two minute cycle in heart beat as had been found in ventilation rate and heat flux rate. This is considered preliminary evidence of a hormonal - not a gas exchange cycle. Further it was believed that it might be possible to follow hormonal transients down to the ten seconds level and up to the 5 hours level with just these two cyclic indicators. Preliminary data (on a dog) has sought the transient effects, directly into the blood, of bicarbonate, adrenaline, insulin, hydrocortisone, vasopressin, ACTH, parathyroid, thyroxin, and acetylcholine.

4. General systems dynamics. The problem posed of decoding the biological system, namely of finding a physical foundation, other than the general laws of physics and chemistry, for viewing the dynamic complex that is the macroscopic human animal, has led to a new concept of the regulation and control dynamics of physical systems in general. The central formulation of control theory, as a general problem in non-linear mechanics, is to analyze and synthesize those physical systems that can be represented or designed for within a narrow slit perpendicular to the 'displacement' axis in phase space, regardless of disturbances, both static and dynamic. (This definition is for the simplest case, the analogue of the positional servo). This formulation is meant to replace the formulation of comparison of output with a reference at an error summing node, and the operational amplification of the error to take corrective action. The former is more general, and includes all dynamic problems that are associated with regulation and control. The brevity of formulation should not be mistaken either for naivete or simplicity. (For example, the general physics of dynamic systems may be characterized as dealing with the exposition of systems that can be represented anywhere in the phase space. Control systems are only moderately more restrictive). Characteristic behavior that emerges is the dynamics and stability of both static and dynamic regulators; control dynamics both as a near-linear decay toward the control equilibrium, or as a nonlinear limit cycle oscillation, as in 'bang-bang' control.

5. Biological systems dynamics. The exploration of the dynamic characteristics of the biological system, of the fundamental role of homeostasis, and of limit cycle oscillators, particularly for regulation purposes in the biological system, led to a much broader generalization that all internal systems in the 
biological system consist of limit cycle oscillators, and that the system is governed both chemically and electrically by moderating the stability of these oscillators. It was this generalization that led to a view of general control dynamics of all physical systems. This concept is perhaps best described as 'homeokinesis', as a modification of Cannon's term 'homeostasis'.

6. Behavioral system dynamics. In an attempt to measure this concept of biological system dynamics against the ideas expressed by well known investigators in the field of mental processes, it was surprising that these ideas had been most nearly anticipated in concept - perhaps not in methodological detail - by Sigmund Freud. It is proposed that the summation of 'hungers', and system drives that Freud identified as the ego make up a system of loosely coupled oscillators; the governing complexes of super-ego and id also represent oscillator complexes; the logic contained in such adaptive oscillator complexes, it is hoped, will provide a sufficient complex, open, logical system to account for human behavior as a computer complex. The latter idea is proposed as a substitute for current digital computer logics based on essentially Boolean algebra, or for the elementary concept of feedback which is commonly misapplied outside of the field of control engineering. It is hoped that this key idea of oscillator complexes in non-linear mechanics will bridge the foundational problems in Adrian neurophysiology, Freudian psychoanalysis, Pavlovian conditioned reflex concepts, Hull behaviorism, and Wiener cybernetics, with the aid of a liberal besprinkling of Van der Pol oscillators.

A major property that arises from these oscillator complexes is the nonlinear property of 'synchrony' (or synchronization) in which a non-linear coupling link both systems together by what may be best described as weak but persistent coupling forces. This common locking-in of orbits between people, parent-child, etc., may be perhaps described as orbital synchrony. (Two hearts that beat in $3 / 4$ time is its 1iterary sense). 


\section{INTRODUCTION}

This program has been devoted to examining regulation and control in four internal systems in the human - the core temperature regulating system, the cardiovascular system, the hormonal system, and the behavioral system - from a physical point of view. Because of the state of the art of the problem in each field, there were considerable restrictions on what phase of each problem could be most fruitfully attacked by physical methodology. Thus each topic has developed in its own course.

In the first report (1), a general view of homeostatic regulation was discussed, and the system was then reviewed from the point of view of its salient autonomous oscillators.

In the second report (2), an experimental program designed to exhibit the static and dynamic surface temperature potentials and ventilatory or metabolic fluxes was reported on. The static characteristics were tied into known data in the literature to establish a valid basis for the dynamic data. Discussion was continued to attempt to lay a foundation for the dynamic processes by which the core temperature regulation takes place.

The cardiovascular literature was reviewed for material on the hydrodynamics of blood flow and pressure in the arterial and venous systems. An elementary hydrodynamic model was presented as background. It was concluded that the two systems as a whole each acted substantially like the Frank "windkessel" model of an elastic tube and a resistive capillary bed. This left the role of the Moens-Korteweg elastic aortic transmission wave, that has been more recent1y popularized, ambiguous, particularly since sufficient damping could not be found to prevent appreciable resonance.

Literature on hormones was reviewed to provide a firm summary on which to base a physical exploration for the dynamics of the hormones.

Finally, a review and commentary was begun on behavioral ideas to seek out a foundation for a computer mode1 of behavior.

In this third report, these studies are continued. The dynamic data on temperature and ventilation are presented. Experimental evidence is brought forth that the body's power source resides in the muscle sheath distributed over the body. Further study of the cardiovascular data has led to a reconciliating hypothesis to place the roles of both the over-damped windkessel model, and the sources of higher frequency pressure and flow oscillation in perspective. A purely physical program has been hypothesized for viewing hormonal action, and some preliminary experimental data have been assembled.

A general model of the biological system has developed, and it is proposed as a broad organizing hypothesis. The same general biological systems model has developed hypothetically as applicable to the behavioral system.

A key concept of control systems theory has emerged from this work. If the view developed is accepted, it may capture a major hope for the so-called 
bionics program of finding a clue to regulation and control theory in the biological system. Thus, a degree of physical unity has arisen for viewing the biological system that was not at all evident when this study began.

At the time this program started, an invitation was received to prepare a general review and commentary of regulation in biological systems. This seemed to fit well within the scope of the present contract, and so such a general review was developed (1). Two lines of study, which were pursued purely as a matter of logical organization have now loomed into crucial biological significance.

1. Oscillators within the biological system. While still preserving what may be a controversial position on automatic control theory, in order not to mislead the biological reader, a specific frame of reference was proposed for the analysis of any dynamic system and applied to the biological system. A particular view was discussed of the difference between regulation and control. A general line of physical analysis, consistent it is believed with such classical mechanics texts as whittaker (3), was developed. The first step, beyond recognizing the existence of bounded mean states of a system was to explore any intrinsic oscillators at work before searching for regulating or controlling action. The study found so many oscillators that it never really got much beyond these clements.

2. Relation between homeostasis and system dynamics. The main descriptive biological line pursued was a thread from Cannon's homeostasis, (i.e. regulation in the terms of (1))to many sustained oscillating systems inside the biological system. Although the ideas of homeostasis and oscillators might appear to be disconnected, there were two reasons for attempting such a bridge. First, a physical science program newly coming to the biological system was not going to make any immediate significant contribution to the understanding of the general physiological structure and function of biological systems, whereas it could uniquely contribute help in understanding the dynamics of the systems. Second, previous work in a variety of fields, biological (4, 5, 6, $7)$, and non-biological $(9,10)$ has been subconsciously pursuing and building up a scientific structure for connecting regulation to system dynamics.

For its second report (2) the program had to get down to the beginning details of a dynamic study of the temperature regulating system, a review of hormonal literature to uncover whatever dynamics could be found on the dynamic action of hormones preliminary to an experimental investigation, and at least speculation and review of the dynamic organization of the behavioral complex (which it proposed to view as a computer-governor-communications network). Most of these problems began by classic (in physical sense) research routines and got the program off to a satisfactory start. However, the section that reviewed the dynamics of hormones appeared slim in ideas. It appeared that the investigators in this field, barring a very small number of isolated cases, had not begun any real modelling of the dynamics. The experimental difficulties were enormous. The only path that seemed immediately available was one that appeared to be a trap for physical scientists, in that it would involve a lifetime of detailed work with no over-all broad physical conclusions by such methods as bioassay, or detailed chemical analysis for inference of hormones. Therefore, a substitute was sought in reviewing the program after finishing the second report. 
A new idea developed, which seemed to be a Gestalt of al1 these elements that had gone before. Briefly, this idea had the following content:

1. The many fold oscillators in the biologic system (which, repeated1y emphasized in (1), emerge as autonomous 'engine' cycles) are not incidental characteristics of the biological system. These intrinsic oscillators are the biological system.

2. Cannon's entire concept of homeostasis (which, as was repeatedly emphasized in (1), emerges as a regulation characteristic) is obtained as a result of shifting the stability of these intrinsic non-linear oscillators. There is likely no other way that the system has for operation, and thus in accordance with (1), this may best be described as an illustration of dynamic regulation.

3. It emerges, as a very important hypothesis, that the same type of instability characteristic is the foundation for all automatic control theory, and as such represents a new foundation and a new type of automatic control theory.

4. In that case, 1ike in all 'atomistic' systems in which the 'atomic' element is an intrinsic oscillator, its systematic analysis can be accomplished by a steady state and transient 'spectroscopy' of its intrinsic oscillators. A reasonable prediction, therefore, is that the biological system will, in general, be decoded by a 'spectroscopic' study (i.e. frequency response, and phase plane and phase space topological study of its limit cycles. For an introductory background see (11))of the steady state and transient response of its intrinsic oscillators.

(While application of these ideas will be discussed and illustrated in subsequent sections, it is worthwhile to note that during this period of gestation of ideas, it was possible to propose application of these ideas to other biological research. At a local January 1964 Cleveland meeting of the Professional Group on Medical Electronics of the AIEEE, Dr. Mathew Levy: discussed work that he is doing in attempting to elucidate the isolated action of the baroreceptorin the carotid sinus in controlling the vigor of the contraction of the heart. This work, originated by Sarnoff at NIH, and also pursued by Salisbury, involves pumping and interconnection of test animals and external sources to attempt to follow purely the isolated action of pressure changes in the carotid sinus in the relations of carotid pressure and exit stroke volume from the heart. Each of these investigators has developed a complex experimental situation to probe at these ideas. In answer to a general request by Levy for criticism, we pointed out that in accordance with the 'windkessel' exposition in (2) there was little intrinsically interesting information contained in the relation of 'output' flow to 'input' pressure, since the pressure and flow were already physically connected by resistancecapacitance relations. The only fundamental way that we could see for getting at the desired control relations was by a phase plane analysis of pressure (say at the carotid sinus) versus the time derivative at exit pressure (say from the heart) during each cycle. It is in such an analysis, cycle by cycle, rather than changes in mean cycle performance, that the non-linear control 
cycles would make themselves evident, and indicate the likely nature of the baroreceptor control (whether of level, derivative, combination, or more complex, including non-linear, combinatory signal). Such a philosophy of analysis is not well known in biological research, although predominance of both factors, of quantity and rate, is significantly recognized in a number of problems.).

With this introduction, it is now useful to turn toward more detailed exposition and application of these ideas in some specific cases of interest in this program. 


\section{THE GENERAL DYNAMICS OF SYSTEMS, AND ITS RELATION WITH A NEW THEORY OF AUTOMATIC CONTROL}

\section{A. Preliminaries}

As a scientific background, consider the general technique of dynamic systems analysis, which can only be very briefly outlined here. (It was also partially sketched in (1).).

1. Identify generalized displacements in a physical system. (One knows that one is dealing with physical displacements in a system - as opposed to say mathematical or literary 'displacements', or any other nonphysical 'displacements' - if one can find a conjugate quantity known as a generalized 'force', such that the product represents physical energy

$$
Q \mathrm{dq}=\mathrm{dE}
$$

$\mathrm{Q} \quad=$ generalized force

$\mathrm{dq}=$ generalized displacement increment

$\mathrm{dE}=$ an increment of real physical energy

that can be detected, say, as a change in temperature in a mass in a calorimeter, or as a transit gradient of temperature in material at the boundary of a calorimeter.)

2. Identify the conjugate forces.

3. In systems of complete specification, comb through all possible displacement types (which will become identified as the total number of degrees of freedom of the system) for completeness and for redundancy.

4. Arrange these dual elements (of forces and displacements) by some generalized physical scheme, for example by the Lagrangian program (which is not a purely mechanics program, although its usual exposition is commonly given in mechanics. It is easy to note the development of the program for lumped electrical entities - inductance, current, electric charge, electric potential, etc. - by the Lagrangian scheme. It is more difficult to note, for example, in the elegance with which Fermi brought the electromagnetic radiation field into the Lagrangian scheme).

5. The result of the generalized physical program, at least for continuous systems, leads to a set of simultaneous differential equations, one for each degree of freedom, or generalized coordinate (or, as the number of degrees of freedom approach infinity, by a partial differential equation set, with one equation for each primary field variable distributed in space as well as time). This sort of program is extremely well illustrated in such a classic as Whittaker (3).

6. What remains, as mathematical detail, is the solution of these sets. This is no mean task, and likely represents the main bottleneck in the 
exposition of the dynamics of systems. (This may be contested by many physicists, in particular theoretical physicists. Yet, in a fashionthat they may not be willing to accede to, it is true. The elementary ideas of physics are quite good - even if fundamental particle and field physics is in trouble. Yet, before one gets too far in the physical program, the mathematical bottleneck arises, and the theoretical physicist commonly walks away from the problem. As illustrations: in (3) the properties of the integrals of dynamic systems are discussed, the three body problem and the general orbital problem is sketched out and the book ends; in (10) the mechanics of continuous media in this case the $n$ - body problem of the hydrodynamic properties of an ensemble of molecules is sketched out in great detail, but the Navier-Stokes equations are still unsolved; some formulation is possible of the equations of elasticity-plasticity for solid bodies but no wide range, high quality solutions - other than linear Maxwell or Voight models - exist; there are also similar unresolved difficulties on the electromagnetic, molecular, atomic, and nuclear levels, but this is not a suitable forum in which to begin controversies in these fields). The common thread that likely runs through all of these problems is a lack of mathematical apparatus for dealing with the very difficult non-linear problem. (A background for the linear problem is contained in such excellent treatises as Forsyth (12), Ince (13), and Poole (14).) This point of view has formed from working diligently in non-linear mechanical problems for the past two decades, and trying most earnestly to interest colleagues in the instruments, regulator, and automatic control field.

7. The most general approach to begin the solution of the physical problems represented by the equation sets is to determine whether there are solutions in which the systems get away (i.e. they take off monotonically and disappear in space, or explode), settle down to rest, or most interestingly form periodic or conditionally periodic 'orbits'. While this may be a general philosophic view in non-linear mechanics (see for example (11) or (15) ) it becomes truly a difficult problem when either the number of degrees of freedom grows, or the physical program is not well developed. (As a biological illustration of such difficulties, one may view a most recent study (16) on the 'additivity' of dynamic effects in a biological system responding to environmental inputs).

8. Once methods of solutions are available, solutions are then sought for particular boundary conditions of starting, of boundary values, ctc.

This program really is only applied in limited part in automatic control theory. Instead, most commonly a particular linear or near linear artifice is chosen in which the dynamic equations of motion are essentially set up as deviation equations from a particular mean state (not necessarily fixed in time). This will be discussed subsequently.

The automatic control program has been disturbing in that it seemed very artificial to search for an 'error' function, or error summing point in the circuit around which to construct the equations. It would have seemed more to the point, to write as completely as possible, a good set of dynamic equations and then seek out the singular states of motion (in a non-linear sense) in these coupled (the term 'coupled' always seemed more general and fundamentally valid than a particular instance in the concept usually termed 
'feedback') networks. System motion then is defined with regard to these singularities. However suggesting this type of analysis in the field of automatic control always has drawn a lack of interest.

For example, at a 1953 Gordon Conference on Instrumentation, during a pane1 discussion on the control problems of the automatic plant, we began to voice at considerable length serious concern with control description of complex plant processes by linear feedback analysis, and proposed a prov ocative and controversial thought that there was practically no difference between controller and a non-linear limit cycle characteristic (i. e. that to each feedback controller there may correspond a unique non-1inear network). At that time, the idea was only an intuition on our part and we were not completely certain of what it meant in toto. (I1lustratively, at about that time we developed a company study on the stability of a pneumatic regulator, 'reinventing' what has become known as the Kochenburger describing function technique for the investigation. What seemed clear was that a linear analysis of the theory of small vibrations typc - sce (3) - not the linear expansion around an operating point, would only give necessary conditions of instability and that a second fuller non-linear exploration was needed to determine sufficiency conditions. While this paper was unpublished, Tsai and Cassidy (17), at a 1960 ASME Annual Meeting, presented such a study, performed by computer means. While these are not feedback control papers, per se, they really begin to get at the problem difficulties. It is only the total common problem that is not clear). It is likely only now in this report that we can present the full import of this concept in its first preliminary exposition.

A recent opportunity to hear review of the outstanding problems and state of the art that exist and remain in automatic control, appropriately provided material for a brief summary of these problems as a backdrop and introduction to current theory and a new theory. The sources were A. J. Young, Director of the Central Instrument Laboratory of Imperial Chemicals, reviewing in private discussion the state of automatic and computer control as it affects large chemical process industries; and a talk by Harold Chestnut, chief control engineer for General Electric, and author of one of the older outstanding books in the automatic control field, who reviewed the state of control theory in complex process industries at the November 1963 Annual Meeting of ASME. As he put it, "The 1963 JACC and IFAC meetings presented a number of new ideas on automatic control applications and theory", and he proposed to examine them. The reason for referring to illustration from the complex process industries is that applications in this field run a similar gamut of complexity of control and organization as does the biological system, and very often with most similar materials, though other physical-chemical processes. The application to process control very nearly reaches the limiting conceptualizations of control theory today.

These men, in summary, saw these problems:

1. There is a real need to solve automatic control problems with understanding, rather than by any "seat of the pants art". The systems have become too complex and mistakes too costly. 
2. There is a need to solve these problems theoretically. It has become too expensive and complex for piecemeal pilot studies.

3. There is need for a general hierarchical control theory.

4. There is need for economic control with adequate performance, in large number quantities. While elegant elements can be constructed, their expense rules them out in complex systems (shades of the biological entities! Note, here the biologists have been far ahead of the physical scientists in understanding for a long time).

5. The concepts of automatic control today involve:

a) Optimizing control, by various control logic criteria. This, however, is not done for non-linear dynamic systems.

b) Adaptive contro1, for elements, for processes, and as a predictive adaptive scheme. In all of these the problem lies in the structuring of the learning process, and of availability of a hierarchical series of controls.

such as:

6. Today's requirements in very specific terms are "practical" ones

a) soft ware for on-line digital systems

b) soft ware for off-line digital analysis

c) ability to use new components such as fluid amplifiers, and solid state elements

d) quicker, cheaper installation and start-up

and "theoretical" ones such as:

a) theory of automatic control economics (how to design cheaply, etc.)

b) theory of automatic control reliability

c) knowledge of process to be controlled

d) improved modelling methods

e) increased sensitivity to change in systems

In our opinion there is no difference in the theoretical problem of the dynamic analysis of a complex system, or in the control characteristics found or required for mechanical, chemical, biological, guidance, aeronautical, or automotive system. Thus, if one can solve one (if complex), one can solve a11!! Thus, in many respects the discussion will be continued along the parallel tracks of the physical system, the biological system, the behavioral system (i.e. what gives the individual response unique content), and the social system. 


\section{B. Review of Current Status of Contro1 Theory}

Suppose one follows elementary automatic control theory from an older source, Chestnut and Mayer (18).

A simple control problem (p. 79) is identified as,

$$
A \frac{d^{2} c(t)}{d t^{2}}+B \frac{d c(t)}{d t}+K c(t)=K r(t)
$$

$c=a$ controlled variable

$r=a$ reference input, or the driving function

$A=$ effectively, an equivalent mass coefficient

$B=$ effectively, an equivalent damping coefficient

$\mathrm{K}=$ effectively, an equivalent stiffness coefficient

In this view, the controlled variable follows the input variable $r$, with errors $(c-r)$ that depend on dynamic reactive elements. Various methods for describing the response solution are taught - by elementary differential equation solution for a variety of standard inputs (pulse, step, sine), by integral transforms (Laplace, Fourier), or transforms that suit arbitrary inputs (the superposition or Duhamel's theorem), by transforms to the complex plane.

With more than one coupled loop, and a single equation for each loop (as is easily viewable from the laws of elementary mechanics, where Newton's laws are applied in turn to each mass, or from the laws of elementary lumped electric networks where Kirchoff's laws are applied in each loop), reduction of the system (usually linear) leads to a single higher order equation such as

$$
A \frac{d^{n} c}{d t^{n}}+B \frac{d^{n-1} c}{d t^{n-1}}+\cdots+K c=K r
$$

or, there may be "integrating" terms, leading to

$$
A \frac{d^{n} c}{d t^{n}}+\cdots+K c=k r+\cdots+a \frac{d^{m} r}{d t^{m}}
$$

The problem is then to characterize the nature of the response (by the techniques described) or the stability of solutions (i.e. Whether they settle down, oscillate, or diverge).

A competently trained classical physicist can find little to get excited about in the solution of such simultaneous linear equation sets, or single high ordered linear equations, different from 'real' difficult problems in classical physics, except development of useful techniques that will cut down the considerable labor that usually may be involved. From a physical sense one would have to describe the control problem as 'trivial'. It is not altogether so trivial when various synthesis criteria are involved, in which a given network, and its corollary response, are supposed to be transformed to 
look like another network, or its corollary response, by the addition of 'control' elements to the networks.

It is thus appropriate to note the latest points of view of control, say as illustrated in recent JACC (Joint Automatic Control Conferences), or IFAC (International Federation of Automatic Control Congress).

For a transition to more current material, following (18) whose first volume was written in 1951 and revised in 1959, one might first view Popov (19), who wrote a Russian edition in 1956, which was translated in 1962.

closed automatic systems include on-off switches; systems giving automatic response to specific inputs; automatic machines and plants; remotecontrol systems; regulators; servos (automatic pilots, tracking, etc.); mathematical computers; compensated measuring elements; synchros. They represent two classes of system - automatic machines carxying out single or repeated operations that do not depend on time; automatic systems which hold a physical quantity in some controlled process over a long period of time. The book is devoted to the second class of systems. They are divided into open and closed loop automatic systems. The open loop system is illustrated as an input transducing device and a controlled output, which may also have a measuring element to permit noting the state of the controlled object. The functioning of the system is not directly dependent on the result of its action.

Connecting the output measure back to the input measure so that the magnitude of action on the controlled object will depend on the deviation of measure in the controlled object from a required value defines closed loop control. All forms of closed loop automatic system may be reduced to one general feedback circuit. As an illustration, an external time dependent perturbing force acts on the controlled object or system. An external time dependent driving signal, or "force", acts on the control system or regulator. It is required that the controlled object respond solely to the external driving signal, and eliminate as far as possible the effect of the external perturbing force on the controlled object.

If the system is a regulator, the external driving force is brought to bear by a setting. Substantial external perturbing forces, such as load or demand, act to disturb the output. Regardless, the output should remain nearly at the set value. (This describes a zeroth order feedback control network).

"A11 other forms of closed automatic systems reduce to these two: .. servomechanism, or ... automatic regulation system" (It is these two that were distinguished in (1) as controllers and regulators; both distinct from a balancing chain.).

In a servo there is a forward circuit from input to controlled object and a feedback circuit from output to input. The output $x(t)$ is supposed to reproduce the input $y(t)$, or operators on $i t$, such as 


$$
x(t) \curvearrowright k_{1} y(t)+k_{2} \frac{d y(t)}{d t}+k_{3} \int_{0}^{t} y(t) d t
$$

An automatic control system is described by means of automatic regulation systems, (these distinctions are faithfully reproduced from $\mathrm{p}$. 10-12 in Popov) which are defined as serving to maintain prescribed values of one or more regulated variables over a long time for arbitrary perturbing forces. The automatic control system consists of "regulated objects and controls". With a single regulated quantity and object and single regulator, the automatic regulation system is called isolated. With several regulators or objects, the automatic regulation system is termed coupled. If several independent regulators are connected to a single object, they are independent isolated automatic regulator systems. "The first automatic regulator in the world .... was invented by ... Palzunov in $1765^{\prime \prime}$, a water level regulator. (Whether Russian or not, this description is misleading. It either does not define "control", or it can be only considered consistent, if by control is meant quasi-static regulation in our terms, or zeroth order feedback control in servo theory classification. The distinction between servos, and "automatic control" systems which are identified in two places as automatic regulation systems - p. 6, p. 10 - bears this out.) Thus in the main, this book deals with the dynamics of regulators, and to a much lesser extent with dynamics of servo systems. In this it is quite different from American philosophy which is concerned mainly with the dynamics of "controllers", namely devices which attempt to reduce the dynamic time dependent error to zero, not the quasi-static regulating error. This is best borne out by study of section 17 in (19) on the History of the Theory of Automatic Regulation. Popov states that the theory of automatic regulation is due to Vyshnegradskii in 1876, although the first automatic regulator - a water level regulator was invented by Palzunov in 1765. In the references in the back there is a hint that Clark Maxwe11 may have also been involved in the theory of automatic regulation. Nowhere is Watt's steam governor, of about 1769, mentioned. The latter two are considered by Western sources to be the beginnings of automatic regulation. The next reference is to Liapounov's work in 1892, which together with Poincare's 1879 thesis and other papers by Poincare, treats of a theory of stability for differential equations, and thus for the motion of physical systems. References to the work of Chebyshev on the centrifugal governor, Stodola on steam turbine regulation, and Zhukovskii on the regulation of machines, etc., all indicate that a theory of regulators, in particular the dynamics of regulators, is the subject being considered rather than a book on automatic control dynamics. This is borne out in that the ideas of control are covered by the American term of servomechanism.

In skimming the book, one will therefore note much that is essentially non-linear mechanics, rather than control theory. In other words, the book comes closer to dealing with the problem of general system dynamics than control theory, with the specific limitation that the points of stability have really certain desired "regulation" characteristics. 
(This description explains something that was quite puzzling to many of the Americans present at the first visit of a Russian regulation and control group in recent years at the April 1958 conference of the Instruments and Regulators Division of ASME. These men were essentially interested in automatic regulation of processes and machines, whereas the American concern was with controllers. The meeting of minds then was at cross-purposes, which straightened out in a few years. This book likely reflects this changing point of view. We have no quarrel with this book. In fact, it illustrates quite well many applications of dynamic analysis, in particular, considerable emphasis on non-linear dynamic analysis of the class of quasi-static elements known as regulators. As such, it is an excellent introduction to the dynamics that might be associated with homeostatic mechanisms and the problem of stability that might be associated with such regulating systems. However, it does not deal very completely with the control problem, or within its frame of reference, with the general dynamics of servomechanisms. Thus in summary, as far as the dynamics of regulators, this book is quite excellent; as far as the statics of regulators are concerned, it contributes very little, and in fact there are very few books that contribute much to this subject, if often being considered a trivial problem, which it is not; as far as control theory is concerned, the amount of material is limited.)

A large scale canvass of automatic control may be viewed in the first IFAC Congress Proceedings from Moscow, 1960, (20). The papers presented at this Congress, issued in four volumes, are quite learned. For review, our inclination is to touch only on a very few that may have some pertinence for the present inquiry. For example, of interest are some of the papers on relay circuit theory. An intercsting review is by Roginski on "Development of the Theory of Relay Circuits in the U.S.S.R." The theory of relay circuits was seriously begun by Shannon and Bell Laboratories; and Shestakov and the Russians in the late ' 30 s, applying a Boolean algebra to relay contact systems. It is interesting to note, philosophically, that the questions were likely first raised closer to the time that the problems of symbolic logic in modern form - about 1910 - were being developed, by outstanding European thcorists (illustratively, Russel1 and Whitehead's PRINCIPIA MATHEMATICA). Roginski quotes a 1910 passage from a review by $P$. Erenfest of a book on symbolic logic which raises the question whether there are complex communication systems in physics and engineering, for example, automatic telephone exchanges, that will require complex logical systems as a sort of algebra of distributing systems. Thus, philosophically by 1910, theoretically by 1930, and put to use in the form of automatic relay network synthesizers both by the Russians and Bell Laboratories in the late '50s - the Russians insisting on a first priority claim as of 1956 under Roginski - and a great number of theoretical papers in the late '50s traces much of the development of interest in this field.

However, one may note an opposing view by Popovich, representing a Rumanian group of workers, who does not believe that the problem of analysis of switching systems is completely solved, particularly with regard to multi-contact switching systems. Determining the operational sequence of elements in time was solved by both the Russians and Shestakov and a Rumanian scientist, Moisil. Determining such sequences for the circuits containing receiving 
elements cannot as yet be regarded as solved. Here the complexity, in contrast to autonomous circuits, is due to the non-uniqueness of transition from one state of the internal elements of a circuit into another state, which depends on the state of the receiving elements.

It is thus most interesting to find the following paper by Moisi1, "On the Synthesis of Switching Circuits: With Relays and Contact or With Electronic and Solid-State Devices". He makes the point at the outset that the initial methods of Shestakov and Shannon made use of Boolean algebra, whereas Moisil has proposed to use others in addition - for example many valued logics of Lukasiewicz and Glaois fields. Moisil references his 1959 book ALGEBRAIC THEORY OF SWITCHING CIRCUITS. The paper is too complicated to review here. Closing remarks of Shestakov really pay tribute to Moisil by first noting that although the Rumanian work is only recent - 1954 - already it is second in European size to the Russian; that in departing from Boolean algebra, they captured Russian fancy; and that they were also able to demonstrate practical switches.

(Non-Boolean switching, in recent years, has begun to capture many fancies. We, among others, consider it significant for general communications theory. We are interested in and seek and await the development of physical network theory upon which to found a communications theory. The ends are the same; the direction of approach is different. One can point enthusiastically to such switching network theories as an attempt to get at the over-a11 properties of the physical network from a logical point of view. It certainly has become the electrical and electronic engineers' problem. A likely reason that they have concentrated on logic problems is that the electrical-electronic passive and active elements are in hand and they are concerned with the structure of systems that can be built cheaply and complexly from such elements. This is in process now of changing. The general physical elements that plague the physicist on the other hand are broader and not all so certain. This is certainly the case in the biophysical system. The physicist thus expresses a natural caution in attempting to decide with what he is trying to synthesize.)

A modern Russian view of noise suppression in communications networks is contained in a paper by Venchkovski etal. A number of works (1956-1959) have shown that modern communications systems practically achieve the potential theoretical noise suppressibility in many cases. The theory is reviewed for both strong and weak signals for AM, FM, pulse frequency modulation, pulse width modulation, and pulse code modulation combinations.

Another paper, by Nemicki, stirs up but does not resolve a basic question of what constitutes a steady-state regime in non-linear systems with many degrees of freedom which may evidence, among other vague characteristics, periodic or almost periodic regimes with very long periods.

Current 'frontier' theory is contained in such efforts as Kalman's to find a foundation for optimal control systems description. Earlier descriptions by Bellman of so-called dynamic programming and this paper by Kalman have helped break out a phase-space description of dynamic systems known as state-space techniques. Another pole of theory is contained in such continued 
work as Flügge-Lotz in discontinuous control systems. The dynamics of such systems are finally becoming explored as indicated by such papers as subharmonics in relay servos, bang-bang control discussions, etc.

Passing through all four volumes indicates that there is a considerable discrepancy between the theory papers and the practical and applicational papers. It is commonly considered that theory is ahead. (However one may have considerable concern with to the real state of affairs. While the papers all have considerable complexity, there are few that really offer any kind of basic illumination to other investigators or to practitioners. Instead, what one sees is a polite acceptance by various authors of the author talking at the moment and their continued searching for a new idea. One cannot feel that this Congress really advanced any new automatic control art, although there are many hidden seeds.)

For a next illustration, the 1962 JACC (21) may be inspected.

Moore's keynote address on the frontiers of integrated automatic control systems essentially summarizes the state of development of the automatic control field, which he points out was sparked through World War II by billions of dollars spent in the defense effort. (It is thus hardly possible to regard the content of these meetings as not representative of all but a few classified ideas.) The computer (and its logical limitations) and the feedback servo network represent the main tools and ideas that have been developed to date. Its achievements are shown by defense applications in the automatic crew for vehicles - the gunner, pilot, navigator, etc.; in command, communication, and control functions; in management aids; even as a memory in proposal preparation; in automation of manufacturing and plant operation. New (technologica1) frontiers have developed for and from this in micro-miniaturization, amplification, distributed field phenomena, automatic traffic control, vehicle control, space exploration, ocean exploration, and communications. (All of these have been straightforward physical and engineering developments. To marvel at their 'automatic' development is no great thought.) other breakthroughs are in lasers, in business machine applications, in the concept of adaptive control, in bionics, in automatic reconstruction, in automatic language translation, in the possibility of the automatic oraclc, in automatic information retrieval, and automatic medical diagnosis and treatment. (It is worthwhile to comment on these. The business machines applications were routine, and of technological significance. Whatever progress has been made in the other fields, except for the maser and laser, have all been of an engineering nature. The philosophic and 'theoretical' formulations of the problem have been grand, even grandiose, but the actual achievements are based on good practical modern engineering. Language translation is an excellent point in which the linguist, logician, mathematician, scientist, all construct considerable verbal theory, but the IBM engineer builds the working machine more nearly by common sense. Thus, as usual engineering progress is considerable, based on attention to many details; but scientific progress is based, in the main, on major simpe ideas. These two facets cannot be confused.)

Ashby's invited address deals with what integrated automatic control can learn from the brain. starting from the premise that brains and computers are 
essentially the same device, he proposes to sketch their relationship. The first translational step was Cannon's formulation of homeostasis, equivalent to feedback servos. (This is not true. Cannon proposed regulators.) Game playing is the next important step, and game playing constructability in computers has demonstrated that intelligence is achievable. How may this be done? By the engineer building complex enough regulators that are effective. Some essentials in the structure are an invariance of achievement in spite of disturbance, which is homologous to removing noise from a corrupted message. However, the amount of information that has to be processed is astronomical. The key problem is thus to find ways to do the job with tremendously greater efficiency. (The essence of the matter is not the philosophic question, but the levels of 'practical' questions. What physical logic and what engineering logic shall one put in? The philosophic generalization is worth the following dialogue. "Man is a computer". "True". "He can be simulated". "True. But how?".)

The paper of Chestnut etal on automatic optimizing is a good example of current ideas, pursuing Box's work of a number of years ago, of 'practical' strategies to be used for the control of poorly defined processes.

In this meeting the so-called state-space techniques come into popular prominence in the papers of Zadeh, Desoer, Bertram, Kazda, Schmidt, and Higgins. As Zadeh presents it, its key is a phase-space - common in many branches of physics involving many degrees of freedom - of time dependent inputs, the input function space; a set of time dependent outputs; and a set of state variables which characterize the system at a starting time $t_{0}$. If the inputs are known over an interval $t_{0}$ to $t_{1}$, then the output set depends only on the initial state set and the input set. The two state equations express the idea that the new state of the system depends on the initial state and on the time course of inputs, and similarly for the output. On these ideas, further technique is built. (These ideas, stemming here from Bellman's ideas of dynamic programming, and Kalman, are not physical dynamic models but concepts of mathematical continuation across complex systems. As such, they can perhaps lead to useful techniques, although the burden is on the originators to show this; but, they do not solve physical problems. This is not a criticism but a statement to locate their position in a hierarchy of science.)

Fl'ugge-Lotz' paper on optimal control with minimization criteria is an important contribution.

There is an interesting paper by Rosenblatt who attempts to get at the problem of the mind, the same problem that will be discussed later in this report. The question he raises is how to get at a brain model by physical science without straying too far from reality. Historically, a path was first taken by Rashevsky to analytically model the neuron; then using the near- empirical idea of a continuously circulating impulse in a loop of neurons. This has appeared to be too complicated for the actual mind structure. Then, McCulloch and Pitts proposed the on-off neuron switch, leading to Boolean function computer-like networks. This approach has been self-defeating. There does not seem to be any network specificity. Instead, one is more tempted to advocate statistical models for neural networks. The 'perceptron' model is 
discussed, which in most elementary model form is made up of a response unit, with connections from all pertinent amplifier units; there are connections from stimulus units to amplifier units; and series coupled from amplifier units to the response unit. The amplifier and response units are threshhold devices, and the response unit is binary, +1 , if the inputs sum is positive; -1 if the input sum is negative. (Perceptron development has had interesting and useful engineering characteristics, but it has not solved the problem of the mind.)

There were also a number of papers on learning.

A final item reviewed was the 1963 JACC (22).

The paper by Chestnut eta1, a second part continuation, is a good example of what modern complex control theory is about, and what it is to be used for. (At the same time, it illustrates the real limitations of what can be done in today's control theory.)

There are a considerable number of papers on bang-bang optimalization.

A peek at the future is contained in a very interesting paper by Holzmann on Pulsed Phase-Lock Loops. This is another excellent example of what the future problems in non-linear mechanics may fruitfully resemble. The paper is an example of control in the communications field, applying nonlinear feedback control ideas to analyzing a phase-controlled oscillator operating in a so-called phase-lock loop. There are time-varying, non-linear systems designed to establish and maintain zero frequency difference between an input carrier and a local oscillator. Feedback constrains the oscillator to follow frequency variations of the input carrier. Such pulsed loops are distinguished by the fact that the input is a pulsed intermittent sinusoid. Since the input is not always present, the pulsed loop sees no error between pulses; but by design, it can remember phase information from previous pulses and thus maintain synchronism. Thus, it can be operated either as pulse-to-pulse synchronization, or pulse-averaging.

A paper like Parks' on "A New Proof of ithe Hurwitz Stability Criterion by the Second Method of Liapunov...." furnishes a Liapunov-Routh-Hurwitz stability criteria link and will help simplify these concepts in automatic control theory for many, and brings the matters closer to the mathematics of differential equations.

A paper by Lee on "Optimization by Pontryagin's Maximum Principle on the Analogue Computer" is illustrative of the continued interest and search for methods of optimizing process paths. Comparisons are afforded between the use of Bellman's dynamic programming as compared with the maximum principle computed on an analogue computer for the desired problems, for which the classical calculus at variations is not adequate.

There are quite a few papers on stable, non-linear control system design via Liapounov's second methods, many inspired by Kalman and Bertram's 1960 paper. The field of application still remains more theoretical than practical. 
Thus, without further editorializing, it would still appear reasonable that Chestnut's November 1963 summary represents a clear statement of the state of the art.

\section{Toward a New Control Theory}

As an apt introduction, the reader is referred to a similar, but lesser argument carried on in (9). It was argued with considerable detail that the common electronic sources, at least in the past, have not really stated valid conditions for oscillating networks in their usually linearized network descriptions; that this weakness shows itself most clearly in their need to generally characterize oscillators in two categories, negative resistance networks (which, while a suitable mathematical fiction, is physically 'impossible'; more recently books discuss bistable network ideas) or feedback networks; that these books lacked a general non-linear view of oscillator design; and that at least, for quality oscillators, a primary non-linear theory has existed for quite a long time.

Now, it is necessary to come to a similar problem for control purposes. Intuitively, one must recognize that control is something diffcrent from general network dynamics. Practitioners in the field are certain of this. However, a control of systems has never really been defined in a general dynamic sense.

It is clear that the designer is concerned with system response which remains bounded, i.e. which does not depart toward infinity. Elementary theorems, of course, identify such behavior with simple periodic functions. It has become conventional in this field to then identify the simple periodic function response with linear differential equations with constant coefficients. However, this chain of logic is simply not correct. Its worst sin is that it identifies cyclicality as a solution of an artificial complementary equation, instead of an association with singular states of motion of the equation system. This represents rather serious distortion of the stability criteria that led to the oscillation in the first place. Illustrated in physical terms, as a simple physical argument, a prototype linear oscillator arises from

$$
\begin{gathered}
\frac{d^{2} x}{d t^{2}}+\omega_{\circ}{ }^{2} x=0 \\
x=A \sin \omega_{0} t+B \cos \omega_{0} t
\end{gathered}
$$

However, in any real physical world the laws of thermodynamics assure that there will be damping 


$$
\begin{gathered}
\frac{d^{2} x}{2}+2 c \frac{d x}{d t}+W_{0}^{2} x=0 \\
x=e^{-c t[A \sin q t+B \cos q t]} \\
q=\sqrt{\omega_{0}^{2}-c^{2}}
\end{gathered}
$$

and the system will ultimately come to rest. (See,DenHartog (23)). Yet, the systems under discussion are constantly oscillatory - whether there is input or not. Thus, this type of linear theory with constant coefficients is not really pertinent.

Of course, the true answer is that the systems operate as non-linear limit cycles, and that the cyclicality arises from energy pumped into the system, by D.C. or A.C. sources, to generate the cycle out of the non-linear instability.

A strict physical program for analyzing these systems which will not suit classical servo control purposes, is contained in the theory of small vibrations. In this 'rigorous' theory (see (3) and (11) for example), the singular states of motion of the system are investigated. (These are the static solution states that exist if all time derivatives - i. e. velocities, accelerations, etc. - are equated to zero.) The motion in a small region around these singular states is then explored, by linear means. This is not the same, and does not involve the same stability considerations as attempting to linearize around an operating point. Necessary conditions for stability (i. e. the Routh or Hurwitz criteria) can be derived from these conditions.

After considering the possibility of stable operation in the vicinity of such a singularity (stability in the Liapounov sense), namely if the system is linearly unstable, then one explores the surrounding phase-space for stable non-linear limit cycles (i.e. closed trajectories), or unstable open trajectories which depart to infinity.

Thus the question is what constitutes the dynamics of a control system? The following key ideas are newly derived:

1. A control system intends that one or more control variables (i.e. degrees of freedom or generalized displacements), or derivatives of such control variables shall vary in time only in accordance with some reference variable. (This is not new; this is simply an abstraction of the key thought in every control designer's mind.)

Typically, the requirements of variation might be

a) To hold a control variable magnitude constant (a positional servo)

b) To hold a control variable time derivative constant (a velocity servo)

c) To make a control variable vary in accordance with some associated physical law (e.g. if $r$ changes, make $c$ change such that $c(t)=f(r))$ 
d) Make the control variable vary according to a course in time of some other system (e. g. respond in sequence time to the dynamic changing characteristic of some other system).

In permitting this item within the scope, we are beginning to destroy the general validity of the distinctions between regulation, particularly dynamic regulation, and control that were advanced in (1). Yet, the issues at stake here are quite complex, and nearly philosophic. (It is possible that two different meanings of time are implied, such as tabsolute time, i.e. the earth rotating. A system metering time in accordance with earth rotation may still be left a controller, while a system in isolated space-time metering out time or frequency might still be left as a frequency regulator.) It is possible that a most general relativistic mechanics would be required to resolve the problem. Yet, in all candor, we must confess to a mixed-up point of view here. However, the mix-up only arises at this time in searching for a new general theory of control. As far as (1) could go, it was consistent there. Now it is not so consistent.

2. If the physical laws of nature permitted a system to vary in accordance with the desired variable, then the answer exists and a control system is not needed. (This is partly new, as the following discussion will indicate.)

This almost trivial thought contains some decisive elements for control theory, as a number of illustrations, both trivial and profound will indicate. It is obvious to all but the very naive that a control system, say to hold an output variable constant, cannot be constructed by simply pinning the output variable. Yet, it has certainly defied an explanation other than that such a pinned no-degree of freedom system is just not a control system. The argument of what is a control system always becomes circular. Now it is simply answered with an offhand explanation that is no more or less trivial than the question. Nature permits the system to be simply pinned, and therefore this is not a control system.

If the response desired is

$$
m \ddot{x}+c \ddot{x}+k x=y
$$

between output $x$ and input $y$, then this is not a control system but a simple nearly achievable physical system.

This simple-minded thought also contains the corollary that if the 'isonchronous' response

$$
m \ddot{x}+k x=0
$$

is desired, namely if a simple timing function is desired, this is nearly achievable physically, and is therefore not a control system. (It is not necessary at this time to go into all the subtlety of measurement theory as to what constitutes physical achievability. There are well known uncertainty 
principles invokable on the quantum level, on the molecular level, and on the 'practical' level so that no absolutes in accuracy are intended, but accuracies in space and time in accordance with specific investigation needs.)

Thus, control systems must start from systems in which physical dynamics do not permit the system to behave as is desired.

3. The physical problem of control theory, an apparently null problem, is to physically modify the given controlled system response so that it will fit the desired reference input response. (This, we submit, is an original thought, and perhaps new, as far as we know at present.)

This might leave the reader confused, or breathless, or ready to dismiss the whole affair as being trivial. However, it is not. It is simply the difficulty of achieving the desired modification that creates such a high ordered problem for the designer.

The reader must understand that by the term 'given system' there are implied constraints that are not obvious. The usual physical constraint that exists is that the given system has mass (it is a real airplane, or missile, or ball, or arm, or blood system, or torso, or social system of 180,000,000 people that is involved), and thus physical laws of inertia - usually undesired are involved.

If Newton's law says

$$
\mathrm{m} \ddot{\mathrm{x}}=\ldots
$$

it is not possible by physical means to whip into the system a negative mass

$$
(m-m) \ddot{x}=\ldots
$$

that will knork out the second derivative term. Thus, it must be lived with and designed for. If the first law of thermodynamics shows minimally

$$
m c_{v} \frac{d T}{d t}=\ldots
$$

that thermal mass will store energy by virtue of a real specific heat of the mass, thermal time delays similarly cannot be brushed away.

The references to Chestnut and Young before were not trivial scientifically. If in a complex system, a control function requires coordination of a large number of elements, the 'economics' of the control solution is a fundamental consideration. Nature has learned how to operate the physical world with 'cheap' particles, the biological system with 'cheap' cells. A complex man-made control system is still quite a monstrosity (it is to the credit of communications companies, and computer technology that such problems have progressed as far as they have. No insult of man's endeavor is intended, just a statement of the level of progress.) Thus, repetitiousness of elements, and the need for designing with simple - not perfect - response characteristics (such as two state switch elements) for quality response is an intrinsic, not 
incidental, portion of the theory of automatic control of complex processes.

Besides the constraints imposed by the 'given system', there are the constraints on the 'modifying control system'. A primary one is power. The designer generally assigns an external power supply of sufficient level to approach some level of acceptable response.. (It generally boils down to the fact that the desired system response cannot be fitted identically, but only approximately. Thus, a level of error is assigned, and a sufficient actuating level of power is assigned.)

Other constraints that exist may be the speed of response with which errors between the desired and the response functions are permitted to build up and, perhaps not so obvious but clear to most thinkers about control systems, a philosophy of design. This latter point is always a major issue, in whether one is talking about (a) linear servo theory, (b) bang-bang control, (c) adaptive control, (d) other non-linear control, (e) computer control, (f) higher ordered 'cybernetic' machines, or (one that we enjoy completing the list with), (g) eighteen-year-old stenographers.

So far this program of ideas has not laid out the design problem. While some mathematical statement of functional identity might be named for the fitting of control response, it is likely more useful to characterize the problem in a topological non-linear sense. This will be attempted for a one-degreeof-freedom system. It will not be really possible to build up a complete theory here, but just to sketch out its beginning. (For the sake of a record of references for the reader, he is referred to the work of S. Lees (24) which shows remarkable clarity of design philosophy. What is particularly admirable is the wisdom of design direction achieved with obviously no general nonlinear guiding theory, but with considerable physical intuition.)

4. Consider the following phase plane analysis of a control problem, say here a positional servo.

A one-dimensional phase plane is the following

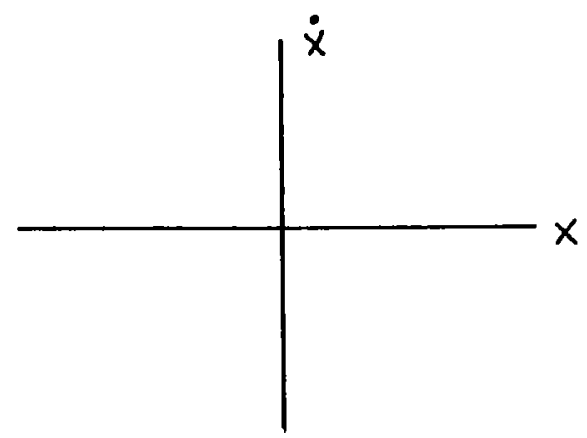


More cumbersome (as Minorsky (15) states on p. 8) one may visualize a phase space

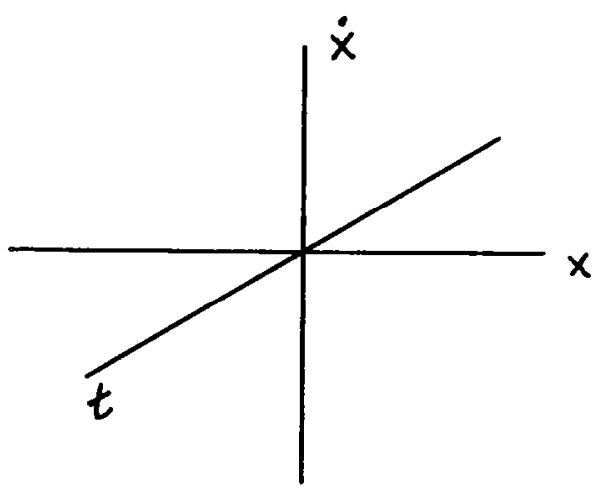

For simplicity, consider a positional servo that is required to hold a constant displacement in the face of disturbances.

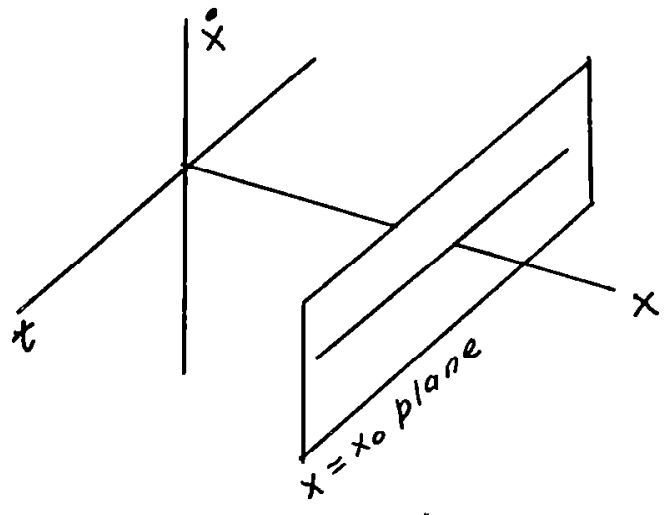

$x=\int_{0}^{t} \dot{x}(t) d t$

As a mathematical problem the issue might be viewed simply as developing a correction system that makes the velocity integral average to zero. However, this does not recognize the physical constraints which generally involves an inertial term and some restoring term. Thus, it is likely most general to regard the design problem as involving design inside a rectangle in the simple phase plane.

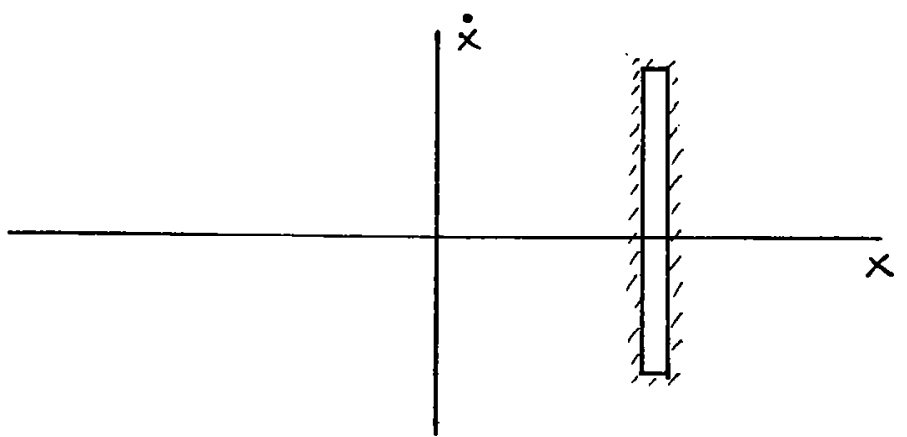


It is possible that the rectangular nature of this solution domain (for a positional servo) has led to the idea of solution by linear servo ideas, for in a linear case, the approach to the singularity would be by

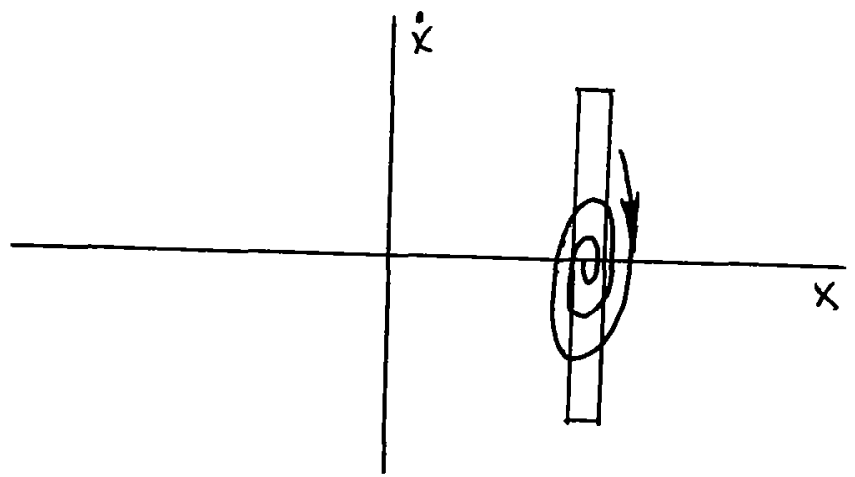

which would approach stability in the Liapounovsense. However, this is not the only solution. Another type of solution, for a given positional error however small - may be a limit cycle nested with the rectangle, such as

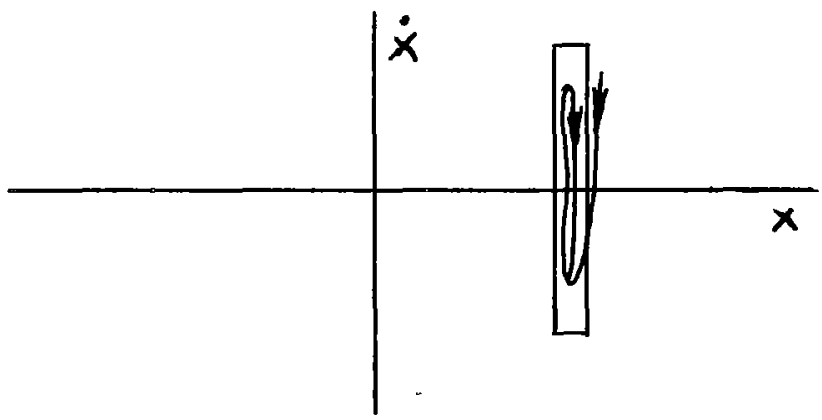

Thus, our ideas first considered in 1953 were not completely correct. The solution to each control problem is not a unique non-linear limit cycle, but a non-denumerable family of limit cycles and stable linear trajectories that fall within the control region by approaching the singularity as a focal point.

If more generally, the desired reference input response is positionally variant - for demonstration purposes, say, very slowly varying, so that the input response on $1 \mathrm{y}$ has small displacement derivatives

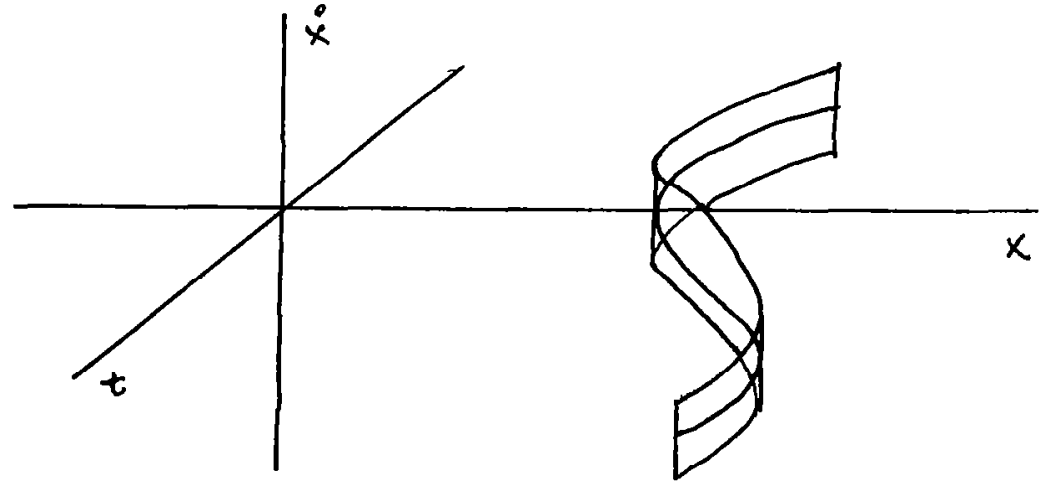


Then the problem requires a horizontaliy thin limit cycle or spiral that can follow a shifting singularity.

We are frankly not prepared to solve the entire design problem raised by even this primitive beginning. It will require intensive study design example by design example.

Since the solution optimalization may generally be involved for both external disturbances (which pluck $x$ and $x$ from its 'equilibrium', whether linear or non-linear) as well as changes in the reference function or functiona1, there is a more general requirement than just the phase space displacement $x-x_{0}$ for a constant $t$. A certain error volume drawn around the desired path, may show more optimal solutions by means of non-rectangular phase plane solutions, i.e. they may be curved, involving warped limit cycles as shown, etc.

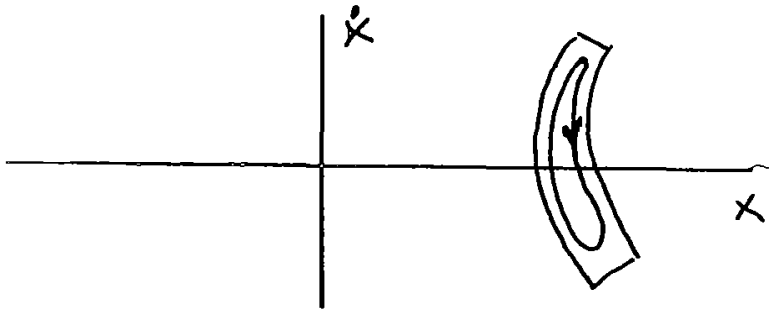

Thus, considerable complexity - including discontinuous functional properties - can exist.

Furthcrmore, one may add morc complex control specification rather than simple positional control, typically some simple combinations that could be imagined are to make:

$$
\begin{aligned}
& c(t) \text { respond to reference } r(t) \text { by } \\
& c(t)=A \frac{d r}{d t}+B r \\
& c(t)=A\left|\frac{d r}{d t}\right|+B r
\end{aligned}
$$

= absolute value

$$
\begin{aligned}
& \frac{d c}{d t}+A c=B r \\
& c=A r \frac{d r}{d t}
\end{aligned}
$$

etc.

The point is that there is no 1imit to the complexity of the control response that might be invoked. All of this will have very specific connotation in biological systems. 
The final complexity that will be mentioned is, of course, the one that stems from control of more than one variable in a complex system. When these are non-interacting, they can be treated independently. However, when there is any appreciable coupling, which is always the case in so-called feedback systems, then interaction can make the over-all problem very complex. In principle this should be handled in a hyperphase space. In practice, it will be handled by the art of the investigator.

Succeeding in finding this vantage point from which to view general control theory has not simplified the problem; it has only given the designer a more general view of the problem, and a door opener to more general methodology in non-linear mechanics. Acknowledgement must be made to the great contributions of Poincare and Liapunov and to Minorsky, who have laid the background in this field. The reader is thus urged to live with (11) and (15) as a beginning toward understanding of non-linear mechanics.

For those who might regard these ideas as overly complex, we can only refer them to the papers of Bellman and Kalman, or the large amounts of Federal research funds that have been spent in attempts at the second method of Liapunov. For those who regard these ideas as overly simple, they can review the JACC and IFAC Conferences in great detail themselves to seek over-all nonlinear mechanical or topological views of the entire control problem.

As a brief introduction to control in biological systems, the previous general introductory theory to automatic control was based on having first caught a glimpse of a second corollary but very basic idea which will now be discussed. (In doing so, these two ideas represent a flowering of the primary hope contained in the so-called program of bionics. We must confess to having had a serious distaste and doubts in the idea that either the physical scientist would learn about control from the biological system, or that the biologist would learn about control from the physical scientist. Yet in this study, the idea appears to have worked.)

The biological system consists essentially and almost completely of a system of limit cycle chemical oscillators whose nearly constant but shifting chemical-electrical levels represent singular states of these oscillators. These levels, which result from the limit cycle operating domains, represent the general homeostasis of the biological system. (It is likely Van der Pol to whom major credit is due for bringing the significance of the limit cycles, in general in biological systems, to scientific attention.)

The general problem in biology will be to expose the foundations for and the sources of these limit cycles. Parts of this problem, in particular and in general, will receive exposition in the remainder of this paper. 


\section{DYNAMICS OF THE THERMOREGULATION SYSTEM}

\section{A. Theoretica1 Dialogue Continued}

A test of the validity of the ideas of general control theory, and of the general modelling of the biological system in particular, arises aptly in the first system topic under discussion in this study, the temperature regulation system. In (2) the argument was pursued at length regarding a rudimentary model of this system, and connected with Benzinger's excellent data. Now it will take what may appear to be a widely different theoretical turn. In a number of papers (4) the sustained dynamics of the ventilation and metabolic processes in the human were experimentally shown.

Following the lead of Tustin, this work established the nature of biological system dynamics in a manner that would not be strange to the fields of automatic control or non-linear mechanics. Following logically after the better known system dynamics in the cardiovascular system (the heart beat) and in the nervous system (brain waves, action potentials, etc.), these more recent demonstrations of a sustained power cycle, as evidence of an overall coordinated engine cycle, was a very significant addition to part of the fundamental operation of the biological system.

A number of other investigators have very recently seen fit to provide such data with a different interpretation. In 1961, Goodman (8) duplicated results in (4), first starting by computer reanalysis of the earlier data, and then by taking ventilation data on a considerable number of other human subjects. While moderate differences between the two investigations were found, it was clear that both experiments revealed a similar spectrum of intrinsic limit cycle frequencies in the ventilation rate. Goodman's data were more elaborate, and he was able to cast some light on the question of whether these frequencies were stationary constants for the system, by showing that they tended to wobble over the time domain.

Following,this work, a student of Goodman's, Clegg, has done a modelling study in 1963, of the respiratory dynamics. This has recently been released as a Case Institute study (25). Its timeliness, following the work of Gray, Grodins, and Defares, make it a suitable subject for analysis.

Although clegg attempts to model the ventilation dynamics exhibited in (4), that work is inexplicably not referenced. Another line of thinking, one that regards the ventilation dynamics to result from $\mathrm{O}_{2}-\mathrm{CO}_{2}-\mathrm{pH}$ sensing and flow transit time lags is presented.

The difference in the two views is so important that the Gray-Grodinsclegg modelling will be presented here in considerable detail.

One may regard that Gray (26) began a realistic modelling of the chemical factors involved in ventilation. From empirical data, he derived the result

$$
Q=Q_{0}\left[0.22 \mathrm{pH}+0.262 \mathrm{pCO}_{2}+\frac{105}{10^{.063} \mathrm{pO}_{2}}-18\right]
$$




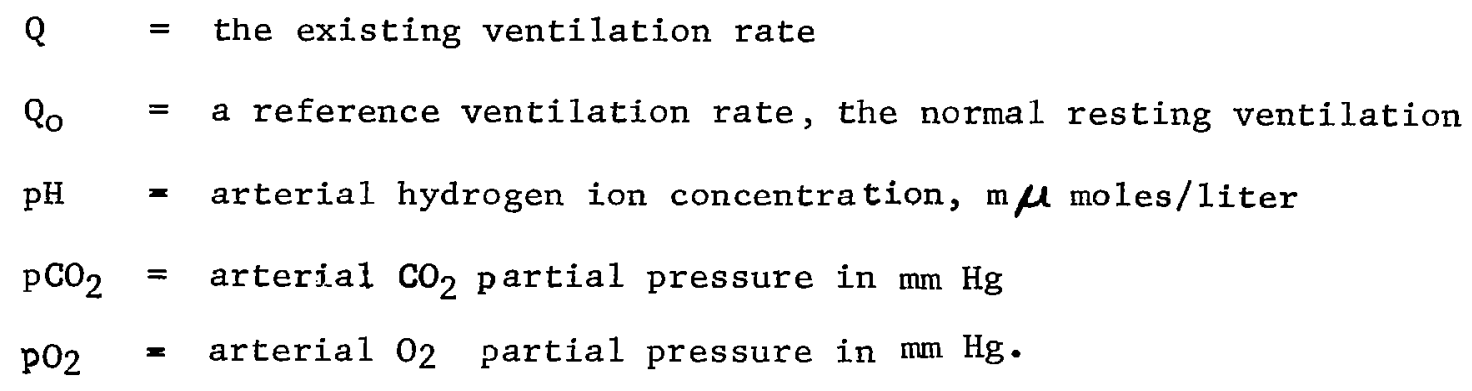

The above relation, erroneously regarded by many as an expression of the chemical regulation of the pulmonary ventilation, exhibits the factors that influence ventilation rate. (They do not physically model them. Thus it is not clear whether the above equation represents a mass balance, a chemical balance, or a force balance. This is meant to imply nothing derogatory about the excellence of Gray's work).

It is likely Grodins work (27), attempting to pursue Gray's beginning toward a model of the chemical regulation of respiration during exercise by $\mathrm{CO}_{2}$, that starts a modern dynamic systems view of how it might be possible for a physiological variable like ventilation to achieve a "feedback regulation't in the face of disturbances such as exercise. (Grodins' work does represent a very good first effort to make use of control theory concepts. In particular it is quite pleasing to find a book like Trimmer's RESPONSE OF PHYSICAL SYSTEMS referenced in the 1954 paper.)

However, it is clear that Grodins was concerned in the main with the transient - say step function - response of the human to $\mathrm{CO}_{2}$ levels in the blood. In his 1950 paper, he is concerned with how the regulation of ventilation (in this context the term regulation is used with precision) takes place in accordance with oxygen consumption, i.e. according to Gray, ventilation and oxygen consumption and metabolism are directly proportional. As Grodins puts it quite well, an adequate theory of respiratory 'regulation' (he said control), during disturbances of exercise, must account for this proportionality. Grodins asks, very validly, whether this regulation can be accounted for by the body fluid chemical agents of $\mathrm{pCO}_{2}, \mathrm{pH}$, and $\mathrm{pO}_{2}$.

In his 1954 paper, with considerably more engineering sophistication, the question is raised as to whether blood ventilation, blood and tissue $\mathrm{CO}_{2}$ level, ventilation $\mathrm{CO}_{2}$ level are related in an automatic control sense in which ventilation is the reference input variable ("controlling quantity"); $\mathrm{CO}_{2}$ body concentration (at some site) is the controlled response variable; and $\mathrm{CO}_{2}$ concentration in the inspired gas is a disturbance quantity.

Literally, if these two papers were involved with what they purport to be involved, then exercise, which would demand some increased but undetermined level of metabolism, would involve the oxidation of a certain rate of foodstuff with some 'specific dynamic action' (i.e. an appropriate R.Q.), which would involve a particular correlated nearly-regulated ventilation rate, which by Grodins 1954 paper, would involve an automatic control response to maintain $\mathrm{CO}_{2}$ concentration in the body tissue against even 
disturbances of $\mathrm{CO}_{2}$ level in the inspired breath. Thus both exercise and $\mathrm{CO}_{2}$ inspiration level are disturbance variables, whether regulation or control is involved (i.e. whether one is talking about static steady state results, or slowly varying level adjustments to exercise power demand or $\mathrm{CO}_{2}$ inspired breath variation). While this would reveal regulation and control dynamics of the entire system if appropriately measured, this does not imply that the dynamics of the power system is being explored or that even a power system dynamics is implied, only that regulation or control follower-type dynamics may exist in ventilation. However study of the 1954 paper reveals that only the response to quasistatic regulation upsets is investigated, and not automatic control functions, and only transient dynamic regulation upsets (i. e. step functions) in $\mathrm{CO}_{2}$ input disturbances is investigated.

What does Grodins 1954 paper demonstrate? He shows -

1. Following a step function of $\mathrm{CO}_{2}$ level in inspired air. the $\mathrm{CO}_{2}$ in a cat's tissue changes in level according to an elementary linear first order reaction with an experimental time constant of the order of 28 minutes (his Figure 5). He assumes -

2. Pulmonary ventilation is determined by "body $\mathrm{CO}_{2}$ concentration," although it may be localized at a site, under both static and dynamic conditions, and the ratio is very nearly linear, $i$. $\boldsymbol{e}$.

$$
\mathrm{Q}=\mathrm{A} \mathrm{V}_{\mathrm{CO}_{2}}+\mathrm{B}
$$

$\mathrm{Q}=$ ventilation flow rate (minute volume, e.g. 1pm)

$\mathrm{V}_{\mathrm{CO}_{2}}=\begin{aligned} & \text { volume of } \\ & \text { pressure) }\end{aligned} \mathrm{CO}_{2}$ in the tissue (e.g. cc's at normal temperature and

$A$ and $B=$ constants

(This is purely an empirical assumption, not incompatible with Gray's ideas, and not incompatible with physiological ideas of a respiratory center. The specific linearity type of assumption is more nearly a convention of linearizing than a statement of essential need for his model. However as Grodins clearly states, it is not so easy or possible to test whether such convention relations hold both statically and dynamically. However Grodins offers work of Hessner in which ventilation changes parallel changes in venous $\mathrm{pH}$, not artcrial $\mathrm{pH}$, as evidence of the primacy of $\mathrm{CO}_{2}$ tissue concentration, and some work of Lambertson).

He assumes -

3. Five kinematic relations, of which two are mass rate balances of $\mathrm{CO}_{2}$ in the gas lung space "reservoir", and in the fluid tissue "reservoir" respectively; two are partial concentration mass balances (by partial pressure) of $\mathrm{CO}_{2}$ to total gas in the gas space, and of $\mathrm{CO}_{2}$ to total fluid in the fluid circulating through the tissue; and the fifth is the assumption of a functional relation between the rate of removal of $\mathrm{CO}_{2}$ by the arterial blood flow and the partial pressure of $\mathrm{CO}_{2}$ in the lung 
storage space. The six variables $-\mathrm{CO}_{2}$ flow rates in (1) expired gas; (2) arterial blood; (3) venous blood; (4) tissue fluid reservoir; (5) lung gas reservoir; (6) pulmonary ventilation - lead to two capacitance (i.e. R-C coupled single capacitance circuits) equations of the form

$$
A(Q, \dot{Q}) \ddot{x}=B(Q, \dot{Q}) \dot{x}=C(Q, \dot{Q}) x=f(Q, \dot{Q})
$$

where $\mathrm{x}$ may be the volume of $\mathrm{CO}_{2}$ in tissue, or the volume of $\mathrm{CO}_{2}$ in the lung space;

4. Eliminating the ventilation rate, in turn a second order non-linear differential equation can be set up for

$$
\begin{aligned}
& \text { volume of } \mathrm{CO}_{2} \text { in tissue } \\
& \text { ventilation rate } \\
& \text { alveolar } \mathrm{CO}_{2} \text { partial pressure }
\end{aligned}
$$

5. Thie solutions are examined under steady state conditions, and then as a transisnt response to step functions of input $\mathrm{CO}_{2}$ partial pressure levels.

Now while Grodins, et a1, regard their equations as controller equations (i.e. closed loop feedback control) they are not. The represent the dynamics of a process, or system; in particular of a regulated process. What they are demonstrating is some of the transient time delays in the response to a regulated steady state to take place. (This explains a difficulty in understanding many billogical investigators, who continue to raise questions on what the meaning of a set point is in a system that doesn' $t$ seem to have a sharp set point. For example, this argument seemed to exist at the November 1963 16th Annual Conference on Engineering in Medicine and Biology in the tutorial sessions of Jones of Northwestern on physiological contro1 systems, and Grodins in an open forum session on control. As a reviewer in Science, Ju1y 26, 1963 of Grodins CONTROL THEORY AND BIOLOGICAL SYSTEMS stated "more often than not the biologist is still unaware of the rigorous foundation, or the complex ramifications of modern control theory."

Anticipating such difficulties in (1), an attempt was made to distinguish between regulation and control, indicating that control implies no steady state errors. Thus any system which has a complex of responses in the steady state is not a control system. The very lack of a sharply defined control point in steady state is what generally assures that one is not dealing with a controlled system. Thus it is most pointed to examine Grodins steady states to determine what regulation he is discussing).

Grodins' five kinematic relations lead to the following two 'potential equations, i.e. there are two capacitances that are 'charged to their equilibrium values. 
The first is an equilibrium in the lung space:

partial pressure of $\mathrm{CO}_{2}$ in lungs

= partial pressure of $\mathrm{CO}_{2}$ in inspired air

+ total (barom.) pressure $\times\left(\frac{\text { rate ot } \mathrm{CO}_{2} \text { produced in tissue }}{\text { rate of gas ventilation }}\right)$

The second is an equilibrium in the tissue volume:

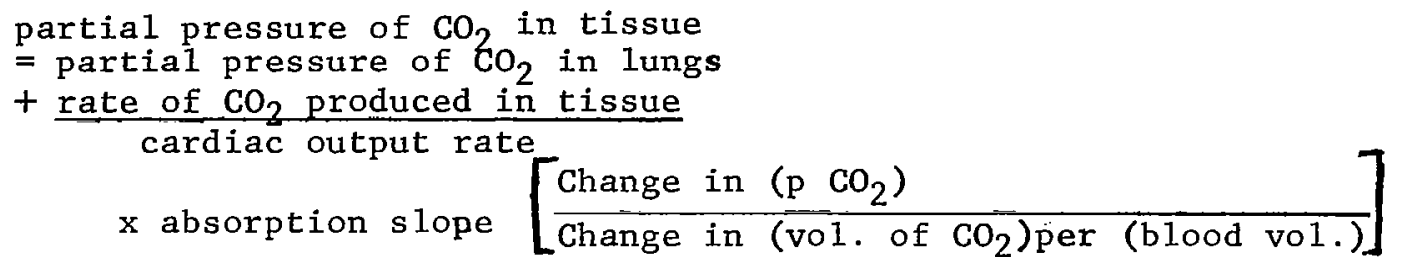

Then the third result, stated before, is the regulation characteristic of some sort of respiratory center

$$
\mathrm{Q}=\mathrm{A} \mathrm{V} \mathrm{CO}_{2}+\mathrm{B}
$$

It is these three relations, the first a pure mass balance of $\mathrm{CO}_{2}$ in and out of the lung space, the second a mass balance statement that the 2 system produces $\mathrm{CO}_{2}$ internally and has enough capacitance to charge itself up further with $\mathrm{CO}_{2}$, and the third an empirical and elementary description of ventilation regulation, that make up the foundation for Grodins' paper. This does not even really describe any regulator, only the consequence of assuming the simplest possible regulating assumption.

However, even these very simple relations are likely surprising in showing a variety of interrelations among quantities like ventilation, $\mathrm{CO}_{2}$ level in inspired air, cardiac output rate, and $\mathrm{CO}_{2}$ level in tissue.

Beyond this, Grodins real contribution (since it is more reasonable to give the work of Gray credit for bringing out the ventilation relations) was that he established models for the transient responses among the ventilation parameters, and in particular checked two transients for steps in $\mathrm{CO}_{2}$ level input in the inspired air.

One precursor to the present argument exists in Grodins paper. "Finally, we wish to describe another observation which is not accounted for by the theory in its present form. It is concerned with the on transient for ventilation and explains, we believe, why some investigators (Padget, Schaeffer) appear to have reached steady states in much shorter times than others (Grodins, Nielsen). Although not so obvious in the averaged results, all of our six subjects individually showed a high-frequency component superimposed upon the gradual rise in ventilation. This is shown in Figure 30 where minute by minute values of ventilation are plotted for a single subject. The same type of cycling evident in this graph was also present in cach of our other five subjects, and can also be seen in Schaeffer's curves." 
However the significance of these high frequency components received no further notice or interpretation from either Schaeffer or Grodins. Nor has notice been taken of their existence and possible connection with heat production by Gray, Grodins, Defares, Horgan and Lange, and Clegg. It remained for (4) to exhibit them experimentally and to attempt to bring these into focus.

In (4) an engine cycle was sought in the human to provide two things the power to be available for muscular work on demand; and the heat for temperature regulation of the deep body. From physical reasons it was argued that the engine cycle had to exist at a time domain less than three hours, but greater than 4 seconds. Exploring the human temperature changes, few seconds at a time, a near $11 / 2$ - 2 minute cycle was found. Then by exploring breath by breath, corollary ventilation and metabolic cycles were found. These were at the levels of 2 minutes, 7 minutes, 35 minutes, and $31 / 2$ hours. It was proposed that the fastest cycle represented the fundamental power cycle. With no great physiological foundation, but with a general physical argument, the nominal sequence of events were visualized as follows: The neuromuscle cycle must be of high frequency both in neural actuation and in heat release (based on the work of Hill for the latter, and as discussed in (1) on the work of Adrian and others for the former). Nominally this has appeared to fall in the vicinity of ten cps. For a coordinated muscle discharge from all over the body to take place near a 100 seconds value, some large time delays are required.

The blood circulation time, in which fatigue and other chemical signals might get around with slower than neural time delays, is of the order of ten seconds, and thus some slower process is needed. The problem is to 'invent' a process slower than transit time, almost of a diffusive nature, that involves the blood.

The concept was developed that the ingoing and outcoming blood are practically identical, except for minor differences. Thus one might view the problems as a two fluid problem, where by virtue of a small difference of concentration in and out, there would be a slow build up of a net difference of this small difference component. However, such a slowed-down concentration change is the equivalent of a diffusion rate and a diffusion constant. If one adds to this a threshhold excitation level, and neural coupling, a model is conceivable in which the nature of the inward 'diffusing' signal concentration is regarded, plausibly, as hormonal.

It is possible at this last point that we may have slipped. We purposely disregarded the possibility that the signal was an $\mathrm{O}_{2}-\mathrm{CO}_{2}$ difference in the blood carrier, which could fit the general kinematic prescription, because the gas exchange processes were considered to be too fast. However, here Grodins' work creates a second possible path of explanation.

Even though his work did not deal with the high frequency (here regarded as engine dynamics of the system), Grodins did introduce longer time constants into the ventilation system and therefore into the correlated metabolism than one might associate with gas exchange or a limited oxygen storage. (One must 
recall that Gray has been responsible for firmly tying ventilation and metabolism. We have used this concept for a long time, having learned it originally from Dr. Specht, at NIH, and found it surprising that it was questioned so often. It is much clearer now that the validity of this observation should be acceptable to most ventilation physiologists,)

However, the alternate exists that the signal carried by the blood for the control of metabolism is hormonal. Thus while we have contributed a view of the high frequency engine, and have called attention to the need for the driving signal that triggers the phase of this non-linear oscillator (the engine cycle) in the blood, its source has not been proven. It may now be viewed from Grodins work, either as $\mathrm{CO}_{2}$ triggering, or by our hypothesis as hormonal triggering.

The argument thus turns now to examine the work of Clegg (25). After Goodman (8) demonstrated in more detail the validity of a ventilation spectrum, and in particular demonstrated that the spectrum is not absolutely stationary, his students then began a program designed to try to pin down the regulation. Clegg's is the first such study, directed toward modelling ventilation dynamics in the oscillatory high frequency component, in step response to ambient $\mathrm{CO}_{2}$ concentrations, and in exhibiting instabilities. (It is also pointed to note that this study, coming from an engineering group with a teaching background in control theory nowhere allude to their study as a study in control, but as a study in the dynamics of a regulated system. Thus no artificial straining for some non-existent property is sought.)

Since their model exhibits a non-linear limit cycle, it is first pointed to inquire as to what singular states of motion they have assumed (i.e. if all varying derivatives are equated to zero, what steady state, whether stable or not, do they assume).

$\mathrm{CO}_{2}$ exchange in the lung

rate of $\mathrm{CO}_{2}$ into lung $=$ rate of $\mathrm{CO}_{2}$ out of lung

= rate at which $\mathrm{CO}_{2}$ is carried from tissue by blood

+ rate at which $\mathrm{CO}_{2}$ is brought in by inspired air

$=$ (blood flow rate) $\times\left(\mathrm{CO}_{2}\right.$ solubility coefficient in blood) $\mathrm{x}\left(\mathrm{CO}_{2}\right.$ partial pressure difference - venous blood to arterial blood) $+\left(\frac{\mathrm{pCO}_{2} \text { in inspired air }}{\text { atmospheric pressure }}\right)\left(\frac{\mathrm{p} \text {. p. of dry gas }}{\text { atmospheric pressure }}\right)$ (vientilation rate)

$\mathrm{CO}_{2}$ rate from lung $=\left(\frac{\mathrm{pCO}_{2} \text { in arterial blood }}{\text { atmospheric pressure }}\right)$ (ventilation rate)

They assume,

1. $\mathrm{CO}_{2}$ mass exchange in the lung gas space

2. $\mathrm{O}_{2}$ mass exchange in the lung gas space

3. $\mathrm{CO}_{2}$ mass exchange in the tissue fluid space 
(The essential assumption here, beyond the assumption of a mass balance, is that the rate of production of $\mathrm{CO}_{2}$ in the tissue is a direct linear measure of the metabolic rate, i.e. that the chemical process in the body is an oxidation with a constant relative quotient of carbon dioxide to oxygen.)

4. $\mathrm{O}_{2}$ mass exchange in the tissue fluid space

(Corollary to the above assumption is that $\mathrm{O}_{2}$ consumption is proportional to the metabolic rate.)

Thus far, Clegg has assumed two kinematic relations similar to Grodins for a two-capacitance lung space - tissue space modelling of the $\mathrm{CO}_{2}$. However, he has added, for completeness, the two related $\mathrm{O}_{2}$ exchanges. He has partially tied these together by the assumption of the elementary chemical process invo1ving $\mathrm{O}_{2}$ consumption and $\mathrm{CO}_{2}$ production.

5. Whereas Grodins simply assumed a linear response of ventilation rate to $\mathrm{CO}_{2}$ partial pressure in the lung and arterial blood, Clegg assumes a somewhat more complex empirical relations. (One must note that this is the first and only point at which a real physiological argument is needed. The other relations all arise from very elementary physical and chemical arguments. Yet, at this critical point, all of these authors can only take recourse to some empirical observation, which already contains most of what they want to prove, namely that ventilation rate must respond to $\mathrm{CO}_{2}$ level. Much of the rest is dressing, because the basic issue is what physiologically mechanisms cause ventilation and metabolism to change in some as yet unmentioned regulated fashion.) clegg assumes, from the data of Nielson and Smith, that below a certain arterial $\mathrm{CO}_{2}$ partial pressure level (32 $\mathrm{mm} \mathrm{Hg}$ ) that the ventilation rate is only a function of arterial oxyge: partial pressure; whereas above the threshold the ventilation rate increases linearly with the $\mathrm{CO}_{2}$ partial pressure excess over $32 \mathrm{~mm} \mathrm{Hg}$. for constant arterial partial pressure levels.

clegg notes that this is a steady state relation, but will use it for the dynamic states. He also notes that this modelling is meant to represent the combined effects of 'sensors' in the aorta and in the medulla. (Herein lies Clegg's major contribution for by a series of intuitions, he proposes to represent the real system. One must note that this modelling is quite different from Grodins, who simply assumed some elementary connection of ventilation rate and $\mathrm{CO}_{2}$ partial pressure, i.e. some vague sort of respiratory center mode1. Clegg now proposes to model the respiratory center model by some audacious hunches. Our own experiences has led us to believe that one must always have recourse ultimately to the physical mechanism, and if there are those who can cast light on phenomena without that, more power to them. However, ultimately one must come back to the physical mechanism. At best, therefore, such modelling is plausibility modelling, and the work can be discussed only on that basis).

6. In simple schematic form Clegg assumed

$$
\begin{aligned}
& Q=f\left(\mathrm{pCO}_{2}\right) \\
& Q=f\left(\mathrm{pCO}_{2}\right)+\mathrm{g}\left(\mathrm{pO}_{2}\right)\left(\mathrm{pCO}_{2}-32\right) \quad \text { for } \mathrm{pCO}_{2}<32 \mathrm{~mm} \mathrm{Hg} .
\end{aligned}
$$


$Q=$ ventilation rate

$\mathrm{PCO}_{2}=$ arterial $\mathrm{CO}_{2}$ partial pressure

$\mathrm{pO}_{2}=\operatorname{arterial~} \mathrm{O}_{2}$ partial pressure

as the total empirical relation (from Nielson and Smith) He decomposes this into two parts with three parameters $a, b, c$.

$$
\begin{aligned}
& Q=a f \\
& Q=(1-a) f
\end{aligned}
$$

and $Q=a f+g\left(\mathrm{pO}_{2}, \mathrm{~b}, \mathrm{c}\right)\left(\mathrm{pCO}_{2}-32\right)$

$$
\mathrm{Q}=(1-\mathrm{a}) \mathrm{f}+\mathrm{g}\left(\mathrm{pO}_{2}, 1-\mathrm{b}, 1-\mathrm{c}\right)\left(\mathrm{pCO}_{2}-32\right)
$$

for $\mathrm{pCO}_{2}<32 \mathrm{~mm} \mathrm{Hg}$.

for $\mathrm{pCO}_{2}>32 \mathrm{~mm} \mathrm{Hg}$.

The first part, it is assumed, arises from a chemoreceptor in the arterial blood system near the aorta. The second part arises from a localized central receptor in the medulla. The first part is sensitive to both $\mathrm{O}_{2}$ and $\mathrm{CO}_{2}$ partial pressurc, while the second part is sensitive only to $\mathrm{CO}_{2}$ partial pressure. Apparently the preferred parameter choices are equivalent to ' $a$ ' approximately $80 \%$, 'b' approximately $20 \%$.

The chemoreceptor response (steady state)

$$
\begin{aligned}
& \mathrm{Q}=\mathrm{a} f\left(\mathrm{pCO}_{2}\right) \\
& \mathrm{Q}=\mathrm{a} \mathrm{f}\left(\mathrm{pCO}_{2}\right)+\left[2.1+\mathrm{g}\left(\mathrm{pO}_{2}\right)\right]\left[\mathrm{pCO}_{2}-32\right] \text { for } \mathrm{pCO}_{2} 32 \mathrm{~mm} \mathrm{Hg} .
\end{aligned}
$$

The central medullary response (steady state)

$$
\begin{array}{ll}
\mathrm{Q}=\mathrm{O} & \text { for } \mathrm{pCO}_{2}>32 \mathrm{~mm} \mathrm{Hg} . \\
\mathrm{Q}=2.1(1-\mathrm{b})\left(\mathrm{pCO}_{2}-32\right) & \text { f }
\end{array}
$$

7. The final element in the scheme is to assume that the sensor responses take place with time delays, whose form is essentially empirically guessed at. In particular the 'sensor' variables that are 'responsible' for switching on the ventilation are assumed to have simple time delay forms.

The difference between the partial pressure of gas in the lungs and the gas at the chemoreceptor is assumed to be a dead time - a transit lag for the blood.

The difference between the partial pressure of gas in the lungs and the gas at the medullary center is assumed to be a dead time - the sum of the transit lag for the blood and for cerebral-spinal-fluid to the medullary sensor; and a first order response time - a cerebral-spinal-fluid diffusion time.

Thus nothing too startling is expected in the steady state, since it was built up as carefully as it could be to include all that was needed for the mode1. 
What should appear novel is what emerges dynamically from such a model. Typical values of time constants that are assumed in the model are

transit time in blood - 15 seconds

medullary sensor diffusion time - 40 seconds

cerebral-spinal-fluid transit time - 42 seconds

The equation set contains sufficient non-linear properties to lead to a limit cycle. In one set of figures, the source of the limit cycle is the coupled in non-linear product term - of the ventilation rate and arterial $\mathrm{CO}_{2}$ partial pressure per atmospheric pressure - which determines the rate of $\mathrm{CO}_{2}$ carried from the lung space. With a medullary scnsor diffusion time of 10 seconds, this leads to a high frequency cycle of the order of 1 minute, with a ventilation rate that varies by about a factor of two (i.e. from about 0.2 to 0.1 lps). An amplitude of arterial $\mathrm{O}_{2}$ and $\mathrm{CO}_{2}$ partial pressure of about 5-10 $\mathrm{mm} \mathrm{Hg}$ is also exhibited.

In rough extent, these numbers agree with the high frequency date of (4) and (8). However, apparently Clegg was not satisfied with the need to change the medullary sensor diffusion time to 10 seconds, from a 'normal' 40 seconds. Using 'normal' time constants and changing the $a$ and $b$ levels leads to limit cycle oscillations, by virtue of the non-linear saturation curve. However, the amplitudes of oscillations are quite small. A number of other responses are shown. All in all, 1 minute cycles are demonstrated, and a variety of time constants are also demonstrated.

In assessment of this work, it is viewed as follows:

1. As an exercise, one may admire its audacity. (That it is not purely self-originating can be born out by study of such references as Dejours, Defares and Visser, and others, some of which Clegg has referenced, others of which (1) has referenced). However, as noted in (1), Defares and Visser have already pointed out that even if all known relations are not neglected for the sake of mathematical expedience, the present models are not yet isomorphic with the real system, but an abstract scheme at a first primitive state of development.

2. What is the major weakness? Clegg has assumed a constant metabolic rate, and as a consequence a constant rate of $\mathrm{CO}_{2}$ production in the tissue and a constant rate of $\mathrm{O}_{2}$ consumption in the tissue. By doing so he has thrown away the central point of his program, namely exposition of the fundamental regulatory function of human ventilation (which is obviously to carry out a gas exchange that provides sufficient $\mathrm{O}_{2}$ but does not permit too much $\mathrm{CO}_{2}$ ).

3. One might say that the measure of progress is the amount of essential physiological material that can be newly mathematically or abstractly, or better still, physically modelled in the system.

It would have been obvious to most physical observers that the physicalchemical processes that should be modelled are: conservation of mass for each of the compunents $\mathrm{O}_{2}, \mathrm{CO}_{2}$, and $\mathrm{H}_{2} \mathrm{O}$, and conscrvation of energy, involving both chemical reactivity and hydrodynamic dissipation. However, a typical reason 
for not modelling these terms would be that physiological coupling within these equations and other equations representing the physical action of physiological mechanics are not yet represented 'isomorphically.' Another reason for not modelling these equations would be that it is not a priori. clear to what extent the body can be treated as a lumped parameter field, or requires description by distributed parameters.

Nevertheless various authors mentioned have been willing to begin partial modelling of some of these equations. Fine, more power to them. From these efforts, for example, it is clear that we have neglected the storage capacitance of tissue quite serious in our thinking, and it appears that the nominal 35 minute limit cycle response found in metabolism and ventilation likely arises from this source. (In the past, while identifying this cycle as likely arising from some property associated with a large chunk of the body, we had no idea of its source. Now, at least, gas capacitance of the tissue may have a time constant of the right order of magnitude).

Clegg, at the end of a line of previous investigators, has added to Grodins work a two capacitance balance for both $\mathrm{CO}_{2}$ and $\mathrm{O}_{2}$. He already shows that non-linear instabilities can exist. However he cannot neglect the reason for the process, namely that a chemical oxidation is taking place for two purposes - the potential ability to do work on demand, and the requirement of heating the body. This requires an engine cycle which involves a cyclic time which fits the 'inertial' elements of the process, rather than any incidental time lags. (Incidental lags may make up part of the cycle time, but we do not believe that they can be the cycle determinant. This will receive further discussion). For example, in clegg's figures which illustrate the one minute limit cycle, oxygen consumption into the lung, which is represented by the product of ventilation rate and partial pressure difference of arterial and venous blood, is thus essentially proportional to the ventilation rate, in agreement with Gray's and, much later, our thesis. Yet Clegg would have the situation that oxygen consumption shows a near two to one amplitude ratio oscillation from purely gas time delays, while the causal factor, the oxidation of fuel - sugar, occurs at a constant rate. Thus the equation sets written are fundamentally incomplete. (i.e. the real issue is not the number of capacitances that are modelled, but the chain of steps that complete the entire process).

4. We still do not know the entire chain of dynamic processes that are coupled together. We can surmise, and are willing to believe with clegg and others, that one can use a two capaciatance "summing point" model (one may say that these authors have established the elementary utility of such a modelling).

Independent mass balances have to be written for $\mathrm{CO}_{2}, \mathrm{O}_{2}$ and $\mathrm{H}_{2} \mathrm{O}$ at these two capacitances. Energy balances have to be written for a number of zones. For example, we have mentioned that the body must be decomposed into a minimum of three zones - the core, the outer insulating sheath, and the muscle 'heating' layer; and it is now further reasonable that a fourth balance should really be struck for the internal lung gas space.

At this point, the difficulties arise in describing physiological 
mechanisms. For example, fuel is withdrawn from storage and converted to a circulating form (say from the liver as sugar). Does this involve time 1ags? What initiates a signal and determines the rate of conversion? Does this enter into pertinent time scale? (It obviously does). Fuel is delivered to the conversion elements. (Others might say cells generally in tissue, but we believe that there is an organized motor unit pattern). What determines the local rate, and overall rate of utilization, i.e. is there a 'feedback' signal that controls the fuel in a control linkage, or is it done by a simpler regulation of concentration? Since the conversion involves the circulating gaseous elements of $\mathrm{O}_{2}$ and $\mathrm{CO}_{2}$, then the entire question of the sensing levels for the determination of ventilation arises. (However, one must remember the Gray result and what we also have found, that both as a static description, and as a dynamic description - at least over a few breaths - the ventilation rate and metabolic rate seem to be nearly proportional). Then further with regard to the energy balance, it appears that the circulatory system is also involved in a dynamic fashion, involving physiological determinants of the relative flow division among the various blood shunting systems.

5. Thus the basic problem still remains the following: A near $1-2$ minute cycle in the body is likely a chemical thermodynamic muscle engine cycle. What determines this cycle? We have suggested that the cycle is closed by a hormonal link. Others have proposed a combustion gas products link, and have even shown a time constant in the pertinent time domain.

A near 5 - 10 minute cycle in the body likely involves the blood distributıon system divisions. What determines this cycle?

A near 20 - 40 minute cycle in the body 1ikely involves storage of combustion products in body tissue. What determines this cycle?

A near 2 - 4 hour cycle in the body likely involves the thermal resistance - capacitance of the entire body.

One general clue that may be implicit in relaxation oscillator design, and which was vaguely implied in (1) is suggested. It is possible that body cycles tend to form in the vicinity of transit times that already are near a non-linear instability. The easiest example to point to, is 1ikely the pacemaker cells in the hear. Thus we do not wish to imply any essential reservations regarding the work shown by $\mathrm{Clegg}$ and others. Gas exchange may not be the operative mechanism, and yet be in the domain of reinforcing instabilities that can lead to the actual limit cycle.

In any case, one must realize that these ideas, even if true, at present are very speculative. The subject will arise again in subsequent sections. 


\section{B. Oscillatory Temperature State of the Human}

In (2), a series of experiments were described in which temperature data and metabolic equivalent ventilation data were taken on a quiescent human for a period of about five hours. Only the mean states (of surface temperatures and ventilation) were presented. In the present report the oscillatory states are presented. It was shown in (2) that the mean state data correspond to what is known and accepted in the literature, which thus provides a necessary qualification for the dynamic data.

Fig. 1 of this report presents the dynamic skin temperature data obtained on approximately twenty body stations that are described in (2). In that report, the conditions for the experimental sequence are also described. Fig. 1a, here, presents first the raw data taken at these skin stations in four experiments at a slow scanning rate of about one reading per station per 300 500 seconds. Any higher frequency cyclic components can appear only as noise. These experiments represent the response from a supine, almost nude subject, at ambient room temperatures of $20.0^{\circ} \mathrm{C} ; 21.0^{\circ} \mathrm{C} ; 29.0^{\circ} \mathrm{C}$; and $35.0^{\circ} \mathrm{C}$.

Fig. 1b presents the data from Fig. Ia smoothed free of 'noise' (accomplished by a gentle pencil filtering and smoothing of the data). In grand average, the approximate range of the highest frequencies found are 1100 1900 seconds per cycle, centered around 1500 seconds. This may be compared with the ventilation-metabolism cycle shown in (4) which fell in about the 2000 - 2300 second range; or with the data of Goodman (8) who resolved two cycles in the 20-40 minutes range. It is believed that all these numbers are essentia1ly similar.

Fig. 1c presents the data from Fig. 1b pencil filtered for its high frequency component. What remains, in grand average, is a cycle in the range of 2500-5500 seconds per cycle, centered around 4200 seconds. This cycle range was not found previously in (4). However, it might correspond to the near 40 minutes cycle found in the data of Goodman (8).

Fig. Id presents the data from Fig. 1c pencil filtered for its high frequency. What remains, in grand average, is a cycle in the range of 6000 14,000 seconds per cycle, centered around 9500 seconds. This may be compared with the cycle in (4) which fell in about the 15,000 - 18,000 seconds range; or with the data of Goodman who found a cycle in the two-and-one-half hour range. It is believed that these numbers are essentially similar.

Fig. le presents the means of the data from Fig. Id (except in the case of some extremity temperatures which did not appear to come to physical equilibrium). These means correspond very nearly (i.e. to within not more than $0.1^{\circ} \mathrm{C}$ ) to the data presented in (2).

Fig. Ia shows that the temperature excursion range at any station was approximately $1.5^{\circ} \mathrm{C}$. This corresponds to the same range that was earlier reported by Sheard et al (28), thus attesting to the nominal accuracy of the current data. The magnitude of nominal maximum temperature derivatives is of 
the order of $\pm 4^{\circ} \mathrm{C} . / 1000$ seconds, or $14^{\circ} \mathrm{C}$. per hour! If one will accept, as discussed in (4), that unit metabolism changes are of the order of $1.5^{\circ} \mathrm{C}$. per hour, then it will be appreciated how drastic and real are the power rate changes shown by body stations or segments (i.e. any particular station, shown in an excursion around its equilibrium, can change its local rate of heat power transfer by this order of magnitude). The rate of change of temperature range corresponds in order of magnitude to the 'metabolic' and ventilation rate changes shown in (4), and corresponds to the same temperature rates found much earlier in experiments made prior to the study reported in (4).

Under such circumstances, it is very hard to accept the hypothesis that the ventilation dynamics result from non-linear instabilities associated with gas sensing and exchange that do not involve a fundamental engine cycle.

Another noteworthy item in these data is seen in the $35^{\circ} \mathrm{C}$. experiment. With what appeared to be an otherwise normal subject medically (in candor, one might describe the subject, with his concurrence, as being a somewhat nervous person), there 'suddenly' occurred 'explosions' of heat flux from his chest and back regions, driving the local temperatures up to $42^{\circ}$ and $44^{\circ} \mathrm{C}$. These data are real, and not artifacts. (A11 observers hopped right on the rapid tempe rature rises as soon as they occurred and followed the transients all the way up and down.) To what extent this is common in medical knowledge, we do not know. However, it is quite reasonable, as inspected against the background of other data, that a 'normal' cyclic state was suddenly interrupted for a moderate time $(10$ - 20 minutes $)$ by an apparently uncontrolled temperature rise and then return. The temperature rise occurred only at a high temperature, as if denoting the incipient (from a point of view of temperature) but intermittent breakdown of the temperature regulation (i.e. as if at this temperature and higher temperature there is an experience of increasing breakdown of the temperature regulating system). It is possible that one is viewing in these data the onset of heat prostration. (It is not that unique, in that a similar rise will later be shown in a test animal.)

Similarly, it is very interesting to have found temperatures (of the very extremities) which have not settled down to a cyclic equilibrium. As interpreted in the past, this must be 'creep' and likely creep to be associated with a movement toward acclimitization. It is noteworthy to have found evidence for it in five hour-experiments. (These are not attempts at farreaching conclusions drawn from only a few hours of data. They are based on hundreds of hours of test over a considerable number of years, with a continued sifting of ideas before their current very tentative gestation.)

In Fig. If are presented the results of graphically differentiating the tank pressure-time decay of ventilating air drawn from a known high pressure tank volume of compressed breathing quality dry air. For each of the four temperature experiments described in Fig. 1a, the data are first presented as the raw rate of change of volume per unit measuring interval. The second curve for each experiment then smooths the derivative data. The approximate range of the highest frequency cycle is about 1300 - 1800 seconds per cycle, centered around 1550 seconds; thus agreeing with the temperature data. Filtering these curves results in the next set which show a cycle time in the 
range 4000 - 5300 seconds per cycle, centered around 4800 seconds. The temperature data were centered around 4200 seconds. A next step of filtering reveals a cycle time range of 9500 - 15,000 seconds per cycle centered around 12,000 seconds. The temperature data were centered around 9500 seconds. The next step of filtering represents the means that fall within the accuracy ranges estimated in (2).

The amplitude range, the ratio of maximum to minimum ventilation rates found during the test period, averaged around 3 to 1 .

Thus in summary, a considerable degree of consistency is shown between the ventilation-metabolism data in (4), from Goodman's ventilation data (8), and now in both ventilation and temperature data.

There is also a second set of experiments that were done and described in (2) and designed to show the high frequency states.

Tke experimental problem that had to be faced was to obtain data that were reasonably certain as far as their means and lower frequencies were concerned (i.e. that would agree with the data thus far presented); that were obtained with a high degree of constancy of input conditions; that could present the dynamics of all the stations (so that any one station could not be regarded as special); that would be taken during and representative of a five-hour test period; and that could be done inexpensively. (We have strong antipathy to doing exploratory research in very elaborate equipment setups). The simplest satisfactory choice that could be visualized was a serial slow scanning from station to station, as in the previous experiments, with detailed bursts of data taken, each in turn, at each station. In any five hour experiment, each station was scanned at the rapid rates at least twice, and the slower rate quite a few times less than at the slow rate alone.

As indicated in (2), the means and lower frequencies found were essentially in agreement for the slower components in the slow and fast scan experiments. Therefore, there is nothing to gain in presenting the same massive dose of data shown in Figs. la-f, Instead, only the high frequency data are shown in Fig. $1 \mathrm{~g}-\mathrm{i}$ for temperature, and Fig. $1 \mathrm{j}$ for ventilation rate.

The only points of interest at present are the approximate grand average temperature cycles found in the 80-190 seconds range, centered around 120 seconds, and a cycle in the 275-550 seconds range, centered around 400 seconds. The approximate grand average ventilation cycle was found in the range 90140 seconds, centered around 115 seconds. It was not possible to infer the longer near 7 minute cycle because the decay segments of data were too broken up. The amplitude magnitude for the high frequency temperature cycles is about $1 / 4$ to $1-1 / 2^{\circ} \mathrm{C}$. double amplitude, and its nominal magnitude of maximum derivative is about $\pm 1{ }^{\circ} \mathrm{C} / 100$ seconds, or $\pm 40^{\circ} \mathrm{C}$./ hour. The approximate mean ratio of maximum to minimum ventilation rate shown in the high frequency cycle: is about 3 to 1 . All of the rest of the data are similar in harmonic content to the slower data. 
Thus, in summary, it has again been demonstrated that there is a rich spectrum of oscillator cycles in ventilation and temperature, and thus in metabolism. This demonstration (particularly in temperature which represents heat flux) contradicts current modelling efforts that have tried to establish ventilation as an autonomous oscillator, or as an autonomous system, independent of the power producing system. The coupling between the two systems must be fully explored, and the major problem at issue is the nature of the physiological elements such as the action of receptors, the signalling products of the engine system, the reality of the muscle engine system, and such questions as whether a hormonal signalling system is involved or not.

To summarize the numbers, the demonstrated cycles above the breathing rate are:

(4)

\begin{tabular}{ccc}
$90-120$ & \multicolumn{2}{c}{ seconds } \\
$400-500$ & $"$ & $(?)$ \\
$1000-1300$ & $"$ & $(?)$ \\
$2000-2500$ & $"$ & \\
$15,000-18,000$ & $"$ &
\end{tabular}

(8)

$\begin{array}{cc}1 & \text { minute } \\ 4 & " 1 \\ 20 & " \\ 40 & " \\ 2-1 / 2 & \text { hours }\end{array}$

\begin{tabular}{rcrc} 
& \multicolumn{3}{c}{ Present } \\
120 & \pm & 40 & seconds \\
400 & \pm & 150 & $" 1$ \\
1500 & \pm & 300 & $"$ \\
4500 & \pm & 1000 & $"$ \\
12,000 & \pm & 3000 & $"$
\end{tabular}

Thus, most reasonably there definitely appears to be 1 - 2 minute cycles; 5 - 10 minute cycles; 1ikely a near 20 minute cycle; a 40 - 60 minute cycle; and a 2-1/2 - 3-1/2 hour cycle. It is proposed that the first is an engine cycle; the second a blood fluid vasomotor cycle, likely for heat exchange regulation; the third gas storage in the tissue $\left(\mathrm{CO}_{2}\right)$; the fourth not known (although it may be interchanged with the third, or stem from related gas storage time delays); and the fifth from heat storage in the body in a temperature regulated system. A hormonal coupling is proposed for the first, a1though evidence has been brought forth that the time domain of instability can be reached in whole or part by gas signals.

The additional one element which has not been explored in the experimental data would involve comparing the mean surface temperature dynamics (obtained by averaging the body stations and taking its derivatives) with the ventilation rate and metabolic rate at the lung entrance. This can provide some information about the lags and unbalance of tissue heat production, oxygen consumption, and the lags to the receptor center or centers (for example, a conventional interpretive assumption may be that there is no storage of $\mathrm{O}_{2}$ in tissue as compared to storage of $\mathrm{CO}_{2}$ in bicarbonate in the blood). However, the task is quite time consuming and must be bypassed at present.

The only simple over-al1 assumption one might note, by intercomparison in Fig. 1, is that there is a vague general slow synchrony between temperature and ventilation (and therefore oxygen consumption at the lung, and likely oxygen consumption in the tissue). However, these remarks are no substitute for much more rigorous analysis. 


\section{Further. Investigations Into Temperature Dynamics}

The thermoregulation program being explored will involve tracing the temperature and heating production in through the system until the entire regulating or control system is uncovered. Having demonstrated first pe-. riodic dynamics in ventilation, metabolism, and temperature, it has become timely to begin to develop evidence for the reality of the muscle engine cycle. The general thesis, discussed in some detail in (2), is that the chemical thermodynamic engine must after all really be a chemical 'heat' engine, and that it would be impossible to have an engine in which the power source was not marked by a higher temperature. ( A similar philosophy would be contained in the idea that searching a field to localize the source of loudest noise represents a localizing of the source mechanism. One must be careful, in all such cases, to be able to demonstrate that the dimunition of energy arises from dissipative mechanisms.) In particular, a more complex thesis was advanced in (2) that the muscle sheath engine likely forces a flux of temperature into the core conductively, but that a more severe drop (i.e almost a step discontinuity) can take place into the outer insulating sheath. (This was shown in (2) Fig. 5, p. 28 )

For a first elementary exploration of these questions (an elementary exploration was chosen to allow some time to build up experimental techniques and measurements), it was proposed to place thermocouples in a muscle sheath, the core, the insulating layer, and on the surface in some test animals. Some work, preliminary to the problem, was done on white mice, guinea pigs, and humans; and on the problem, in mice and guinea pigs.

For example, as a first illustration, consider some skin temperature data taken on mouse, guinea pig, and human. These are shown in Fig. 2. These data represent, as a first illustration, some typical data taken on the test 'animals' (i.e. just as they were taken for Fig. 1 in the previous section) that show some of the local surface dynamics.

What is clearly evident is that a near 1-2 minute cycle is obtained for mouse, guinea pig, and human, with no size scaling law. Here one may note (subject to demonstration on many other types of warm-blooded animals, or perhaps only mammals) that just as the oscillator frequency domain in the nervous system is essentially independent of animal or animal size (at present numbers up to a factor of two or so are to be disregarded), or the heart pump oscillator frequency domain, much more crudely, lies in a particular range, here the metabolic 'engine' cycle frequency is found to be similarly independent of mammal size. It is thus unlikely to attribute this fundamental oscillator cycle to a time delay in the gas absorption ratio (one may note in closer conformity with the facts that breathing frequencies essentially change with animal size to accommodate the gas ventilation rate and that the heart rate changes to accommodate the oxygen need). One may also note that the amplitude excursion, as well as the derivatives are essentially similar.

What had been expected in (4) was that the long thermal time constant (i.e. three hours) for the system was approximately independent of size for the reasons that the body thermal capacitance was lumped (because of the fast 
internal blood distribution system), and the thermal resistance was lumped as a surface resistance (being predominantly an air boundary resistance). If both the metabolism per unit area was nearly the same for large and small animals (as Herrington's data (28) on rat, guinea pig, and human seemed to bear out), and if the resistance law were also proportional to surface area, then the thermal time constant in air would be approximately the same for large and small animals. (It would not be so in well-stirred water in which the volume conductance could finally govern; or it would not necessarily be true in very small animals in which the boundary conductance type changed.) Thus, one has to visualize a possible spectrum of effects in time scale between the breathing cycle and a long, near-three-hour thermal cycle. Here begins the evidence that the time domains are likely to be similar, and arise from isomorphic mechanisms which cause the non-linear cycles, in which some are dependent on size and others not. In these first data, the engine cycle is independent of size. This represents one additional piece of evidence that brings the problem closer to involving a motor unit hormonal interaction.

For a second round of experiments, a probe of temperature with depth was attempted. Practice on a mouse's muscle served as preparation for implantthermocouples into a guinea pig.

The thermocouples used were 0.010 inch wire copper-constantan thermocouples made by Omega Engineering, Inc. While the wire size was somewhat coarser than had originally be planned on, the company makes this thermocouple with just the property that was desired, namely a $1 \mathrm{~mm}$ (i.e. 0.040 inch) spherical end.

The reason for the $1 \mathrm{~mm}$. temperature sampling size is the following: in Newburgh (29), work of Bazett and McGlone is reported (on p. 132), on a depth survey in forearm and thigh of a human through the outer insulating layer (namely in depths up to $24 \mathrm{~mm}$.). It is quite clear that the temperature gradient is quite constant on the scale of the entire sheath. Yet on p. 170 in a much finer survey within the first few $\mathrm{mm}$. and with a resolved sensitivity of $0.1 \mathrm{~mm}$., local variations in temperature are shown that reflect passage past venous and arteriolar elements which act as heat exchangers at the $0.1-0.2 \mathrm{~mm}$. size level. If the gradient from p. 132 is laid on p. 170, one suspects that by averaging over about $1 \mathrm{~mm}$. spheres these fine point variations would be washed out. Further it is desirable to use a field sampling size that will wash out high frequency time variations of temperature. Again, a $1 \mathrm{~mm}$. bulb size with liquid immersion suits detection of 100 seconds cycles without over-emphasizing higher frequency noise. (A $6 \mathrm{~mm}$. bulb commonly requires about a three-minute response time. Since the response time varies as the square of the diameter, a $1 \mathrm{~mm}$. bulb has a likely response time in the few seconds range.)

Three thermocouples were placed into a guinea pig as follows: They were placed approximately on a line in a plan view, each about $1 / 4$ " apart in the posterior right leg in the region of the gracilis posticus, and semitendinosus muscles. (This region was chosen to be a region of large muscle size, with a large core, and free of internal organs.) The deepest thermocouple was placed about $1 / 2^{\prime \prime}$ in from the skin directly anterior to the semitendinosus muscle. 
(The muscles were bisected and the thermocouple slipped under the muscle.) The second was sutured to the plane of bisection of the two muscles at a depth of about 1/4". The third was placed directly on top of the gracilis at a depth of about $2 \mathrm{~mm}$. Intermittently, skin temperature measurements were also made in the same general region. Thus, the three thermocouples represent sources of data, in a crude way, from the core, from the muscle layer, and from the region exterior to the muscle layer.

Fig. 3 shows the response from these three layers. Data were taken on successive days after the operation to assurc some reliability that the data were representative of the animal rather than of the operative upset.

During each experiment the animal was quiescently penned in a large plastic tube and supplied with food and drink, of which he occasionally partook. Data on the entire experimental sequence are not shown, solely for the sake of brevity. Instead, only a number of illustrative examples are depicted. The entire sequence consisted of:

\begin{tabular}{|c|c|}
\hline Date & $\underline{\text { Run }}$ \\
\hline $2-14-64$ & Implantation performed. \\
\hline $2-17-64$ & $\begin{array}{l}3 \text {-hour run on all three stations; serial reading at } \\
20 \text { seconds interval. }\end{array}$ \\
\hline $2-18-64$ & $\begin{array}{l}\text { Same as } 2 / 17 / 64 \text {, but followed by a } 2500 \text { second segment } \\
\text { scanning at } 10 \text { seconds interval. }\end{array}$ \\
\hline $\begin{array}{l}2-19-64 \\
2-21-64\end{array}$ & $\begin{array}{l}\text { 5-hour run, scanning serially at } 20 \text { seconds. } \\
\text { 1-hour run; differential measure between muscle tem- }\end{array}$ \\
\hline $2-22-64$ & $\begin{array}{l}\text { perature and core temperature, } 20 \text { seconds scan. } \\
\text { Three one-half-hour runs; one for skin temperature, } \\
\text { one for muscle-core difference, one for muscle- } \\
\text { outer layer difference; } 20 \text { seconds scan. (The } \\
\text { skin temperature station was slightly dorsal to } \\
\text { the incision.) }\end{array}$ \\
\hline
\end{tabular}

What is clear in these data is that the 1-2 minute cycle continues at al1 stations. (This is in agreement with the experiments performed in (4) in which the harmonic content of a station was found to be the same at all stations, whether skin, under the arm, mouth, urethra, ear, esophagus, or rectum.)

Second, it was found that the muscle sheath produced temperatures higher than the core by about $0.3^{\circ} \mathrm{C}$. Since a direct comparison of the data from each station does not show the results so clearly, the thermocouples were re-connected differentially so that the muscle sheath-core difference was measured directly. This clearly showed a mean $0.3^{\circ} \mathrm{C}$. difference.

Third, with a mildly cooled exterior (i.e. in a situation in which the skin temperature was dropped to about $\left.36^{\circ} \mathrm{C}.\right)$, a difference of about $1^{\circ} \mathrm{C}$. was obtained between the muscle sheath and the layer external to the muscle sheath.

In a rough fashion then, the nominal picture looks like the following: 


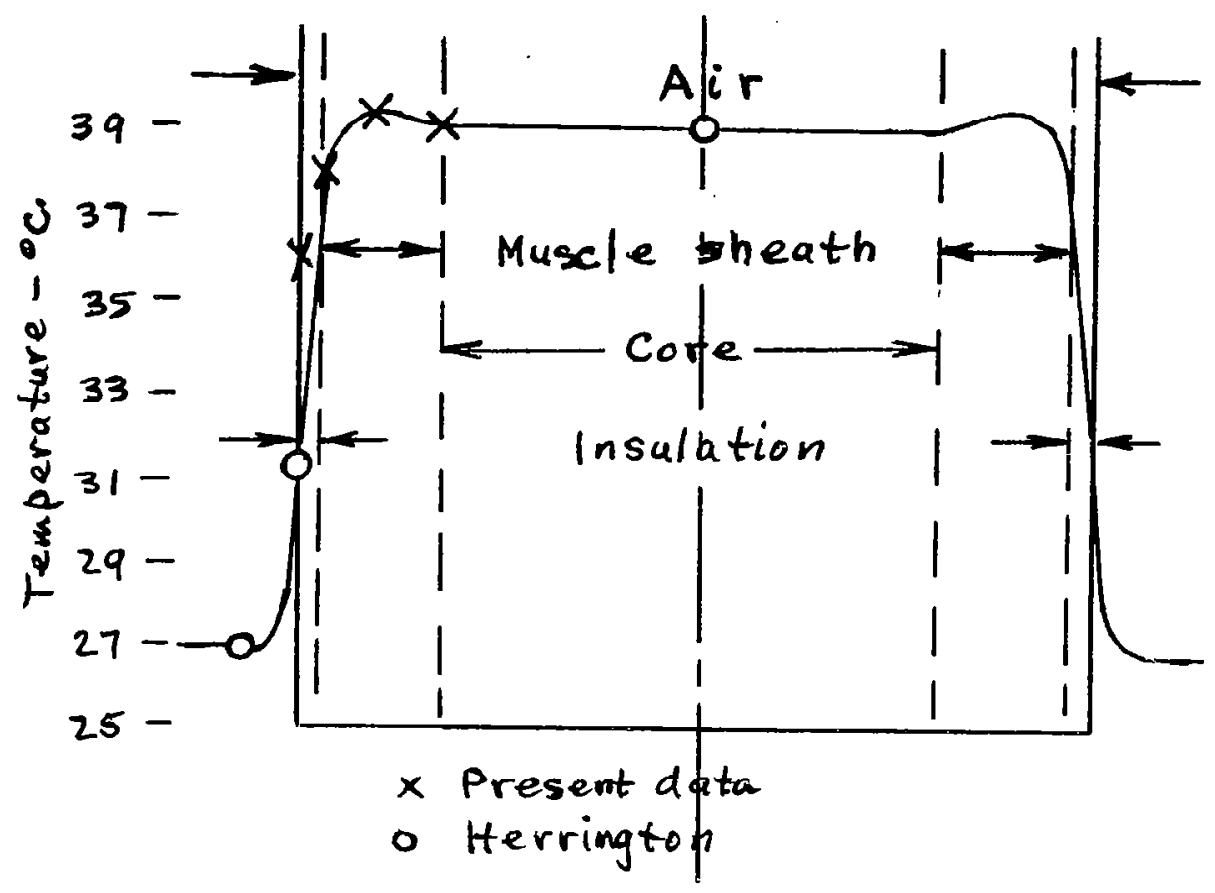

From this picture, a very crude estimate can be made of the power production in the muscle layer. At the inner boundary, a rough estimate of the gradient of about $4^{\circ} \mathrm{C}$. per inch was made, and at the outer boundary of about $30^{\circ} \mathrm{C}$.per inch, for a sum of about $30^{\circ} \mathrm{C}$. per inch. (About $10 \%$ of the flux is inward, which would make sense since the inner core has to supply the heat flux to evaporate water and heat air in the breath).

The thermal conductivity of tissue may be chosen (see (29) p. 89 for example) between the limits of

$$
\mathrm{k}=0.0005 \mathrm{cal} \cdot / \mathrm{sec} \cdot \mathrm{cm} \cdot{ }^{\circ} \mathrm{C} \text {. }
$$

quoted for muscle and fat tissue, and

$$
\mathrm{k}=0.0014 \mathrm{ca} 1 . / \mathrm{sec} \cdot \mathrm{cm} \cdot{ }^{\circ} \mathrm{C} \text {. }
$$

for water, with the former number having been also estimated as suitable for tissue. Thus,

$$
\begin{aligned}
\frac{M}{A} & =0.005 \frac{\mathrm{cal} ;}{\mathrm{cm} \cdot{ }^{2} \mathrm{sec} .} \\
& =200 \frac{\mathrm{k} c \mathrm{cal} .}{\mathrm{m}^{2} \mathrm{~h}_{\mathrm{r}} .}
\end{aligned}
$$

Since the over-all heat prodyction for guinea pigs (see (28) p. 449 for example) is about $40 \mathrm{k} \mathrm{cal} . / \mathrm{m}^{2} . \mathrm{hr}$., the best one can say is that an answer agreeing to within an order of magnitude has been found. No claim is made that the experimental 'estimate' is accurate or inaccurate. If 
inaccurate, then a much more detailed internal survey is needed to establish quantitative reliability. The present 'experimental' result are only regarded as being a semi-quantitative demonstration that heat power is likely produced in the muscles to heat the body.

Once again the 1-2 minute cycle makes itself evident, and once more it is even less convincing that the cycle originates from gas equilibrium lags. The signals must result from the by-products of oxidation. Whether they arise from one of the intermediate steps in the long chain of biochemical oxidation, or by a trigger - such as a hormone - produced at some step in the chain is not clear.

The availability of temperature data from a number of stations and animals permits demonstrating a little more evidence on the cyclic responses of the system. Thus, the following experimental log illustrates a variety of temperature measurement experiments in which are noted some of various cycles that have been and can be pencil-filtered out of the data.

\section{Some human data}

Two tests to see effect of neighboring muscle masses

1. Jan. 21 - Exp. 5 - quiescent subject SC, normally dressed - left armpit temperature (arm closely folded) - test period $4000 \mathrm{sec}$ - $15 \mathrm{mg}$. isuprel given at $2100 \mathrm{sec}$. (subject normally uses drug for asthma).

Mean temperature $35.7^{\circ} \mathrm{C}$. Weak waves found; double amplitude $\sim 0.15^{\circ} \mathrm{C}$. Cyclic periods: $\sim 100 \mathrm{sec}$; $0-2000 \mathrm{sec} ., 180 \mathrm{sec}$; $2000-4000 \mathrm{sec}$, $280 \mathrm{sec}$.; weak $2600 \mathrm{sec}$.

2. Jan. 22 - Exp. 6 - quiescent subject SC, normally dressed - between buttock cheeks, 1 inch in fold - test period $4000 \mathrm{sec}$ - $15 \mathrm{mg}$. isuprel given at $1800 \mathrm{sec}$.

Mean temperature $35.7^{\circ} \mathrm{C}$; double amplitude waves $\sim 0.3^{\circ} \mathrm{C}$. Cyclic periods: $\sim 115 \mathrm{sec}$; $0-2000 \mathrm{sec}$. weak $480 \mathrm{sec}$.; 2000-4000 sec., weak $690 \mathrm{sec}$.

3. Jan. 22 - Exp. 7 - quiescent subject SC, normally dressed - left index finger - test period $4000 \mathrm{sec}$ - $15 \mathrm{mg}$. isuprel given at $2100 \mathrm{sec}$.

Room tempe rature $24^{\circ} \mathrm{C}$.; Mean test temperature $35.2^{\circ} \mathrm{C}$. Cyclic periods and double amplitudes: $85 \mathrm{sec} 0.2^{\circ} \mathrm{C}$. amplitude; 340 sec., $1^{\circ} \mathrm{C}$. amplitude.

(SUMMARY: 1-1/2 - 2 minute period; 4-8 minute periods are evident) 
4. Jan. 27 - Exp. 8 - quiescent guinea pig - left hind quarter (shaved) test period $2500 \mathrm{sec}$.

Mean temperature $35^{\circ} \mathrm{C}$.

Cyclic periods: $70 \mathrm{sec} ., \sim 0.25^{\circ} \mathrm{C}$. amplitude; $250 \mathrm{sec}$.

5. Jan. 31 - Exp.11 - quiescent guinea pig - thigh (shaved) - test period $12,000 \mathrm{sec}$.

Range $31-35^{\circ} \mathrm{C} . ;$ Mean $33.0^{\circ} \mathrm{C}$.

Cyclic periods: $77 \mathrm{sec}, \sim 0.25^{\circ} \mathrm{C}$. amplitude; $200 \mathrm{sec}, 0.5^{\circ} \mathrm{C}$. amplitude; $570 \mathrm{sec}, 1^{\circ} \mathrm{C}$. amplitude; $1900 \mathrm{sec}$, $0.7^{\circ} \mathrm{C}$. amplitude; $20,000 \mathrm{sec}, 3.5^{\circ} \mathrm{C}$. amplitude.

6. Feb. 3 - Exp.12 - quiescent guinea pig - thigh (shaved) - test period $10,000 \mathrm{sec}$.

Range $32-35^{\circ} \mathrm{C} . ;$ Mean $34^{\circ} \mathrm{C}$.

Cyclic periods: $83 \mathrm{sec}, \sim 0.25^{\circ} \mathrm{C}$. amplitude; $235 \mathrm{sec}, \sim 0.5^{\circ} \mathrm{C}$. amplitude; $620 \mathrm{sec}, \sim 0.3^{\circ} \mathrm{C}$. amplitude; $1400 \mathrm{sec}, \quad 0.5^{\circ} \mathrm{C}$. amplitude; $2500 \mathrm{sec} ., \sim 0.3^{\circ} \mathrm{C}$. amplitude; 10,000-20,000 sec., $2^{\circ} \mathrm{C}$. amplitude.

(SUMMARY: 1-1-1/2 minute period: 4-10 minute periods (possibly two periods); 20-40 minute period (possibly two periods); 3-5 hour period)

\section{Some white mouse data}

7. Feb. 6 - Exp.15 - quiescent white mouse - right hind quarter (shaved) test period $10,000 \mathrm{sec}$.

Range $33-36^{\circ} \mathrm{C}$; Mean $35^{\circ} \mathrm{C}$.

Cyclic periods: $67 \mathrm{sec}, \sim 0.2^{\circ} \mathrm{C} . ; 480 \mathrm{sec}, \sim 0.25^{\circ} \mathrm{C} . ; 2700 \mathrm{sec}$, $0.3^{\circ} \mathrm{C}$.

8. Feb. 7 - Exp.16 - quiescent white mouse - right hind quarter (shaved) test period $12,000 \mathrm{sec}$.

Range $31.5-36^{\circ} \mathrm{C}$; Mean $\sim 34^{\circ} \mathrm{C}$.

Cyclic periods: $70 \mathrm{sec} \sim 0.2^{\circ} \mathrm{C} . ; 320 \mathrm{sec}, \sim 0.25^{\circ} \mathrm{C} . ; 1700 \mathrm{sec}$, $\sim 0.5^{\circ} \mathrm{C} . ; \sim 10,000 \mathrm{sec} ., \sim 2^{\circ} \mathrm{C}$.

(SUMMARY: 1 minute period; 5-10 minute period; 30-50 minute period;

3 hour period) 
Brief comments concerning the cardiovascular section of (2) have been received from Fry who states that "I am not as convinced as you that the evidence for unexplained large damping is so clear-cut. For example, if you will permit a linear analysis of the pressure data along the aorta, the moduli of pressure harmonics will be found generally to increase with distance"......"at least for those that are in the accurate frequency range of the manometer system. Taylor has recently described a tapered transmission line model to explain this." However, there still remains a serious problem of being certain of the data. For example, data such as Patel, Mallos, and Fry (30) showing an increasing pressure along the aortic arch, before the pressure finally decreases is not acceptable to a11.

Dr. Olmsted made the following comments on the Wetterer data presented in (2) p. 61; outside of a little added sharpness required at the tip of the flow curve; a disregard of the wiggles shown after the reverse flow spike in the flow curve as being artifacts; a small step in the middle of the rising portion of the pressure wave; more than one small oscillation at the pressure notch (typically an under-over, or an under-over-under high frequency damped wave of small amplitude); and a somewhat less steep descent from the notch that cuts under the indicated curve, the presentation was on the whole quite accurate. None of these detailed changes would make any noticeable difference in the figure's analysis. Thus, the argument as far as it went in (2) remains unchanged. (Some examples of current records of Dr. Olmsted on intact unanesthetized trained dogs in a quiescent state from the ascending aorta - mean section velocity, pressure, and integrated flow - are illustrated in Fig. 4.) However, the argument must bccome more detailed with regard to the wave downstream of the ascending aorta as one gets further out in the arterial system.

Generally, what is expected is that the pressure wave gradually approa= ches looking more like a damped oscillation with two full lobes superimposed on a triangular wave; that, whereas at the aorta, the pulsatile amplitude is about $40 \mathrm{~mm} . \mathrm{Hg}$, it is likely cut in half at the point at which the internal diameters of vessels are of the order of 0.025 inch; that the mean pressure, however, has not particularly changed; that the wave at these conduit sizes is free of detail except for the 'fundamental' damped oscillation; that it isn't until one gets well down to arteriole size that the pressure begins to change rapidly with length, in that both the pulsatile pressure and the mean pressure begin to drop; that at the drop from a level of about $100 \mathrm{~mm}$ of Hg., roughly half takes place and is partly in the control range of the arterioles, and the other half takes place in the fixed capillary bed; but that the controlled pressure drop level in the arterioles take place more slowly than a few seconds, so that their action cannot be invoked for pressure control during the individual heartbeat cycles.

What do these factors mean physically? First, the fact that the mean pressure does not change much would seem to imply that the system acts very much as if it were a closed system (i.e. as if the mean flow does not have 
a great deal of influence on the pressure drop). The fact that the mean pressure has not particularly dropped at a level at which the tube size is quite sma11, once again implies that the arterial system is not noticeably resistive - the resistance being downstream - although it is actively inertial and capacitative. More specifically, there is no causal fact yet found that suggests that the arteriole - capillary system is anything but fixed (not necessarily linear) resistive, with a little capacitance thrown in. Aptly framing the problem status, as of 1956, and requiring only mild correction, 1s the following from (31).

"Little is known concerning the pulsatile movement of the blood along the aorta, chiefly because of inadequacies and difficulties in applying existing pulsatile flowmeters. Most of our knowledge has been deduced from pressure pulses recorded at various points along the aorta or by use of differential pressure flowmeters."

"No investigator has compared aortic flow pulses with the simultaneous differential pressures existing across the segment of vessel under study; yet, the close relationship between flow and differential pressure has long been recognized. The pressure transmission studies made by Hamilton and Dow demonstrated a system of standing pressure waves in the aorta which they predicted would act on the flowing stream to produce alternate acceleration and retardation. Their concept established the aorta physically as an elastic and relatively frictionless tube. Frank recognized this in his theoretical treatment of the stream of velocity in vessels and stated in his paper 'the pressure difference, which represents the moving force, can, in a frictionless current, only be balanced by forces of inertia.' The exact phase relationship of differential pressure to acceleration in the large arteries was, however, apparently not clear to him."

"This communication will present what we believe to be the true blood flow curves of the aorta and establish their validity by their relationship to the differential pressures existing along that structure."

In correction of this paragraph, the essence of the matter is not that the aorta is frictionless - any tube can be as largely restrictive as one wishes, yet will show no pressure drop at no flow - but that the pressure transmission is not the resultant of a large mean flow being transmitted in a restrictive Iine. As Frank recognized, it acts like a "windkessel", an elastic storage, with a terminal resistance, but because the aortic transmission line has negligible resistance, it is an inertial reaction that must take up the pressure transmission forces. However, Frank also well understood that the combination of inertial reaction and elastic storage create a propagation velocity, and thus the likelihood of resonance. The basic problem of concern is the question of whether this resonant wave is damped by comprehensible mechanisms. Thus, the path chosen in this report will be to seek a more detailed view and review and add more commentary to what has been developed in (2).

The theory that a pulse of flow causes a pulse of pressure because of the elasticity of the arterial tube system and that a pressure wave runs down the 
elastic tube was founded by the Webers, Moens, Korteweg, and others, and improved by Frank. The fact that this pulse wave would give rise to reflected waves and thus, likely, to standing waves was due first to Frank (see (32), or Frank's papers in 1905 and 1930). A thorough investigation of the main standing wave was done by Hamilton and Dow (33) to whom American knowledge of the standing waves is commonly attributed. It appears clear in the work of Frank, Hamilton and Dow, or, more recently, to Alexander (34), Wetterer (35) and Peterson (36), that very commonly the problem of the wave effects has been regarded as a comparison of signal at the upstream end (say in the ascending branch) of the aorta with signal as far down as could conveniently be reached in, say, the femoral artery. As far as the pressure data at these stations are concerned, the fidelity, from Frank's time as compared to the present, was quite reasonable, in that there is not a large amount of improvement in detail in chosing the work of careful investigations past 1930. The flow data, on the other hand (as (31) implies) is more suspect until Kolin's electromagnetic flowmeter.

The question in proposing to view the problem of damping was to find the simplest way to bring the issue into focus. Physically, one is forced to think of a simple modelling, as proposed in (2) but not carried out, in the form of lumped networks. As a matter of fact, it was in preparation for laying a foundation for a future lumped network attack that the previous history was summarized in the papers here referenced and reviewed. It may be that Karreman (37) may have done a considerable amount of the task.

What are the characteristics that have to be accounted for?

1. Although one may assume that the heart injects in a pulse of flow, and that the valves shut off well, there is a pulse of backflow shown at a measuring station in the ascending aorta. This likely requires a portion of axial extensibility of the aorta, relative to a fixed station, as wel $l$ as circumferential extensibility.

2. The generated pressure pulse has a variety of high frequency details at the notch to be accounted for. This requires both high frequency response elements and high frequency damping.

3. The pressure pulse downstream at the femoral artery is quite a bit freer from detail. It shows a lag, the high initial pulse, an apparent wave train and some nominal attenuation of the train.

4. The flow pulse at the femoral artery is approximately a damped oscillation around a mean value. (Clearly seen in the excellent work of Wetterer (35) ).

5. Since there is a phase reversal between the phase of pressure oscillations near the heart and in the femoral artery, there is likely to be a node inbetween "situated in the thoracic or upper abdominal aorta" (according to Wetterer). Thus, according to the ideas of Frank, the effective length of the aorta is half a wave length of the main standing wave. 
The simplest model for such a system, as Karreman (37) undoubtedly visualized, requires two capacitances. To help follow the analysis, a simpler one capacitance model may be first visualized.

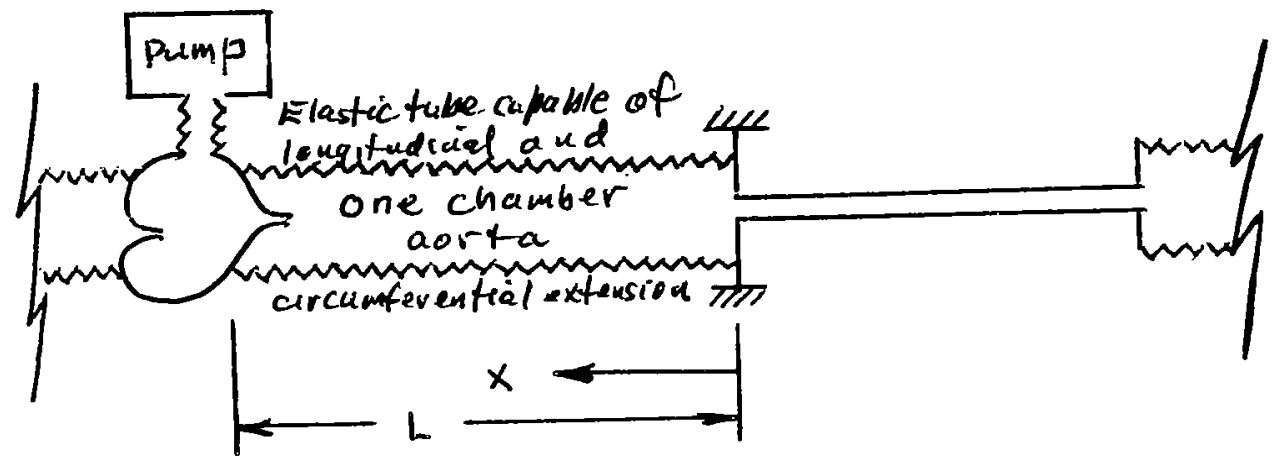

Likely representative of good flow data in the ascending aorta (in addition to Fig. 4) are the following sources (on the dog): Wetterer-1954 (35); Spencer-1958 (38); Spencer-1962 (40); Greenfield et a1 - 1962 (41); and Patel - 1963 (42).

What is first needed is that a monotonic injection of a pulse of volume should produce a negatively overshooting damped oscillatory pulse flow as the valve cuts off. (That the flow cuts off sharply from the valve is borne out by study of the divergence of left ventricular pressure and and aortic arch pressure as can be noted in Spencer (40). The negative flow pulse exists when the ventricular pressure is substantially lower than aortic.) Since the valve is cut off and then the flow oscillation takes place, with a relatively high frequency judged to be about $16 \mathrm{cps}$ (from (38) and (40)) in the dog, and with a damping ratio lying in the range 0.2-0.5 critical damping (judged from (35) and (38). The value is a crude estimate, only because the data are limited, not because the estimate is crude.)

Such characteristics are to be expected from a mass-spring combination. Because of the high frequency, and the essential capacitative nature of the aortic storage, the first lobular part of the aorta might act as a lumped capacitance for this signal. The first problem is to estimate this response. A reasonable guess is that the first lump might be the segment in the ascending aorta up to its bend, i.e. up to a point at which a spring-like reaction might be expected.

For plausibility of this model, it is desirable first to view the flow pulse relation down the system. For example integrating a flow curve in the femoral artery that Wetterer presents (35), just as was done in (2) p. 41, indicates an approximately linear climb in volume with a gentle sinusoidal oscillation (with an amplitude of perhaps $6-10 \%$ of the total volume climb per beat) around the linear increase. Thus, one can estimate how much of the flow pulse has reached the femoral artery at the time the valve closes. In 
particular, at about the time the valve closes, the flow pulse has transmitted about half of its ultimate volume per beat. Under these circumstances, as a first approximation, the flow pulse has charged the line fairly completely. Thus, the backflow must be a volume surge from some portion that has been accelerated, and now a spring return is causing the high frequency oscillation after the valve closes.

The governing compliance should essentially be the entire equivalent lumped compliance in which a change of $30 \mathrm{~mm} . \mathrm{Hg}$. causes a volume change of $8 \mathrm{cc}$. (from Wetterer's data (35)). This value, taken on a $15 \mathrm{~kg}$. dog, compares well with the arterial distensibility that Wetterer shows (Fig.8, (35)), which he obtained from a slow added pump action

$$
\frac{8 \mathrm{cc} .}{30 \mathrm{~mm} \cdot \mathrm{Hg} \cdot}=\frac{8 \mathrm{cc} \cdot}{30 \mathrm{~mm} \cdot} \quad \frac{760 \mathrm{~mm} \cdot}{1.013 \times 10^{6}} \frac{\text { dynes }}{\mathrm{cm}^{2}}=0.20 \times 10^{-3} \frac{\mathrm{cm}^{3}}{\frac{\text { dynes }}{\mathrm{cm}^{2}}}
$$

Fig. 8 (35) shows $0.2 \times 10^{-3}$ "cgs" units

(tig. 8 in (35) really proposes that much of the dynamic events in the hydrodynamics of the cardiac cycle should be scaled by animal weight. For example, the figure includes a datum on the human.)

It is clear that the effect of pumping is to accelerate some fluid, some aortic mass, and some attached tissue to which the aorta is tethered, and that there are spring rates associated with these displacing parts. The aorta approximately pressurizes as a whole (the pressure also in the femoral artery has peaked by the time the valve closes), but it is not clear what the volume elastic deformation modes are (and there could be many). By physical intuition, one might assume that a bending mode, in which the ascending aorta tends to straighten out (i.e. a Bourdon tube bulging function) by virtue of bulging, is the high frequency pulse forming mode. Time does not permit a fuli discussion of Bourdon tube theory. (Illustratively, see Jennings "Theories on Bourdon Tubes" (43)). However, a quick crude estimate can be made as follows:

$$
\mathrm{T}=\mathrm{I} \frac{\mathrm{d}^{2} \boldsymbol{\theta}}{\mathrm{dt}^{2}}
$$

expresses the dynamic bending equation of motion.

$\mathrm{T}=$ torque required to unbend the aortic arch

$\theta=$ angle of unbend of the arch

$I=$ mass moment of inertia of the arch as a tube of fluid and wall

$$
T=E I^{\prime} \frac{\Delta R}{R^{2}}
$$

expresses the restoring 'spring' torque required to deform the arch. 
$\mathrm{E}=$ elastic modulus of the tube wall

$I^{\prime}=$ area product of inertia of the arch

$\Delta R=$ change in arch radius produced by torque

$\mathrm{R}$ = radius of arch

$$
\frac{\Delta R}{R}=-\frac{\Delta \theta}{\theta}
$$

$\mathbf{\Delta} \boldsymbol{\theta}=$ angular deformation of arch

$\boldsymbol{Q}=$ angle of $\operatorname{arch}\left(\mathrm{e} \cdot \mathrm{g} \cdot 180^{\circ}\right)$

The inertial moment of inertia and the area moment of inertia are not equal, one being proportional to the mass, the other the area. As an approximation, assuming an elementary result that would be true for rectangular cross-sections

if

$$
\begin{aligned}
& I=\Sigma m r^{2} \\
& I=\Sigma A r^{2}
\end{aligned}
$$

then

$$
\frac{\mathrm{I}}{\mathrm{T}^{i}}=\frac{\rho(\mathrm{AD})}{\mathrm{A}}=\rho \mathrm{D}
$$

$A=$ cross-sectional area

$\mathrm{D}=$ diameter

$\rho=$ density

Thus,

or,

$$
\begin{aligned}
\frac{d^{2} \theta}{d t^{2}} & =-\left(\frac{2 E}{\rho D^{2} \theta}\right) \Delta \theta \\
\omega_{0} & =\sqrt{\frac{2 E}{\rho D^{2} \theta}}
\end{aligned}
$$

$\omega_{0}=$ natural frequency of this harmonic oscillator

The elastic modulus $\mathrm{E}$ may be estimated as follows: In McDonald (44), p. 170, results of Bergel give an elastic modulus of about 6 atm. $=90$ psi. In (2) the modulus-thickness product was estimated to be about $8 \mathrm{psi} \mathrm{cm}$. from the data of Wetterer on about a $15 \mathrm{~kg}$. dog. McDonald (44) p. 161 estimated the wall thickness to diameter ratio to be about 0.08 . In Patel et al (42), the approximate diameter of the aorta in dogs is shown to be about $3 \mathrm{~cm}$. The wall thickness is thus estimated to be about $1 / 4 \mathrm{~cm}$. The elastic modulus is thus about $30 \mathrm{psi}$. At present, as an approximation only, a mean value of about 50 psi can be adopted. Thus,

$$
\begin{aligned}
& E=50 \mathrm{psi}=3.3 \times 10^{6} \text { dynes } / \mathrm{cm}^{2} \\
& P=1 \mathrm{gr} . / \mathrm{cm}^{2} \text { (water-like density) } \\
& D=3 \mathrm{~cm} . \\
& \theta=\pi \text { radians (a half circular arch assumed) }
\end{aligned}
$$




$$
\begin{aligned}
& \omega_{0}=480 \text { per sec。 } \\
& f_{0}=80 \text { cps }
\end{aligned}
$$

$f_{0}$ - natural frequency

This frequency is too high.

Alternately, one may consider the total elongation mode of the system in which its circumferential extension is accompanied by a longitudinal extension. However, this case needs some discussion.

What would not be so immediately obvious is that the lowest frequency response of the system may very well be due to its wall compliance. Contributing to the isolation of this factor is the fact that the walls tend to be longitudinally tethered. Thus, the lowest frequency may be the MoensKorteweg resonant frequency. In (2) p.55, a crude estimate of the MoensKorteweg resonance was about $4 \mathrm{cps}$. Spencer et al in (38) estimated the resonance to fall in the 3-6 cps range, with a mean of 4.5 cps. Alexander (34) shows frequencies in the range 4-6 cps, etc. Thus, for practically al1 purposes, the circumferential compliance, the fluid inertia, and the finite nearly terminated length of the large aortic tube creates the fundamental 'organ pipe' resonance of the arterial system.

Ordinarily, in elastic systems, the longitudinal and circumferential stresses and strains are simply related by a second elastic parameter, such as Poisson's ratio (or any second modulus). Usually, the range is so moderate and limited for Poisson's ratio that generally a length mode (say in bending, or elongation) might be expected to be the low frcquency modc. In the arterial system, it is common to assign the system a tissue value for Poisson's ratio of $1 / 2$ (42). These numbers, $E=50$ psi, $\boldsymbol{\nu}=1 / 2$, thickness $\sim 1 / 4 \mathrm{~cm}$., length $\sim 40 \mathrm{~cm}$. would be suited to a very similar rubber tube, which has therefore been commonly used as a model.

However, the tube is tethered. While there is considerable discussion about the longitudinal elongation, the most unequivocal results appear to be in a relatively recent paper (30), although there is considerable discussion in McDonald (44). Patel et al (30) and (42) results seem to indicate the following. A pressure pulse in the aorta causes an in-phase increase of aortic diameter at any point in the aorta and an elongation of aortic portions of length in phase with the pressure in the early part (the abdominal part) of the aorta. (There are elastic deformations that take place and are relatcd to the crude slower breathing frequency, involving the entire deformation of the thoracic and abdominal regions, but these are not of concern to the dynamics within the individual heat beat cycle.)

These results would seem to indicate that the circumferential deformation and longitudinal deformation tend to beconsiderably decoupled. Specifically, if the Poisson's ratio of the tube tissue is nearly $1 / 2$, i.e. it behaves nearly like an incompressible fluid rather than a solid, then the following deformations may be estimated for a cylinder capped at both ends subject to a uniform internal pressure. (See Prescott (45),p.331.) 


$$
\begin{aligned}
\alpha & =\operatorname{radial} \text { strain } \\
& =\frac{p}{E\left(b^{2}-a^{2}\right)}\left[a^{2}(1-2 \nu)-\frac{a^{2} b^{2}}{r^{2}}(1+\nu)\right] \\
\beta & =\text { circumferential strain } \\
& \left.=\frac{p}{E\left(b^{2}-a^{2}\right)}\left[a^{2}(1-2 \nu)+\frac{a^{2} b^{2}}{r^{2}}+\nu\right)\right] \\
\gamma & =\text { longitudinal strain } \\
& =\frac{p}{\text { E }\left(b^{2}-a^{2}\right)} \\
b & =\text { outer radius } \\
a & =\text { inner radius } \\
r & =\text { radial variable } \\
P & =\text { pressure } \\
S & =\text { wall thickness }
\end{aligned}
$$$$
\mathrm{a}=\text { inner radius }
$$$$
\mathbf{p}=\text { pressure }
$$

For Poisson's ratio near $1 / 2$ and thin wall tubing

$$
\begin{aligned}
& \alpha=-\frac{3}{8} \frac{p}{E} \frac{D}{S} \\
& \beta=\frac{3}{8} \frac{P}{E} \frac{D}{S} \\
& \gamma=(1-2 \nu) \quad \frac{P D}{4 E S} \approx 0 \frac{P D}{4 E S}
\end{aligned}
$$

One would first note that the longitudinal strain is expected to be very small compared to the circumferential strain. That this is in fact true is borne out in (30)。

Thus, the following thin wall result may be assumed for the aorta. The circumferential stress and strain, which essentially governs the frequency of the Moens-Korteweg wave that is propagated down the arterial system, is given nearly by

$$
\begin{aligned}
& \sigma=\frac{3}{8} \quad \frac{D}{S} P \\
& \epsilon=\frac{3}{8} \quad \frac{D}{S} \quad \frac{P}{E}
\end{aligned}
$$

The longitudinal stress and strain, more particularly longitudinal stretching and straightening forces and deformations, are a much more indeterminable complex. In the first place, the strains are near zero because of 
the value of Poisson's ratio. This is further more nearly true because of tissue tethering. In the third place, there are deformations in the system, such as buckling forces, and bowing in the heart wal1, that represent further interactions with the longitudinal stress system. A source of these complexities is discussed in (30), (44), and (46).

The net effect appears to be that longitudinal 'strains' appear to be much smaller than the circumferential strains. Thus, the relationship

$$
\epsilon=\frac{(1-2 \nu)}{4} \frac{P D}{4 E S}
$$

would appear to be meaningless. One could use only experimental data to estimate an effective Poisson's ratio. With the Patel et al value (30) of a length change in the thoracic aorta about one-sixth as much as the change in diameter for deformation with pressure, the longitudinal frequency would appear to be 2-3 times the circumferentially derived frequency. Van Citters (46) gives a theoretical estimate of 7 times, and an 'experimental' estimate of 5. Thus, frequencies in the range 2-6 times the fundamental are conceivablc, or 8-24 cps. This appcars to center validly around the $16 \mathrm{cps}$ estimated at the incisura notch.

Thus, judging the case from Patel et al (30), the situation is viewed as follows. By the time the flow impulse has been passed into the aorta, it has developed the pressure pulse by the R-C system characteristics, and has substantially charged up the pressure in the system, at least down to the femoral artery. However, as the valve slams shut, there is a longitudinal column oscillation, most likely from a diaphragm-like deformation of the valves in the heart wall (i.e. within the first ring in the aorta) at a higher frequency. The column of fluid likely acts quite as a lumped element (as if it involved only some indeterminate length that perhaps descended on $1 \mathrm{y}$ as far as the animal's diaphragm).

This explanation thus attempts a mechanism for the flow and pressure of the incisura by relating it to indeterminately tethered longitudinal motions and to indeterminate but considerable damping associated with this relatively high frequency ( $16 \mathrm{cps}$ ) oscillation. Thus, one can proceed in a simple model by assuming that the input flow is not just a forward volume pulse, but is the experimental value, we 11 demonstrated by Wetterer's data or Olmsted's data, etc. of a pulse and an oscillation at the notch. It is then possible to re-examine the structure of the response shown in (2), the windkessel response shown on $\mathrm{p} .41$, for it is quite clear that the pressure pulse at the aorta entrance does not follow the volume pulse perfectly, particularly down through the pressure notch. (This is not done at the present because sufficient precise data are not at hand at the time of writing to warrant the study.) While not proven with any precision, the eye can judge that the main item missing is likely to be a single high frequency oscillation around the volume output to form the pressure output. It is this high frequency damped longitudinal elastic element at the inlet which may nearly account for the input transformation. 
The modelling may be continued by considering the propagation of this now slightly decoupled pressure and flow wave at the inlet downstream into the system. As Patel et al show in (30) and (42), it is about $40 \mathrm{~cm}$. to the end of the aortic tree in an average dog (i.e. the end of the thoracic and abdominal aortas); and as Remington and Wood indicate (47), it is also about $40 \mathrm{~cm}$. to the femoral artery at the groin in man. In both dog and man, according to these references, there is not much difference in the pressure pulse in that $40 \mathrm{~cm}$. aortic tree. (One argument encountered after preparing (2) was that the end signals in the arterial system are appreciably different from that of the entrance. Yet, referring to these reference did not resolve the argument. Now the reconciliation of the difference begins to become clear. In the aortic tree in the thoracic and abdominal aortas, as shown in Fig. 5 in (42), there is little difference in pulse shape. The changes appear to lie in the arterial branches after they leave the tree.) The pressure wave appears to be an approximate saw-tooth wave, consisting of a steep input ramp and a less steep droop, with a high frequency damped oscillation around the notch. Further down the tree the notch is fairly well damped out. There appears to be a delay of perhaps 0.05 - 0.10 second from the entrance to the aorta to its more terminal end. With a Moens-Korteweg propagation velocity of about $500 \mathrm{~cm} . / \mathrm{sec}$. and a $40 \mathrm{~cm}$. distance, a time delay of about 0.08 second is to be expected. This agrees with the experimental data. Thus, one might expect that the pulse of flow, transformed by the windkesse1 into a pulse of pressure, is propagated down to the end of the line.

The element that must be noted is that the initial flow pulse persists for about 0.15 seconds, which is sufficient for a substantial build up in pressure in the aortic tree (i.e. a pressure build up for about 0.05 second with a time delay of about 0.08 second.) Thus, one might expect, at most, soft remnant $4 \mathrm{cps}$ oscillations superimposed. Since the flow pulse represents a fundamental of the order of $6-8 \mathrm{cps}$, there is 1ittle resonance possible from the fundamental during the open valve phase. There may be a little longitudinal high frequency pulsation of about $16 \mathrm{cps}$ around this flow pulse phase, and in fact some of the more precise pressure data may show the likelihood of steps of this sort of frequency and notches in the steep input front and in details in the notch. That this high frequency is dampened out by a variety of possible mechanisms is likely. Thus, strong resonances are not expected in the short flow pulse phase in the aorta tree.

When the valve closes, the high frequency longitudinal oscillation makes its appearance at the upstream end of the aorta as a damped notch in pressure and overshoot in flow. This high frequency pulse is essentially dampened in the aortic tree.

As an approximation, what is seen in the arterial system is what is depicted by Patel et al (30) as the source characteristics along the aortic tree in their Fig. 9. This figure is reproduced as part of Fig. 5 in this report, which indicates the nature of the source characteristics along the aortic tree. (Data are also used from such sources as (48-54)). The characteristic found is basically a near triangular pressure wave with a time delay and a dampened notch as one gets further down the tree. This can be best seen by comparing original data with the same data smeared over and filtered free of the notch and dicrotic waves. 
If now such source characteristics areapplied to a large number of branches (typically Fig. 5 in Ref. (42) can be used as a somewhat precise picture of the source geometry), such as twenty-nine branches shown in (42), then one can begin to grasp the system characteristics. These branches, of which the femoral artery is illustrative and for which Wetterer indicated a mean efflux flow of about $1.4 \mathrm{cc} / \mathrm{sec}$. as compared to a total cardiac output of about $35 \mathrm{cc} . / \mathrm{sec}$, may be viewed as a general image of about 30 outputs, each generally similar to the femoral artery without straining at reality.

The flow characteristic, as corrected by 01msted, is a highly dampened negative pulse of flow for an instant, with essentially zero flow after the first negative pulse. Thus, the flow reservoir for al1 of these branches past valve closing is the elastic storage in the aorta, i.e. what has been stored in the aorta reservoir at valve closing.

Now the problem that has been posed in (2) begins to have an answer. There is no essential damping in the aortic system (at the primary heart beat frequency, and the $4 \mathrm{cps}$ Moens-Korteweg aorta fundamental. Howcver, the 16 cps dichrotic notch is fairly damped by longitudinal dissipative forces)。 The damping likely resides in the many arterial branches.

The most elementary way to see this is by means of transmission Iine theory, as was invoked in (2). The hydrodynamic transmission line characteristic is determined by a parameter (Womersley's $\alpha$, Iberall's $z, \alpha^{2}=$

$z$ ) $z$ such that if $z$ is greater than 100 , the line is underdamped and almost perfectly resonant (the $Q$ is quite high), whereas if $z$ is less than 1 , the line is overdamped (essentially resistive-capacitative). If $z$ is intermediate, say such as $z=6(\alpha=2.5)$, then the damping would be similar to a lumped system with a damping ratio of about 0.5 (Up to the first resonance, there is little difference in the appearance of the characteristics of a lumped and distributed line.) McDonald (44) has tabulated some values of $z$. For dogs these are

\begin{tabular}{lrrr} 
& \multicolumn{1}{c}{$\alpha$} & \multicolumn{2}{c}{} \\
\cline { 2 - 3 } & $8-11$ & $65-120$ \\
Aorta root & $1.6-3$ & $2-$ & 9 \\
Saphenoul artery & $<1$ & $<1$
\end{tabular}

An approximate summary is that the aorta is undamped, as suspected in (2), and that the arteries indicate various degrees of damping, likely mostly overdamped, except in the cases of larger arteries for the segment up to a first branching. by

As far as the propagation velocity is concerned, it is given approximately

$$
c_{0}=\sqrt{\frac{E S}{\rho D}}
$$




$$
\begin{aligned}
\mathrm{C}_{\mathrm{O}} & =\text { velocity (Moens-Korteweg) } \\
\mathrm{E} & =\text { elastic modulus of wall material } \\
\rho & =\text { fluid density } \\
\mathrm{S} / \mathrm{D} & =\text { wall thickness to diameter ratio }
\end{aligned}
$$

As McDonald indicates (44, p. 161) the thickness to diameter ratio remains fairly constant in the aorta and in arteries, possibly down to but not including arteriole size. Thus, the propagation velocity remains fairly constant. However, the question is what will be the predominant frequency in such arteries?

It is clear that what is happening basically is that the pressure in the aorta decays as it feeds the many arteries (after the valve closes). Each of the arterial systems likely has a higher natural frequency than the aorta (as organ pipes, whether damped or not, they are shorter than the aorta's $40 \mathrm{~cm}$.). However, this aortic supply source stirs up a transient dynamic response in both pressure and flow in the arteries. For example, in the femoral artery, which is most commonly cited, the pressure and flow show a dampened character. The predominant frequency is at or near the Moens-Korteweg frequency and is generally cited as evidence of a resonant standing wave. The pressure peak is higher than in the aorta. The flow oscillation is similar to the pressure oscillation in the femoral artery. Whereas in the terminal aorta, or in the aortic root the flow is nearly pulse-like (there is a small damped train of waves around zero in the terminal aorta), the flow in the femoral artery oscillates around a mean value. In approximation, the peak is twice the mean flow, and the minimum is onehalf the mean flow. The oscillations in flow are essentially in unison with those in pressure. More to the point, if the flow is integrated, it is found that the volume passing into the femoral artery increases essentially linearly with time, with a moderate oscillation around the line. Then also it appears that the femoral pressure represents an oscillation around a sawtooth.

This modelling begins to represent a different picture of the cycle dynamics than the Womersley proposal of a continuous pulsating cycle, and likely goes back to Frank, or earlier, who likely imagined the cycle decomposed into a variety of segments. In attempting to do this modeling, while it is original with us, and it makes use of many excellent investigators' data, we have had the uncomfortablc feeling that much of this must have been understood - perhaps only intuitively - by the masters in the past. Thus, no claim is made for this modelling, except that of an attempt at physical consistency.

If the cyclic phenomena are washed out of each cycle, i.e. if the 16 cps 'longitudinal' oscillation and $4 \mathrm{cps}$ 'radial' oscillation are averaged, then finally the truer situation emerges. The flow cycle is a pulse, whose integral forms a step. However, there is a leakage out of the system at nearly a constant rate, so that the net difference of input volume and output volume is a triangular wave, which therefore essentially represents the pressure wave. This describes the windkessel. However, what was not obvious before, this also describes the situation at every point in the elastic arterial and venous systems. The system can be viewed as if it had no damp- 
ing, i.e. that there is only little damping that increases the further one gets into small bore tubing. At each level of diameter and volume available there is a sustained moderate sized Muens-Korteweg radial oscillation (i.e. the nearly 4 cps signal) which represents a sustained oscillation around the average pulse and triangular states of flow and pressure; there is also a repetitively excited longitudinal signal (the $16 \mathrm{cps}$ ) excited as the incisura notch when the heart valve slams shut, but this is damped out because Poisson's ratio is near one-half so that there is not much longitudinal motions; and what there is, is prevented by tube tethering. The disturbing thought raised in (2) regarding the question of continued resonaint oscillation is to be answered as follows: The aortic system is resonant, and in constant, sustained resonance. However, the resonance is only radial. Tethering, Poisson's ratio and tissue damping prevent longitudinal motion. There is sufficient damping radially that the damping ratio may be in the $0.2=0.4$ range. (The source is still not so obvious, but it may be achieved by stretching a variety of radial losses - viscosity, visco-elasticity, extraordinary losses because of the particular nature of blood constituents, entrance losses at the many exists, etc. It would take only magnitudes of 0.05 - 0.08 damping ratio factors per source to add up to $0.2-0.3$. )

These explanations add up to a reasonable physically founded model of the hydromechanics of the entire cardiovascular system. As such, it follows the great descriptive beginning by Frank, and sets the stage for a quantitative demonstration of all the events (which may not all be exactly true) both in normal and pathological conditions.

The specific descriptions that must be validated have to be based on the following data shown in Fig. 5. The course taken has been to lay out data, mostly on dogs, around the aortic tree and from its arterial branches from whatever sources have been found available, for both pressure and flow. The sources are identified by reference numbers. Thus in order, the data are drawn from the ascending aortic branch, the abdominal aorta, the descending aorta, and the terminal aorta. Where possible, the time scale and events are identified for the ascending aortic pressure at its beginning rise, the location of the pressure peak, the location of the notch, the end. Flow determinations by a number of investigators out of a few arteries are also shown. When integrated, it is clear that they represent a volume whose efflux increases linearly with time (i.e. the mean flow) with an oscillation around the linear volume efflux. It is clear that the nominal oscillatory frequency at these points is a near $4 \mathrm{cps}$ dicrotic wave and that moderately more damping of the wave is indicated than in the aorta. The proposal is to average these cycles. This is also shown in a second grouping of the same data, but filtered free of these Moens-Korteweg dicrotic waves, in Fig. 5 . Then what emerges is just the constant efflux rate, around which there is an appreciable dampened oscillation.

For example, Wetterer's data which show the flow pulse and volume step from a variety of species are indicated. It appears quite clear that as a result of the cardiac output pulse there is a volume step into the aorta with some sort of ramp function for its front, and that its particular details are not important. During the entire cycle there is a leakage out of the aorta. 
In (2) the leakage was regarded as being a constant efflux out of the entire aortic-arterial system. Here with a little more detail, the efflux out of the aorta may be examined. Flow measurements are not so copious, so that one must accept whatever data are available, much of it commonly out of the femoral artery. However, the efflux from the ' $30^{\prime}$ such stations distributed along the aorta do not represent nearly as much oscillation as, say, at one station. Thus, assuming only a mean flow efflux at each station does not lead to any worse a neglect of oscillation than might be estimated from the oscillation at the femoral artery.

The assumed constant efflux from the arteries subtracts from the ramped step of input flow and creates a triangular wave, as was essentially shown in (2). As developed in (2), this same triangular wave mirrors the pressure in the aorta due to wall elasticity. However, since there was a high frequency notch associated with the valve closing at the top of the triangle, this high frequency must also be filtered out. The two frequencies, the 4 cps 'radial' and 16 cps 'longitudinal' waves have been filtered out in the second grouping in Fig. 5. Thus the pressure waves are shown so filtered at the root of the aorta, in the thoracic aorta, and in the abdominal aorta. The dominant triangular pressure wave shape seems clear. Near the aortic root the dominance of the $16 \mathrm{cps}$ notch and its attenuation both in space and time seem clear. As the aortic terminus is reached the greater dominance of the 4 cps wave appears, so that within the entrance of the arteries, it becomes quite dominant as seen in the figures. However, if this cycle is averaged over, it is clear that the dominant over-all cycle behavior is a triangular pressure wave.

Similarly, inspecting the flow data at all these points, it is clear that the flow data are an early pulse, followed by a constant flow, if 4 and $16 \mathrm{cps}$ waves are filtered. These waves appear as oscillations around the constant (near zero in the aorta) flow part. Again, near the aortic root the 16 cps is dominant, corresponding to the notch, and in the arteries the 4 cps has become more dominant.

The main function of Fig. 5 is to establish, by lumping all the significant data known to us in one place, the primary character of the cardiovascular response. It clearly illustrates the primacy of the elementary windkessel response of a nearly lumped capacitative reaction of the arterial-aortic system with a nearly lumped resistive characteristic associated with the arteriole-capillary bed. While this summarizing idea may not appear complex, it certainly was not found clearly expressed in any of the references, to provide a working quantitative model.

In so doing, it is; perhaps regrettable that this is destructive to the fundamental thesis that was advanced by the Womersley-McDonald-Taylor group that the circulation is to be best understood as an entire system in steady state oscillation, rather than as a system whose behavior consists of a number of coupled transient phases. It is our contention that the system consits of a number of coupled transient phases, and that the oscillatory nature of the system is only of secondary hydrodynamic interest. 
One may agree that these transient phases, of a windkessel nature, represent a repetitive excitation of the oscillatory hydrodynamic elements, but even these are generally of a transient nature superimposed on the 'fundamental' elements of the cycle. The size of these oscillations, as has been suggested, depends on the damping that exists in various parts of the system. The higher frequency component, identified with longitudinal compliance, is fairly quickly damped. The lower frequency, the fundamental radial elastic tube frequency, seems to be little damped in the aorta, as was expected from transmission line theory (2). One would expect the damping to become more appreciable down the arterial system.

This is as far as time permits the problem to be carried at present. The particular nature of the dynamic excitation of the hydrodynamic oscillatory components remains to be accountd for. However, its theoretical foundation has been laid. 


\section{HORMONAL DYNAMICS}

During the preparation of this report, a gross picture of the complex biological system was formed, in which the system consists of a considerable number of autonomous relaxation oscillators (i.e. local 'engine' cycles) whose actions themselves tend to make dynamic adjustments of the homeostatic levels (what was referred to in (1) as dynamic regulators; homeostasis therefore now could be identified better as homeokinesis). Such a description was begun in (1), but it was not clear at the time that it was the main organizing element of homeostasis, for which it is now being proposed. If the concept is true then it would seem to have sume very significant consequences. It is clear that neither (1) nor (2) nor the original proposal on which this study was based was explicit on the action of the hormones, except in very general superficial terms. The weakness, it has been suggested lies in the difficulty of measurement. For this study it was proposed to seek to view the hormones directly in the blood stream, by what would have been essentially an attempt at development of analytic instrumentation techniques. Now, as a consequence of this new view of biological organization, a different and simpler means for showing hormonal dynamics arises as a possibility.

If the hormone, as a 'chemical messenger', is involved in the regulating or control cycle, it can make itself evident as a small signal - requiring amplification - in the regulating or control chain. With a model of autonomous oscillators, the most plausible idea is that it should act to help shift the operating point of the oscillators. In that case, the effect of a hormone should be viewable in its 'transient' effect on the oscillators of the system. That hormones, drugs, chemicals, etco can cause shifts in the oscillators is not new, and it is not proposed as such. That this is 1 ikely to be its basic action is a new thought, as is the thought that the system is tied together as a series of oscillators.

Thus what is basically being proposed here as novel is a complete 'spectroscopy', or 'harmonic analysis', for biological systems. It is proposed that the effect of inputs on the dynamics of the biological system (not the statics) furnishes a new general purpose diagnostic tool for biology. For this present report, an attempt will be made to just scratch the surface. A larger scaled attack particularly for medical effects is outside of the current scope.

The biological system can be viewed in its stable non-linear oscillators (such stable non-linear limit cycles are unstable equilibria), and in its stable equilibria, which upon disturbance, can exhibit transient return to the stable equilibria. These latter states have not been considered in this study which will continue to put them aside at present. To examine various dynamic effects, such as hormonal effects, the following oscillator states may be taken note of. (The ideas and techniques are similar to any other spectroscopic or harmonic analyser scheme in which one may view the excitation of a group of vibrating elements, or networks, or vibrating 'reeds, or spectral lines, in order to explore the dynamics of the system under test. 
The system dynamics may show itself in 'Iine' spectra, 'band' spectra, in line width effects, in intensity effects, in frequency shifting, in frequency jumps such as in fluorescence, in frequency entrainment, etc.).

The salient indicator spectrum that one has available in a complex biological system like man is approximately -
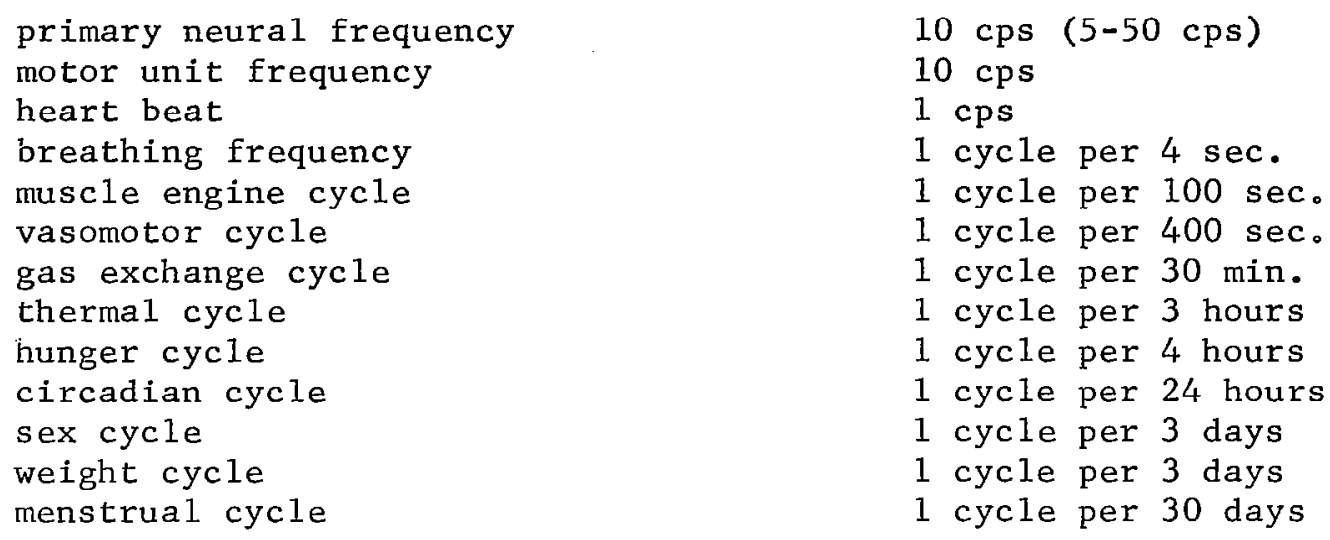

While this 1 ist is perhaps loose, its intent is not. There are data, for example, on rapid cycles at the level of the cell and the small cell chain in nerves that may go up to a few hundred cps. However, it is clear that brain waves, representing a response of the system as a whole (see (1) for example) are quite predominant at near alpha rhythm frequencies (10 cps). It is clear that motor units (see (1) under microvibrations) also show the same dominant frequency. At a slower rate, then the medical doctor and biologist are most commonly aware of heart beat and respiratory frequencies.

Still slower, we have attempted to demonstrate a certain primacy of the 2 minute cycle as a dynamic heat engine cycle. Apparently, knowledge of such cycles is not completely new in biology. A number of biological colleagues have indicated some knowledge of such waves as Lewis waves.It appears that the 7 minute cycle and the 30 minute cycle are also not novel though the 3 hour cycle may be.

As noted in 1926 (55), it appears clear that Lewis, like Krogh, was a very careful observer of capillary blood flow, as he could view it from the surface. It is clear that he began to get a 'feel' for the time domain of blood flow rhythms. While he isn't explicit on the shorter rhythms, evidence points to his awareness of them. For example, he quotes an earlier author "The opening and closing of the capillaries goes on very rapidly so that a drawing made every three minutes shows a different field each time." With regard to step functions of cold or warm, applied to restricted portions of the body, he shows local time constants - under the skin - of 1-2 minutes.

In 1929 (55), Lewis shows some of the nature of the transient response of a small extremity portion of the body, a finger, to cold immersion. The characteristic response is a single overshoot. It is indicated below: 


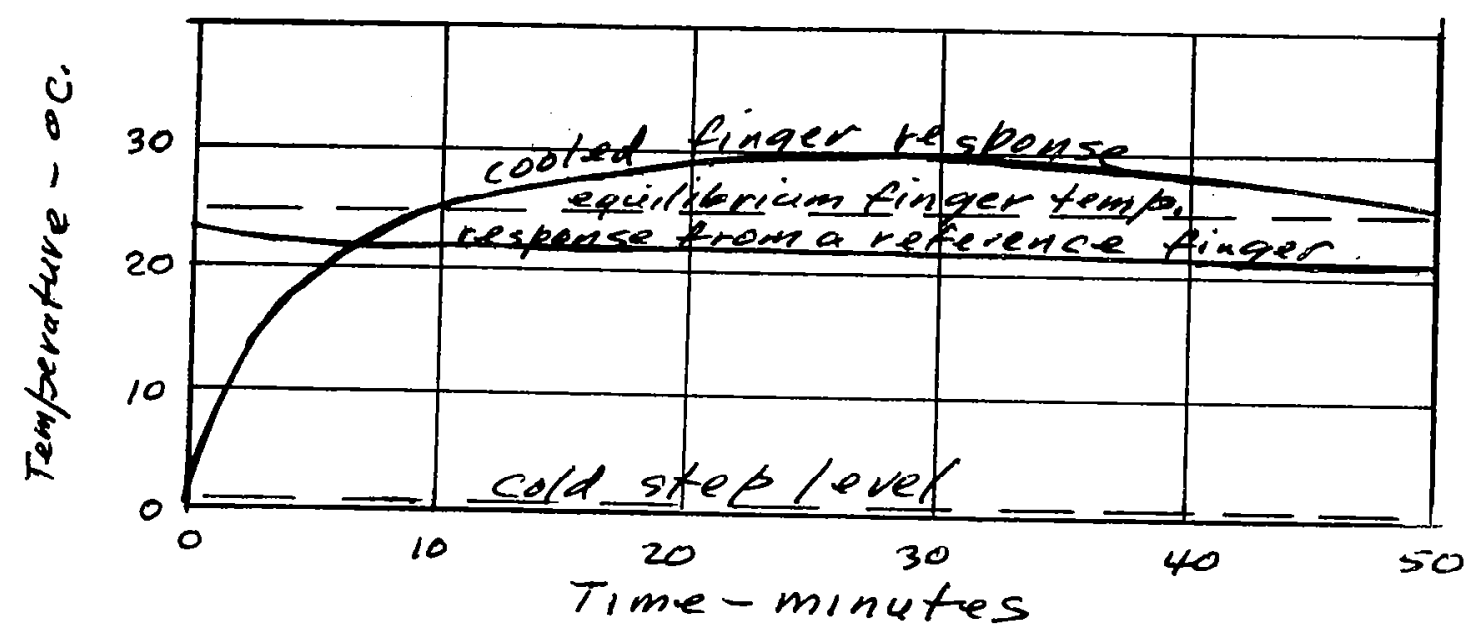

Also weakly evident in a variety of his data is higher frequency cycles of a few minutes duration.

On the other hand with prolonged exposure to cold "it is frequent for the temperature reaction to be repeated over and over again during immersion; this slow 'hunting' of temperature is never quite rhythmic and the rises for the most part are irregular in time and form." He illustrates such responses with data that show 20 minute cycles, and data that show 35 minute cycles much more prominently. Weak 4-7 minute cycles are also evident. A variety of dynamic cycles are shown with the hand immersed in the cold.

One of the curves showing a prominent 35 minute response is reproduced in Newburgh(29). This was disregarded in the past because it had seemed to represent 'artificial' disturbances of the body. Now to view these data against the current systems view begins to be more meaningful.

For example, it is now clear that a point-wise disturbance in space - a finger - or in time - cold immersion for a certain period - can create both transient response and shift limit cycle behavior. The particular prominent 35 minute far-from-regular sinusoids are ample evidence for this. The particular dynamics of the cycle are obviously far from clear. However, one can have little doubt that a cold signal has to be transported centrally, fluid mixing has had to take place, resetting of the blood flow restrictions have had to take place, and somehow a process dynamics slow enough to create a 35 minute limit cycle had to come into existence. If hormones, injected pulse-wise, or repetitiously, etc. do not produce comparable transients, or steady state responses, one would have to consider the entire concept of hormonal action suspect.

A brief summary of Lewis' vasomotor responses to cold may be noted in his 1941 article (55). It is quite clear that Lewis' observations represented an excellent beginning toward a description of vasomotor activity. It is not 
clear that this work has been brought to fruition.

It appears plausible that hunger for food involves a hunger frequency involving a number of eating cycles per day. The circadian cycle is discussed many places at great length. The studies of Kinsey have indicated average sexual incidence of a few experiences per week. Menstrual cycles are again of course well known.

An example of a cycle which is perhaps not so well known is the weight cycle. In (1), the question was raised as to what factor or factors determined the weight regulation, ot how much food intake exists per meal, per day, etc. By accident, a rather rigorous diet was begun at about that time by one of us. As a result, approximately a year's worth of data were acquired, taken nude daily, each morning after urinating and defecating but before eating, on a weighing scale with a sensitivity of about $0.21 b$. and accuracy of about $0.5 \mathrm{lb}$. The food intake wasn't constant for the entire period, but went through segmented changes - a number of months of rigorous dieting, near daily swimming exercise, and periods of other dieting levels。 However, during any number of weeks the eating patterns were fairly consistent. The weight data so obtained are plotted in Fig. 6. It is clear that there is a weight cycle of about 2 pounds amplitude with about a 3 day period. Since the food intake during that period is of comparable rnagnitude, the variation is not due to variation in food weight, but some other factor. It is plausible that the weight variation must be due to a water retention cycle in the body. This is not brought out in the literature, though a significance is always attached to water (such as the thought even that food intake is proportional to water intake). What is illustrated here is that with near constant food intake, the body composition in water content is likely variable cyclical1y by as much as $2-31$ bs.

Now the basic proposal made here is that the action of hormones should sweep across the 'harmonic oscillators' and show their transient presence. That such a thought is not impossible is borne out by the fact that a variety of inputs can involve these oscillators. As a typical example, emotional inputs can sweep the oscillators and 'cause' equivalent physiological changes. (This will be alluded to further in the subsequent section.)

With regard to the hormones, the first experimental objective was to choose those hormone actions that might appear in a short time (i.e. a near high frequency response) such as seconds to minutes or a few hours. (This limitation is just a limitation within the present effort based on keeping the experimental effort low in cost. Equipment becomes elaborate at short times beyond what can be easily viewed within a human response time, or at long times in which elaborate maintainance of the subject becomes necessary. However, the opportunity to inspect the time domain of say 0.5 to 10,000 seconds, i.e. covering 4 decades of time, is not a trivial effort). Since it is conventionally regarded that there is considerable control action exercised by many hormones in this time domain, it could prove to be a fruitful testing ground for these theories.

The purpose of these experiments was to contribute background information toward a detailed picture of the dynamic response of the system. 
For the desired domain, it was proposed to use two oscillators as indicators - the heart rate and the ventilation rate (i.e. the metabolic rate, per Gray) averaged over individual breaths.

It is useful to have some idea of the normal records. Fig. 7 shows some preliminary data on a human subject. First, the ventilation rate (i.e. minute volume,) again reveals clearly a near two minute cycle, regardless of subject. Second, somewhat a little surprising, the heart rate also shows a similar cycle.

The minute volume was determined by breathing from a demand regulator supplied by a fixed high pressure container which was intermittently recharged. The minute volume was obtained from knowledge of the pressurevolume characteristics of the container. The heart rate was obtained from a microphone laid over the heart and supplying pulses to an electronic counter which had been set to count the time for numbers of cycles. Ten cycles, in particular, proved useful. Alternately, the input was also obtained from electrical leads supplying signal. The signal was highly amplified and filtered to avoid extraneous noise, and is reasonably clean though not absolutely free of spurious signal.

Inspection of the data shows the following cycles:

1. A near two minute cycle in ventilation rate;

2. Approximate 1 1/2-3 minute cycles and 7 minute cycles in heart rate;

3. A $11 / 2$ minute and a 7 minute cycle in finger temperature;

4. Rises in mean finger temperature, and ventilation rate with the drug isuprel. In some experiments there was an indication of a rise in heart beat rate with isuprel。

The resolution possible above noise level, by the techniques employed, permit identification of perhaps up to 5,000 second cycles, and perhaps down to 30 second cycles. Thus, although a 5000-10,000 to 1 band is explored, quantitative response can only be obtained over 2 decades (100-200 to 1). Such drastic limitation is not uncommon in spectroscopy.

A few such casual experiments with 'chemical' upsets, such as oral carbonate disturbance, or a cardiac stimulant and vasodilatant (isuprel) were investigated. It became quite clear that the entire thesis of hormonal dynamics had to be studied with regard to direct additions to the blood. It was therefore much simpler to begin to experiment with another animal, which very soon led to the choice of a dog. Apparatus was quickly adopted to measure ventilation rate and heart rate for a dog.

The effects of a variety of hormonal actions on the dog are illustrated in Fig. 8. The drugs used in the experimental sequence wcre: 
sodium bicarbonate

adrenalin

insulin (NPH) $\mathrm{U}-80$

hydrocortisone

vasopressin

$\mathrm{ACTH}$

parathyroid

thyroxin

acetylcholine
$0.5 \mathrm{gram}$ in $5 \mathrm{ml}$ of water

$2 \mathrm{ml}$ of $1: 10000$ adrenalin chloride solution

$0.1 \mathrm{ml}$ of $\mathrm{U}-80$ in $1 \mathrm{ml}$ of water

$10 \mathrm{mg}$ in $1 \mathrm{ml}$ of water

0.5 USP units in $2 \mathrm{ml}$ of water

5 USP units of Acthar gel in 2 $\mathrm{ml}$ of saline solution

5 USP units in $1 \mathrm{ml}$ of water

$5 \mathrm{mg}$ in $4 \mathrm{ml}$ of water

$50 \mathrm{mg}$ in $1 \mathrm{ml}$ of water

For general references on dosages and effects, Salter (56), and Goodman and Gilman (57) were used as source books.

Each day, an $8 \mathrm{Kg}$. dog was fitted with a mask, and a microphone placed over his heart. He was then confined in a wooden kennel with his head sticking out, and with a shield hung over his chest so that he could not scratch off the mask or microphone. While generally confined, he could rise and lay down.

Data were taken on the ventilation rate in segments, with a skipped period between segments to permit temperature equalization in the supply gas cylinder. Determinations were made every 10 seconds, and the minute volume was determined for each 10 seconds interval during the total period of about 5 hours cach day. These data are plotted in Fig. 8 for each of the test periods. In each case, the first few hours of data were taken prior to injection of the drug directly into the blood stream (in the cephalic vein). Data taking was recommended as soon as practical, after calming the dog down and reattaching the equipment. The procedures were not perfectly satisfactory or complete, but represent a first preliminary attempt.

Heart data were taken as the counted time for 10 beats (i.e. nominally 5 to 10 seconds), and a similar time skipped for computation by the counter. These data were recorded by plotting the count time against serial clock time. Since the rate is given essentially directly by the counter, it was not necessary (nor possible) to note the time of counting closer than a second or two.

Al1 data, both noisy and not, were recorded. Noise would commonly develop when the dog moved around. In presenting the heart data in Fig. 8, all the data that were considered noisy because of movement were cut out, in an attempt to represent more nearly only heart rate. While a fair attempt was made to derive an accurate picture, it is not possible to certify, without reservation, the complete validity of these heart data.

The ventilation and heart data reveal the following:

1. There is an approximate 1 minute cycle in ventilation rate in the resting animal. 
2. A similar cycle is present in the heart rate.

3. Of the hormones and chemical tested, only adrenalin and acetylcholine showed noticeable effects. Acetylcholine produced an immediate physiological effect, the animal vomited and was shaky and upset. By the time he recovered and data taking started again, ventilation and heart rate had settled back to normal. Similarly, with adrenalin, though there was no very obvious system upsets; when data taking was resumed several minutes after administration of the hormone, the heart rate was on the way down to normal from an apparent elevated state.

4. The use of NPH insulin was not a wise choice for effects in the time range, because this insulin has a longer time delay than regular insuIin.

5. The other test substances did not show apparent effect on ventilation or heart rate, presumably because their action may be out of the time range, or they may be specific for other effects than those that were monitored, or larger doses are required. (For example, it is noted (57) that whereas small doses of vasopressin affect kidney action, much larger doses are required for increasing blood pressure and the heart rate. The same reference (57) states "there is no reason to believe that the pressor action represents a hormonal function; rather it is an incidental, and parenthetically a rather useless pharmacological property of a highly specific hormone.")

The data thus far taken represent a considerable mass of fairly precise data that indicate the common operating dynamics of two body oscillators. The action of some test hormones in sweeping across these oscillators was examined, with preliminary results that are still somewhat negative. However, the concept of determining the hormone dynamics by their transient effects on the system oscillators is still considered valid. What are required in subsequent experiments are conditions which will permit more specific attention to a variety of details, such as more rapid return to data taking after administration of the hormones, so that the short time transients may be noted. It will also be necessary to review the dosages and more specific action of hormones for ones which will be more likely to have effects in the time range and on the particular system oscillators being studied.

With this beginning there is confidence that an experimental program can develop to yield interesting and valuable information on the dynamics of some of the hormone actions, taken on intact animals with little or not interference in the normal body activities. 


\section{BEHAVIORAL ORGANIZATION}

\section{A. Speculative}

This section will continue to be a collection of a wide variety of ideas, in a continuing attempt to sort out a coherent picture of behavioral organization and dynamics. The frame of reference will continue to be, not amateur psychologist, but professional physicist. However, what is being sought are those ideas that seem to be useful physically to put together a system model. It appears that this was the aim of such programs as the bionics program of the Air Force, or earlier attempts by Norbert Wiener in cybernetics, or partially, in the theories of automata, etc. The psychologistpsychiatrist may have to grit his teeth while kindergarten is conducted. In this first portion, a sequence of speculative ideas will be discussed that have been considered since the last report.

An overall physical modeling of the biological system, say, in man or other of the less primitive species, may be of a cylindrical food tube dedicated to the task of taking in food at one end, distributing product and by-product along the tube and orthogonal to the tube, and excreting wastes at the other end.

The need for acquiring food is accompanied by a capability for motion. Thus a variety of complex carriage mechanisms are wrapped around the food tube. However, this raises the fundamental question of how the driving mechanism to acquire food is organized. The broad concept of the pleasurepain principle, given form by Freud, appears to be at the root of the system self-activation that is required for accomplishment of a considerable number of primary duties of the system.

For example, it appears that there are very primary erogenous zones at both ends of the tube, associated with Freud's anal and oral eroticism. At present, the food drive and the sex drive are mixed up in our mind. Frankly, at this time, the anal zone functions are not understood by us at all. (It has not helped physically to discuss this with psychologists, who seem to give a social interpretation to the significance of anal croticism. It would seem that Freud was proposing, and with possible validity, something more fundamenta1.)

The following somewhat literary summary was written to express the problem ideas more forcefully.

\section{The Two Gluttonies}

Though we were brought up in middle class morality, it was in a generation in which the verbal concepts of sex and other free intellectual inquiry were freely discussed and talk of Freud filled the air. So that the reader doesn't misunderstand, basic Victorian prudishness about the subject, however, still was left in that 
generation, even if the verbal freedom was considerable. Yet a basic scientific point was never brought to our attention, with all the talk of Freud, until now. Humans, and likely most biological systems, are basically driven by two gluttonies - the gluttony of sex, and the gluttony of food. Both of these are 'hungers' though the scientific case is obviously overstated, or at least simplified, Other hungers represent significant drives too, and are quite basic. But of all so-called 'volitional' activities, it has emerged, amazingly, how powerful and polarizing is a 'gluttonous' and'ravenous' turn toward food and sex in the human animal in all circumstances, even more obviously, when these matters are not matters of any sharp unsatiated or unrequited hunger. Any group of males-females will have to speak for themselveswho have found themselves trapped in a somewhat sexy situation, individually or in a group, know how lustfully, and quickly and shamelessly the entire group attention is involved. One must simply note male attention reaction when the slightest sexually suggestive act of a female flickers across their common attention. The repetitiousness of it is spelled out in generation after generation of literary endeavor. In most boring detail it is spelled out in Henry Miller's two "Tropics", or Casanova's exploits, etc. One can also note the gluttony in food in any house gathering an hour after dinner; in any pleasure resort where people's needs are served; in older people as their inhibitions become lost; or in younger people whose social inhibitions haven't yet developed. Other hungers are periodic, and are recognized as involuntary. The case being made here is that two apparently voluntary hungers are not voluntary and are also likely cyclic and organized in primary oscillator fashion.

From this emerges a first model of the viable, self-locomotory biological system. In (1), it was described as a bathtub of chemicals that were chemically regulated as a homeostatic, i。e. regulated system, with a considerable number of oscillators tossed into the bathtub, and with the dynamic stability, i.e. dynamic operating point in frequency, amplitude, and detailed topological phase plane characteristics, chemically mediated. In this report, there has been a very basic shift in view. The oscillators themselves are the determinants of homeostasis, $i$. e. according to a dynamic regulation scheme using the concepts of (1). However, it is not yet known what the built-in algorithm is by which these oscillators determine and adjust the mean levels, and how the coupling is arranged to change the oscillators to readjust the homeostatic levels. AlI such elements of the story must emerge slowly.

However, in the present concern, the problem is how to get the system moving. In the first report, a great deal of discussion was always punctuated by the idea of the self-actuated system. Now, it is finally proposed that the system might be best described as an elongated food tube in and along which food can be taken in, processed, and eliminated, and in which the elongated form will allow sufficient process lag time, and which will permit the bathtub of 
chemicals to be wrapped around the tube. Conversely, one views the tube as being immersed and passing through the bathtub. At each end of the tube, there are erogenous zones, one food seeking and the other sex seeking. There is no implication of lack of interchangeability of interest or excitation; and certainly none that a very pat one-two solution is being sought. It appears to be simply a statement of fact that both ends are endowed. Why not only one end is certainly not clear at present, and why there should be an anal as well as an oral and a sexual eroticism is certainly not clear. The totality of these proposed ideas are obviously far from original, and are not intended so. However, the geometric organization becomes an interesting first physical idea at this point in the development of the argument. The total effect of these two major hunger drives is to propel the organism to a general meandering in search of satisfaction.

U1timately one must accept a bloody raw core of biological gluttony if one wants to make progress in decoding the biological system.

One may also note through all of this discussion that one is forced to a tripartite argument and view simultaneously of the description of physical systems, of the biological system, and continued underlying implications for the dynamics of the social system.

While in poetic description the drives were referred to as gluttonies, it is likely better to describe them so as lusts.

Reading C. Wright Mills' THE POWER ELITE, it has suddenly dawned on us that one must add two more major lusts - for admiration and for power. As one feels the impact not only of Mills' description of current society, but of societies through all of man's rccorded history, it comes out in a purely impersonal way that humans crave admiration and crave power in ways that are just as powerful as for food and sex. Once again, these cravings are not for an elementary physiological satiety, but the human can become gluttonous in his desires.

Thus these large scale 'behavioral' mechanisms, which are proposed, are oscillator controllcd regulators, relaxation oscillators, making up part of the growing picture of hungers that the biological system has. These now include (as major ones) hunger for:

Oxygen

Attention

Water

Activity

Sensory stimulation

Food
Sex

Anger

Thinking

Admiration

Power

The list is not exhaustive nor even sharply devisive. However, it is proposed 
as a tentative scaffolding which may change as needed. While it is proposed that each of these are tied up with an oscillator, so that there is a priinary frequency associated, the triggering capability of phase adjusting these relaxation oscillators, using the cast of the individual's pleasurepain mechanisms, can vary the individual's lusts.

A few remarks on these are in order. The pairs -

Oxygen - no oxygen

Water - no water

Food - no food

are quite obvious as extrenes of the amplitude excursions exhibited by these hungers. One nay also note another characteristic, that the inputs and outputs may be separate and may involve storage elements, etc., i.e. an entire complex of mechanisms, and oscillator cycles may be tied up with each. Under this circumstance, the following pairs may also be understood,

Activity - no activity or rest. The rest-wake cycle, the activity cycle varying with menstrual period, the motor unit cycle, the seasonal cycles, etc. all illustrate the fact that an enforced state of activity or no activity cannot be well accepted for long periods of time.

Sensory stimulation - no sensory input. Sinilarly, the organism cannot shut out sensory input without vertigo, disorientation, irritation, hallucination, etc.

Attention - non-attention. This is of an internal variety. Very likely almost fundamental is the biological system's self-awareness which would appear to be cyclic. variety.

Admiration - disregard or abhoration. This is an external seeking

Power aygressive - non-aggressive. The latter two emerge as cornerstones of bellavior, and represent at least one well known break with Freud.

Anger - tranquility. It is likely that this is a hunger, involving like food, etc., a periodic discharge of emotional wastes to equalize the system.

Thinking - no thinking. This is also 1 ikely an intermittent activity to discharge 'tensions' internally in the system.

One notes these hungers occur in pairs, and also considerably as poles of behavior. People are aggressive or non-aggressive, gluttons or diffident eaters, sexully active or passive, thinkers or non-thinkers, etc.

In this very crude first model, it emerges that it is a complex of possibly 'centers' for pleasure-pain which represents the individual's behavioral computer coding, and represents how he pushes the buttons (i.e., 
determines the 'cortical' algorithm) for behavioral controls of these oscillators.

(For a crude illustration, one author was overweight and wheezing, and had particular behavioral breathing patterns to adapt to short breath, etc. He lost weight and took up swimming. As adaption to swimming, which forces a change in breathing pattern, it is certain that a much slower and more uniform breathing pattern has developed. The original adaptions had to be 'cortical'. Most beginning swimmers fight breathing problems. Gradually this must be shifted to a 'hypothalamic' routine. Similar or related stories could be told with regard to food patterns, sex patterns, and even admiration and power patterns. Another crude illustration may be cited in the latter categories. Having four children has involved emotional problems,particularly with an extremely strong-minded father. At least one child warranted some attention to the extent of using outside guidance. Two years with a family guidance clinic was most helpful. However, in this program the father had considerable to learn. One trait was an over-intellectualization of problems. The subject was considered quite capable of seeing most other problems, and perhaps being able to reduce them to intellectual terms, but he could not see his own. The basic problem was a constant probing at other people to test whether he would be rejectled. It is not straining at imagination to suggest in retrospect, and even at present, that there is a considerable amount of periodicity in this probing and testing even if not demonstrated quantitatively. The point here is not to review any amateur psychoanalysis but that most bystanders are conscious of the on and off churning of enotional drives in people whose purpose is not inmediately clear, and which to some extent the human can switch on and off.)

It is hardly fair yet to consider these coding drives to be associated solely with very simple two state pleasure-pain centers, but the organization scheme of the system is helped by starting off with such simple view.

One final piece of speculation emerges which may also be useful. In the first report (1) and elsewhere, we resisted regarding rapid biological responses as being transit time limited. It is obvious that there is a transit time. (Just as in a house electrical system, one might say that there is a transit time governed by the velocity of light. At $3 \times 10^{10} \mathrm{~cm} / \mathrm{sec}$, it is clear that in a $1000 \mathrm{~cm}$ house, the 0.03 microseconds of transit time does not govern very much of the ordinary electromechanical responses of equipment in the house. However, if there are other phenomena that are molecularly governed, then the velocity of propagation of sound waves $-30,000 \mathrm{~cm} / \mathrm{sec}$. in air, and $300,000 \mathrm{~cm} / \mathrm{sec}$. in solids, or 0.03 seconds time delay in air - can begin to make itself evident in some mechanical phenomena in the house.) It is obvious that the transit time is not overly fast, typically numbers of the order of 100 meters per second (for very fast nerve transmission) or 0.02 0.03 second transit times are given. The elementary impression is left that elementary reflexes of the order of $0.1-0.15-0.2$ seconds in humans are made up of conduction times at synapses plus transit times along the nerve. 
However, considerable issue was made in (1) that a maximum frequency of the order of $10 \mathrm{cps}$ for reflex complexes involving training seemed to exist. The conclusion was resisted that this maximum frequency is determined by these transit times. What is being sought is the logic of the organization. One may argue that the same characteristic limit of near $10 \mathrm{cps}$ exists in small animals, and is thus not an intrinsic size scaling law. Thus some other idea would be useful. The following is proposed.

Suppose a response provoking input is presented to the biological system. The system responds, much as a telephone system, in which autonatic and nonautomatic switching networks are involved. This response will involve some time delay. If the system is called upon repetitively to perform the same task, the 'cortex' may find shorter time paths involving more autonatic switching. With repeated efforts, the cortex likely transforms almost the entire pathway response to automatic arrival time switching, and likely also records the entire process in memory. It is proposed that this represents a primitive model of the conditioned reflex and a primitive model of why much of cortical operation can continue to appear to operate with near random switching network properties. As time goes on the cortex may re-examine the problem and find even shorter time pathways (or less energy pathways). The basic idea that is here cast is that the lumped elements out of which the electro-chemical-mechanical response is made up are basic oscillators in the vicinity of $10 \mathrm{cps}$. The overall response of the system is time governed not by transit time, which may govern some of these $10 \mathrm{cps}$ oscillators - but by the number of network oscillators that have to be connected. This means that the system as a whole is likely to be treated as luriped networks, rather than as distributed networks. The unanswered question still remains, what constitutes the individual high frequency uscillators?

Having touched on these rudimentary attenpts at a general speculative system outline, the effort may continue toward more review.

That the central thought of oscillator organization - even for behavioral elements - is plausible may be seen in such a book as THE MANIPULATION OF HUMAN BEHAVIOR, Biderman and Zimmer, H. (58), which shows, in one section, the section on Physiologic Responses, that some physiological concomitants of emotional disturbances on physiological cyclic paranctcrs are well known, and measurable and even standardized in a number of cases. It is far from accidental that many are the same ones listed in this study. 


\title{
B. Review and Commentary Continued
}

Continuing the discussion in (2), further material will be reviewed. First Freud nay be further viewed in Jones (59.) from 1900-1920. In recapitulation, Freud had worked in his $20^{\prime} \mathrm{s}$ in neuro-physiology and was thus quite conversant with neuro-anatomy; he had then worked in clinical. practice in neurology, with a practice devoted largely to neurotics. Starting with hypnosis, he picked up the cathartic method of treatment of Breuer's, and developed the concept of the sexual causation of neurotic disturbance. His studies compelled him to the importance of sexuality in early childhood, and its incestuous nature. He developed the ideas of distortion mechanisms which repress forbidden impulses from appearing in consciousness. Finally he developed a theory of dreams. In these latter efforts he left neurology and began the field of psychiatry.

The Psychological Wednesday Society, later the Vienna Psychoanalytic Society, began in about 1902, with Freud, Adler, Kahane, Reitler and Steke1. Later others joined, some to become wel1 known, such as Graf, Federn, Hitschmann, Rank, Ferenczi, and others. The period 1900-1906 also saw the emergence of writings by others in psychoanalytic theory.

In 1904 Freud wrote THE PSYCHOPATHOLOGY OF EVERYDAY LIFE, possibly his best known book among the general public. In 1905 he wrote the second of his most important books, THREE ESSAYS ON THE THEORY OF SEXUALITY (with THE INTERPRETATION OF DREAMS).

Dr. Bleuler, Professor of Psychiatry at Zurich, and his staff had been occupied with psychoanalysis from about 1902, and by 1907 his chief assistant, C. Jung, published his history-tiaking book in psychiatry, THE PSYCHOLOGY OF DEMENTIA PRAECOX. (Freud and Jung were on friendly terms for about 7 years, 1906-1913. Jung's admiration for Freud did not extend to his followers.)

A measure of the international recognition that had already accrued to Freud by 1008 can be noted in the first public and international recognition of Freud's work in Salzburg, in a Meeting for Freudian Psychology. The attendees represented quite a sampling of the early important workers in the field. The paper titles are illustrative of the problems of concern then.

\author{
Freud: "Case History" \\ Jones: "Rationalization in Everyday Life" \\ Riklin: "Sone Problems of Myth Interpretation" \\ Abraham: "The Psychosexual Differences Between \\ Hysteria and Dementia Praecox" \\ Sadger: "The Aetiology of Homosexuality" \\ Steke1: "On Anxiety Hysteria" \\ Jung: "On Dementia Praecox" \\ Adler: "Sadism in Life and in Neurosis" \\ Ferenczi:"Psychoanalysis and Pedagogy"
}


In 1908 with Jung and Bleuler, Freud helped found the Jahrbuch fur Psychoanalytische und Psychopathologische Forschungen. One of Freud's 1908 papers, a short paper, laid out the theory that anal sensations in infancy - anal eroticism - affected character traits in very specific ways.

Freud visited America in 1909 with Jung and Ferenczi, invited by Stanley Hall of Clark University. His American disciple was Brill (and Jones in Canada). Morton Prince had opened the Journal of Abnormal Psychology to Freud. Others who befriended Freud in Amcrica were J. Putnam, Professor of Neurology at Harvard. (It is quite clear that a cult had already formed around Freud by this time.)

By 1910 the psychoanalytic movement was launched. For example, the Salzburg Congress became the first of 18 such meetings (through 1954). The second, technically fruitful, personality-wise stormy, congress was held in 1910 at Nuremberg. (One interesting technical bit at that meeting was an effort to start a collective study of symbolism). Apparently both a base for psychoanalysists was laid, and the rift begun which drove Adler and Jung off in other directions. Under Adler and Stekel a Viennese journal, Zentralblatt fur Psychoanalyse, was begun in 1910. Branch societies of the International Association which had been formed were begun, for example, one in Berlin with Abraham as president, one in Vienna with Adler as president, one in Zurich with Binswanger and Jung. By this time psychoanalysis was widely discussed at varlous medical meetings in Europe, but seldom favorably. American acceptance was more favorable, material being accepted in the Journal of Abnormal Psychology and the American Journal of Psychology in this period of about 1910. Bril1 had already begun translations in 1909. In 1910, after the meeting of the American Neurological Association, Jones helped get the American Psychopathological Association started under Prince as president.

Early days of Russian interest involved the names of Ossipow, Wulff, Wirubow, Drosnes, and even the appearance of a periodical, Psychotherapia, with psychoanalytic papers in 1909. The interest was short-lived.

These few paragraphs illustrate the launching of psychoanalysis and the beginning of its spread from about 1910. As a measure of its content at that time Freud's FIVE LECTURES ON PSYCHO-ANALYSIS, published in 1910, is quite useful. It includes his American and Nuremberg papers. One may note, for example, that by 1911 Freud expressed the thought that his originality was unmistakably vanishing.

In 1911, Bril1 started the New York Society, and Jones the American Psychoanalytic Association. The interest in the subject can be noted by the fact that the 1911 Harvey Lecture by Putnam was an invited one on the subject of psychoanalysis.

In 1911, Adler broke with Freud. In 1913, Jung broke with Freud. Clearly, the psychoanalytic movement had become a 'religion' by 1913. (Freud's remarks, quoted in Jones, p. 104, is fair evidence. Thus one must certainly be careful about accepting the objectivity of material from this period on.) 
Notable in this period before World War I was the violence of opposition to Freud, very little of it in print. Discussion involving sex bore a stigma of evil-mindedness, and wickedness, and disrepute. Freud and his. followers were considered perverts and psychopaths. (All of this makes it very difficult to draw objective judgments from the period.)

The problem of dissensions is particularly difficult for psychoanalysis for the following reason. "Investigation of the unconscious, which is a fair definition of psychoanalysis, can be carried out only by overcoming the 'resistances' which ample experience has shown are displayed against such a procedure. In fact, as Freud has remarked, psychoanalysis consists in an examination of those resistances and of the 'transferences' that accompany them, and little else. When the resistances have been overcome the subject has insight into aspects of his personality to which he had previously been blind."

(This summary was so specific we felt compelled to quote it so as to avoid accusations: of not understanding the rudiments of psychoanalytic theory. However, a physicist must continue to point out that such descriptions literally point to the nonlinear nature of the human behavioral system, and to the idea of synchronization, which is purely a non-linear idea。

In scientific objectivity, one would expect to be able to avoid personalities. However, it is impossible to read in this subject, or to have been engaged in scientific originality, without knowing that the nature of the bias, the vested interest, the attachment, etc., all Freudian-like concepts, cannot be avoided.)

Many of the dissenters were partisans, whose initial resistances had been overcome, who had undergone analysis, but their views or acceptance did not stay firm. "The forces in the mind are not static but dynamic. They can vary and shift in unexpected fashion".

(Again it must be pointed out, as in the previous speculative section, that the compelling nature of cyclic phenomena - without apparent personal causality - is part of the nature of these nonlinear limit cycle oscillators.)

The divergences from psa (psychoanalysis) have had two features: repudiation of the essential tenets of psa, and exposition of a different theory of mind.

(It would appear fairly clear that one cannot repudiate the essential tenets of psa, and yet one must go on to a different, or at least more complex, theory of the mind. Thus any permanent partisan on either side, either for or against Freud, loses claim to objectivity. We have a similar reaction to say Halberg and circadian rhythms, or Wiener and cybernetics, or closer to home, Newton and classical physics, or Einstein and relativity, etc. One must always feel that their 'slips' are showing by their partisanship. However, 
this is not necessarily bad for a pleader for a cause. It's just bad for the overall objectivity.)

According to Jones, an unpleasant, backbiting, quarrelsome state developed between the Viennese group under Adler and Freud as an image of the personality side. On the technical side, according to Jones, Adler's views become essentially one of the psychology of the ego, neglecting the manipulation and influence of the unconscious processes. Adler's view of the ego, according to Freud, was like that of a circus clown who claims to accomplish all of the difficult circus feats. Adler's basic tenets involved a tendency of the system to overcome feelings of inferiority by an innate aggressiveness. At first Adler viewed this as a broad sexualistic view of conflict of masculine and feminine components. Later he interpreted things in terms of a will to power, even in the case of sexual intercourse. Adler discussed his ideas at considerable length at the Society meetings in January and February 1911. In what has become known as deviational excommunications of the far left and far right, Freud stated, "I feel the Adlerian teachings are incorrect and therefore dangerous for the future development of psychoanalysis. They are scientific errors due to false methods; still they are honorable errors. Although one rejects the contents of Adler's views one can recognize their consistency and significance."

(Fifty odd years later, one can see nothing wrong with both views. In fact in the previous speculative section, it was left that sex, admiration, power all seem to be hungers or 'lusts' with cyclic complexes tied around each. Whether one is basic or all three are interrelated, or not even unique, is not completely the point. The clue can be taken from the previous sections on the heart and hormonal system, and general system dynamics. In a complex spectrum of effects, each element arising on its own time scale, the interconnection between elements in one complex or another are not obvious, and yet that is how they arise. A two minute power cycle shows itself in the heart rate. The four second breathing cycle shows itself in the heart rate. The eating cycle shows itself in the heart rate. The menstrual cycle shows itself in the temperature cycle. Emotional disturbances show themselves in all physiological oscillators, ad infinitum. The basic scientific questions stem around how much of the sexual dynamics, whether cyclic or otherwise - but in various time scales have Freud and his followers managed to dissect? Has Adler identified another independent dynamic complex in aggressive behavior, etc.? It is these questions, always from the point of view of the time domain and the pole of behavior, that a physical scientist must continue to bring to the problem. The biological reader will not be impressed at first, because the elements assembled will always appear to be trivia1. They are not trivial; they are primi tive. However, the pile of evidence will be made to grow in each instance, until a picture of the complex begins to emerge.)

Adler and Stekel resigned. 
(In support of such a simple view of the issue as proposed here, there is a summary of Freud's, on hearing that Adler had been invited to America to lecture, who said, "Presumably the object is to save the world from sexuality and base it on aggression.")

While. Adler was regarded as superficial ("He simply ignored the methods and findings of psa'), Jung's break was more serious. According to Jones a major issue again was the emphasis on sexuality as a base. A Swiss could not buck the prevailing moral code in Switzerland because of its closeknittedness. Thus practically every Swiss analyst had to abandon Freud's sexual theories.

(It might be noted that Freud attempted always to drive his followers back to the neuroses, and not let them stray into any social issues.)

Personally also Jung became more interested in his studies in mythology than in his presidential affairs with the Association.

(Jung's technical idea was that the fear of an impulse to incest was the key to mythology.)

In 1911 and 1912, Jung's essay on Symbols of the Libido, in its second part began his serious divergence from Freud. Generally, the divergence involved Jung's emphasis on an incest complex.

Thus by 1914 the split in 'psychoanalysis' in the directions of Freud, Adler and Jung had been accomplished.

(However, one must agree with Freud's comment that "Anyone who promises to mankind liberation from hardship of sex will be hailed as a hero..."')

Though its sexual motif certainly has created discomfort even among scientific people, it is hard to overlook the basic core that Freud laid down.

It is useful to note that of his followers, Freud thought most highly of Kar1 Abraham's intellectual powers, although Ferenczi stood closes to him.

(It is to be noted that the Freudian movement as of 1914 was a1so insulated and identified as a "Jewish" movement; its central "defense" committee for Freud at that time consisting of Ferenczi, Abraham, Rank, Sachs and Eitingon, with one adopted "outsider", Jones.)

Freud's scientific detachment did not prevent his taking strong nationalistic views favorable to the Central Powers during World War I. 
Typical of Freud's scientific thinking at that time is an abstract from a letter date in 1914.

"The only satisfactory thing going on is my work, which is in fact leading, despite recurrent pauses, to noteworthy new ideas and conclusions. Recently I succeeded in defining a characteristic of the two systems Bw (consciousness) and Ubw (the unconscious) which almost makes both of them comprehensible and which yields what I think is a simple solution of the relation of dementia praecox to reality. Al1 cathexes 1 of objects make up the unconscious. The system Bw signifies the connecting of these unconscious ideas with concepts of words: it is this that gives the possibility of something becoming conscious. The repression in the transference neuroses consists of withdrawing libido from the system Bw, i.e. in separating the ideas of objects and words. In the narcissistic neuroses the repression withdraws libido from the unconscious ideas of objects, naturally a much more profound disturbance. Hence the changes in speech in dementia praecox, which in general treats the ideas of words as hysteria does that of objects, i.e. it subordinates them to the 'primary process' with its condensations, displacements and discharge. I could now write a complete treatise on the theory of neuroses with chapters on the fate of instincts, on repression and on the unconscious if only the pleasure in working were not disturbed by my bad mood."

(It will become increasingly clear that Freud was taking a point of view toward the interior that is most reminiscent of people's concern in the past 10 years with the problem of machine translation and searching of information. Much of the problem is a search for a basis for the intermediate 'machine' language. The characterization of this problem in the human is one of the major contributions that Freud made more than a half century ago.)

Another significant thought that Freud made explicitly, at about this time, is contained in a letter in 1915.

"The unworthiness of human beings, even of analysts, has always made a deep impression on me, but why should analyzed people be altogether better than others? Analysis makes for unity but not necessarily for goodness. I do not agree with Socrates and Putnam that all our faults arise from confusion and ignorance. I think that too heavy a burden is laid on analysis when one asks of it that it should be able to realize every precious idea."

(It is quite clear that scientific understanding and morality are not associated, which thus contains another implication that any well operated state of the human system in itself contains no premises of morality. One must infer from this that the decoding of

1 These may be defined as a distribution of object images in memory space. 
the human must only involve mechanistic elements divorced of value and moral judgments and idea1s.)

Two interesting comments, one from 1914 and one from 1915, are that he had "solved" to his satisfaction the psychological problem of space and time (Nov. 1914 in a letter to Ferenczi), and a second that he had obtained insight into the primal basis of infantile sexuality.

In 1915, Freud also undertook a synthesis of his conceptions in 12 essays, TOWARD AN INTRODUCTION TO METAPSYCHOLOGY. He apparently destroyed 7, so that only 5 remain. They were on instincts, repression, the unconscious, a supplement to the theory of dreams, and on melancholia (an introduction to his later theory of a death instinct).

While the war years in general were marked by little effort in psa and a considerable degree of depression on Freud, one 'practical' result was that the considerable appearance of 'war neuroses' and the efforts of Abraham, Eitingon and Ferenczi made an impression on some higher ranking army medical officers and some thought was given to the erection of psa clinics.

The state of development may be noted in Freud's paper on "Lines of Advance in Psycho-Analytic Therapy" at the 5 th International Psycho-Analytic Congress in Budapest in 1918.

"Despite the extreme shortage of printing paper and type Freud succeeded in publishing in 1918 the fourth volume of his Sammlung Kleiner Schriften; with its 717 pages it equalled al1 the three previous ones put together. It contained two long papers that had not appeared elsewhere. One was the extremely important "History of an Infantile Neurosis," one of his series of long case histories. The other, "The Taboo of Virginity," was a continuation of his anthropological studies initiated by Totem and Taboo. We shall come back to these later.

"Like the previous two this was a fallow year for Freud so far as original ideas are concerned. There was no hint of the coming recrudescence that startled us all in the following year. Periodicity was of Freud's nature, as of that of most original thinkers."

Jones then proceeds to review Freud's works. In this period, 1900-1920, Freud's production was voluminous, mostly devoted to working out the earlier, fundamental theories. The libido theory was amplified and widely applied beyond clinical problems.

Expository examples from this period include a little book ON DRFAMS (1901); an essay on "Mental Treatment" (1905); partial1y expository COLLECTION OF SMALL WORKS (5 volumes, respectively 1906, 1909, 1913, 1918, 1921); very popular were FIVE LECTURES ON PSYCHO-ANALYSIS (1909), the substance of his American lectures; a 1911 paper for the Australasian Congress in Sydney; a 1912 essay "The Claims of Psycho-Analysis to Scientific Interest", a fairly comprehensive summary of the relation of psa and other 
branches of science; finally Freud's most popular book, INTRODUCTORY LECTURES ON PSYCHOANALYSIS (1917), which was prepared quite carefully, as it were, as a summary after 30 years of lecturing.

(The last two sources justly represent an elementary summary for the scientific 'layman' on which to base a rudimentary view of Freud's theories. The second book is widely available, and the first article is available in strachey's STANDARD EDITION....)

\section{A brief view of content follows:}

"Five Lectures on Psycho-Analysis." The first lecture was historical, explaining Breuer's role and the Anna 0. case which demonstrated conversion of undischarged affects into somatic symptoms, and the existence of consciousness and subconsciousness; in the second lecture he discussed how psa became a means for recovering lost memories, and the phenomena of resistance and repression, how symptoms replace repressed impulses, but are themselves compromises between the repressed impulse and the repressing agency; in the third lecture he discussed psa technique, pointing to the parallel between neurotic symptoms and free association responses, both of which contain material from the repressed material and the repressing agency.

(It seems'obvious' that Freud was seeking the input-output chains, both internal and external, and doing this in as fresh and contemporaneous a fashion as it was being done in the physical sciences, in acoustics, astronomy, and later electromechanical science. This is not a plea to recognize the claims of the physical sciences, but to recognize the contributions of Freud, and the need to find pathways between creative science with minimal intervention.)

The analyst uses free association as a crude ore from which to detect the repressed material (very little different from dynamic physical analysis as used today, or as proposed here to expose the entire biological system operation), plus two other major interacting signal sources - dreams and slips of the tongue - named "parapraxes", pointing out that psa is to the psychiatrist like microscopic histology to the anatomist (or frequency and transient time and space response to the physicist. It is on this basis that the physical scientist can find the appeal of Freud.)

In the fourth lecture he discusses sexuality, with an account of infantile sexuality and the Oedipus complex, and conments on whether there are not essential causes of neurotic symptoms other than sexual by saying that he didn't know either, and had nothing against it, but he hadn't arranged the system and could not find any evidence for it.

(It is this point which is significant physically, that Freud, as a clinician, found none other than sexual causes for the internal neurotic pattern. If much of the internal organization represents the particular quality of the individual's neurotic pattern, then one is stuck with sexual causality. Yet we are proposing to examine a sequence of oscillator complexes, and a 'dozen' central themes of behavioral organization. Are these thoughts contradictory? We know 
too little to be certain at this time, and must simply be guided by the clinical certainty of Freud, and others who come later, to find a reconciliation for these elements.)

Freud then explained the occurrence of fixation at various points in development as a psychopathology similar to any other pathology.

In the fifth lecture he discusses phantasy as a compensation for lack of satisfaction with reality, with its outcome of better adaption to reality or creating neurotic symptoms; he discusses transference phenomena and its implication as the most convincing proof of the theory of psa.

(We have regarded this as a case for synchronization, a nonlinear property, involving a system other than linear coupled networks. It is gradually becoming clear that Freud's kcy ideas represent elements that must and can be translated into essentially non-linear network system concepts.)

He discusses sublimation as a limited adaption of the system's dischargeable energy, and he also calms fears that forbidden impulses admitted to consciousness might result in uncontrolled systems, explaining that they lose strength in consciousness.

(The common reaction to Freud was that he was modeling the libidinous man, capable of any excess if freed of moral restraint, after Dorian Gray, an elegant fiction at the end of the Victorian age.)

"The Claims of Psycho-Analysis to Scientific Interest." Psa was applicable to normal psychology as well as a tool for dealing with the psychopathology of neuroses. It had helped claim much of human behavior for psychology that had previously been thought to be part of physiology. It had restricted the physiological mode of thinking as contrasted with Freud's desperate efforts 20 years earlier to describe mental processes in physiological more strictly, in physical - language. For example, as contrasted with an older medical view that dreams were of somatic origin without meaning, now the interpretation of dreams was a key to behavior and a beginning of a "destiny of opposing official science".

(Here again is an important keynote. In tilting with physical science, Freud was mostly tilting with an academically strained, second-hand physical science, likely 50 years older, as it was available to the established members of the medical and psychological field, and almost equally remotely to the biological field. It was not direct exchange with the 'terrible children' in physical science who at the same time were also tilting with the established order.)

For general psychology, he emphasized the primacy of affective processes in mental life, and the extent with which they interfere with apparently inte1lectual processes; he also discussed the main tenets of psa. He then 
considered the relation of psa and a number of other branches of knowledge.

In philology - resemblance between the language of the unconscious and the characteristics of ancient languages, in which both appear "archaic".

In philosophy - psa poses a new concept of the unconscious, and of the body and mind.

In biology - the importance of sexual 'instinct' in psa is of prime concern to biology, but it extends to a wide range of erotic activities besides intercourse. His choice of the term 'drive' is transitional between a psychological and biological idea.

In genetics - psa points toward early life impressions as being of vital importance, and thus traces events back to dynamic, near genetic causes. These early memories remain side by side with later memories, and there can be regression back to them.

In a history of civilization - psa casts light on myths, also on the great institutions of religion, morality, law, philosophy. "There is an inner connection between the mental achievements of the individual and those of the community, since they both arise from the same dynamic sources."

(Here is the same rationale, written 50 years earlier, we propose today on physical grounds. It is clear that these earlier investigators, or even current psa investigators, wouldn't know what to do with this concept in describing the general physics of systems, but it wouldn't be fair to say that they don't have a general physical intuition about the nature of the problem. It certainly wouldn't be fair to say that Van der Pol, writing only a few years later, didn't have a very good idea of the physical connectivity. These previously general or philosophic Ideas are rushing today toward a quantifiable statement and synthesis. This study is thus only one descriptive beginning of such a synthesis.)

"The main activity of the mind is to procure the release of tension. Some...by direct gratification, but... a considerable proportion...for any available indirect forms. According to Freud,...this free amount of energy...created our various social institutions."

(This is fascinating. This idea of Freud's parallels the thesis, arrived at independently in an unpublished study, PHILOSOPHY FOR MIDTWENTIETH CENTURY that the excess of energy type activities of athletics, the arts, and scientific 'explanation', not directly connected to preservation of life activities, and thus 'leisure time' activities, was 'proof' of the internal computer nature of man. Here Freud has proposed that this essential excess of energy of the mind, which may become released in various institutional forms, represents the sexuality drive. Marry the two ideas, and there arises the concept of the internal sexually driven computer. Thus Freud has put the stamp of sexuality on the human computer. We have been searching for such a marital relation, however we are not 
yet prepared to accept it as monogamous. A sexual component

for the computer must be considered 'proved' by Freud. What remains to be proved is the completeness of the idea.)

Myths, religion, morality (and science) are attempts to procure compensation for the satisfactions lacking in reality.

(Recall in Freud's terms that this is not a necessary search for neurotic compensation but alternately a search for a better adaption to reality.)

In art - art is a form of the attempt to gratify unsatisfied wishes.

(We proposed, not at variance, that the central problem of art is attention attraction and attention holding. The reason for this was for psychologists to say. Here Freud offers a reason.)

In sociology - much social feeling derives from sublimation of particular unsatisfied erotic wishes. Undue repression leads to unsocial attitudes.

(This 1ikely was one of Freud's greatest ideas.) children.

In pedagogy - the teacher must understand his own childhood to teach

(This still remains an unfulfilled objective.)

"Introductory Lectures on Psychoanalysis (1917). The first half deals with the psychopathology of everyday life - of the meaning of the slips, and of dreams, starting from the vagueness of the material, on through the techniques of interpretation, the real and apparent content, the censorship, dreams in childhood, distortions use, etc. There is account of repressed wishes, of incestuous and death wishes. Then he discusses some of the remaining difficulties in psa - uncertainty in interpretation, farfetched quality of many interpretations, differences of opinion of others on the meaning of dreams, and differences of dreams for different analysis. The second half, of 13 lectures, ranges over all aspects of psychoneuroses, from the need to recognize the unconscious in order to understand neuroses; knowing may involve more than one part of the mind; the need for convergence of factors to produce neurotic symptoms, including hereditary predisposition, a factor of inhibited development as an organic development or as a 'fixation' of experience (these points of fixation remain foci all through life to which a regression of libidinous energy takes place, but it must be a real obstruction of sexual privation that dams the libido and sets the regression in motion). While every neurotic symptom is disguised substitution for sexual gratification, it is not an adult desire but an impulse belonging to childhood. It is a compromise between the libidinal wish and the repressing force exercised by the ego. Every neurotic symptom is the negative of a sexual perversion; which one arises depends on the degree of repression. There are conflicts between the sexual and ego group of instincts. In maintaining neurotic symptoms, the organism may make immediate or longer range gains. Freud contrasted dreams and neuroses, which have similar inner 
mechanisms. - of distorting processes, infantile wishes presented as fulfilled. In neurosis, the ego has more to repress; in sleep there is very little way to translate the wish to action. He also spends time on morbid anxiety, distinguishing between real anxiety and morbid. This solution was not complete at that time, for he still regarded anxiety as transformed libidinous energy. Finally he discussed how psa produces therapeutic results.

"A Difficulty in the Path of Psychoanalysis" (1917). A short essay in Imago was an apologia for the libido theory, related particularly to his then recent postulate of narcissism. A reason for studying sexual processes was that this was the substance of the internal behavior. Three general blows which human narcissism suffered from science were the cosmological one in which the earth was no longer the center of the universe; the biological one in which man descended from other animals, and the psychological one that man was not master in his own house, but responded to the unconscious, and now shown unexpectedly to sexual instincts.

The sections on techniques, clinical contributions, and case histories are not of present concern.

(However, the reader may already note the physical problem facing us. The case has been made for the very potent hungers in the biological system - minima11y for oxygen, for water, for food, for sleep. Thus it cannot solely be potency of a sexual hunger that can create its primacy for behavioral organization. Yet this is Freud's thesis, based on clinical investigation. Why? At the present the only thought is that the oscillator elements of the sexual system have a primacy in their ties to the erogenous zones, not necessarily potency, which makes this signal the most easily aroused signal in the body.

The best description at present is that the human is like a leaf set on the path. Small winds of signal represent a continued arousal through the erogenous zones - mouth and others - for food. This food seeking represents a drive toward self-actuation, i.e. movement of the system. There are many other self-actuation drives, many times much more powerful. However, it would appear that, like unto the leaf, the food signal blows its small, persistent, unsatiated way and continues to urge the leaf down the path to food seeking. It comes as no surprise that Pavlov's "conditioned reflex" indicates that the signals can arise and accumulate at the highest system impulse rate of near 1 impulse per 0.1 second (i.e. $10 \mathrm{cps}$ ).

Fine for a first model. Now, why sex? We can only suggest, as before, the 'accidental' or perhaps more deep-seated, this is for embryologists to say, association of the same erogenous zone, the month, for food and sex. The winds of pleasure-pain signal play over the body, almost as part of the general body awareness. Much of this signal plays over the erogenous zones as erotic and arousal signal to the sexual system. The body system is: urged down the path to general erotic pleasure seeking. The signal cumulates to levels 
requiring 'sexual' gratification. Are the other elements - need for admiration, for aggression, etc.-independent or tied to this same system? We do not know, at this time. However, the processes are likely similar and consist of an entrainment by weak forces. To coin a physical phrase, the system is in continued search for orbital synchrony; perhaps so may Freud, Pavlov and physics be joined ).

It is appropriate thus to examine the following chapter in Jones on Freud's theory of the libido. By the libido, Freud meant the totality of mental signaling impulses that represent sexual system information in the mind (he uses the terms the variable force - of processes and transforms that occur in the mind as sexual excitation). He regards it as "analagous to the force of hunger, or the will to power, and other such trends among the ego-tendencies." Freud appears to distinguish between "instincts' of self-preservation and of reproduction as a nominal description of hunger and love; and more technically between "self-preservative or ego instincts on the one hand and sexual instincts on the other." The sexual energy is distinct from mental processes in general, and in an ultimate description "it is scarcely possible to avoid picturing these processes as being in the last resort of a chemical nature" (expressed as early as 1894).

(This passage permits one more piece of speculative generalization. It would seem that the concept of instincts, and of instincts of self-preservation, holds back physical thinking. If, as Freud was willing to regard, self-preservation may be seen as a hunger, it is possible to view all 'self-preservation' systems as systems that 'self-preserve their character,' i.e. as dynamic oscillator systems, rather than static systems. One begins to view a modeling of homeostasis as regulation, and self-preservation as dynamic regulation by means of control systems and oscillator systems. In that case, the sex system is one of the oscillator systems, not different, say, from food 'hunger'. The food impulses build up to a point of requiring discharge within hours. The cycle is not necessarily fixed in time, but may vary with the social milieu. It was shown in a previous section that there certainly must be a three day cycle in the food storage complex. In an earlier section this study is certainly caught up in the dynamics of the breathing complex, and also in the dynamics of the heart pump complex. Thus why should one make a sharp distinction between the sex and other hungers? Yet it is clear that Freud viewed the other hungers as making up the ego complex, and likely distinguished from the sexual libido. Whether this is needed or not is still not clear. The purpose of this parenthetical section was to bring Freud's concept of an ego into the picture as the totality of basic internal oscillators and their states of regulation.)

Within the concept of sexuality Freud included much beyond union, and an end aim of reproduction. He described a complex development before a 
final state of genital primacy. It begins in the infant who is compelled to suckfrom both hunger, and for erotic gratification (citing earlier S. Lindner, 1879). The line continues unbroken through thumbsucking, on to amorous kissing, etc.

(Here again is confirmation, but no new light, on the thought that it is at least the common sensitivity of the mouth to food and erotic stimulation that can create the central sexual formation of behavior. Recall in (1), that much more current neurological efforts demonstrate that the early cortex state represents predominantly a control of a mouth sucking system. This may be translated to mean that part of thegenetic coding, i.e. what would ordinarily be considered to be genetic, instinctual patterns, imprints an initial state for the cortical 'slate' of control of a sucking seeking system. It is the play of inputs and the changing state of 'mind', in an epigenetic frame of psychological reference that interact to form the specific cortical development.)

By the third edition of "Three Essays on the Theory of Sexuality" (first written in 1905, 3rd edition in 1915) Freud's ideas were developed. Strachey refers to this document as Freud's "most momentous and original contribution to human knowledge." From inborn bisexuality to fixation on the mother and on things, on ultimately to sexual relief is the order of things.

(The deviations, perversions, neuroses are of no present concern. Suffice it to say, that the important added element that Freud offers is that it is the early erogenous synchrony that represents the formation of the behavioral complex. This is a most pregnant thought. One might say that the 'personality' forms around utterly trivial and formalistic elements of response in very early childhood, such as might be represented by a drop of sugar, a too warm milk temperature, a wet spot, a too rapid drop, a loud noise, etc. These trivial, accidental inputs in the early childhood likely begin important shapings of the impressionable cortex, the unconscious, etc. These things are not known as facts, but they begin to provide a common translation of psychoanalytic, ineurological, physiological, cybernetic, mathematical, chemical, and physical ideas and possibilities. The erogenous - erotic and food seeking - responses, seeking pleasures, avoiding pains, gradually begin to develop the complex of response. Therefore, the poles of behavior must exist in the internal and primitive response language of childhood. This may not be a new thought, but it represents a first key of where to start an experin mental investigation.)

In more detail, infantile sexuality develops its fixations main $1 y$ in the age $0-4$ years, the system develops latently from 4 to 11 years, and then follows the final state of genital primacy after puberty. The earliest manifestations are with non-sexual body functions - feeding, defecating, and satisfying stimuli of other erogenous zones. Besides autoerotic activity, there may be early attraction for particular persons. These early activities are divided into two phases, an oral and an anal-sadistic. (The anal still does 
not come into focus, except possibly as a mechanism to insure the inputoutput flow of food and its by-products. This leaves the system with two independent control functions - the input of food, and the excretion of waste. This could lead to trouble, and possibly does. It may require the sex link coordination to insure both functions coming off regularly.)

As a final note from this book, erotic acts institute an urge toward more intense excitation and thus imply a state of tension. However, tension, to Freud, means unpleasure since it impels to discharge and relief. Yet the acts are pleasurable, and therefore no one wants to end pleasure. This apparent contradiction took Freud 20 years to resolve.

Later papers on the sexual basis for adult behavior is at present too complex to comment on. Other work on narcissism (1914), etc. bears out significant sexual interrelations between parent and child.

A subsequent chapter 1ists Freud's theoretical contributions during this period. They include a 1910 paper on the basic and archaic significance of language and dream language; a 1911 paper on the two elements of mental functioning - the pleasure-principle dominant in childhood, and 1ater a transition to the reality-principle; a 1912 paper on the concept of the unconscious, differing from a preconscious state and the consciousness, and the existence of a barrier of repression which determines the passage of mental process to the conscious; 3 technical papers in 1915, and 2 in 1917 which sum up Freud's ideas at the time, and add some new ideas. These 5 papers are all that remain of Freud's 1915 effort of 12 essays of a "Metapsychology" synthesis. The first paper on drives represents them as singular stimuli from the body interior. Since the nervous system function is to control stimuli and restore the previous rest state, but drives intervene with a continuous stimulus, the only course the system has is action to diminish excitation.

(In (1) a considerable share of this function was ascribed to the hypothalamus switchboard in putting out fires. Thus we were only mimicking Freud.)

As to the number of drives - such as play, social, and others, he prefers to group them into ego or self-preservative drives and sexual, as a tentative hypothesis to be discarded when it suits.

(This again bears out the previous discussion. We duplicate Freud at quite a few points. Whether this arises as an original contribution, or as a subtly taught mimicking is not clear. However, the important thing is that these principles are being here rediscovered on a physical basis, and thus begin to loom into acceptability as working physical hypotheses.)

How one of these drives - say the sex drive because it had been most extensively studied - is expressed is in the following four ways: 
by reversa1 into an opposite

by turning round upon the subject (a transormation or a conversion)

by repression

by sublimation

(Bravo, Sigmund Freud! The statement made in the last report was only a tentative view that Freud was developing an information theory abstract model of the human. It becomes increasingly obvious as these essential symbolic-logical operations are found more and more as Freud's work is reviewed. In the main, Freud's contribution would currently appear to be toward a dynamic model of the internal governing information system analagous to Cannon's homeo stasis in the chemical system. The only weakness, it would appear to a physicist, was not to have cast it in a suitable abstract logical-physical system, which is the popular view of what one cannot do with Freud. Yet that this is what we are proposing to attempt, and basically in a classical physical way.)

The mental life is dominated by dual poles in which ego and outer world are linked actively and passively, and through the polar stinuli of pleasure and unpleasure.

Other essays were on repression, and on the unconscious. The latter represents a most fundamental contribution, including the concept that repressed ideas retain their energy and continue to circulate. (It is indicated that metapsychology refers to the dynamics of the total mental process.) The characteristics of the unconscious (which makes it come out to be very nearly the internal memory, however not a passive memory, but an active memory with many relations to higher centers) are no sense of contradiction, condensation of ideas, no time scale, no relation to outer reality. The preconscious and the intercommunication is discussed, as well as censorship into the consciousness.

The next section on non-medical applications of psa contain little of immediate interest except perhaps to quote amusingly, in passing, Freud's comments on trying to master material for his work on religion, TOTEM AND TABOO.

"The Totem work is a beastly business. I am reading thick books without being really interested in them since I already know the results; my instinct tells me that. But they have to slither their way through all the material on the subject. In that process one's insight gets clouded, there are many things that don't fit and yet mustn't be forced. I haven't time every evening, and so on. With all that $I$ feel as if I had intended only to start a little liaison and then discovered that at my time of life I have to marry a new wife." 
(We do not have Freud's confidence, but can understand and sympathize, having undertaken this parallel task of attempting to boil biology down to some operable biophysical principles.)

It is a1so pertinent to comment that TOTEM AND TABOO is likely a good beginning transition toward examining the primitives of social dynamics, but that is too far afield.

Finally one may note the polemic essay Freud wrote in 1914, "The History of the Psycho-Analytic Movement", in which Freud gives the background of psa and of the difference between his view and Adler and Jung.

Proceeding to Vol. 3 of Jones (59) covering 1919-1939, there is only a 1imited amount of material of prsent interest. One may note the 1922 translation of the "Introductory Lectures on Psycho-Analysis" as a useful source; the Freudian popular vogue after World War I; Freud's minor involvement in the evaluation of military medical treatment of war neurotics in Vienna in 1920; in 1920 he wrote "Beyond the Pleasure Principle", which put forth a highly speculative introduction to a death instinct (Jones proposes this type of change of emphasis as a wish on Freud's part to turn to broader philosophic questions other than the immediate scientific, and to an increasing closeness to death from fear during the war for his own sons, and from old age); a 1920 book, GROUP PSYCHOLOGY AND ANALYS IS OF THE EGO, in which Freud proposed ideas that he thought would serve as psa foundation for group psychology; in 1923 he wrote THE EGO AND THE ID, which represents a considerable change in his view of the unconscious ("it was the beginning of a new psychology of the ego, a fundamental advance in the theory of psa"); in 1925 he drafted an AUTOBIOGRAPHY which is an important source for an account of his career and development of ideas. (Freud suggested that anti-Semetic prejudice may have been contributory to the amount and unpleasant form of his opposition.) In 1939 he wrote a comprehensive essay, "An Outline of Psa", containing an account of psa and his latest ideas. From the second portion of the book it is useful to draw upon comments on some of the technical materia1. (This gets to be more difficult. The later discussion has become encased in a more complex language, the ideas have become more detailed, and as another author remarked, Freud was the only important theoretical contributor. Thus the detailed 'machine language' of the significance of genitalia and parental relations will be skipped. What is sought are clues as to the central structural organization. For the same reason comments on the MINUTES OF THE VIENNA PSYCHOANALYTIC SOCIETY, Vo1. 1, wil1 be skipped.)

By his 1915 metapsychological essays Freud viewed that he had completed his 1 ife work, and that anything subsequent would be subordinate and complementary. This would have represented a well-rounded psa in its classical form. Yet from 1919-1921 began completely new trends. "Beyond the Pleasure Principle" was the first. This conceptualization of a death instinct has not been taken to by his followers .

Freud also saw a duality of conflict in the mind. (As an organizing idea for bistable states, which can create dynamic oscillators, this is far from a bad thought). For the first 20 years, Freud was content to state erotic im pulses as being the source of mental conflict between the sex drive system and 
the ego "self-preserving drive system".

(Appropriate at this point, in discussing a 1924 paper on masochism, p. 259, Jones states that Freud had regarded the pleasure-unpleasure principle as a manifestation of Fechner's physical concept of a tendency to stability, in which tension represents unpleasure and release of tension pleasure, but had now concluded that sexual excitement alone showed only a partial correlation with this idea. "Some other unknown factor, perhaps rhythm, must also play an essential part." We do not know for certain the meaning of Jones' rhythm reference, but there certainly is an appropriateness in the context of physical ideas being attempted, in this study, to find a cyclic flow of "impulses of tension", besides an elementary erotic impulsing; particularly since a sex drive cannot be a constant overpowering impulse source, but only a gentle, even if more frequent, source than other drives. However, whether Freud can lend conviction to a death 'drlve' remains to be seen. That the death problem can grow with increasing age is clear.)

This was radically disturbed in 1914, when Freud had to postulate the concept of narcissism, and in this self-love he felt that the self-preserving drives must be included. Thus the only visible conflict was between narcissistic and erotic impulses, now both sexual drives.

(Physically still this fits quite well, even better. This is reinterpreted as follows: Freud has not included the 'oscillators' the impulse producing drives that make up the ego, within the scope of a more inclusive 'narcissistic' system that makes up the total internal awareness of self. All that such multi-worded language means is that signals are received from a variety of oscillators and integrated at some summing point, the 'narcissism' representing some particular central summation which is as yet undefined.)

This was not satisfactory to Freud, because he felt the need for another drive besides the sexual one in the ego, which he temporarily considered "selfinterest" (i.e. as if he were seeking some teleology). In 1915 he concluded that hate, or aggressive drive must exist as primary component in the ego, as well as the sex drive.

(Aha, in 1964 in the previous section, we had already 'anticipated' Freud's 1915 discoveries. The reader may be amused by this byplay. However, we are not playing games. It is a most serious purpose in this report, to 'guess' the behavioral path by physical ideas, and it is quite convenient to have a master frontiersman to follow to see how psycho-physical and psycho-medical teachings two generations apart agree. Whether the distinction of aggression and anger are separable or not remains to be seen. However, one may note that cyclic nature in sex drives, in aggressive drives, in the totality of 'ego'drives that may be considered the 'narcissistic' totality of the system, is still well defensible. Why Adler's formulation earlier was not useful is not clear.) 
Thus Freud began a non-1ibidinal part of the ego, leading to "Beyond the Pleasure Principle". In it he reiterates the importance of the pleasure principle and its agreement with Fechner.

(The abolition of excitation is identified as being very similar to Cannon's homeostasis. However, it is obviously closer to LeChatelier's principle. "The whole train of thought" Jones says, "is a remarkable anticipation of the modern science of cybernetics." True, but it is the totality of Freud's theories that are the anticipation of a communications theory. It is appropriate to find its sources in Fechner.)

However, the pleasure obtained by increase in sexual tension would seem to be a contradiction, as well as was the experience of war dreams. Some other factor, "perhaps some relation to time or to rhythm" might be acting. This Freud identified as a repetition-compulsion. Jones in passing states that 4 years later Freud clarified the pleasure and constancy concepts by stating that the constancy principle had to do with quantitative variations, whereas the pleasure principle was affected by qualitative ones also. This, it would appear, nakes the pleasure principle not an instance of the constancy principle.

(There seems to be evidence that Freud was gradually permitting a cyclicality of state to creep into his thinking to 'bind' the experiences together. The following comment suggest the validity of this track.)

Primary processes, belonging to the drives (the oscillators?) had to be bound (integrated, and in parallel fashion?) before the pleasure principle would operate. "The tendency to repetition was also pretty evidently of an instinctual nature" (1.e. drive nature) and "it also was more fundamental than the pleasure principle... and contrasted with it in its 'demoniac' character." "The tendency toward stability...was a fundamental attribute of mind." "From the three ideas...two further ones began to emerge... and they constituted his final theory of the mind."

"It was the tendency to repetition that most occupied Freud's mind at this point", the other two ideas he had had for years. "He rightly perceived that this tendency was a typical feature of instinctual life."

(That is of the drives. Paraphrased, this sentence states that the typical feature of the drives, that bound together represent the self, is the tendency to repetition. Thus the physical idea of the binding together of oscillators was anticipated by Freud 41 years ago. However, in what physical context Freud viewed this idea is not clear. At this point arises one of the trickiest pieces of reasoning.) 
The problem was how to view the tendency to repetition with that of restoring a previous state of affairs. The essential resolution that Freud proposed was that the death drive is a regressive drive to the earliest state. "So the ultimate aim of life is death". As Freud remarked, "while consideration of the repetition-compulsion was the first motive for postulating a death 'drive' it is the stability principle that affords the strongest argument for it". Thus Freud succeeded in establishing two opposing polar forces in his view of the mind, the life drives (eros) and the death drives.

(While many will consider such discussion nonsense in isolated context, one begins to detect a glimmer of the same problem that was begun in argument in the first report. An oscillator by itself does not constitute a regulated or controlled system, although it may common $1 y$ be viewed as a crude time regulator. Identifying the drives as oscillators, and the repetitive 'patterns' of their impulsive signalling input, postulates an entire system of the life 'self-preservative' regulators. However, there is the possibility of another longer range regulation. If the entire genetic structure is designed to unfold a wide range of 'maturation' type of functions, i.e. by a concept that might best be described as an entropic degradative unfolding of a single shot aperiodic network, then it is possible that the ultimate relaxational state may also be considered a regulated state, the "death" state. All of these ideas could be true, and really not understood by Freud, or even the converse. The point, is that here Freud was certainly making a very provocative, far-reaching jump, without any possible physical mechanism for comprehending the idea. Thus it will be most important to see the evidence through his eyes.)

How could this "death" impulse drive be seen? Freud saw this in the cruel, aggressive, sadistic and masochistic behavior. While at first having conceived of a death drive and a destructive drive as being independent primary elements of the ego, now he suggested that there was a primary masochistic tendency which would be an indication of the death drive. The destructive drive would derive from this. The sexual drives, responsible for the clamor of life, dir ect the opposing self-destructive tendencies out to other people (i.e. the aggressive drive follows from the death drive). Freud felt that this rounded out his dynamic concept of the mind. The summation of life drives, now termed Eros, botnd the system. The death drives led to the system's disintegration.

While Freud considered these ideas purely personal speculation, within a few years, 1923, in THE EGO AND THE ID, he came to accept them fully.

(Our comments on an 'entropy' point of view were apparently appropriate for on p. 276 it appears that Freudian followers attempted such connections, and were then greeted by devastating criticism. Yet like Shannon's use of such concepts, there is often a suggestive analogue which sometimes is useful and sometimes nonsense; whether it is nonsense here is far from clear.)

Few analysts still employ the term "death drive"; there is little objective support to be found for Freud's culminating theory of a death instinct. 
(Jones does not win on points. Freud may have had a more sophisticated view in this regard than even his followers would admit. A single drive system of sex appeared too pat and it is now clear that other primary drive systems are desirable, and reasonable. It is clear that the character of life is degradative even with care, exercise, comfort, etc. The very nature of a Gompertz mortality relation is evidence of 'catastrophic' phenomena. These thoughts suggest a possibly adjustable regulated end state, which may be actively sought, rather than a passive relaxational state. With some experiences the system subsides in its 'clamor' of sexual and life drives, and the system is recognized as having 'aged'. One may note the reference in (1) to the comment that the gonads are the Achilles heel of the system. For example, in a recently completed study of mathematical modeling of radiation mortality (16) it was quite clear that moderate levels of radiation exposure resulted in an effective life shortening experience in mortality. This must mean a physical accumulation of impulses that are increasingly destructive. There always seem to be psychological inputs or effects than can produce the same physiological results as physical inputs. Thus it is not impossible to accept the thesis, at least as hypothesis, that there is a component of mortality that is psychologically produced. How much, is not clear at this point.)

This brings the discussion up to THE EGO AND THE ID (1923), or a more popular exposition in NEW INTRODUCTORY LECTURES ON PSYCHO-ANALYSIS (1932). Nut as revolutionary as the death drive, Jones considers the thoughts developed here in the main line of Freud's ideas. The Id is Freud's Germanic "Es", meaning the "it", a non-personal part of the mind distinct from the narcissistic and egoistic self. The Id is a primordial reservoir of energy derived from the primary life and death drives. It is an unorganized drive, thus differing from the organized ego drives. It has the negative attributes of the "primary system" concept that Freud used to replace the unconscious with no negation, or contradictions, or time sense, etc. This non-personal Id is broader than the previous unconscious, which had been synonomous with what was repressed. He was driven to believe that the ego contains not only the elements of self (and its awareness), but continues deep in the unconscious.

Thus, more accurately, while the kernel of the ego still was the sum of perceptions received from outside, now it was that part of the Id which had become modified by these perceptions. The important part of the perceptions, the most affective, involved the human environment, typically the parents, and Freud lengthily described the nature of these imprints.

(For a crude measure of such ego imprints, in an objective attempt to understand these difficult formulations in some experimental frame other than Freud's, Gesel1's THE CHIID FROM FIVE TO TEN was examined and in particular the number of 'imprints' that Gesel1 describes as marking the behavior profile of a child of five. The intent is to note whether the concept of ego imprints remains consistent with oscillators, or cycle drive mechanisms in the system.

Stripped into a minimal sketch, Gesel1 notes: 
The child of 5's external world is -

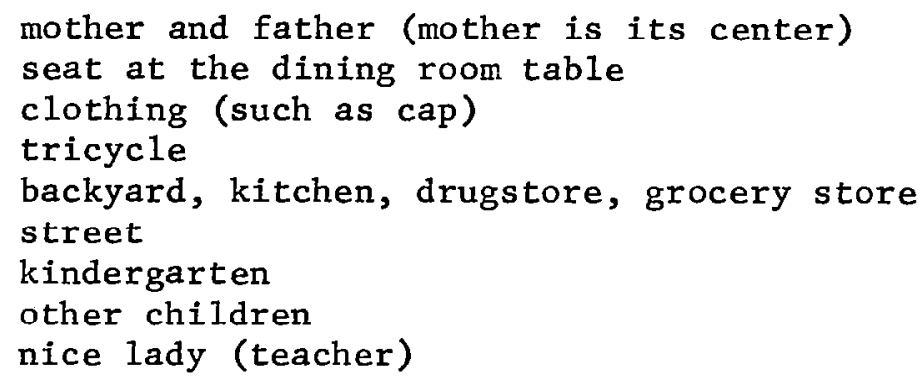

These are elements of food, thermal protection, comfort and security, locomotion, and of a broader undifferentiated world.

His play role (i.e. a continued internally derived response complex that the system seeks to reproduce) is at playing house, not in a stereotyped fashion but with small variations, and reliving the experience of early childhood by recourse to the mother's memory.

Emotionally, he is adapted to his local time and space, he is moderately competent, he accepts help, responsibility, his actions are self-limiting rather than aggressive, he is docile but not socialized, he has a vein of humor, he is strongly linked to his mother, he is pragmatic, he is a great talker.

His traits (i.e. the behavioral elements, which are the present major concern as illustrative of ego imprints of the environment) include:

Specific patterns of motor activity (posture, gross motor activity, alternation in play tasks, active but with economy of motion, eye-hand performance, moderate hand performance, copying).

Eating habits (good 2 meal per day appetite plus a poor third, complete eater, likes simple food, willing to try a few new foods, self-feeding, reasonably capable if not thrown into a complex family meal situation).

Sleep habits (requires about 11 hours sleep, and a nap, with little other distraction. Elimination functions may or may not invade sleep hours. Dreams and nightmares, predominantly about animals; child quiets at parent's voice).

Elimination habits (one movement after eating, urinates infrequently and tends to put it off)。

Bathe and dress (they can but often don't).

Health and body complaints (childhood communicable diseases, and stomach aches from food and elimination characteristics).

Tension outlets (thumbsucking has terminated, some comfort objects are used, some hand to face responses are used or cranky responses are used). 
Emotional expression (stays close to home; speaks deliberately; is helpful; poised; inhibited; can't stand contradiction, not vaguely afraid any more, only of certain things such as thunder, rain, and the dark, and above all of being deprived of his mother; dreams of wild animals and strange bad people; secure in world around him; has a remarkable memory for the past).

Sex (his interest is in the baby, not in sex. Sufficlent to know that it comes from "Mommy's tummy". Doesn't connect it with growth. Interested in the play drama of having a baby, or having been a baby. Has become personally modest. While aware of sex diffferences, does not have a great deal of curiosity or interest).

Interpersonal relations (strong tie to the mother, but with some share of affection - fondness, pride - in the father; won't take punishment as well from father. Plays well with children in a sma11 2, at most 3 , group).

Play (he likes play with all kinds of materials; he plays with and about houses; he plays using his body in athletic type activities; he likes stories; he likes music).

School (can be with children in a supervised group, and enjoys a routine of activity; can pick out letters, and numbers; language during group work tends to be repeated from person to person).

Ethical sense (his poise is sustained by a balance between his needs and environmental demands. His ethical sense is related to this, and is very new and tentative. He responds somewhat passively, being so adjusted, and is generally considered to be very good. However, he doesn't distinguish right from wrong, mainly acceptable and unacceptable behavior. He is relatively truthful. He finds external reasons and things to blame).

Philosophic outlook (God is accepted in a matter of fact way, as is death. Time for him is his now and is quite personal, as is space).

This description certainly represents both a sum of a limited number of perceptions received from outside, with its most effective part the human environment, and the unconscious internal enfolding and development of drives - typically oscillator systems, but becoming disguised - in response to and in conjunction with these perceptions. It is as if the system, in its various states of unfolding, locks on or synchronizes with that portion of received perceptions that it is reasonably capable of coping with i.e. an orbital synchrony. A filtering, cutting out, repressing of an entire range of possible affects is what is most characteristic of human and other bfological system behavior. However, it is clear that the ideas being developed are meant to be of most primitive forml. 
Returning then to Freud. Whereas Freud had originally viewed the inputoutput process as a primary narcissism of the ego (internal awareness of the drives) from which libido (erotic impulses) stream outward, and in being withdrawn from it, represent a secondary narcissism (i.e. an awareness of both output, self, reflected input), now the libido of the Id is directed outward to seek gratification, and, if failing, is redirected inward but desexualized and sublimated. Inasmuch as the sexual goal has been given up, narcissism and sublimation do not serve the life drives, but belonged to the death drives. "This was a further complication in his instinct (drive) theory".

(Here we are lost in what is becoming metaphysical and vague. The fault may be ours, Freud's, or Jones'. One gets the straightforward idea that Freud still wanted the "erotic" stream of impulses to continue to pour out internally; that these arose from the totality of internal drive systems that make "it" - the system - up; that the gross 'motorized' system is urged to seek pleasurable erotic response; that it will accept less or other input; and now the thought that neither the internal awareness nor the sublimated input are part of this drive bound system, but are part of the final state regulatory system. However, both Jones' and cur concepts are far down the road from direct evidence.)

Finally, following Nietzsche, Freud postulated a super-ego, and ego ideal in the mind which censors behavior, as a moral conscience. The super-ego is in part unconscious. (How much of this is to be regarded as science and how much Hegelian metaphysics is hard to say.) The evidence, generally, is the unconscious sense of guilt which can impose great suffering. The ego can defend itself against this suffering by its weapon of repression. This is the reverse of the familiar one in which the ego represses impulses at the behest of the super-ego.

In spite of this being Freud's primary contribution to the super-ego concept, it was more complex than at flrst suspected. For example, in a 1924 paper "Neurosis and Psychosis" Freud summed up by saying that psychoneuroses are the outcome of conflict between ego and id; narcissistic neuroses, such as melancholia, between ego and super-ego; and psychoses between ego and outer world. In a second paper, he pointed out the greater influence of the outer world in neuroses, and of the $i d$ in psychoses. In the latter, impairment of reality is inherent. In the former there is an initial flight from the demands of the id, but these impulses continue, and a compromise must be reached which represents a secondary impairment of reality.

(However, it appears Freud was not satisfied with his conceptualization. At this time in another paper he states, "Whence does neurosis come--what is its ultimate...meaning? After whole decades of psa work we are still as much in the dark about the problem as ever".)

In a 1931 paper, "Libidinal Types", he attempts a classification of humans by their libidinal character, as erotic, narcissistic, and obsessional. In the first, a love life, the desire to be loved, is a main feature. In the second, the desire to love is stronger than the need to be loved, but both are subordinate to self-preservation and self-assertion. Little tension exists between ego 
and super-ego, the latter is not highly developed. Such people are leaders, and aggressive. Obsessional types are dominated by their super-ego. They fear conscience more than no love. They are conservative. Most people are a mixture of two, erotic-narcissistic being most common. An ideal harmony would be a triple type, which is essentially non-existent.

Somewhat later one could say that Freud was definite in two conclusions. One was that the super-ego has a closer connection with the Id than does the ego. It originates in the inner world, the ego in the outer.

(Does this mean that there is an internal oscillator, or a circulating core memory, a higher algorithm that represents the super-ego; that there is an entire abstract central engine system that represents a prime mover programmed to keep the system going, that represents the summation of life and death drives of the id; and that finally there are the driving impulse-producing oscillators whose totality represents the ego?)

The other was that identification with parents, particularly the father (remember the pride and fondness of the 5 year old), plays an important part in its genesis. When the Oedipus complex, the primary erotic or sexual fears of the child - whether boy or girl - are resolved, then the father image begins to emerge clearly, and the super-ego becomes the heir. The child's identification is not with the parents' ego but their super-ego.

(One wonders whether behavior is not modeled after the grandparent, which poses the interesting line that morality is formed by the parent, but behavior - which cannot be too far different - is formed by the parents' romanticized memories of their parents.)

However, the identification is not perfect. The child's distortion is his own.

Freud's contributions, in general, to biology are considered to be the concept of a drive transitional between psychology and biology (also the hint that "ontogeny repeats phylogeny" may be true of mental processes too); a possible foreshadowing of the neurone theory; the likely evolutionary nature of many of man's mental activities and beliefs; the central role of instinctual behavior; mental mechanisms of displacement, regression, etc. are found common in other animal behavior; and a classification of instincts into libidinal and aggressive instincts. (However, there is no evidence of any instinct toward high moral, ethical or spiritual aims.) One weakness of Freud's, that Jones alludes to, that he remained an obstinate adherent of Lamarckism, the inheritance of acquired characteristics, though he had said that Darwin's theory of evolution had motivated his choice of scientific career.

Remaining portions of Jones, Vo1. 3, are not of present concern. Thus a brief summary is in order. 
We had proposed to examine Freud for clues touching on the general concept of the behavioral system as a computer complex. In this study, involving the biological control systems also from a physical control point of view, it emerged that Cannon's homeostasis had already anticipated the concept of regulation in the chemical content of the system. Further examination of the system led to the suspicion that it was organized as a bound together system of oscillators, and thus the ideas were joined in a 'novel' idea that the entire behavioral system was an internal computer controller for regulatory purposes of this oscillator complex. We found that Van der Pol had contributed significantly to the individual oscillator conceptualization. We now find that with mild translation these psycho-physical ideas had really been considerably anticipated as psycho-medical ideas by Freud. Freud's claims to fame are thus quite enormous. (Whether perfect in all details or not, is irrelevant. His works must stand like Newton's and Einstein's.)

Having been able to track 'independently' a considerable amount of Freud's ideas by what is purely physical reasoning about systems we are thus encouraged to continue to pursue this path, of an independent effort that occasionally touches base with other investigators.

Thus one may note:

1. A system of oscillators (Freud's drives) each of these representing a complex spectrum in their own right, and not independent (Freud's ego). This physiologically derived idea, Freud found, essentially lay at the foundation of mental activities.

2. One or more organizing complexes of a general 'computer' nature. (Freud suggests two, an id and a super-ego.)

3. System regulation (Cannon's homeostasis, Freud's "stability", "Ninirva principle", "Fechner's principle", or more apt, LeChatelier's principle.) This is a physically complex problem which we feel competent, in the long run, to expose (th is suggested that this is perhaps better identified as homeokinesis).

For a rapid view of Freud from someone who differs with Freud, a short book, Fromm's SIGMUND FREUD"S MISSION (60) was quickly reviewed. The following points were noted: Freud felt he was developing a political-philosophic-scientific religious movement in psa. Its central dogma is in THE EGO AND THE ID. Fromm quotes from this, "Psa is the instrument destined for the progressive conquest of the Id", a religious-ethical aim, the conquest of passion by reason. Thus Freud was similar to Marx, who felt he had found a scientific basis for socialism. Under the guise of science, Freud was playing at Moses to show the human race the promised land, the conquest of the Id by the Ego. However, the religion had the fate of most such movements - schisms, a hierarchy, dogma, ritua1, idolization. "In fifty years, there has been relatively little theoretical development beyond Freud's own theoretical innovation", except for the Iife and death instinct, which did not compel Freud to undertake the drastic revision of his earlier mewhanistic views. While this deals with the content of Freud's idea, it does not deal with his discovery of a new dimension of human reality, the unconscious. However, Freud bogged this down in libido and 
repression, and "little or not at all to the wider reality of human existence and to social and political phenomena". Most psa are not less blind. Thus Fromm states that the understanding of the individual unconscious presupposes and needs critical analysis of his society. Freudian psa remains middle-class. In summary - in 1900-1920 psa helped to end the Victorian standards; after 1920 they represented no challenge. Psa became a substitute among 1iberals for a way to find a meaning to life, a surrogate for religion in the middle and upper classes. It is wrong to say that psa promoted sexual liberty. The wish satisfactions of the middle class in the 20th century promoted a market for consumption of all gratifying commodities including sex. Yet World War I and II, Stalin, the Nazis, represent steps in the defeat of reason and sanity. Freud was the last great representative of rationalism, even more tragic is the closing of his life drama in the Hitler and Stalin madness. However, in the unconscious he also struck a fatal blow against rationalism. Like Moses, after the defection of Joshua (Jung) he knew he would never get to the promised land.

(Eric Fromm is considered one of today's most brilliant social psychologists. Yet we must believe his analysis is nonsense; it may be brilliant, but nevertheless nonsense. We can question even whether there is a 'real' comprehension of Freud. Though there is no interest on our part in joining Freud's 'religious' movement, we can find no other set of dominant ideas that would be useful, at least as viewed from the eyes of middle-class 20th Century American physics!)

We could review much more original material of Freud's, but to run through 18 volumes of GESAMMELTE WERKE, 12 volumes of GESAMMEITE SCHRIFTEN, 24 volumes of the STANDARD EDITION has no point at this time. Suffice it to say that we ran through the thin 5 volume set of SIGMUND FREUD, COLLECTED PAPERS, Basic Books, 1959, taken from the earlier Hogarth Press edition. Most of the main points of interest have been covered in the commentary on Jones.

As a next general reference, for theories of the mind, i.e. of the behavioral complex, the American Handbook of Psychiatry (61) was reviewed.

In Chapter 2, on Recent American Psychiatric Developments, one could pursue a line of development from Cannon's recognition of the physiological changes in pain, fear and rage to Karen Horney, to Selye's stress syndrome and an effort to a general systematization of biological adaptions in the endocrinological secretions. (In the hypothalamic-sympathetic-adrenal medullary system and hypothalamic-hypophysial-adrenal cortical system.) Selye has had limited influence in psychiatry. Interesting papers on an experimental approach to anxiety have been written by Rioch and Grinker. On experimental technique, more generally in psa, the work of Lokubie deserves investigation. The theoretical work of Rapaport may, also be noted.

Chapter 17 on "Obsessive Behavior" by Rado appears interesting. The most notable thing is the periodic "swinging back and forth between the same set of pros and the same set of cons without being able to reach a decision". Yet the compulsive acts are trivial, and essentially a ritual repetitious sequence of motor acts. As Rado's rough sketch of the obsessive patient indicates, he does not appear so unusual. After sketching out a Freudian psychodynamic foundation, 
Rado turns a chain of psycho-dynamic inferences back to infancy, and finally decides that the neurosis arises from prohibited sexual desire plus defiant rage versus fear of conscience plus guilty fear. It is based on a predisposition acquired in childhood involving five discernible factors - very strong rage; guilty fear made stronger through the repressed rage; a very strong primary feeling of omnipotence; pleasure deficiency in sexual orgasm so that repressed rage cannot be thus discharged; sufficient intelligence to have realistic fears.

(Without going into the question of the validity of the diagnosis, it would nevertheless seem obvious that the factors under discussion are precisely many of those that have been here identified as elements of the oscillator systems, and that what is described appears to be interaction among these oscillators. The neurosis itself appears to result in an oscillating instability that itself involves commonplace elements of normal 1ife. It is just certain but uncalled for 'compulsive' elements of the oscillation that indicates something is wrong. All of this adds up to a non-linear instability, as has been here generally but yet vaguely proposed as the general mode of physiological, and now psychological operation.)

In Chapter 22 on "Maniac-Depressive Psychosis" by Arieti, the psychosis is defined as a group of mental disorders characterized by periodic attacks of melancholia or elation of rather marked proportions, accompanied by retardation or hyperactivity.

Arieti states that one has to turn to psa to get beyond a descriptive concept of the condition, with the explanation first coming from Abraham, then Freud, Rado, and Klein. The disorder seems to have declined in frequency, suggesting the possible significance of social and cultural factors. Electric shock treatment is capable of producing a temporary recovery from a depression. In cases of mild depression, intensive psychotherapy may produce a transference to the therapist as a new and reliable love-object, but it doesn't last.

(One begins to wonder whether any psychiatrists will be struck by the apparently repetitious theme of system states that are oscillatory and thus 'unstable' statically, but stable in a non-linear sense. The limit cycle character is indicated in that the oscillatory state does not depend on how the system is started; once the conditions for instability are formed the system approaches its limit cycle. Here, we believe we have struck pay dirt. The chain extending from the analysis first of the autonomous oscillators in any complex physical system to get them out of the way, to their conmonness in the biological system, to their central role in all control systems, to their central role in the organization of the biological system, to their occurrence in the psychological system, to their central role - translating Freudian concepts - in the structure of 'mind', to their 'obvious' nature - at least to a physicist - in mental disorders has come a long way since the start of this program. Yet it is the only reconciliation, the concept of various non-linear cycle states of the system, that will permit psa, chemo-therapy, and physical concepts to exist side by side in explanations 
of the same behavior. One can only respectfully request forebearance as the structure unfolds in further studies, and as the system whose elements contribute to these various oscillator states is explained. One requirement will be to drop the heavily laden metaphysical language and seek to find the simplest physical description of symptoms and mechanisms possible, dropping out all irrelevancies of description. Less details, more key ideas are needed.)

Chapter 23 on "Schizophrenia" by Arieti, is a very helpful focus. It was clear, say in a large 1957 meeting devoted to the problem, that no satisfactory integrating approach exists, and that there was not a single concept on schizophrenia that was not seriously challenged. Schizophrenic reactions are a group of psychotic reactions, often beginning after adolescence or in young adulthood, characterized by fundamental disturbances in reality relationships and concept formations. After Kraepelin, insight into the problems is attributed to Bleuler (1911) who accepted Freudian dynamic concepts in $h$ is formulation. Meyer then stressed the "longitudinal" life study of the disturbance. In 1903 Jung began to apply psa techniques fully to schizophrenia. In 1914 Freud applied the libido theory to schizophrenia. The libido is withdrawn from external objects and is divided into the ego "narcissistically". Many other investigators continued, pursuing various lines. A general description of the disorder is a lessened interest in life and an obsession with certain specific problems. The subject deals in bizarre ideas which have to him a special frame of reference. These are delusions generally of a negative character. The perceptual functions of the subject are altered, in a distorted way. There frequently are hallucinations, without external stimulus or object. These involve every sense-auditory most frequently. At times the general behavior appears normal. The mood and affective sphere can then alter. The subject withdraws into a she11. "The general behavior appears odd in many ways: mannerisms, grimaces, purposeless acts, stereotyped motions, impulsive gestures are observed."

(At this point our key thesis is fairly demonstrated. The status of the biological system, whether physical or mental, at any period of its development, whether so-called normal or abnormal or disturbed, is to be described by a loosely coupled grouping or non-linear limit cycle oscillators. From this system stems the homeostasis, the control characteristics, the reflexes, the systems behaviors, the drives, the ego, the responses - chemica1, physical and psychic, the normal, pathological, psychotic, and neurotic states of the system. This may be regarded as trite; it may have been anticipated and done by others - it will be examined in greater detail in later discussion, of Rappaport. Nevertheless the central idea, as a key physical idea for organization, is true. It is likely that the line being generally followed here was anticipated to some extent by Van der Pol. For example, the complete artificial nature of schizophrenic states points to a limit cycle oscillator that is shifted to an artificial 'non-normal' state by some sort of stability criteria. 
This provokes another passing thought. A boundless admiration must be expressed for the psychiatric scientist, particularly the psychoanalytic, for having managed to put together qualitative verbal elements of a valid description. It is sad to see this, and recognize that what was lacking was the physical-mathematicallogica1-methodological apparatus for converting this to a good quantitative base. It should be possible to accomplish this today.)

The course of schizophrenia is as an aperiodic series of attacks (as if the relative stability of the system were intermitently affected - one can believe, if necessary, that such shifts could be chemically mediated). Psychodynamic studies reveal that the road to schizophrenia begins in the remote past, perhaps shortly after birth of the subject. The troubles start in early childhood. A vivid impression to the analyst is that the patient's family, and the parents' marriage, is not a happy one. The marriage is skewed and accepted so by both partners. While not specific for schizophrenics alone, there is conflict, tension, and anxiety. The entire family unit is destructive, and maintains considerable delusional folly.

(This al1 leaves open possibilities in the system structure, in chemical factor mediation, in the early childhood relations as leading toward, or creating the limit cycle instability, or even as assisting in establishing conditions of an orbital synchrony. At this time we are not playing at psychiatry, just pointing out the actual structural elements that might be assembled to making oscillatory systems. It is quite clear that the relation between child and parents is a state in which they are both attracted into synchronous orbits. This wasn't clear in the last report. Now with this young system running a synchronized course under the mother's tutelage, the question is how the oscillator elements are integrated into a broad, synthesized whole. It is clear that physical elements - particularly chemical ones - must do most of the fixing. It is clear that certain maturation elements must unfold into the path of this synchronized frame, but none of the details are clear.)

In Chapter 24, continuing, Arieti points out that specific chemical agents seem to have engendered syndromes similar to schizophrenia, and he points out a number of times that Kraepelin had sought to place the disorder on metabolie foundation but with no corroboration. Some psychosomatic description of cortex mechanisms in schizuphremia is attempted, involving the TOP (mostly temporal, a little occipital, and parietal) and prefrontal areas of the cortex, their action on the archipalliun, with its control functions of the hypothalamus. The somatic and biochemical alterations found in schizophrenia "would al1 be consequences of the functional alterations of the hypothalamus and of the encephalic vegetative centers..." (which comes back to the theme discovered in (1) of a hypothalamic switchboard mediating the action of many internal oscillatory systems in the body). Shock and drug theapies do influence the patient. (However, we have heard Dr. Douglas Bond discuss the near hopelessness of cure.) The difficulty of obtaining transference to the analyst because of the withdrawn libido has made the problem of psa treatment 
difficult. One idea described is that the schizophrenic is one who has been deprived of the experience of a real mother, and a psychiatric nurse may offer that love. (This one 1llustration 1s, of course, very selective, but it points up the synchrony idea.) At any extreme, Szasz is quoted as saying that although schizophrenia may be considered to be the core problem of psychiatry, like the problem of the ether there may be no such problem. Arieti thinks that there remains a core of elements in a schizophrenic syndrome, and thus in closing, defines it as a specific reation to an extreme state of anxiety, originating in childhood, later reactivated by psychological factors. The specific reaction involves a predominant adoption of mental mechanisms from lower levels of integration. Since the result is a regression to lower levels, but not integration, regression takes place. (The switchboard disconnects from 'above' and sticks, the record plays lower routine themes over and over, and the total system disorients further.)

Pursuing these themes in later chapters, following official classifications from the source DIAGNOSTIC AND STATISTICAL MANUAL of the American Psychiatric Association, such as the delusions in paranoid conditions, melancholia, exotic syndrones, is not of present concern.

One interesting note is abstracted from Chapter 29 on Deviations in which Sperling is quoted as saying that in a group perversion there is a Leader who is a genuine pervert, the followers participate under conditions that are ego-alien, but the fact that they do so indicates the presence of a super-ego that permits substitution by a leader. (Emphasis again on orbit attracting synchrony.)

In Chapter 33 on Psychology of Cardiovascular Disorders, the authors point out that it is a well known fact emotional stress can cause measureable changes in physiological parameters like blood pressure, heart rate, stroke, and resistance, (as was noted in Biderman (58)) illustrating with a study by Hickam et al who demonstrated a doubling of mean metabolism during a period of mental stress, essentially anxiety, compared to a period of relaxation. (Considering the earliest sections, in which the factor of two to ten changes were cited as a typical example of the range changes that might be expected in oscillators used as regulatory mechanisms, this illustrates that psychological factors can also be involved in the stability considerations involved in oscillator complexes, as influential in range change as any other factor.) However, the feedback illustrations in this chapter, for cardiac disease and hypertension, do not make much impression on us.

Similar thoughts are involved in the chapters on gastrointestinal and respiratory disorders.

In a later chapter on The Body Image, it is stated that the body image is integrated physiologically in the parieto-temporal areas of the cortex. Cook and Druckemiller have suggested that it is represented in the cortex as the function of a widespread neural network in the postrolandic area. 
In Chapter 45 on a General Theory of Communication, Ruesch, raises the question whether the convergence of scientic thinking in communications (engineering)theory, and psychology isn't due to the central need, at the end of the scientific road, for communication (tentatively we say no. We believe that it is due to the really limited number of different concepts, topologically, that the human can use to deal with in abstract problems, and hopefully, that nature wasn't really able to drcam up very many more so that they can be put into one-to-one correspondence). In psychology it is unique that subject and investigator are interinvolved. (Again, no, since quantum mechanics, or really since the disappearance of the ether, and relativity, this point of view has been forced into physics, during the same period as Freud's development. It is more to the point really to note the similarity in development of 'modern' psychology and physics.) It is of pointed value to the analysist to stick close to simple formal empirical abstract elements rather than attempt symbolic transformations (True, but real progress takes place with the insight into unifying generalizations)。

A few minor notes were made on Vol. 2 (61). In Chapter 62, psychiatric association with metabolic, endocrine and nutritional disorders are discussed by two hypotheses, the first of a causal connection between such chemical factors disrupting mental functions, or the second of indirect effects on an integrated network of ego, homeostatic conditions, body-image, The first has always had logical and evidential deficiences. The alternate is rooted in discoveries representing approach of psychiatry and the social science as field theories. (Yet the reader should note from the first report on that it seemed reasonably obvious from a physical point of view, that chemical factors might be best considered factors that shift the operating stability of oscillators. In this report, the hypothesis has arisen that the entire psychological ecomplex is oscillator organized, so that chemical input would still remain the same. As an illustration of part of this thesis, we note a 'popular' report by the medical writer in the Cleveland Press, March 27, 1964, "A 25-year study at the University of Pennsylvania showed that happiness runs in cycles. A teen-ager's joy cycle may be as brief as two weeks; an adult's may average from 33 to 36 days. Results indicate that the pituitary and thyroid glands, liver and other organs that produced secretions essential to the feeling of well-being also varied from week to week".)

The self is recognized foremost as a body-ego. Patients given significant hormone doses feel 'different' without objective evidence of change. In lower animals, the effect of hormones on behavior is quite clear. However, the mechanisms of effects are not known. Thus a unique correlation of hormones and behavior is not tenable. The factor of timing in the picture of maturation is significant for effects.

After illustrating psychiatric associations in a variety of physiological diseases and conditions, Selye's concept of stress, and its modification, and relation to many psychiatric syndromes are mentioned. (Our strongest impression, over and over again, is that many of the investigators named or referenced, such as Selye, as Freud before, etc. have been most certainly on the right track. However, they bury the answers within a pile of words. It is a pity that they do not make use of a mathematical-physical-logical abstract training that could likely have helped boil the psychological problems down to its 
essentials during the past 40 years. Occupied as they were directly with the content of the problems in direct clinical experience they were infinitely better prepared to squeeze out the experimental evidence from their past daily experience than we are now, who will have to seek the evidence piece by piece from the literature, from primitive experiments, while at the same time trying to learn the clinical frame of reference)。

In Part 10 on Psychoanalytic Therapies, Chapter 69 on the classic Psychoanalytic Approach is appealing and instructive. Freud's libido theory of psychosexual development is outlined in the child: an oral phase of pleasurable body activity - mouth, lips, tongue, stomach - for 18 months; an anal phase - anus, rectum, bladder - of voiding and retention pleasure during 18-36 months; a phallic phase in the 3-7 year range; a latent period from 7 to 12 years; physiological changes in puberty 12-14 years ushers in the adolescent upheaval during the 14-20th years. In the dynamic point of view of psa, human behavior results from the interaction of instinctual impulses seeking gratification, and counter instinctual forces opposing gratification, in favor of security or self-esteem, etc. (Again, basically very good ideas drowned in an ocean of words.)

In Part 11 on the Physical Therapies; in Chapter 74, insulin shock therapy is considered the most accepted somatic treatment for schizophrenia, leading to considerable improvement in illness durations of less than 6 months, but leading to essentially no favorable response for patients who have had the illness more than 2 years. The relapse rate is high. (This, in our opinion, is an example of something common in biology, of the use of potent chemical affectors of dynamic oscillatory systems complexes, in particular of the system stability, without the affector really being 'right' for the end change sought.)

In Chapter 76 on Psychosurgery, it is indicated that the frontal lobe is the prime location for operations to relieve mental disorders, with 1ittle results obtained from TOP lobes.

In Chapter 77 on Drug Therapy, it is indicated that while they do not have causal effects, a considerable number of drugs have been introduced in recent years that are very effective symptomatically. Many others have been used with controversial efficiency. Tranquilizers are used in mania conditions, and not indicated in depressions, and have become very common in schizophrenia. Clinically,they relieve excitation and tension, and thus appear to have a sedative action. They reduce hallucinations and delusions. They suppress symptoms, but don't eliminate structure of a psychosis. They are active in the midbrain and in the reticular substance. Details of action are not clear. Clinically, they likely sedate the midbrain, and permit the nervous system to cope with stimulation (in an elementary sense, the description resembles disturbance of the usual time scale of interference from a higher command center, so that the supervisory oscillatory dynamics slows down).

It is now clear that a search for mechanism to support our ideas must be pursued in neuroanatomy and neurophysiology. It is most appropriate thus to find the 4 Chapters 79-82 on Neuroanatomy, Neurophysiology, Neurology, and 
Neuropathology. The subject is so detailed and important that it is pointless to attempt a view by a few casual abstractions. This will have to develop further in future effort. Yet a few points may be abstracted from Chapter 80 , Gerard's section of Neurophysiology. "Brain begets behavior" is offered as a truism. In describing neuron interactions, both sequential aperiodic reverberating loop signalling, and parallel rhythmic behavior is recognized. Feedback is recognized, as is synchronization, both by electrical and chemical fields. Desynchronization is possible by chemical signal at neuron thresholds. Neuron rhythms likely quantize temporal experience that "last about a tenth of a second." (There is little doubt that neurophysiology has a better grasp of the physical problems of the biological system as exemplified in the nervous system than anyone else. However, it is not clear whether biological scientists, including psychologists, would accept or have accepted that the outline of detailed communications and physical network response shown in the nervous system - which is only clinically 'known' by empirical sympomatic observation but not by any adequate mechanistic descriptions - is likely common to all other biological system organization.)

In discussing reverberation, Gerard uses the phrase "structure has been called frozen action", and in general makes the point that biological memory fixation, particularly at synapses, are activated many times by reverberating impulses, and may thus be the clue toward system change (while partially only analogous, in a recent study contract on hydrology (62) the theme was developed that yesteryear's rains developed today's rivers, showing how the river flow was proportioned to that river system. In another recent study of the physical foundations of hydrodynamics (10), a unified description of the underlying physical ideas in the statistical mechanics of fluid systems was brought together. In both systems, as in the nervous system, it is necessary to recognize the number and kind of underlying forces that create the detailed elemental substructural changes, if only in a phenomenological sense to get an idea of how the larger system undergoes change, and what its apparent dynamics are). Gerard points out that it is likely that all sensation, including psychological, depends on at least a few reverberations, illustrating with common recognition in a twentieth of a second, and novel recognition in more nearly a tenth of a second. "The whole problem of consciousness can be examined from this same viewpoint". (Once again, one sees the near $10 \mathrm{cps}$ fundamental rhythm, as being the level of the highest frequency organizing element in the dynamic complex of the multicelled biological system.)

The final section that is pointedly significant is Chapter 87 by Rapoport on Mathematics and Cybernetics. The author proposes to examine the pertinence of a number of modern physical-mathematical subjects (information theory,etc.) for medicine and psychiatry, in particular the neurological basis of behavior. obviously physics, chemistry and biology have supplied direct tools and methods but there may be more contribution of the basic 'disinterested'sciences to the applied 'practical' sciences, most expectedly of physical science to engineering, and biological science to medicine. What is the content of 'behavioral science" that would similarly contribute to psychiatry? Cybernetics, as an engineering science oriented toward communications engineering rather than power engineering tends to make the former behaviorally oriented. (This is the Wiener thesis, which was reviewed in the first report (1), that regulation 
and feedback control theory will be useful in exploring biological system action.)

Rapoport first examines the possible contribution of servomechanism theory. Purposeful goal seeking seems to be characteristic of 1ife, as compared to a mechanistic unfolding of both in ordinary physical systems. To vindicate a mechanistic view of nature requires physical theory of machines with 'purposeful behavior'. Machines capable of apparent goal-directed behavior are called servomechanisms. This branch of engineering "has been christened 'cybernetics'". Simple types of 'cybernetic' machines (regulators) were known early in the industrial era. The central principle of cybernetics is the utilization of the discrepancy between the actual and the prescribed to control mechanisms. In the flow of influences, there appears closed feedback loops. "A servo responds not only to the outside world but also to its response to the outside world, and therefore to its response to its response, etc. No doubt it is this circularity which gives to the behavior of servomechanisms this sometimes amazingly 'animate' results". To the uninitiated, servos would appear purposeful or intelligent. Similarly,living things behave in some ways like servos. This analogy gives rise to many useful ideas in behavioral terms - not as conclusions but as central concepts. For example, the servo transfer function concept leads to an inverse problem - given a transfer function observed in performance, to infer the internal mechanism. This is the psychologist's problem. However, any internal arrangement may not be unique. It is hopeless at this stage to attempt to infer the brain structure from watching arbitrary stimulus-response pairs. However, some investigators, Wiener, Stark, undertook eye-motion tracking problems by servo theory。 (As the reports in this study have tried to discuss in many ways, Rapaport's remarks are wrong.)

1. It is naive to consider servos as goal-directed in any sense resembling a complex human. It is like saying that a burning match after all represents the same processes as a human's metabolic regulation.

2. The author can christen servomechanism theory as cybernetics. Workers in the fleld of automatic control will not. Cybernetics as a theory of governing mechanisms had as its purpose the application of a communfcations theory, including feedback, to biological systems. The communications theory of feedback was developed in the 20 's for electronic circuits. The electromechanical and mechanical theory of servomechanisms was developed during the late $30^{\prime} \mathrm{s}$ and early $40^{\prime} \mathrm{s}$. The basic network theory of both mechanical and electrical form, with the central idea of coupled networks of which 'negative feedback' is one case of coupling, goes back 100 years, and is quite well contained in the theory of normal modes of network systems.

3. Though Wiener indicates clearly knowledge of the distinction between regulation and control, it is doubtful that this emerges here. The regulators from Maxwell on may all better have been viewed as force balance devices - like a mass-spring combination - which can find an equilibrium position, rather than as cybernetic machines that 
demonstrate purposeful behavior. As the point was made in the first report, it appears doubtful that many non-physical scientists are aware of the balancing chain concept as distinct from any mechanisms that attempt to restore equilibrium by dynamic means. Yet in biology, Cannon clearly understood even another physical distinction. In naming homeostasis he says quite precisely that he wanted to distinguish a mechanism for steady state constancy - namely homeostatic or regulating mechanisms - from mere equilibrium or force balancing mechanisms. The third class of mechanisms, not included, are control mechanisms. All three types are included in a theory of coupled networks.

4. The central principle of linear servo theory is the utilization of the error function. The central principle of cybernetics is not so clear, except to vaguely suggest that feedback, as in linear servos, is a useful concept.

5. A circular response is not evidence of 'animate' behavior, but of a differential equation. If biological authors want to marvel, they should marvel at the active network concept, as even more marvelously the active network involving amplification. It is this property which has been included in servo definitions and that make it unique from other systems, although the mathematical theory appears very similar. In a servo, there is a power amplification from one small electrical signal to the large mechanical power system. Here lies 'accuracy'.

6. The limited response noted in human behavior is not 'proof' of controllers, but as discussed in the first report, more likely of control mode behavior.

7. The question of inferring structure from response is only unique in the linear case under certain restrictive conditions that network theory has developed. It is not possible to infer structure from input-output in non-linear systems, except by a very lucky and knowledgeable art. For example, one minor case was recently completed on the 'laws' of addition of radiation insult to a biological species (16). Physiologists over the next ten years will demonstrate whether the inferences in that study were right or wrong.

8. If not servo theory, elementary regulator theory, elementary control theory, what then? One whole intent of this study has been to do enough examination of biological information to bound the answer. It appears to lie in the domain of non-linear mechanical theory, in particular in the stability associated with non-linear limit cycle oscillator systems. To claim any knowledgeability here, one must at least be familiar with sources like Whittaker (3) and Minorsky (15)).

Rapoport then examines a second scientific methodology, information theory, which he says is allied in many ways to cybernetics, "but even further removed from classical physical science". (If this statement is meant to imply that so-called servo theory is far-removed from classicl physics, 
this is quite wrong. The subject is very allied to what is taught in a sophomore course in electricity and magnetism, and no good student, with electricity and magnetism and mechanics, has or would have any difficulty with any of the standard texts in servo theory.) Information theory is identified as an outgrowth of communications theory, and not from mechanics and thermodynamics. (This is not in accord with the course work after the $20^{\prime} \mathrm{s}$ in engineering schools. While mechanical engineering was taught around a strong core of mechanics, heat and power, electrical engineering gradually developed communications theory in addition to power. However, communications theory was laid on one foundation of electrical network theory - a perfectly sound AC power theory developed within the scope of thermodymamics. Information theory itself arose out of telephonic electromechanical switching and the characteristics of electronic switching; for example, in such basic bistable circuits as the Hull dynatron oscillator (1918), the Eccles-Jordan bistable amplifier circuit (1919), the Abraham-Block multivibrator (1919), the Turner push-pul1 negative-resistance oscillator (1920), the Van der Pol uscillator (1926), etc., which indicate the age of the art. Information theory in the more limited sense is contained in such work as Shannon's and Russian work.)

Instead of being concerned with energy transformation, information theory is concerned with machines involving the transfer of information. Telegraphy, telephony, radio and television are cited. (Acturally it was Bell Laboratories, telephony, long distance telephoning, computers, and television that were likely most instrumental in the development needs for a theory of information.) Another view of the nervous system is thus as a telecommunications device. The hope is that this theory will prove of value in constructing theories of nervous function and ultimately of behavior (Unless secret, or done very recently, it is our impression that no communications engineer, no computer engineer, no information switching theory engineer has felt competent to attempt to explain a theory of behavior. It is clear that considerable effort is going into this in the 'bionics' programs. Groups like Bell Laboratories have been involved in such primitives as attempting modeling of the neuron.)

An example is given of a use of communications theory by Mackay and McCulloch, comparing the relative utility of $\mathrm{f}-\mathrm{m}$ and $\mathrm{a}-\mathrm{m}$ in synaptic transmission, where $\mathrm{f}-\mathrm{m}$ is shown to have theoretically more advantage. Other examples are alluded to in treating the system as a channel and relating information rate to the subject's reaction time. Conclusions from such gross behavioral experiments to the detailed structure of the nervous system are impossible. (Here we disagree. It is precisely with an overall mode1ling guide, which is not yet developed enough for the problem; the use and development of the best physical techniques including non-linear mechanics, which is not yet developed enough for the problem; the use of communications theory as a mathematical-logical system, which is also not yet developed enough for the problem; and with the use of neurophysiology and neurochemistry, which is also not yet developed enough for the problem that the problem of mind will be transformed in scope to a soluble problem. Accepting the premise that a11 of behavior and the governed system consists of and is governed by an interlocking sequence of oscillators, that this mathematicallogical-physical thesis is quite similar to the Freudian psychoanalytic thesis and with the present state of knowledge in these pertinent fields, much of the basic problem of mind can be untangled in a very few years.) 
Rapoport then discusses applications of a theory of automata, "a class of machines to which the high-speed computers ('giant brains') belong". (It would have been more significant to have referred to a theory of logical machines, and based it on the work of Babbage, Turing, Post, Gödel, and von Neumann. Else the more elementary nature of the present generator of computers never becomes clear. By no stretch of the imagination, except in popular writing or in fanciful proposals, do current computors become 'giant brains'.) "There is no question, of course, that in some respects automata behave as thinking beings". (There is considerable question that they do so in any significant way.) McCulloch and Pitts have shown that certain logical systems can be constructed consistent with a model of neuron interaction. In fact automata bchavior has bcen shown to be capable of greater flexibility than has been imaged. (By whom? The author illustrates with a simple machine algorithm and regards this as making decisions. He illustrates a simple wordy proposition and regards it as illustrative of somewhat complicated thinking. However, the real problems are such logical difficulties as have been cast by Gödel's work, illustrated by a remark taken from Nagel and Newmann (63) who state, "Gödel showed that the axiomatic method, which mathematicians have been exploiting with increasing power possesses certain inherent limitations when it is applied to sufficiently complex systems - indeed, when it is applied even to relatively simple systems such as the familiar arithmetic of cardinal numbers". Yet any mathematical physicist, or indeed any other scientist who has tussled art - like with the tough problems of science, with no possible axiomatic foundation, and in fact with no axiomatic foundation for his entire scientific methodology, will find it very hard to accept that the simple axiomatically devised 'idiot machine' will solve such problems. The idiot arithmetic of quite well conceived problems, yet; the logic of difficult to conceive of concepts, no. Only the brain, as computer, is sufficient to encompass these problems. Current generation of idiot machine automata are not.)

Arguments rage over whether computers really think, but this is fruitless without a definition of thinking. What automata theory has shown is having described the thinking process, an automaton can be built to duplicate it. (This is naive argument. What was mentioned before was that even arithmetic is not closed, nor is thinking. Expecting a 'definition' of thinking to include all of its own operations without an infinite regression of meta concepts is naive. It is unlikely that a thinking can be described by a fixed axiomatic structure. It is likely that the brain is flexible enough to manufacture logics - 'Hey, call me a taxi!' 'O.K., you're a taxi!' logics if you will - as it has to. This is not the present generation of computer logics.)

Hassenstein and Reichhardt have studied beetle eye facet response with a McCulloch-Pitts scheme, but their model is essentially descriptive rather than theoretic. The 'Iifelike' character of automata equipped with servos and one or two 'motivation' mechanisms has been demonstrated in amusing physical models. (Unfortunately, these and checker and chess playing, and gambling machines and other closed large numbers games all tend to confuse the issues. It is not that strategies don't have a point, even to lend descrip- 
tion to mental processes. However, they have to tend to be open, more like so-called modern Bayesian strategies, which require judgment from point to point to improve the process.)

Rapoport then discusses theories of neural nets. The weakness in automata theory is its fixed (axiomatic) behavioral patterns, whereas the living brain and its neural units adapt and are plastic. Particularly, simpler brains do not seem specific but more general, opposite from what one would expect from automata. Further, living behavior is approximate, almost probabalistic, grasping at gross aspects. (These comments are much better.) A first model requirement thus turns to a theory of the structure of the random neuron net. It would then be possible to add statistical properties to the connections. Ashby's homeostatic models are discussed. Earlier models by Rashevsky, Hebb, Wiener, and Schimbel are also mentioned. (A main element of redundancy which McCulloch validly proposed as an essential ingredient to make the system work is not mentioned. In this section, the author withdraws any prior naivete of axiomatic automata as representative of brain functions, and finally substitutes what is the most modern neurophysiological attempts at brain functions, the logical-physical properties of the neural net. It appears clear that the neural net problem is a matter for detailed study by neurophysiologists working in the laboratory with a few decades of neurological experience at their finger tips. However, an overall theory of the brain is not in the same category. For example, our first contributory question for the neurophysiologist is to figure out where the reverberating chains are, as they are indicated in reflex arcs, how they are grossly formed, i.e. to track their path in space and time, like strokes of lightning, and how they transform and shift in time. The one intuitive clue that can be proposed is that the chain must likely shorten up in time to approach a 0.1 second total transit time that it finds necessary to store in the system. The apt analogue is not electrical networks, but the river network (62).)

Rapoport then examines the value of these ideas for behavior. The temptation to plug in these concepts is great. At least, there is a temptation to use a general theory of organization, a general system theory, for al1 systems other than physical. It is axiomatic that generalization represents a chance for progress, but there exists dangers. (Very good. All good scientists lay down these cautions. However, in the present case, we do not believe that the author's concepts offer the needed science for behavior, whereas we believe that this report.has now laid down such a general principle.) He proposes two flagrant examples. Homeostasis is described as a general systems principle par excellence in physiology, ecology, economics, physical systems, etc. However, when the operational meanings of the variables is lost sight of the language, at best, becomes metaphorica 1 , with no theoretical significance. The reader and writer give the illusory impression that the phenomena are understood. (Very good. By thus saying so, the author has drawn the issue involved. We do not agree with his discussion off homeostasis, cybernetics, servo theory, or feedback control theory. Rapoport states, "The concept of homeostasis was formulated in the context of physiological regulation...". This is true. "In such regulation homeostatic mechanisms operate so as to keep certain variables...within certain limits of tolerance". This is also true. "Obviously homeostatis is also a central 
principle of cybernetics...". This is not true. "...the regulation of behavior of servo mechanisms can be described in very much the same terms as the regulation of the living processes". Perhaps, "In general, homeostasis operates on the principle of feedback". Not true. "In negative feedback, the "restoring force"...is always opposite to the error..." etc. True. "Homeostasis ...is attained by a proper arrangement of feedback loop". Not true.

Cannon clearly reserved the idea of homeostasis for something other than a simple end point 'equilibrium'. He states so as his reason for naming the process homeostasis rather than equilibrium. However, he also clearly states that homeostasis is to be viewed when all other disturbances haveceased. Thue he was not proposing a dynamic maintenance of level, but an ultimate static return to a maintained leve1. Starting from Watt in the steam engine and Maxwe11, the concept of regulation or governing of level had long been known and accepted mechanically. Pressure regulators, voltage regulators, speed regulators, etc. are all well known engineering elements. They do not perform their function dynamically. The physical ideas that are generally involved are a force balance that holds this near constant level in the force of static disturbances. Cannon implied this in all of his examples. That he wanted to coin a term homeostasis for physiological regulators is perfectly satisfactory, as good as any other 'trade' name. However, cybernetics, servos, and feedback circuits are al1 conceived of for control during dynamic disturbances, and are thus not the same as homeostatic mechanisms. Wiener may have wanted to include regulators in with controllers under a common roof of feedback, but calling a horse a cow doesn't make it one. Thus the mish-mash of apparent physical or engineering theory without specific applicability is a point well taken, but the article is itself thus guilty.)

The second apt flagrant example is the information-entropy metaphor. A link between thermodynamics and information theory has been tantalizing to biology. Meyerhoff and Schrödinger have left the idea that organisms can create order internally; i.e., reduce their entropy; but this can only be done without violation to the second law of thermodynamics. The work of Wiener and Shannon in cybernetics and information theory have connected information and thermodynamics, which Szilard has given determinate physical meaning. This is being applied now on the molecular biological level. It is often misapplied on gross microscopic levels where 'information' is not validly understood. (Valid connection between information and theremodynamics can only exist when a statistical mechanical foundation is aptly cast. When one deals with physical molecules, the foundation for thermodynamics is there. We would not propose to begin a long review of what it takes to establish a thermodynamics of a system. The interested reader is referred to source material in the statistical mechanics or statistical thermodynamics of irreversible processes. The briefest path is by summary works of Tolman, Fowler, Hirschfelder et al, Rossini. What it takes to get a set of equations that can describe both mechanical and thermodynamic events in a system is illustrated for the hydrodynamic field in moderately irreversible conditions in a recent review (10). Thus the reference to 'thermodynamics', while sporting, doesn't contribute much.)

In summary, Rapoport points out that physical science has both nourished 
the life sciences in the past with regard to matter and energy and now does so for organization. Mathematical physics and logic can present methods. However, their value to the life sciences remains open to question. The uncertainty principle is cited as an example of a physical principle quickly generalized philosophically, with false implications such as free will. Yet psychology seems to demonstrate such a principle. How does one formulate the real analogue? While ideas are fruitful for suggesting parallellisms, there is considerable promiscuity in behavioral scientists in borrowing language of modern physical science, a process most distasteful to physical scientists. (Amen!), who tend to adapt common words to highly technical usage. Beware. Transplant with care. Be sure that the soil is ready to receive the transplants. (We are in complete sympathy philosophically with Rapoport, but he is guilty of what he warns against. A number of physical methodologies, which have generally had very highly technical usage and application, have become popularly summarized in elementary fashion, and then the elementary summaries have been transferred to other fields without the slightest chance of achieving any detailed successes. Without any doubt, these translations stimulate imagination by analog, but the long range progress is beclouded. Our point of view is to apply theoretical and cxperimental tools to a variety of 'physica1' problems, and against the popular direction of reaching to the 'fundamental' problems of modern physics, to stick to applied physical problems with a classical physical apparatus until they are solved. Biophysics is a case in point. Others may consider molecular biology, quantum chemistry, etc. the most apt fields for physics in biology; we see the 'mundane' application of laws of mechanics, thermodynamics, electricity, etc. to the problem of physical modelling of biological systems and subsystems. The main tool is the laws and methods of classical physics, and the scientific method developed in physics over the past 300 years. The heart of most problems becomes a spectfic exercise in non-linear mechanics which is still not a well developed science but a very skilled art. (In 'discovering' Newtonian mechanics in every 'atom' of human behavior, one can only feel like Freud in discovering sex.)

For a near final review it was apt to glance at a relatively recent book by Scher on THEORIES OF THE MIND (64). Just a few highlights will be touched on.

Preface. Understanding the nature of mind itself is st111 a direction, not yet a goal, for probably mind can never completely encompass itself. (It is this attitude that must be challenged). A truer view of brain functioning complexity both inspires and intimidates. The pure scientist, if he is dubious of philosophy, should start with the essay of Rapoport, a serious attempt to define terms and dimensions acceptable to scientist and philosopher. Yet like the enigmatic elephant, mind still remains unknown in size and coordination, both sma11 bits and guesses at the whole are valuable. (True, and like one of these fabled observers, our report proclaims a guess at the whole, as hypothesis.)

Bailey - "Cortex and Mind". In evolutionary view, mental operations must derive from ordinary physical principles by progressive steps. Signal 
handing in the brain has not been understood by any analogue with fixed machines including telephone systems (switching networks). But with the electronic switch, it has been possible to construct machines, computers, that have brain-like properties - seek goals, learn, forget, etc. (This is not an impressive idea, for a theory of switching whether telephonic or electronic is essentially equivalent, and both are really subject to physical limitations, not as free as many might think an information theory to be. As Wiener himself pointed out, it is the cheap, fast, simple nature of the switching element with a large memory storage that made the computer possible. Is this the case in the brain? It represents much of the reason for current search, say particularly, by Bell Laboratories). The cerebral cortex acts similar to a computing machine, correcting error by negative feedback. The mechanism has long been known to physiologist and psychologist, only the name feedback, borrowed from engineering is new. (For the past decade, we have been against adopting this idea and term as being a useless oversimplification for psychological purposes. This intuitive supported fight has now bloomed forth with a countering idea.) It means that receptor and effector can each stimulate each other. (This only means coupling). With the external world, the cortex formed a dynamic system in stable equilibrium which tends to improve its stability against disturbance. Cortex structure is compatible with a feedback analog because of its vast random neural nets and self-reexciting chains between input and output. Ideas about cortex structure were long confused, through Gal1's influence, as a mosaic of organs, of delimited regions of special structure, implying difference in function. This was encouraged by finding areas involved in vision, with muscles, with auditory impulses, etc. However, the greater part of the cortex was found to have a 6-layered very similar structure. (The first note of a theme begins to arise, that just as we had to return the focus from Womersley and pulsatile events back to Frank and windkessel in the heart; from wiener and cybernetics back to Adrian and direct neural impulses, plus Freud and the erotic impulse in the mind; from Hardy and control back to Lavoisier and Rubner and thermal energetics in the temperature control system, each time noting the newer ideas and attempting to regraft them onto the old, here again it is likely the old that has the better case. However, the structure is not necessarily fixed in the mind, but borrowing from the already existing nomenclature, reverberates around the existing structure like the analogous memory delay line that people probably recognize. However, different from the delay line more like the river network, the structure is fastened during 'geological' epochs, and is not a simple energetic signal. The need will be, it is guessed, for estimating the extent of the oscillator cycle elements. It is in these oscillator cycle elements that a structure of 'computer' logic other than a Boolean algebra is built up. Warren McCulloch sought the logic in the neural net, but couldn't quite find it in satisfactory complete form. We propose that it must lie in the functioning of the oscillator cycle complex.) As a communicating machine, the cortex must be largely determined by extrinsic connections. (No, not if internal oscillators. External connections may only affect internal stability). Thus isocortex may be understood as a machine for handing signals, many inputs being known, and with a fixed mosaic of organization, but the vast remainder is a myriad of nerve nets, capable of infinite connections "so that its patterns are dynamic and fluctuating". (We always find 
what we want, this is a very good way to have organized a system capable of self-organizing internal oscillators. It is still not clear how to prevent it from becoming unstable). The cortex, starting with nets mainly randomly organized, modifies the structure. Many parts interact after the manner of feedback. It has been abundantly demonstrated that the cortex nature is dynamic, and by altering feedback in the cortex there can arise serious perturbations including epileptic attack. (As the references become more nearly neurophysiological, one begins to note that the comments become more specific. One would suspect what is easily recognized as 'dynamic' is oscillatory behavior. Thus it is more pertinent to note that feedback means coupling, and that the coupling effects are likely ones that affect oscillatory stablitty, i.e. shift frequency, amplitude, or in these plastic systems, shift even the network. To cite an illustration, suppose networks were used with a principle of least time, to find the connection that can be made most rapidly; or with a principle of constant signal level, to find that path those net amplification gain neither increased or decreased amplitude. Both. of these are hypothetical, but it wouldn't take very many of such principles to lead to a very complicated appearing system.) Though horizontally random, the cortex is vertically organized in 6 layers. This has been viewed as organized as a scanning mechanism. While visual images are significant in mental activity, a great deal is internal conversation; both are characteristic of cortical areas. Ashby's homeostat indicated analogue network capable of rearranging its own wiring to reach new equilibrium. While the cortex is a signal handling machine, it is not the only machine, nor all of the brain. For example, consciousness has been traced from the cortex, down through the basal ganglia, the hypothalamus, and the midbrain into the bulb. The mindbody problem transforms into the mind-cortex problem but leaves a consciousness - brain stem problem. Freudian superego, ego, and id are pseudoscientific terminology. (The neurology is fine, the physical philosophy however leaves much to be desired).

Masserman - "Biodynamics". From observation on animals; lower animals can learn. There are several correlants of instincts and experience. There may be innate learning. Animals can act out solutions. There can be adverse effects from early experiences. Animals show regression. They communicate by various senses. They can use tools. They court. They show aggression, friendship, wooing. Wifely behaviour is shown. Parental conditioning is suggested. Biodynamic 'principles' can be demonstrated; such as behavior is motivated by physiological needs; each animal assesses its milieu in terms of its own experience; when goal direction is blocked, the animal seeks other paths or substitute objectives; if two motivations are in opposition, anxious tension develops, and its behavior becomes either poorly adaptive - neurotic; or symbolic and disorganized - psychotic. There are procedures to ameliorate experimental neuroses: satisfying the need; prolonged rest removed from the situation; forced starvation; reexploring; transference; social therapy; drug therapy; electroshock; neurosurgery. The identity with human clinic experience is not sweeping, but very parallel.

Masserman then discussed psa research. Freudian theory is discussed as being conceptual and not somatic even though it refers to existing organs. (Suppose it were somatic, might there then be real mechanisms that require a 
different formulation than the physiologist and psychologist have so far proposed?)

In current psa treatment, treating young we11-off adults, in half the cases a complete analysis was achieved in about 200 interviews. Of these analyzed cases, $90 \%$ were standard analyses, while $30 \%$ were in doubt of diagnosis. For half the analyses, there was no cure expected for neuroses, character disorders, schizophrenia, schizoid personality, and homosexuality. Improvement was expected in about $50 \%$ of the cases with anxiety; $33 \%$ for dissociation. (Psa has thus succeeded in modelling of mental activity for clinical purposes to a significant extent).

John - "Psycho-physiology of Mind". John indicates a physiological model for discriminative learning. Reality is represented in the sensory cortex; memory is generated in non-specific subcortical structures. A schematic version of the system is indicated, (beyond our current scope to understand or describe meaningfully), but a detailed examination of compatibility with behavioral data is beyond the scope of the paper. While the model has some encouraging capabilities, it has many that are not. One of the difficulties is the problem of input encoding. (It is the use of this type of material and its references as a starting point, namely of detailed neuro-physiology, that must be reviewed for a real start on brain modelling).

Himwich - "Emotional Aspects of Mind". (Another useful source for sumnarizing brain mechanisms, and some electrical responses in various parts of the brain).

Liddel1 - "Biology of 'Prejudiced' Mind". Sufficient repetitions will fix a 'prejudice' for life. All conditioned reflexes are prejudices and therefore neurotic traits. This is reconciliatory of both Freud and Pavlov, in noting that much of their differences results from considering different ages of subject interest. Prejudices formed by Pavlovian conditioning in young animals may be quite disabling. The human baby, fortunately, is emotionally tough.

Rapoport - "An Essay on Mind". (It is appropriate to be able to examine this second article by Rapoport). What is mind in philosophically discussed. (The only comment that will be examined is the following: In discussing Descartes' differentiation between automata and men "The only automata known to Descartes were clockworks. The 'behavior' of a clockwork is entire prescribed. Once it is wound up, its parts go through a predetermined sequence of motions determined by a fixed arrangement of the parts. It is not clear whether Descartes' thought of the behavior of animals in this way. Today we would dismiss such a motion as absurd. A clockwork does not even have inputs in the usual sense, that is, the outside world cannot influence the course of events prescribed by the inner mechanism of a clockwork except to start the mechanism, to stop it, or to destroy it." Yet is is precisely such oscillator mechanisms that are here proposed for the biological system. The rigidity that Rapoport proposes is only true for fixed type of resonator L-C oscillators. The whole point of Van der Pol's developments of the relaxation oscillator was that it can be shifted. Nor is it 
necessary that the $\mathrm{L}$ and $\mathrm{C}$ are fixed even in a resonator oscillator, as every tuned RF oscillator indicates. Thus it appears that our hypothesis has not been clearly foreshowed by any source so far reviewed except, perhaps, by Van der Po1.)

Ashby - "What is Mind?" Understanding of the behavior of mind has been clarified by the digital computer as illustrating a complete logic of mechanism. The concept of mechanism changed with the computer from a metal machine to a general theory. (This is not true. The engineer may have had this view. The physicist - mathematician - logician has seen the problem much differently as the work of Turing and Post have illustrated). The behavior of any machine can be represented on a computer. The statement that a computer can produce every well-defined form of behavior is uncompromising, and must stand unless a specific example to the contrary can be produced. (Fine, then it is desirable to refute this thesis by some examples, such as: If our Fiat is run continuously for the next year, it will fail. How may a computer represent this operationally well-defined behavior? There is a crater on the opposite side of the moon in process of developing. How can the computer represent this behavior? There is 6000 years of economic data available on a variety of civilizations. What is a general theory of economic behavior? The equations of hydrodynamics have been available for quite some time. Can a computer program solutions for turbulence? The same question for the $n$-body problem. There are many 'natural mechanisms' avai1able whose behavior is quite well-defined, although perhaps subject to incomplete specification. Furthermore most real problems lack complete specification. What the human is called upon to do, is solve the problem by induction, intuition, etc. without complete knowledge. Machine-1ike adaption, as for example in so-called adaptive control theory, is still a most trivial problem in programming a given closed class of functions. Human operations may ultimately be explained by suitable generalization of these concepts, but not within the present generation of machines. One can inspect a very recent American translation of a book by Gutenmacher (65), a Russian machine designer, who has been concerned with computer representation of mental functions. The remoteness of being able to deal with any real mental problems would seem to be quite evident, particularly in reviewing the fundamental concepts of Chapter 1 , and the parts therein, such as Section III on an Electronic Information-Logic Machine, as compared with Section IV on The Machine Memory and Human Memory, Section $V$ on Problems for the Machine (analog to the brain), or Section VI on Tests for the Machine (and its accuracy in modelling mental processes). Thus our much earlier statement still stands. It is not yet known what are all the possible responses of non-linear mechanical systems. Modern computers have been built on much simpler logics.) To prove the proposition to the unconvinced reader that the computer can simulate forms of behavior that includes the emotional, say that can produce rhetoric, music, and art, is made intuitively obvious by pointing to the fact that Disney dissects and simulates emotion frame by frame with film frames, black and white, and dye. By these two examples, it is clear that those who think of mind, and those who think cybernetically are approaching the same phenomena with different basic ideas. "Modern studies in the logic of mechanism has shown exhaustively how every concept properly applicable to a dynamic system (whether human, animal, or mechanical) has reference ultimately to various 
ways of behaving." (We do not believe this. We are sympathetic to and impressed with Ashby as a neuropsychiatrist, but we are not convinced. The examples given before had to do with scientific problems. The present group have to do with intellectual, aesthetic problems. Consider first an athletic problem. Given a machine constructed with certain internal operators, can it run a 4 minute mile? If demonstratable, can it run a $31 / 2$ minute mile, etc.? How can one prove this by computer, except to find a progran. The problem boils down here to machines capable of providing borad enough program writing capability. The current concepts of adaptive systems will not do. A Boolean algebra is just not broad enough. The problem illustrated by analogue in another way is that a denumerably infinite set of numbers cannot be used to represent a non-denumberably infinite set. The machine can start to count, but never get there. Aleph null, and Aleph one are just different. One additional pertinent illustration is that the human draws his solution from a hierarchy of types that no one has yet learned how to program. Beethoven can be simulated, ill-tampered pianos can be simulated, Einstein's relativity can be simulated, and if the machine is told what solution to seek, solutions can be simulated, but at present no machine can be simulated with any significant self programming capability except to develop some simple program arrangement. The problem is a logical one of discovery of a sufficiently broad logic. The proposal of coupled oscillators, with the possibilities of shifting equilibria, shifting oscillators, periodic, aperiodic, almost periodic behavior, the conceivability of reconciling a basic question of how 'determinate' equations of motion can describe apparently 'statistically' describable phenomena, are all piled together in the dark corner called 'non-linear mechanics'. This is the proposal which can be made seriously, not because one is sure it will work, but because the system thus appears constituted, and this dark corner has revealed sufficient complexity that it might just be adequate to account for the 'human computer' as opposed to current 'machine computers'. The last question, deterministic versus statistical, is being examined in many places; it is seen in hydrodynamics; it was examined in many places; it is seen in hydrodynamics; it was examined by a great physicist, Jeffries, in geophysics; by Sidney Lees, with a Bayesian point of view, in instrument response.)

Now for the semantic question of mind. Ashby believes that the mind problem - what is mind - results from transforming the adverb, mind, as a measure of how, what, when, or where, into a noun, mind. For such transformations, the object-property must have invariance and localizability in space and time. Sometimes the assumption that a system has a property because it contains a substance can be defended, other times not. In a computer, to invoke a 'substance' for each property would be ridiculous. Thus one must talk about behaviors and not substance. (These scholastic disquisitions are fine for pre-Cartesian /ristotelian argument, and may still be fine in certain fields of philosophy, but Kant, Marx, Darwin, Freud, Russe11, Einstein, Gödel, Heisenberg, Carnap, Bridgman and others have come and gone and made their dialectic mark on the logic of science. The physical scientist we11 understands the problem to be the dynamic systems characteristics of 'mental' functions, and is concerned only with an 'operational' definition of mental functions, and the identification of the behavior with some physical logic that he can comprehend.) However Ashby 
questions whether these same machines, via cybernetics, cast any light on self-awareness, an inseparable part of the whole concept of mind (noun?). Thus in reconsideration of the subjective viewpoint, the machine systems before were discussed from the view of an observer outside of the system. Now a general system theory is considered which observes itself internally. "Such a branch of general system theory has hardly been started", though some have insisted on great importance of this distinction. A 'logical' Instance is given. Its essential feature is that in the prediction illustration discussed, there is feedback. It is intuitively felt that selfawareness has something to do with feedback. There are difficulties contained in such feedback examples, which are simply particular cases of general coupling of an output to an input. "Now the general logic of mechanisms assures us at once that such a coupling will always induce some new characteristic form of behavior." (No!) With the appearance of new behavior, one is puzzled to know whether the degree of feedback shown to exist (in fairly closely coupled feedback systems) is evidence of selfawareness. Yet untutored intuition unhesitatingly supports the belief of a relation between self-awareness and feedback. Mere size, in a cybernetics view, may contribute to the 'emergence of self-consciousness' (shades of Zeno, and Marxian dialectics! A good neurophysiologist thinking about his problems is one thing. We deplore intuitive stumbling around in very specialized mathematical - logical - physical notions and techniques. Apparently most biologists do not think in terms of differential equations with the 'seat of their pants,' and are thus not familiar with what differential equations can and cannot do. General systems behavior - at least physical systems - has dealt with physical systems that may be described by differential equations, most completely, by systems described by continuous, and linear differential equations with constant coefficients. To a moderate extent, explorations have included non-constant coefficients, piece-wise discontinuous functions, moderate non-linear ideas. It has not been able to deal well with hicrarchical questions except in very limited ways. In the first report, the example was given of Ziebolz's 2 time scale model, of process and analogue, intercoupled. This is still today's most complex idea for hierarchical computer control. For the sake of the record, the basic contributions of such men as Ziebolz, C. E. Mason, Minorsky have been made in the mechanical field among mechanical engineers. The point is made, not as a matter of pride, but to indicate that the contributions to this field have and can come from mechanical engineers - oftcn considered backward -, electrical engineers, even chemical engineers, and physicists. As an example of a 'new' idea is the one proposed here both for general systems and the biological system - purely coincidental, because it seems to apply to both - of separate, but coupled, oscillator time scales for a variety of systems that are then loosely coupled by a 'governing' oscillator complex. These ideas flow from and were intrinsically inspired by the background of ideas laid down by such people as Ziebolz and Wicner. However, it would be self deprecating not to recognize a transformation of these ideas through our own experience into a new integration. Thus in our view to try to depict the human for 'intuitive' investigators, as well as other specialists, the human is like an organ.. with a great variety of pipe systems, and internal power systems, and stops, etc. There is in addition an organizing master player, the cortex, that unfolds its own musical story in accord with the surrounding changing environment both past and present, and its own 
internal capabilities. The poet is concerned with the individual content of each organ system. The scientist is concerned with what is common to all such systems in mechanisms and functions.)

Another 400 pages of review wi11 not contribute much more than a selfmessage to go search in neurophysiological and physiological details. (Although it is clear in an article like E. E. Harris, as a philosopher, that there is a greater philosophic sophistication of pertinent background material, including people like Polanyi and Tustin, or in an article like I.J.Good's, than the other writers referenced. Finally, most fascinating was the content of the interpretive interview described by Garfinkel. The human strategy evidenced stands as a good example to make any physical investigator pause to think very seriously of the complexity of behavior that he must account for. The challenge is fairly taken.)

As a final token of what interest will have to turn to in order to apply oscillator concepts meaningfully, it is useful to point toward a neurophysiological article from a recent symposium, by Scheibel and Scheibel (66). In introduction they make the point that the brain investigaor must infer circuit diagrams and operating modes of the brain with a brain, strikingly unable to construct operational hypotheses of lasting value. They then indicate that they chose to look at the cerebral neocortex for insight into information manipulation and command functions, seeming as it does in many respects like a bank of differential analyzers, and then deciding as hypothesis that ultimate command lies in the brain stem reticular core. At the end, finally, they make the point that the continuous signal barrage from the environment is subject to a continuous filtration process in which the reticular activity can deal with signals of importance to the well-being or safety of the organism, or are exotic, by lowering thresholds so that an input ignored 100 times can become important the next; and concluding finally that as the structural and functional properties of the reticular formation increase, it has begun to emerge as the most significant vehicle for control and modulation of the nervous system. It monitors ongoing activity. The total influx integrated at the reticular neuron level becomes an output, reflecting input per unit time not level. (The concepts are in line with those suggesting the role of the phase plane and phase space and other non-linear mechanical techniques as decisive in deciphering performance.) 


\section{BIBLIOGRAPHY}

1. Ibera11, A., Cardon, S. Ann. N.Y. Acad. Sci. 117, 445, 1964.

2. Ibera11, A., Cardon, S. NASA Contractor Report CR-141, January 1965.

3. Whittaker, E. T. ANALYTICAL DYNAMICS. Dover Press, 1944.

4. Ibera11, A. S. ASME Series D, 1960 Journa1 Basic Engineering. Vo1. 82(1), p. 96-112 (Parts 1-2); Vol. 82(3), September 1960, p. 513, (Part 3).

5. Iberal1, A. S. "A Philosophy For Mid-Twentieth Century Man." February 1959 (unpublished).

6. Ibera11, A. S. "A Study of the Engineering Aspects of Life Sciences For Military Needs." Proposal dated 8-22-60; Life Science Engineering, (4) Quarterly Reports, February 1961-March 1962. Contract No. DA-49$193-\mathrm{MD}-2152$.

7. Iberal1, A. S. "A Physical Critique of the Description of Thermoregulation in the Human." Digest of the Fourth International Conference on Medical Electronics. New York. July 1961.

8. Goodman, L. "On the Dynamics of Pulmonary Ventilation in Quiescent Man." Case Institute Systems Center, Report No. SRC-18-L-62-1, May 1962. Cleveland, Ohio

9. Iberal1, A. S. "Study of Electronic Timer." Report to Diamond Ordnance Fuze Laboratory, Washington, D.C. February 1962. Contract No. DA-49186-ORD-1052.

10. Iberal1, A. S. "Contributions Toward Solutions of the Equations of Hydrodynamics - Part A. The Continuum Limitations of Fluid Dynamics." Office of Naval Research. December 1963. Contract No. Nonr-34-5(00).

11. Andronow, A. S., Chaikin, C. E. THEORY OF OSCILIATIONS. Princeton University Press. New Jersey, 1949.

12. Forsyth, A. R. THEORY OF DIFFERENTIAL EQUATIONS. Vols. I, II, III, IV. Dover, 1959.

13. Ince, E. L. ORDINARY DIFFERENTIAL EQUATIONS. Dover, 1959.

14. Poole, E. A. C. INTRODUCTION TO THE THEORY OF LINEAR DIFFERENTIAL EQUATIONS • Dover, 1960.

15. Minorsky, N. NON-LINEAR OSCILLATIONS. Van Nostrand. New York, 1962. 
16. Ibera11, A. S. First Quarterly Report on Mathematical Modelling For the Mortality Effects of Various Radiation Dosage Schedules dated July 1963; Second Quarterly Report dated August 1963; Third \& Final Report dated February 1964. Contract No. AT(11د1)-1254. Atomic Energy Commission - Washington, D. C.

17. Tsai, D. H., Cassidy, E. C. "Dynamic Behavior of a Simple Pneumatic Pressure Reducer." ASME Paper No. 60-WA-186, 1960.

18. Chestnut, H., Mayer, R。W. SERVOMECHANISMS \& REGULATING SYSTEM DESIGN 。 Vo1. 1 and 2. John Wiley \& Sons. New York, 1951.

19. Popov, E. P. THE DYNAMICS OF AUTOMATIC CONTROL SYSTEMS. Pergamon Press Ltd. London. Addison-Wesley Publishing Co., Inc., Reading, Mass., 1962. (Translation of a 1956 work.)

20. Automatic \& Remote Contro1 - Proceedings of the First Internationa1 Congress of the International Federation of Automatic Control. Moscow, 1960. Vols. I, II, III, IV. Butterworths, 1961.

21. 1962 Joint Automatic Control Conference. New York, Tune 27-29, 1962. Host society and publisher Anerican Institute of Electrical Engineers. New York.

22. 1963 Joint Automatic Control Conference. Minneapolis, Minn., June 1921, 1963. Host society and publisher American Institute of Chemical Engineers. New York.

23. Den Hartog, J. P. MECHANICAL VIBRATIONS. McGraw-Hil1, 1947.

24. Lees, S. "Design Basis For Multiloop Positional Servomechanisms." Dec. 1965. Report 非-96 Instrumentation Laboratory M.I.T.

25. Clegg, B. R. "A Two-Sensor, Lumped Parameter Model of Human Respiratory Regulation." Case Institute of Technology. December 1963.

26. Gray, J. S. AAF School of Aviation Medicine Report No. 310(1), August 1944. Science, Vol. 103, p.739, 1946. AAF Schoo1 of Aviation Medicine Report No. 386(1), May 1945.

Gray, J. S. PULMONARY VENTILATION AND ITS PHYSIOLOGICAL REGULATION。 Thomas, Illinois. 1950.

27. Grodins, F. S. "Analysis of Factors Concerned in Regulation of Breathing in Exercise." Physiological Reviews, Vol. 30. 1950.

Grodins, F. S. et al "Respiratory Responses to $\mathrm{CO}_{2}$ Inhalation. A Theoretical Study of a Non-Linear Biological Regulation." J. of Applied Physiol., July 1954-May 1955. 
28. TEMPERATURE - ITS MEASUREMENTS AND CONTROL IN SCIENCE AND INDUSTRY. American Institute of Physics. Reinhold, 1941.

29. Newburgh, L. H. PHYSIOLOGY OF HEAT REGULATION AND THE SCIENCE OF CLOTHING。W. B. Saunders Co. Philadelphia, 1949.

30. Patel, D. J., Ma1los, A. J., Fry, D. L. "Aortic Mechanics in the Living Dog." J. Appl. Physiol. 16, p. 293. March 1961.

31. Spencer, M. P., Denison, A. B. "The Aortic Flow Pulse as Related to Differential Pressure." Circ. Res., Vol. 4, p. 476. July 1956.

32. Wezler, K., Böger, A. "The Dynamics of the Arterial Systems." Ergebn. Physio1., 41, 329. 1939

33. Hamilton, W., Dow, P. "An Experimental Study of the Standing Waves in the Pulse Propagated Through the Aorta." Am. J. Physiol., Vo1. 125 p. 48-59. 1939 .

34. Alexander, Ro S. "Transformation of the Arterial Pulse Wave Between the Aortic Arch and the Femoral Artery." Am. J. Physio1., Vo1. 158, p. 287. 1949

35. Wetterer, E. "Flow and Pressure in the Arterial System, Their Hemodynamic Relationship, and the Principles of Their Measurement." Minn. Med., Vol. 37, p. 77. February 1954.

36. Peterson, L. "The Dynamics of Pulsatile Blood Flow." Circ. Res., Vol. 11, 非. March 1954.

37. Karreman, G. "The Resonance of the Arterial System." Bull. Math. Biophys., Vo1. 16, p. 159. 1954.

38. Spencer, M. P., Johnston, F. R., Denison, A.B. "Dynamics of the Norma1 Aorta 'Inertiance' and 'Compliance' of the Arterial System Which TransForms the Cardiac Ejection Pulse." Circ. Res., Vol. 6, p.491. July 1958 .

39. Rushmer, R. F. CARDIOVASCULAR DYNAMICS. 2nd Edition. W. B. Saunders Co. Philadelphia, 1961.

40. Spencer, M. P., Greiss, F. C. "Dynamics of Ventricular Ejection." Circ. Res., Vo1. 10, 非, p. 274. March 1962.

41. Greenfie1d, J.C., Pate1, D. M., Barnett, G. P., Fox, S.M. "Evaluation of the Pressure Time Derivative Method for Estimating Peak Blood Flow." Am. Heart J., Vol. 64, No. 1. July 1962.

42. Patel, D. M., DeFreitas, F. M., Greenfield, J.C., Fry, D. L. "Relationship of Radius to Pressure Along the Aorta in Living Dogs." J. App1. Physiol. 18, p. 1111-17. November 1963. 
43. Jennings, F. B. "Theories of Bourdon Tubes." ASME paper 非54-A-168,1954.

44. McDonald, D. A. BLOOD FLOW IN ARTERIES。Arnold, 1960

45. Prescott, J. APPLIED ELASTICITY. Dover Press. 1946

46. Van Citters, R. L. "Longitudinal Waves in the Walls of Fluid-Filled Elastic Tubes." Circ. Res。, Vo1. 8, p. 1145, November 1960 。

47. Remington, J. W., Wood, E. H. "Formation of Peripheral Pulse Contour in Man." J. App1. Physiol.,Vo1. 9, p. 433. 1957.

48. Greenfield, J. C., Patel, D. M., Mallos, A. J., Fry, D. L. "Evaluation of Kolin-type Electromagnetic Flowmeter and the Pressure Gradient Technique." J. Appl. Physiol., Vol. 17, No. 2. March 1962.

49. Levy, M. N., Imperial, E. S., Demattos, A. D., Zieske, H. "Aortic Pressure During Ventricular Ejection as a Determinant of Cardiac Performance." Am. J. Physiol. 205, No. 6, Dec. 1963.

50. Fry, D. L. "The Measurement of Pulsatile Blood Flow by the Computed Pressure Gradient Technique." IRE Trans. on Med. Electronics. Vol. ME-6。 December 1959.

51. Mallos, A. J. "Electronic Caliper for Continuous Measurement of Relative Displacement." J. App1. Physiol., Vol. 17, No. 1, January 1962.

52. Randa11, J. E. "Statistical Properties of Pulsatile Pressure and Flow in the Femoral Artery of the Dog." Circ. Res., Vol. 6, No. 6. November 1958 .

53. Rushmer, R. F., Franklin, D. L., Van Citters, R. L., Smith, O. A. "Changes in Peripheral Blood Flow Distribution in Healthy Dogs。 Part I." Circ. Res., Vol. IX, No. 3. May 1961.

54. Patel, D. J., DeFreitas, F. M., Fry, D. L. "Hydraulic Input Impedance to Aorta and Pulmonary Artery in Dogs." J. App1. Physiol., Vo1. 18, p. 134, No. 1. January 1963.

55. Lewis, T., Lov, W. S. "Vascular Reactions of the Skin to Injury." Heart, $13: 27-60,1926$.

Lewis, T. "Observations Upon the Reactions of the Vessels of the Human Skin to Cold." Heart, 15:177-208. 1929-31.

Lewis, T. "Observations on Some Nornal and Injurious Effects of Cold Upon the Skin and Underlying Tissues." Brit. Med。J. 795-797, 837-835, 869-372. 1941

56. Salter, W. T. A TEXTBOOK OF PHARMACOLOGY。W. B. Saunders Co., Philadelphia 1952. 
57. Goodmain, L. S., Gilman, A. THE PHARMACOLOGICAL BAS IS OF THERAPEUTICS. 2nd Edition. MacMillan Co., New York. 1955.

58. Biderman, A. D., Zimmer, H. THE MANIPULATION OF HUMAN BEHAVIOR. Wiley \& Sons. 1961.

59. Jones, E. THE LIFE AND WORK OF SIGMUND FREUD. Vo1. 2, 1955; Vol. 3, 1957. Basic Books, Inc., New York.

60. FrOIm, E. SIGMUND FREUD'S MISSION, AN ANALYSIS OF HIS PERSONALITY \& INFLUENCE". Grove Press. 1963.

61. Arieti, S. (editor) AMERICAN HANDBOOK OF PSYCHTATRY. Vo1s. 1, 2. Basic Books, Inc. 1959.

62. Iberal1, A. S. "A Study of the Physical Description of the Hydrology of a Large Land Mass Pertinent to Water Supply and Pollution Control." Four (4) quarterly reports dated 1961-62. Contract No. SAph 78640. Div. of iater Supply \& Pollution Control, Dept. of Health, Education, and Welfare.

63. Newnan, R. R. THE WORLD OF MATHEMATICS. Vo1. I. Simon \& Shuster, New York, 1956

64. Scher, J. M. THEORIES OF THE MIND. New York: The Free Press of Glencoe. 1962.

65. Gutennacher, L. I. ELECTRONICS INFORMATION-LOGIC MACHINES E Edited by A. Kent. Wiley \& Sons. 1963

66. Scheibel, M. E., Scheibe1, A. B. "On Neural Mechanisms for SelfKnowledge \& Conunand." 1963 


\section{ACKNOWLEDGMENT}

The authors gratefully acknowledge the technical assis-

tance of R. Ciszak, R. Sedlacek, J. Barco, and A. Williams. 


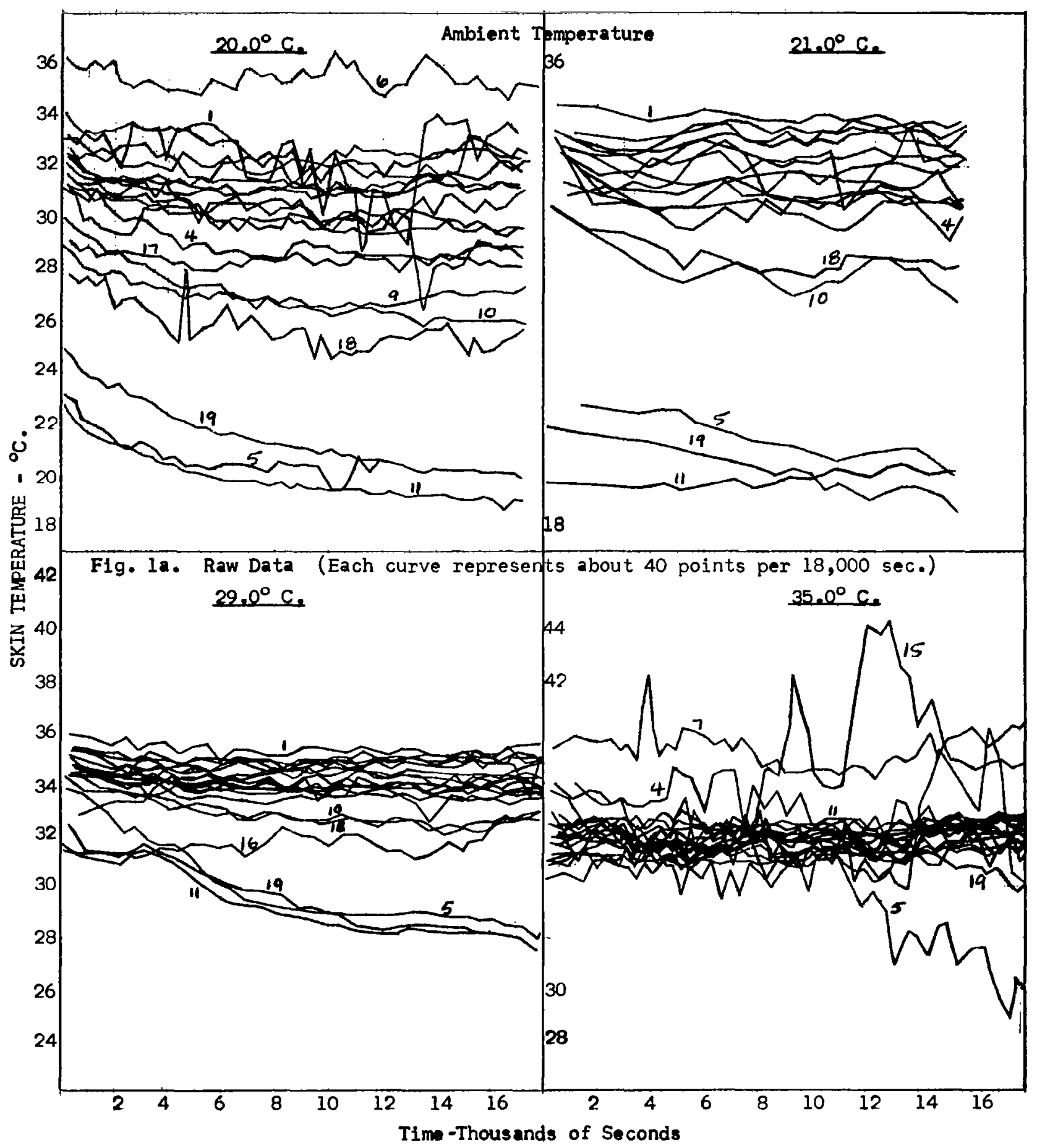

Skin temperature data for approximately twenty body stations on a supine human subject previously described 


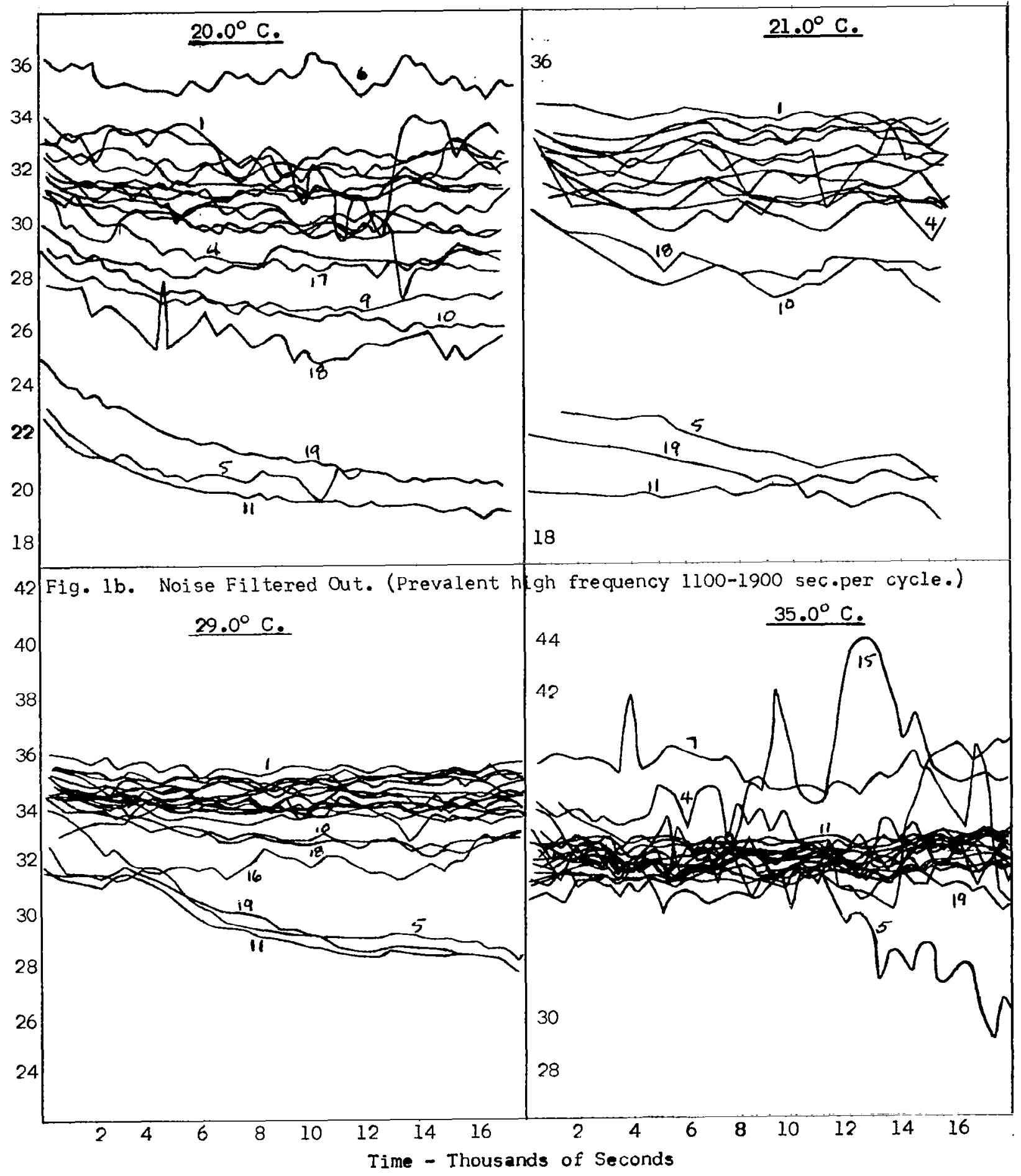

Skin temperature data for approximately twenty body stations on a supine human subject previously described 

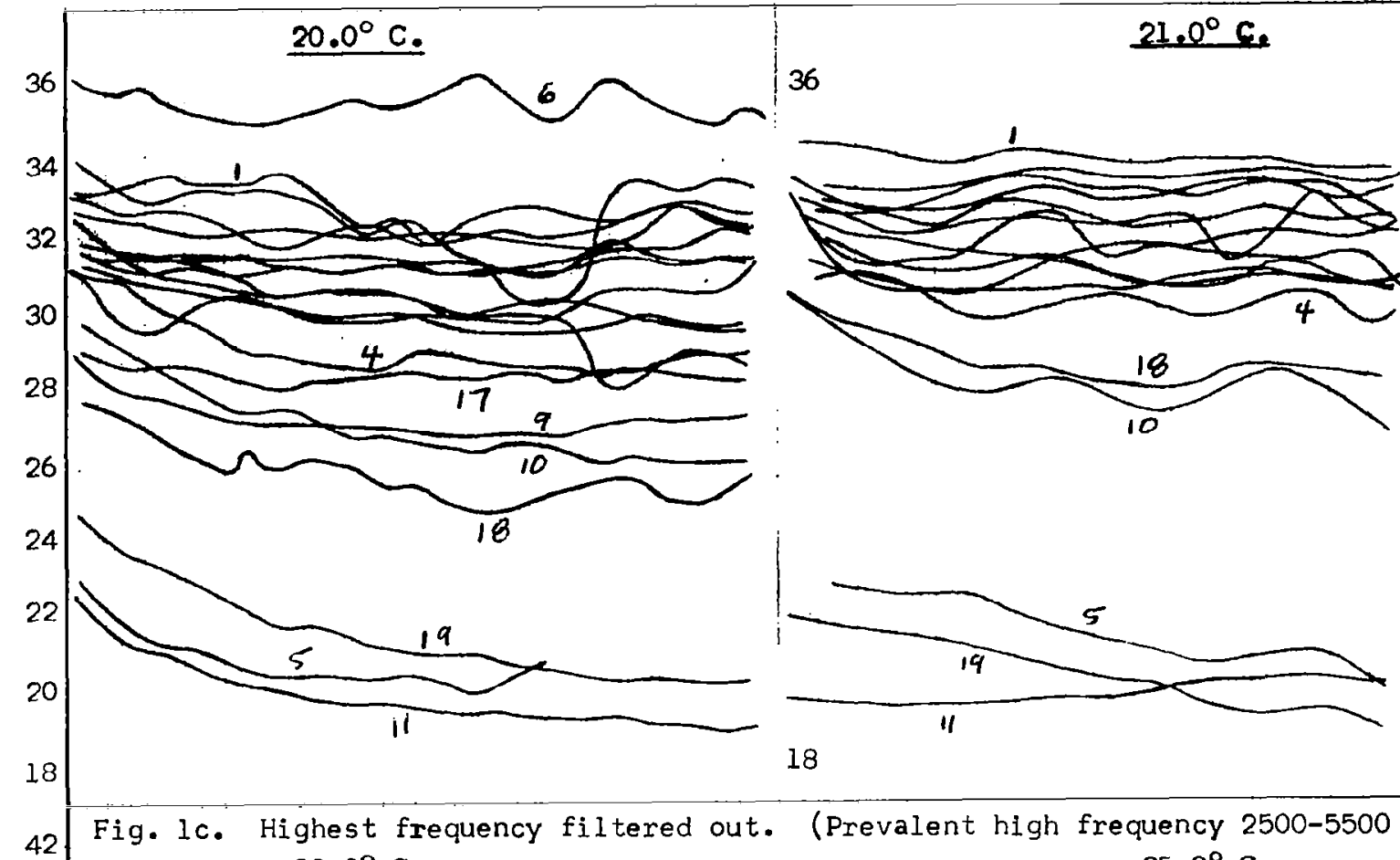

18

40

40 $29.0^{\circ} \mathrm{C}$. 


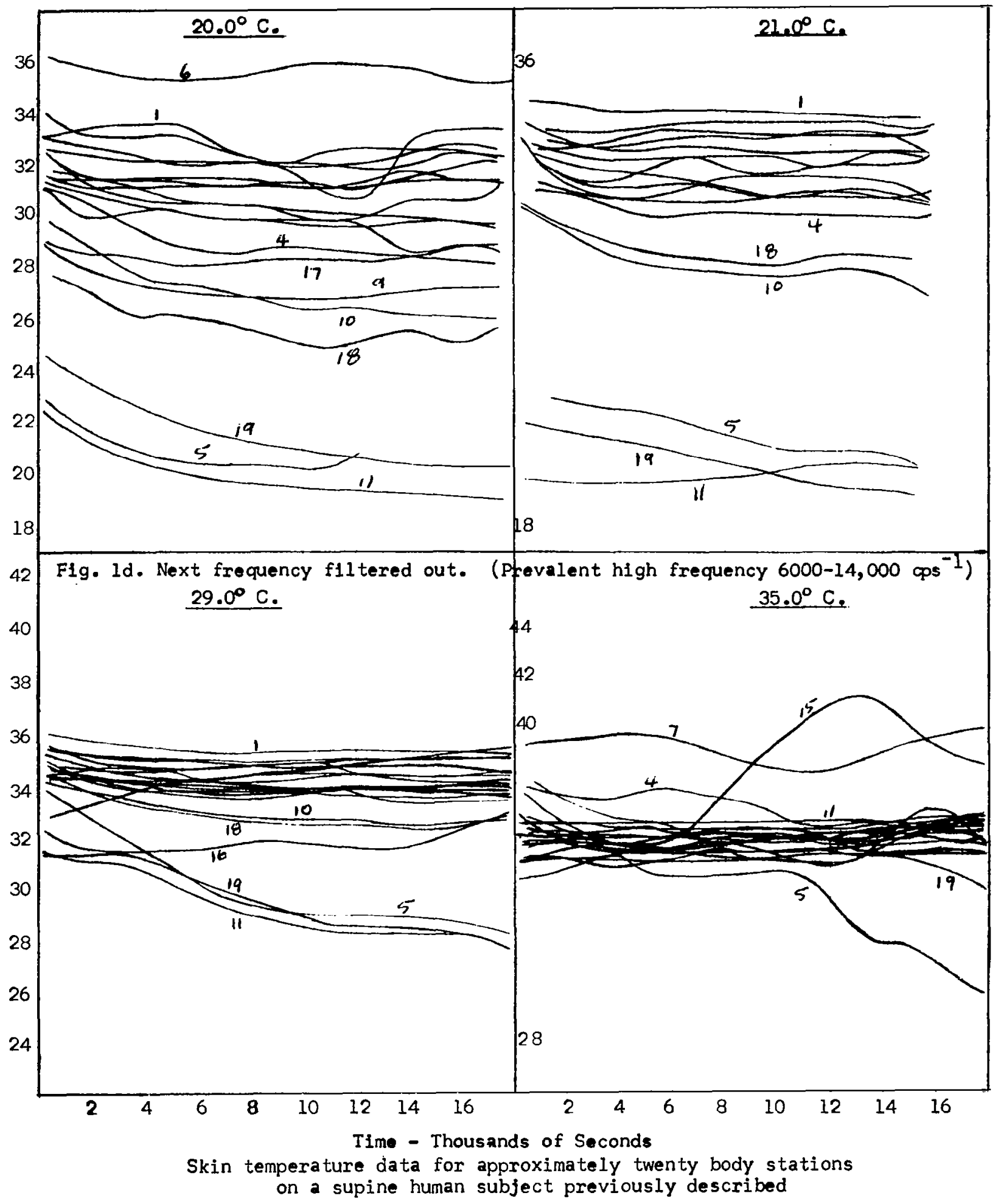




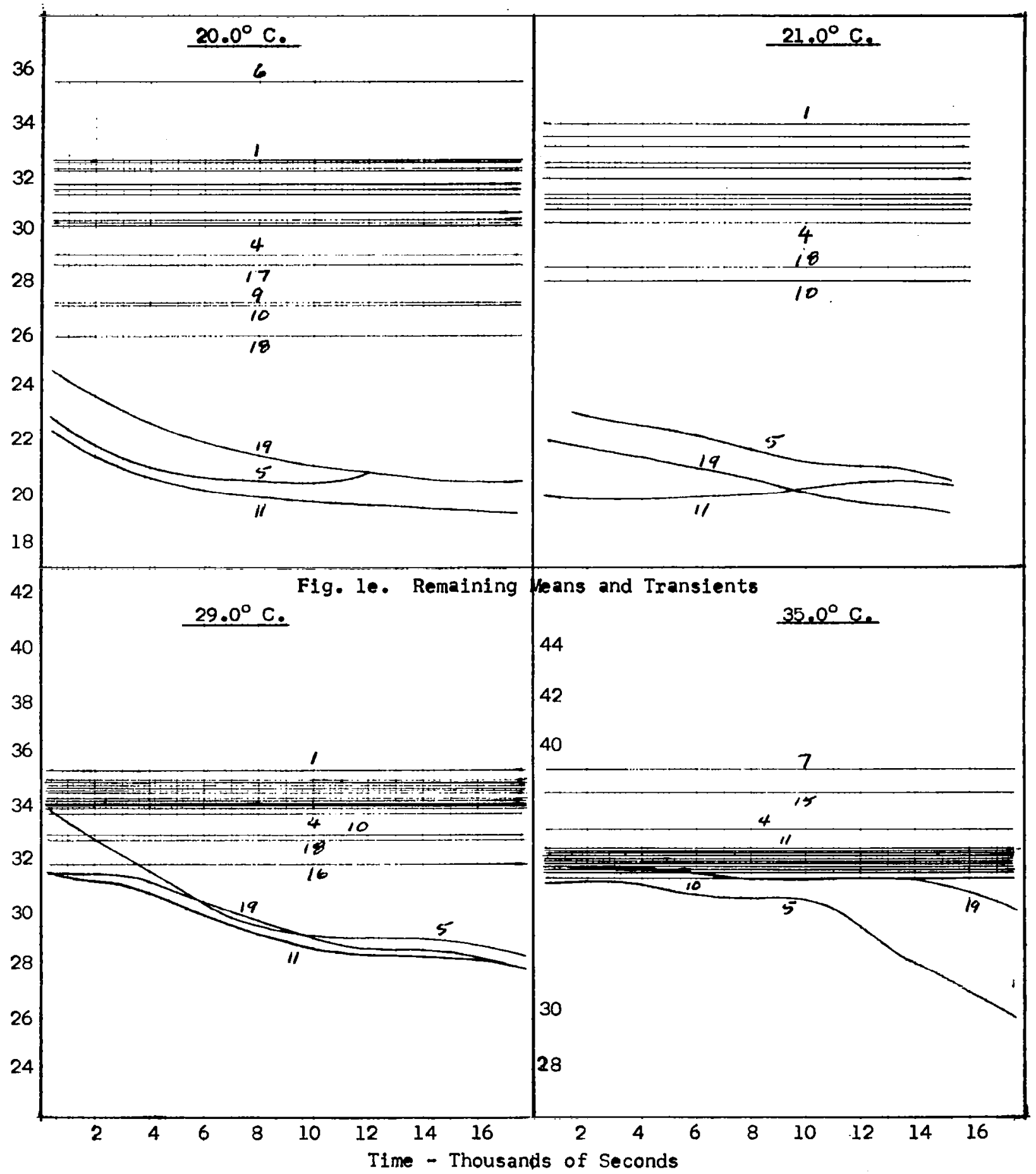

Skin temperature data for approximately twenty body stations on a supine human subject previously described 
Time - Thousands of Seconds

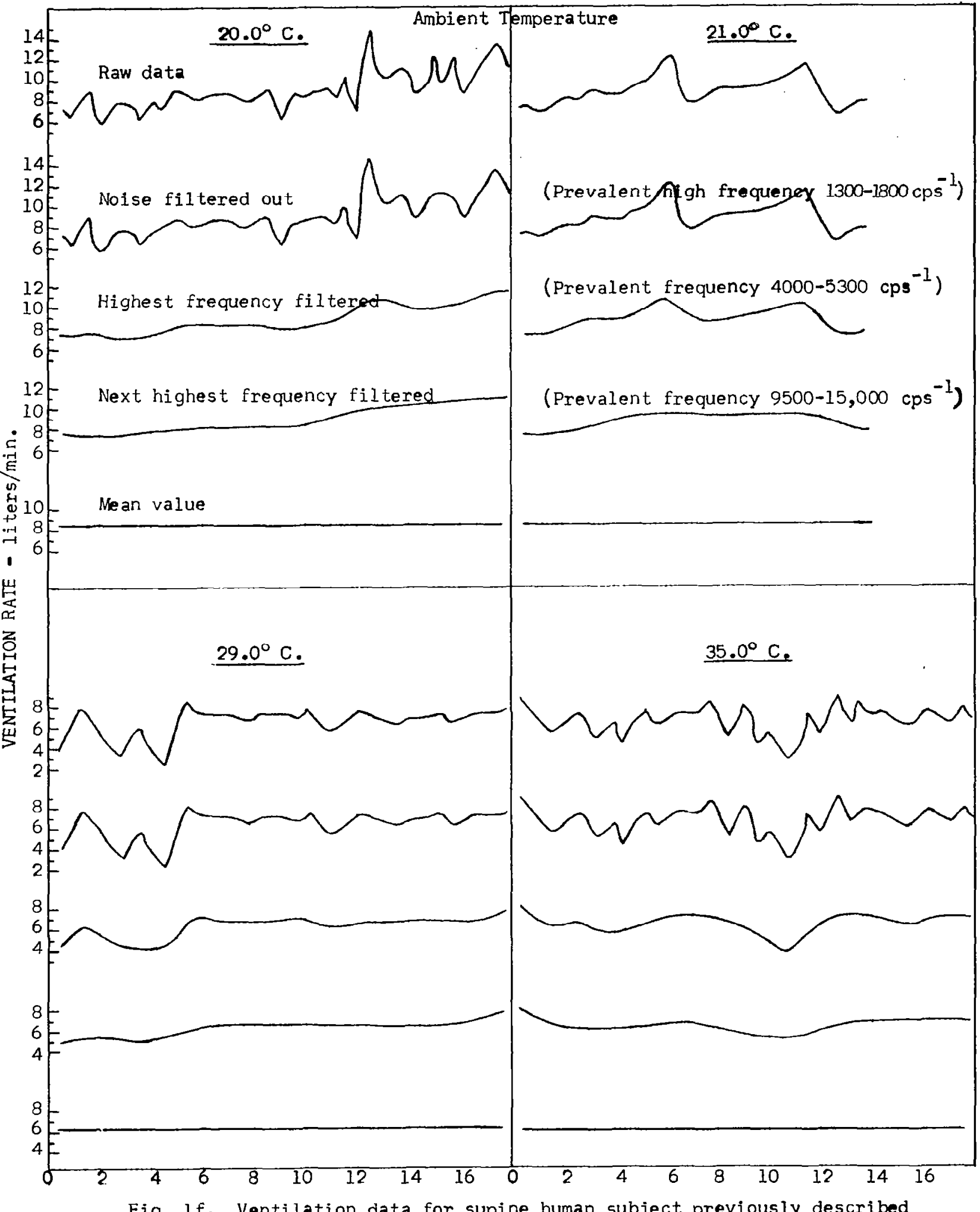

Fig. If. Ventilation data for supine human subject previously described 
Fig. lg. High frequency skin temperature data for supine human subject previously described. (Fast data at indicated stations)
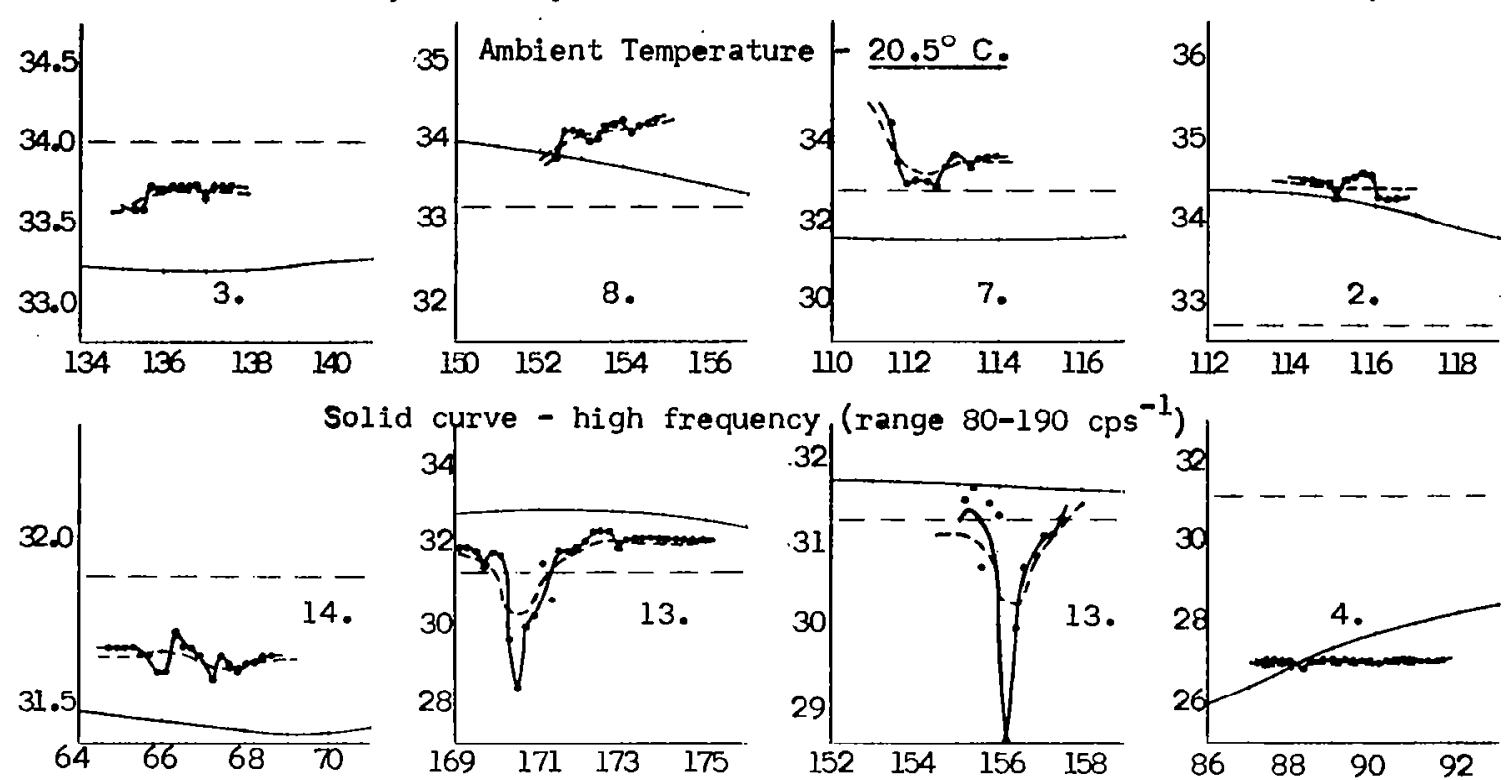

Solid
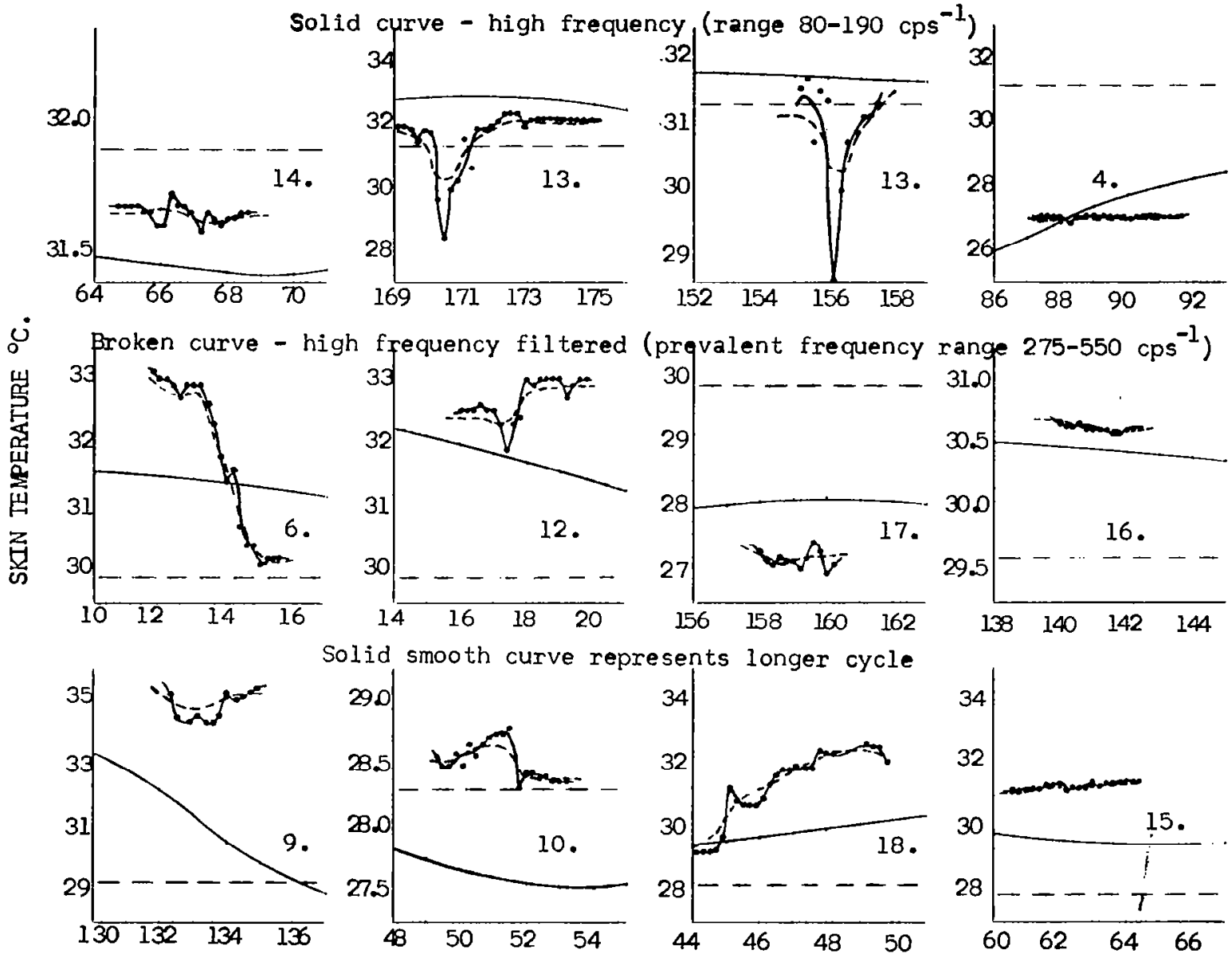
Broken line represents mean value or transient temperature

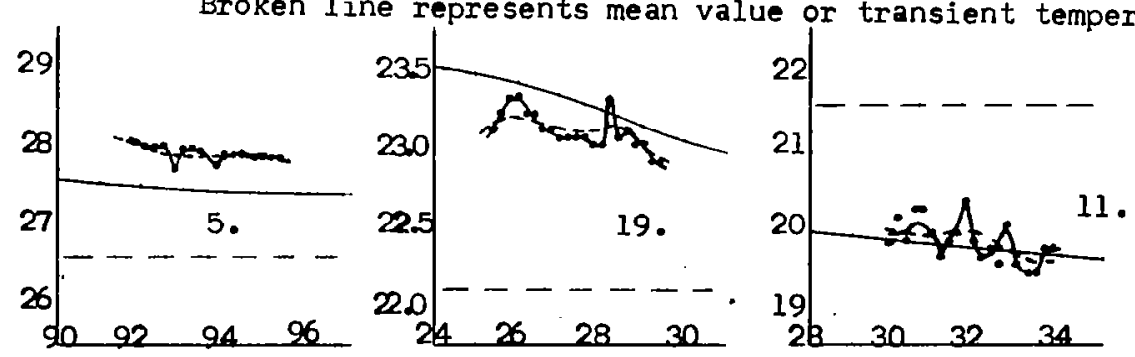

Chronological time scale at indicated hundreds of seconds 
Fig. Ih, High frequency skin temperature data continued
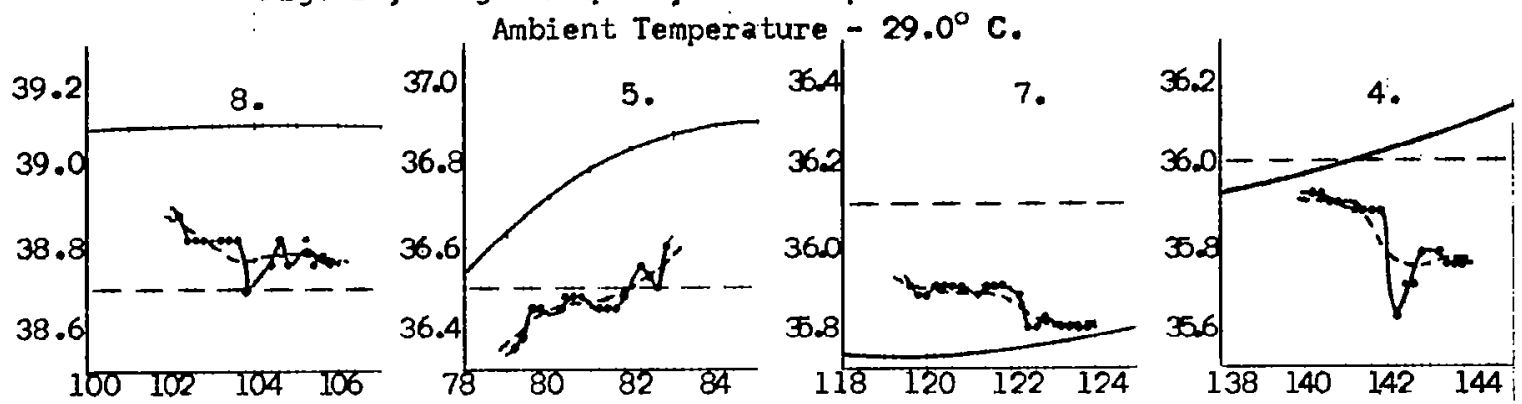

Solid curve - high frequency (range 80-190 $\mathrm{cps}^{-1}$ )
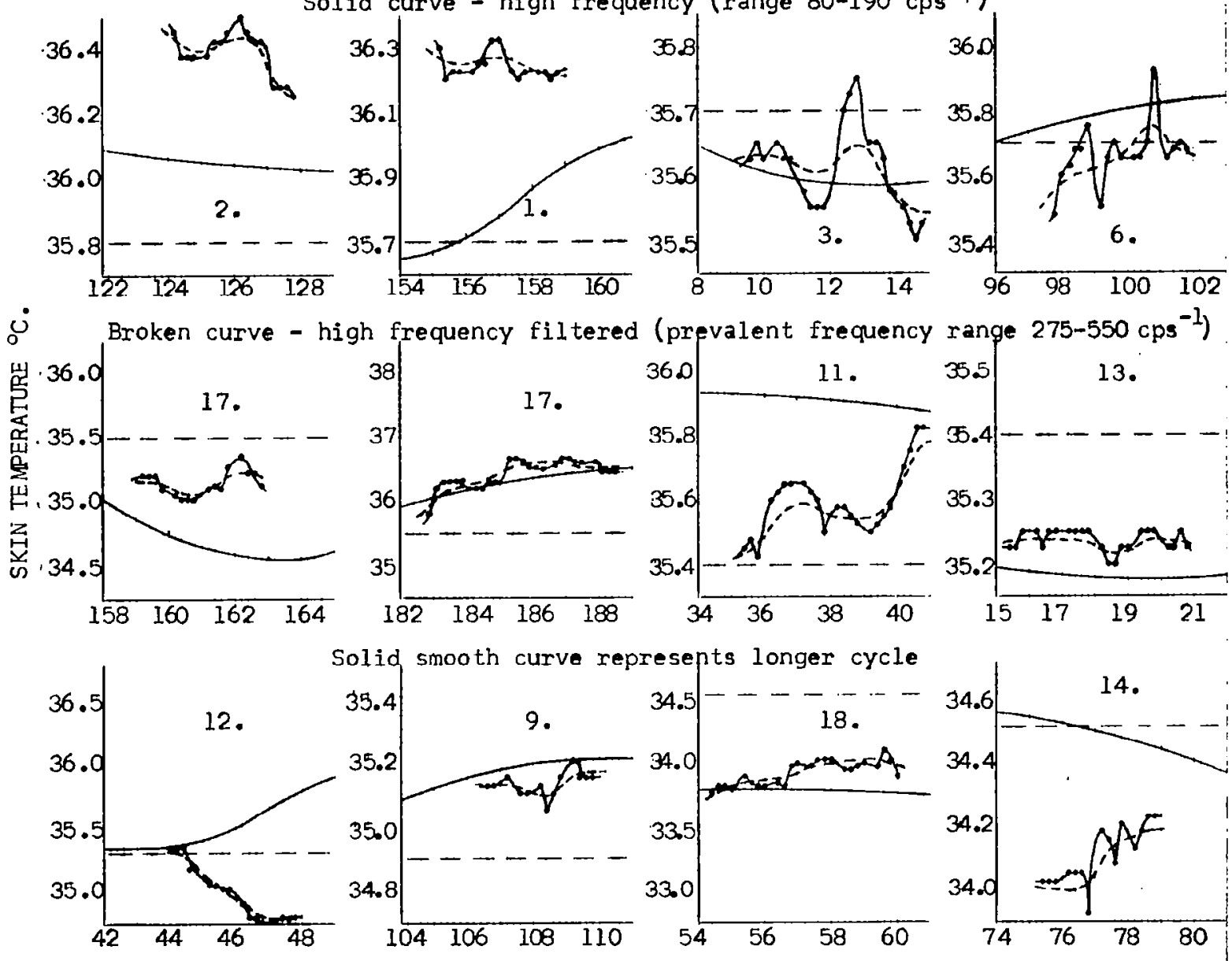

Solid smooth curve represepts longer cycle
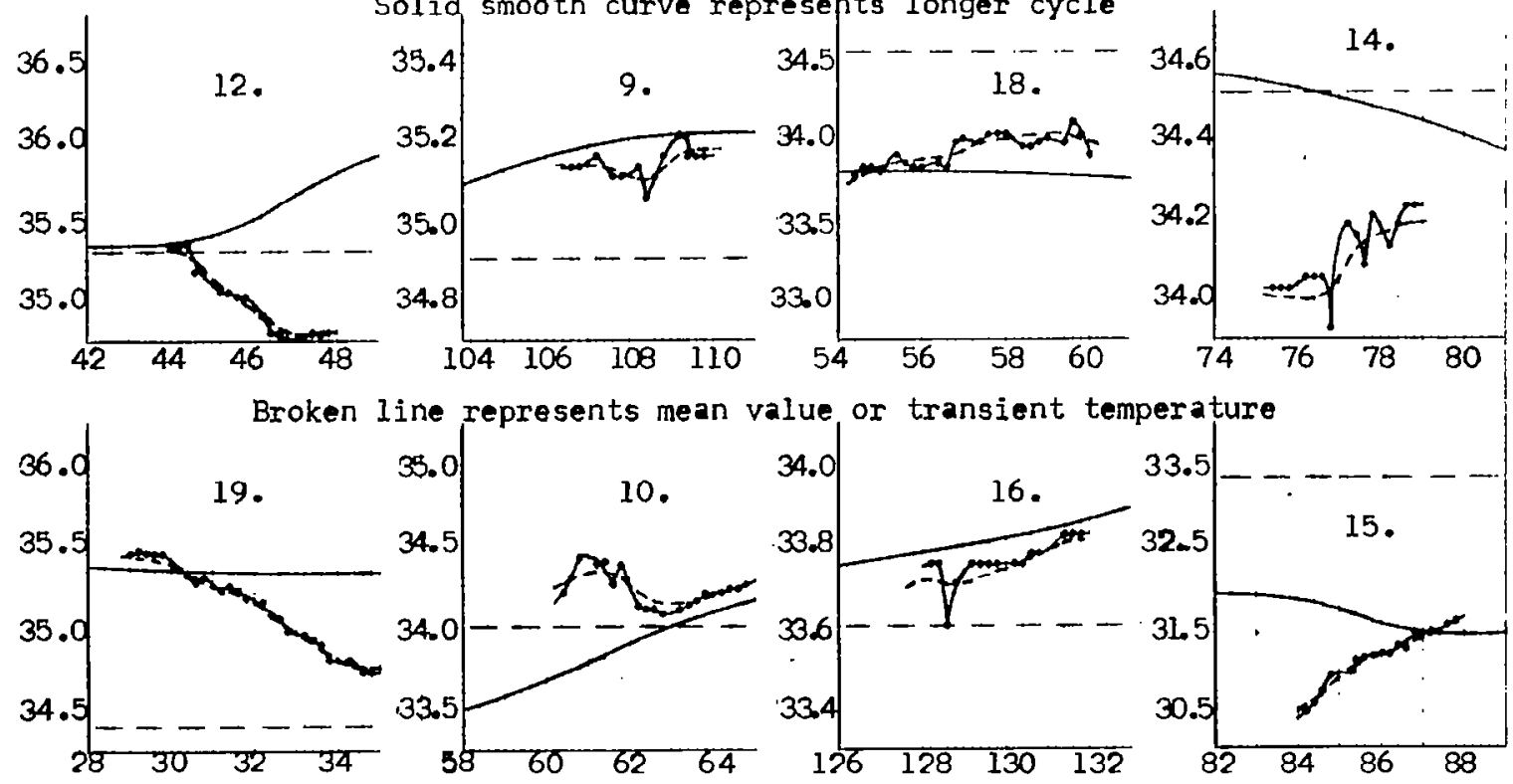

Chronological time scale at indicated hundreds of seconds 
Fig. 1i. High frequency skin temperature data continued
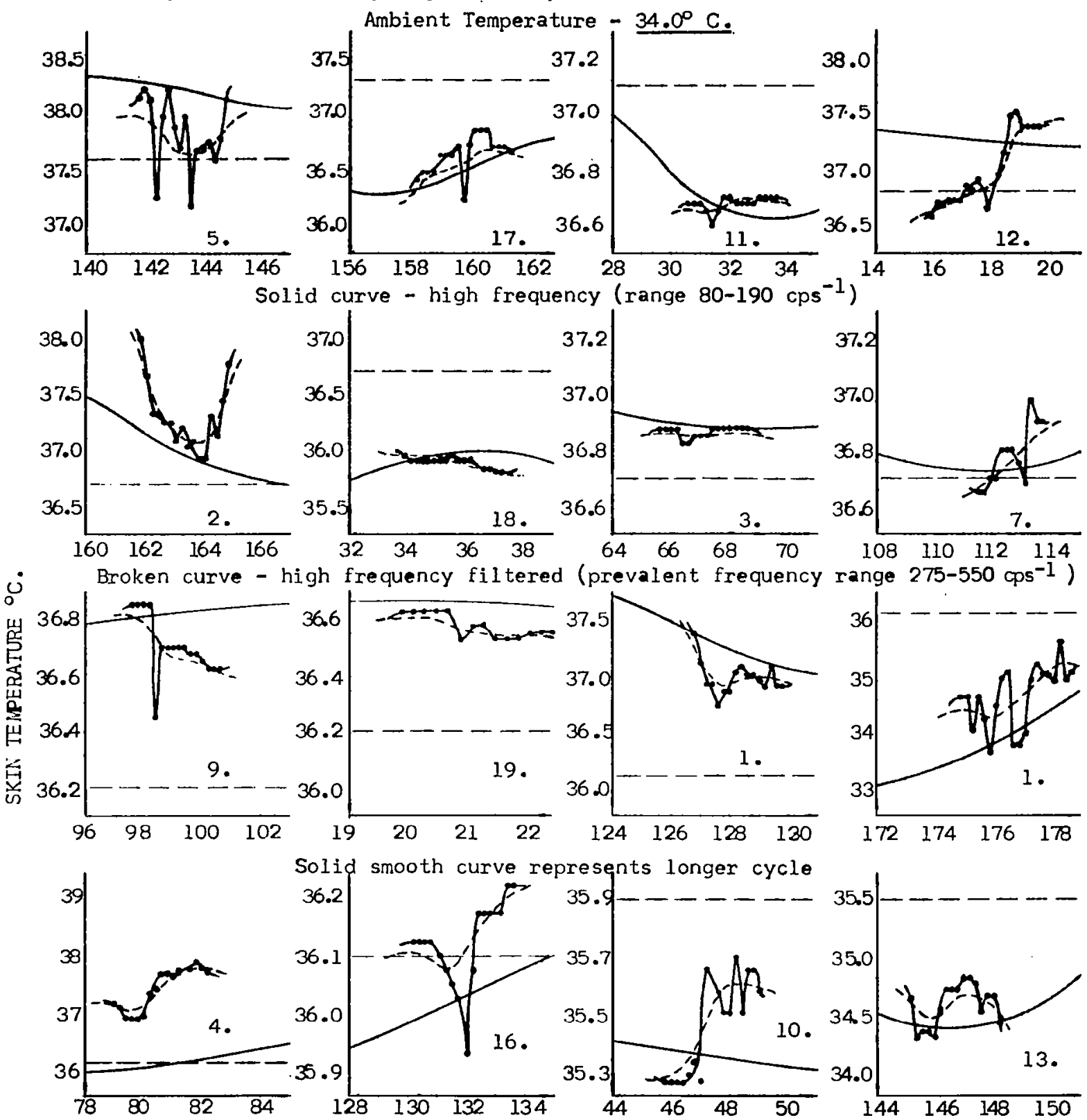

Solid smooth curve represents longer cycle
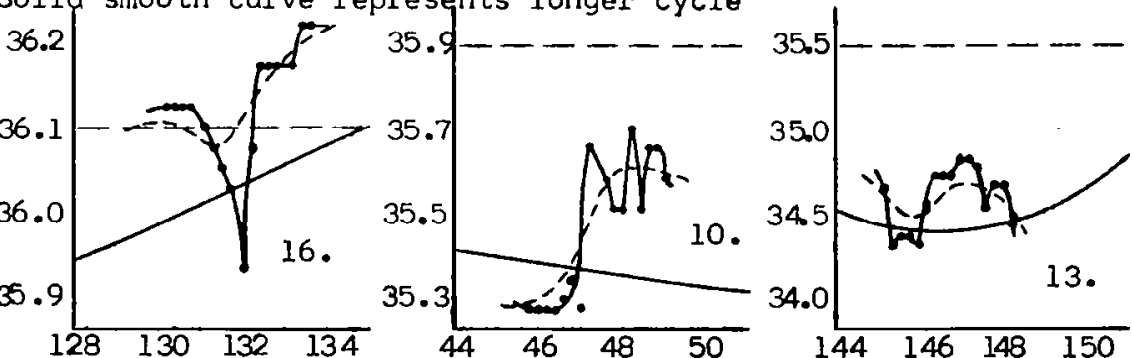

Broken line represents mean value or transient temperature

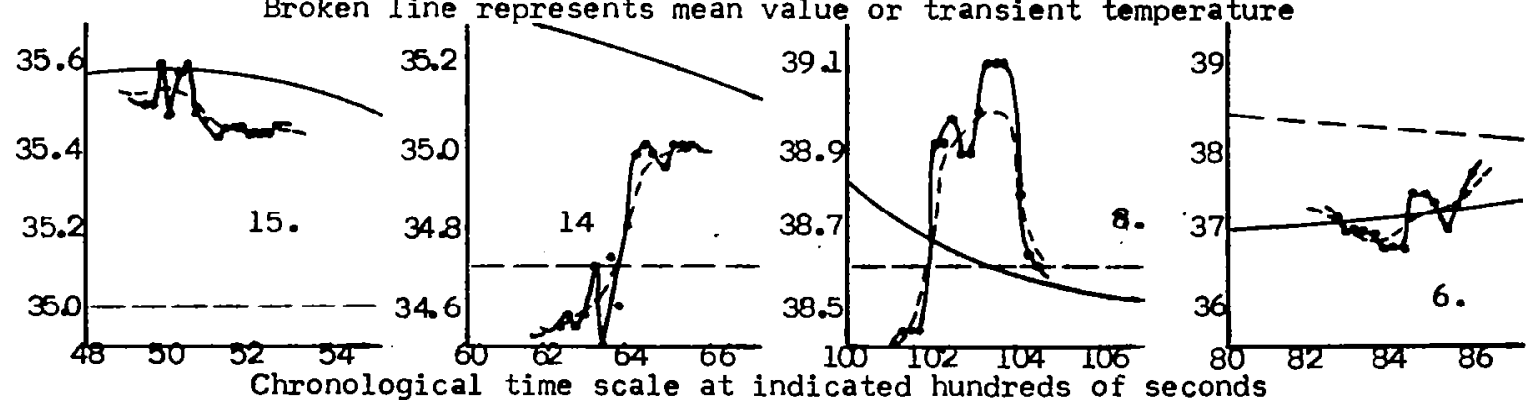




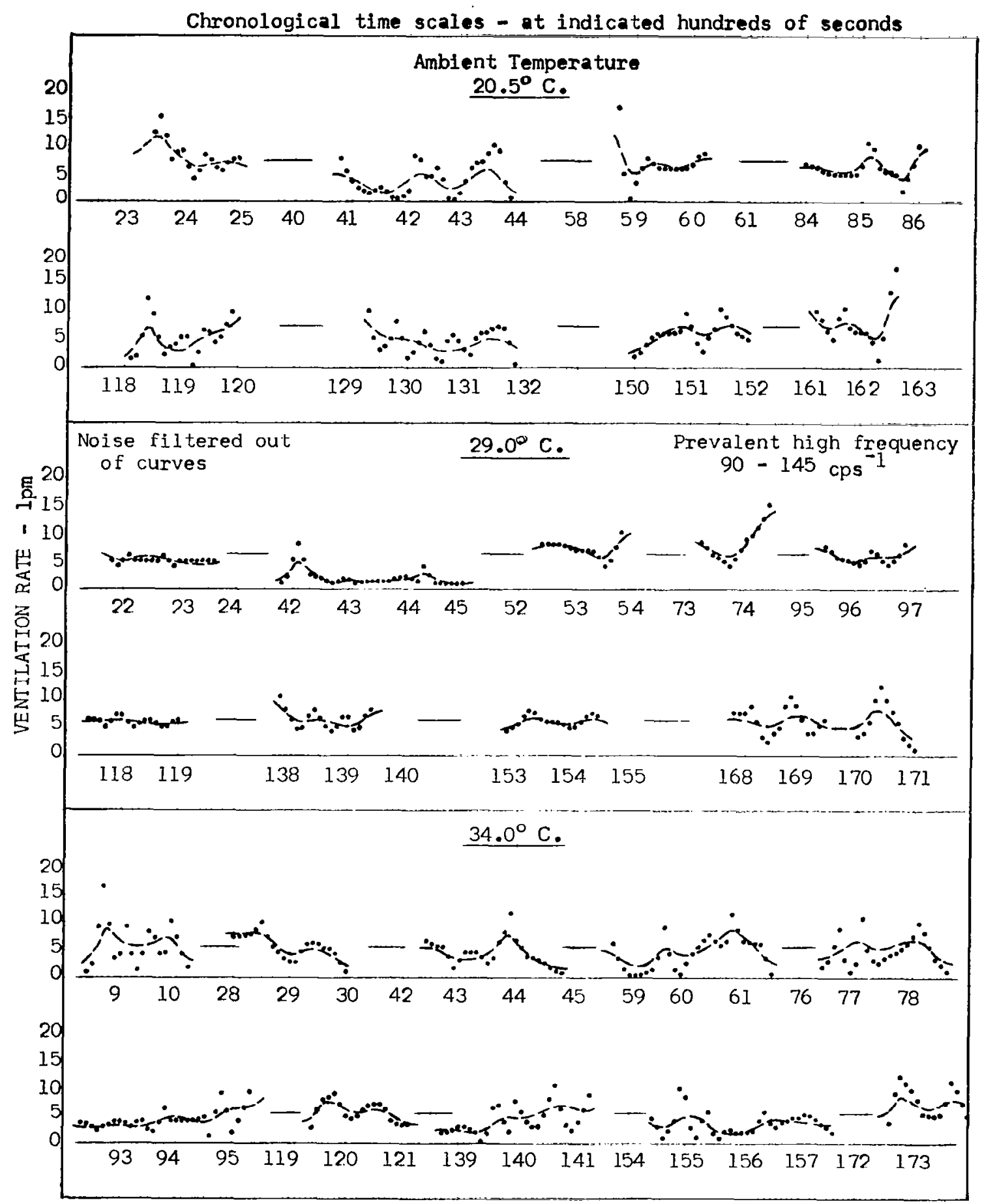

Fig. 1j. High frequency ventilation data for supine human subject previously described (Fast data) 


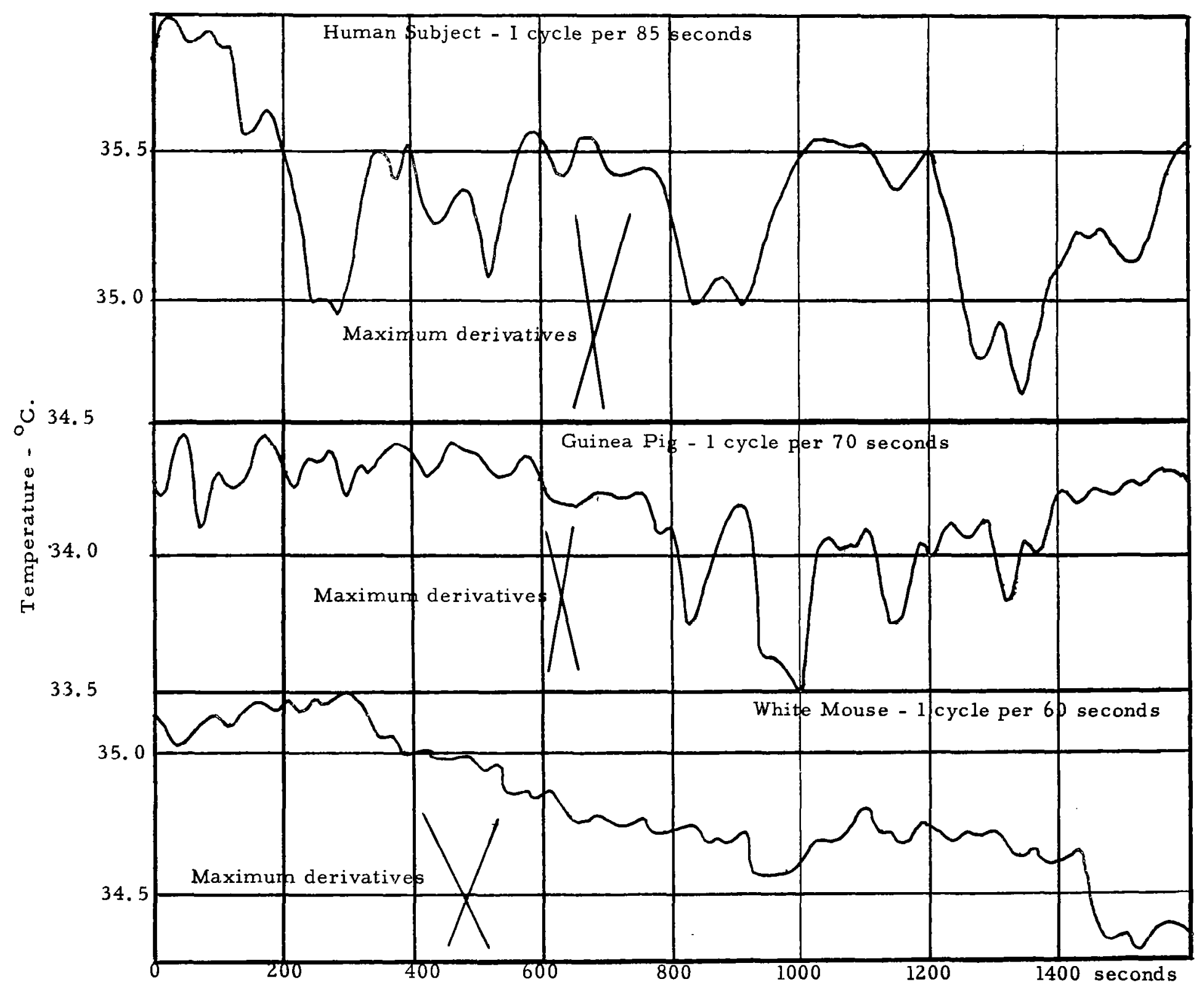

Figure 2. Course of skin temperature in time for some test animals and stations 


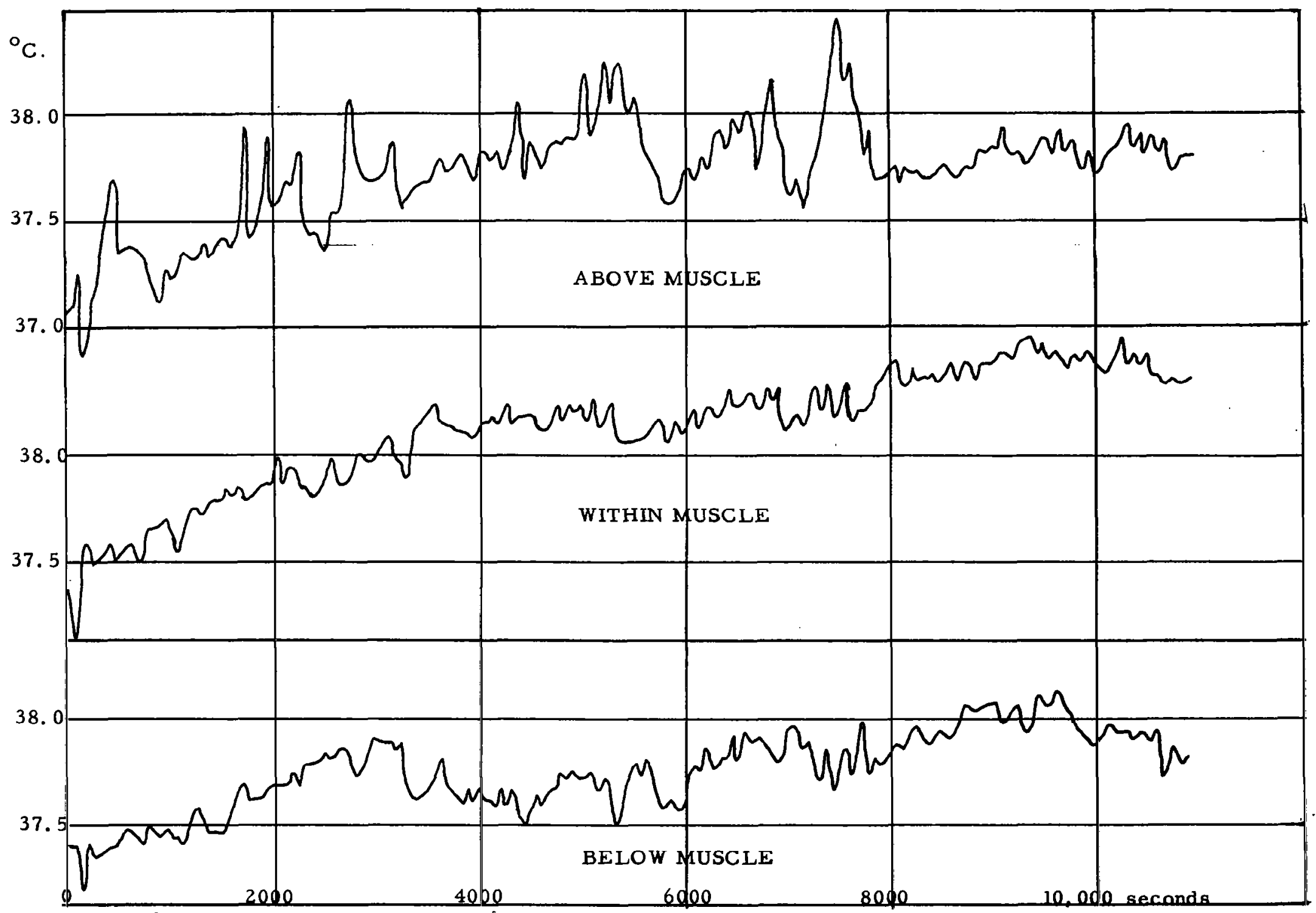

Figure 3a. Simultaneous temperature response above, within, and below the muscle sheath in quiescent test animal (guinea pig) (Experiment $19-2 / 17 / 64-3$ days after implant) 
${ }^{\circ} \mathrm{C}$

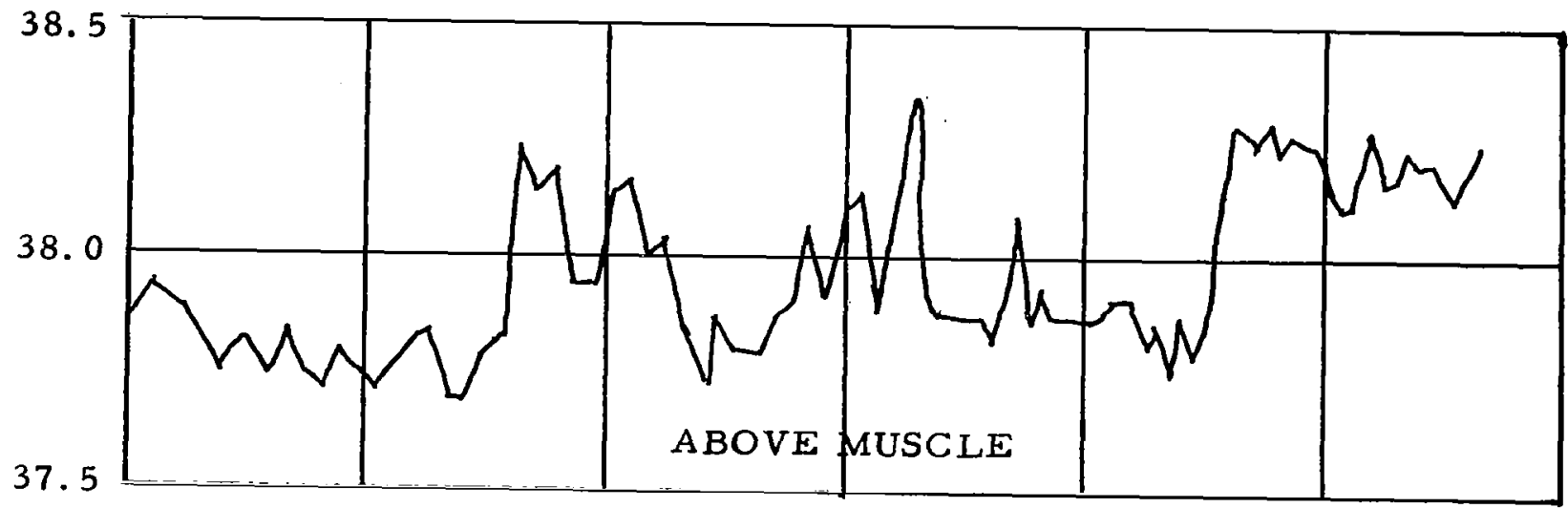

38.5

38.0

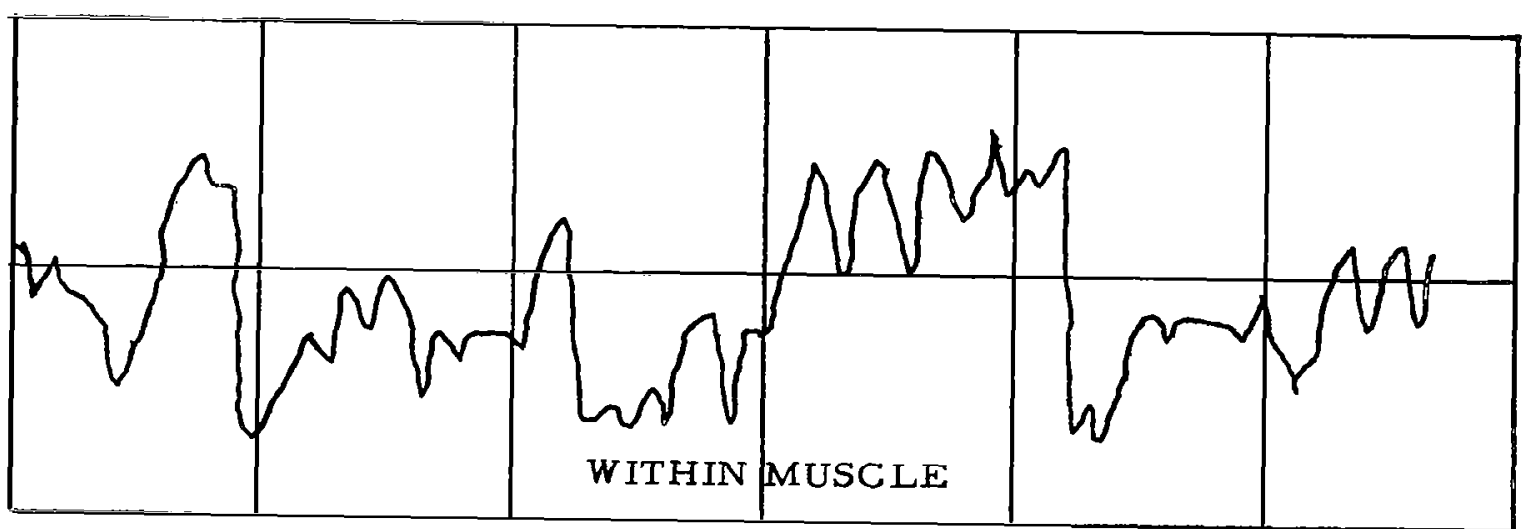

37.5

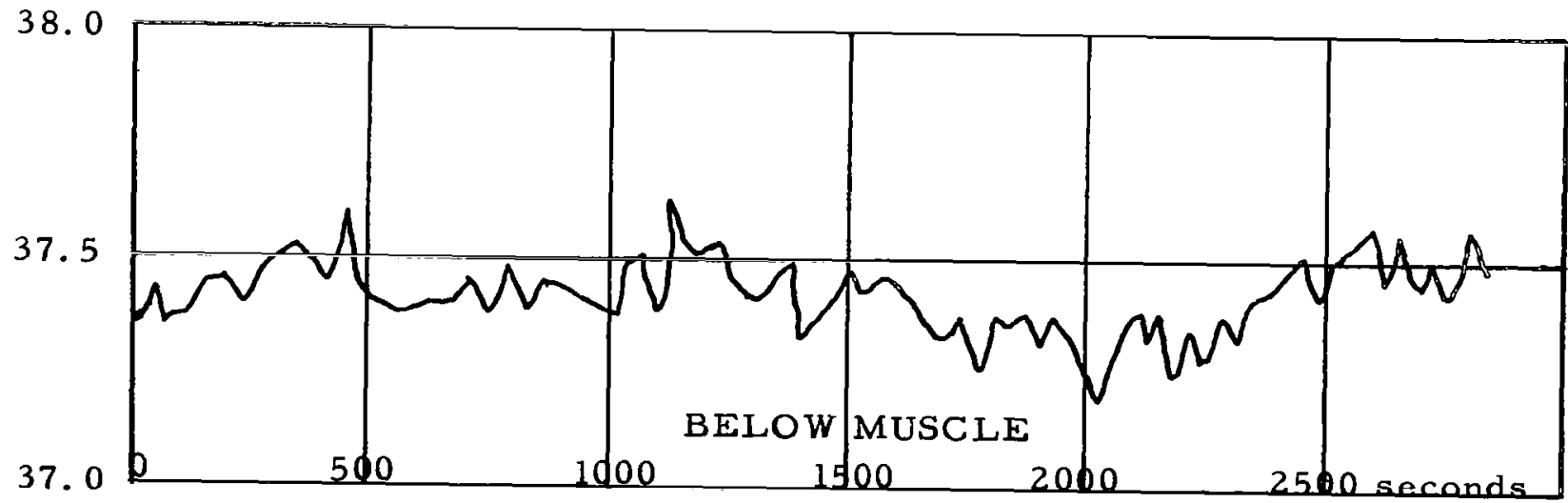

Figure 3b. Simultaneous temperature response above, within, and below the muscle sheath in quiescent test animal (guinea pig) (Experiment 20 - 2/18/64 - 4 days after implant - 

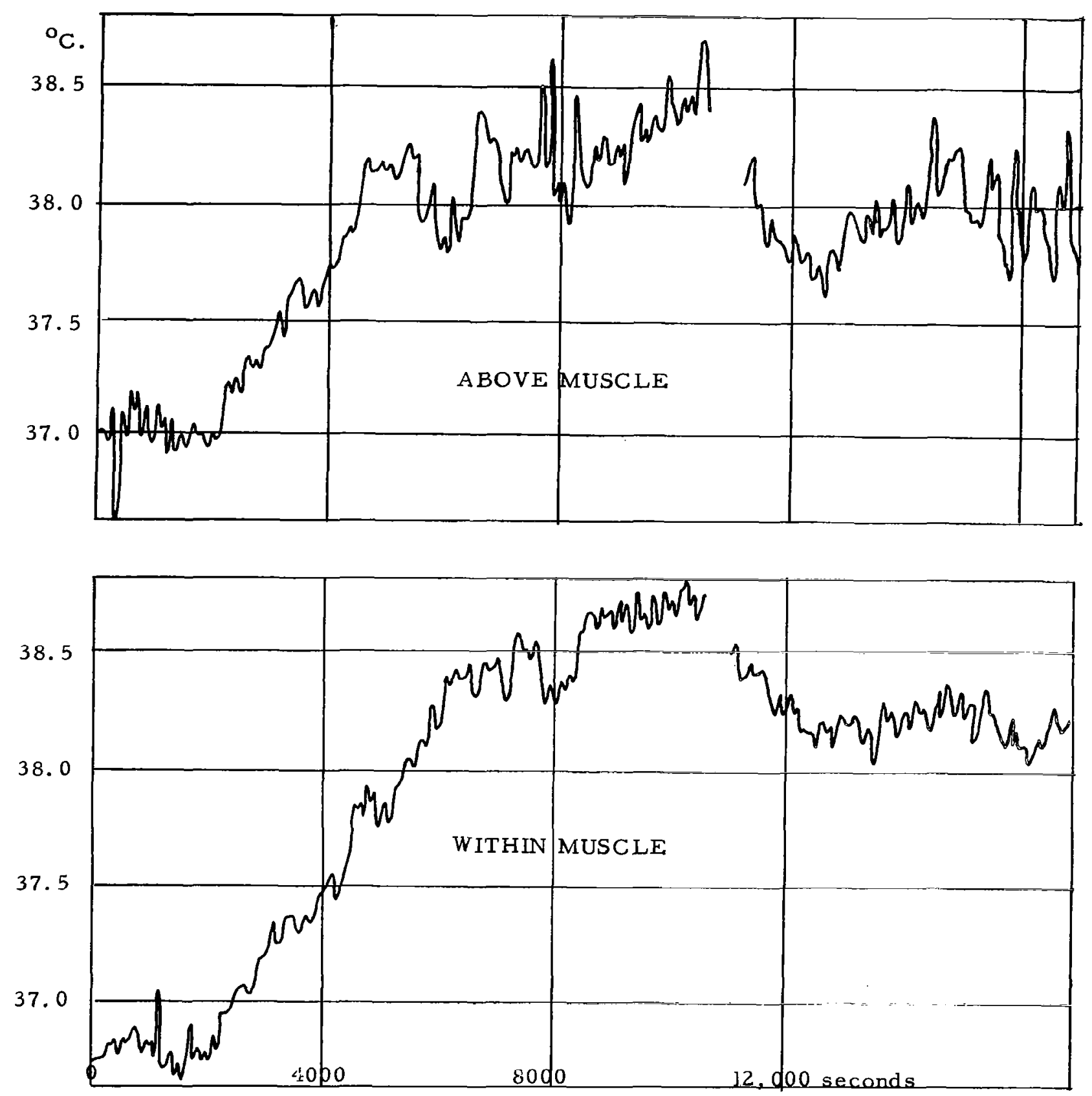

Figure 3c. Simultaneous temperature response above, within, and below the muscle sheath in quiescent test animal (guinea pig)

(Experiment 21 - 2/19/64 - 5 days after implant) 


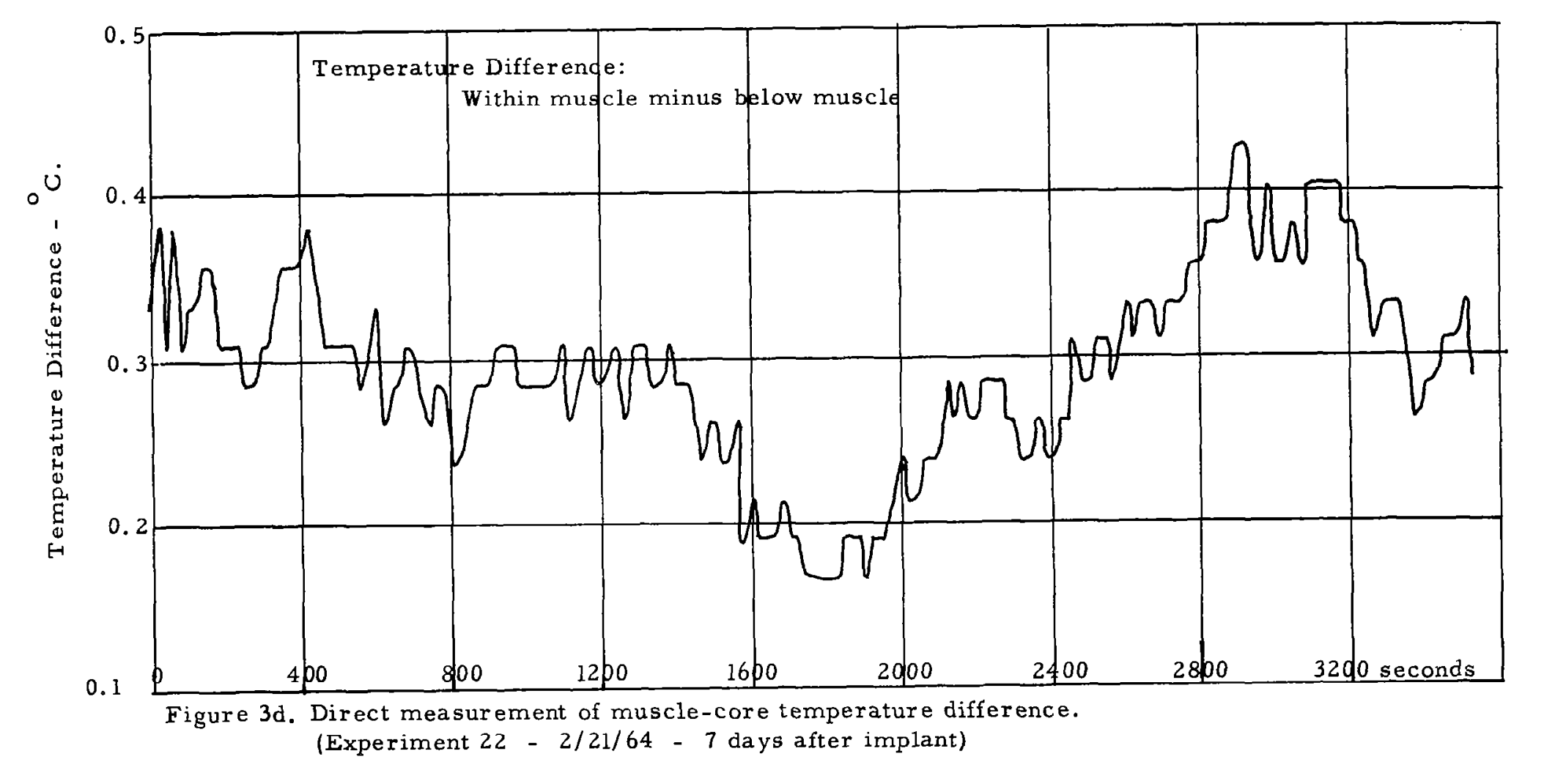



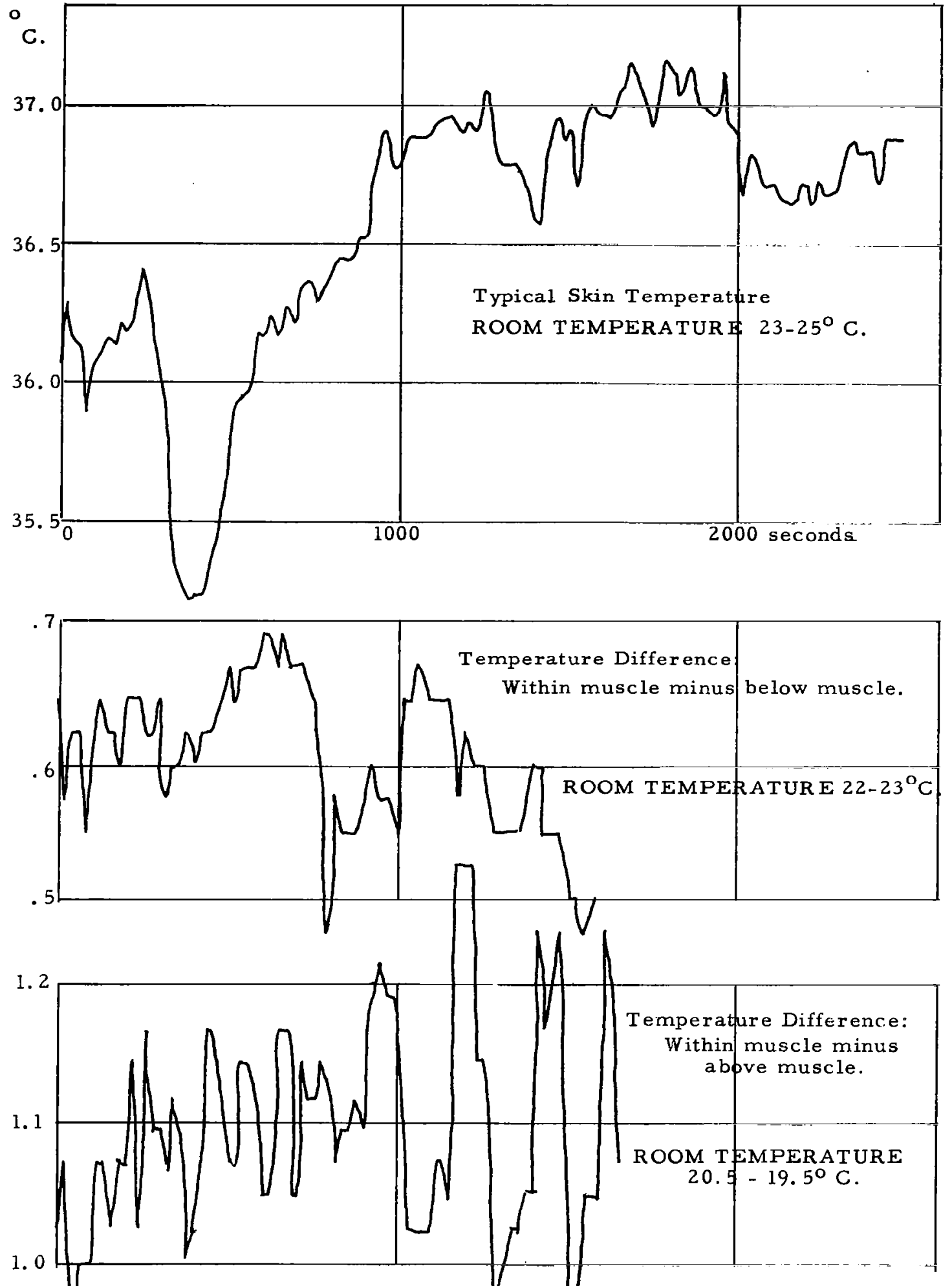

Figure 3e. Serial measurement of skin, muscle-core difference, and muscle-above muscle differelece in temperature.

(Experiment 23 - 2/22/64 - 8 days after implant) 


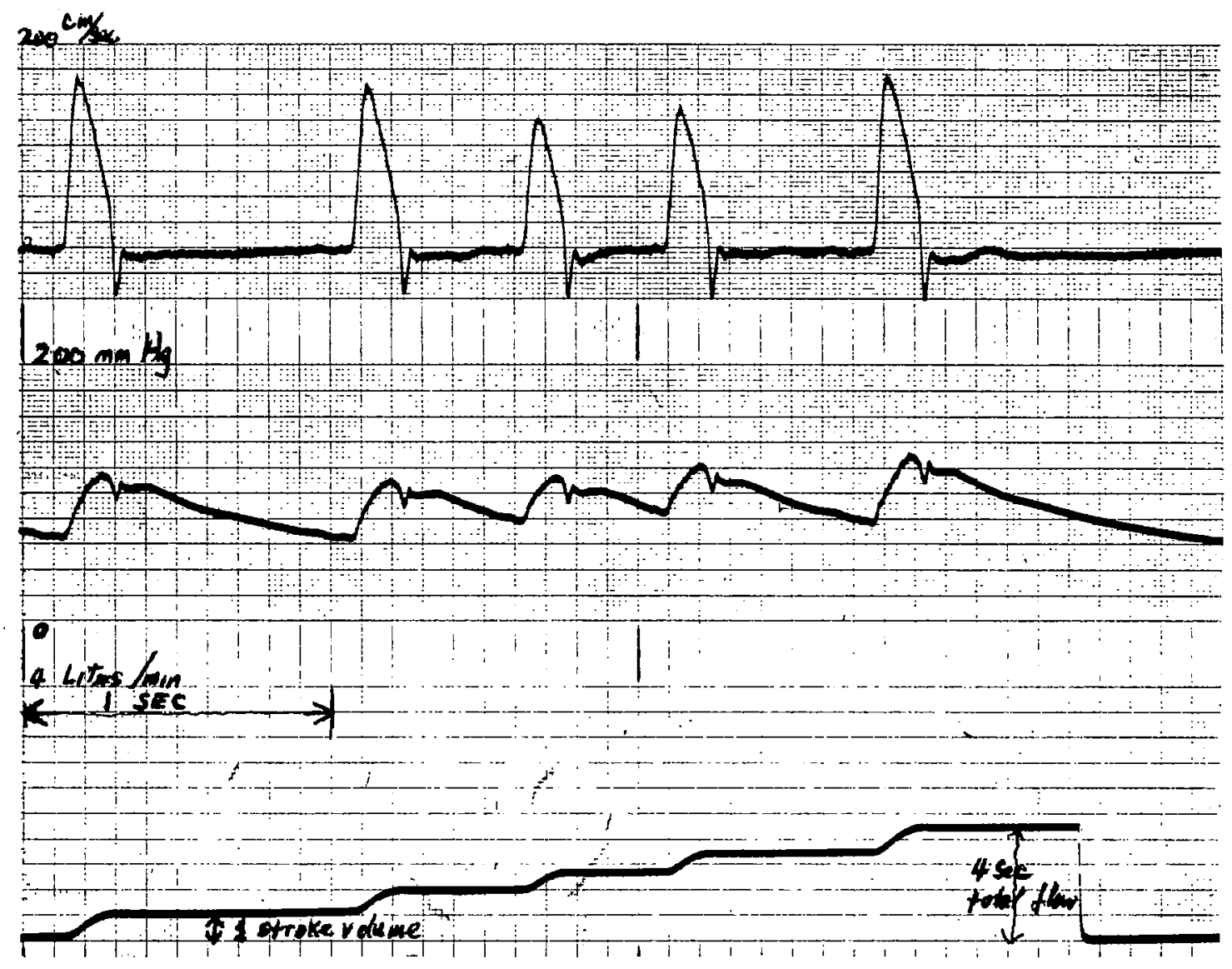

Figure 4. Mean velocity, pressure, integrated flow in ascending aorta

in unanesthetized quiescent dogs (Data source - Olmsted) 


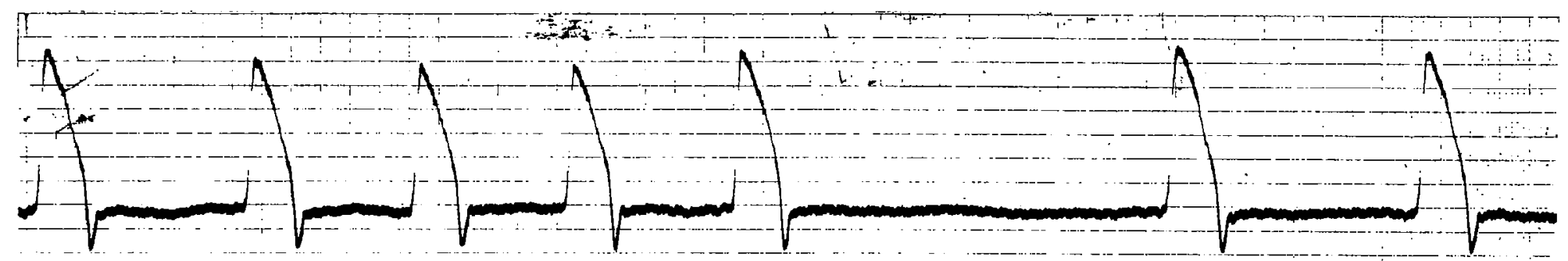

$883 H=3-1-63-L I E$
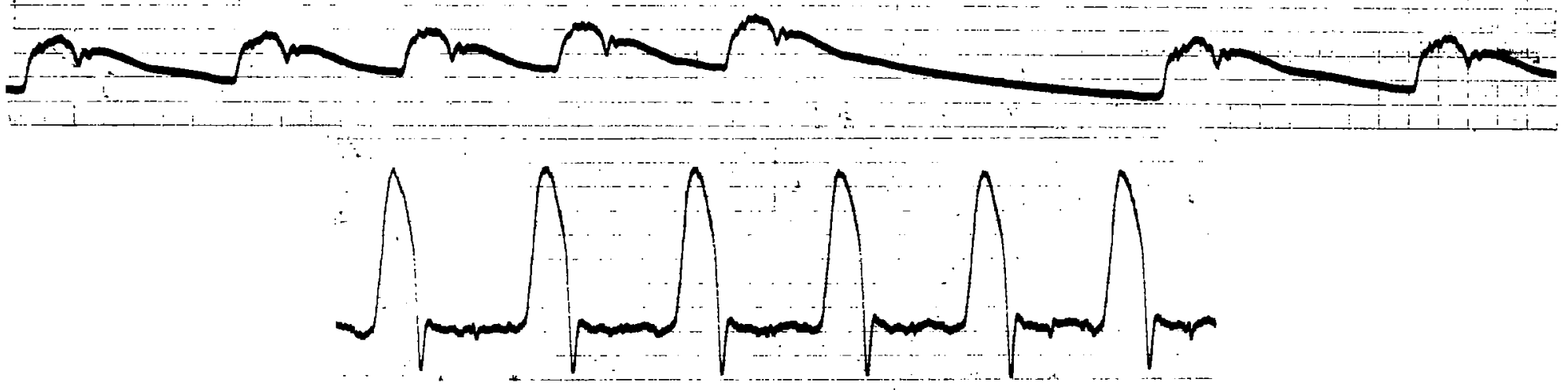

300

Fig. 4 (cont'd) 

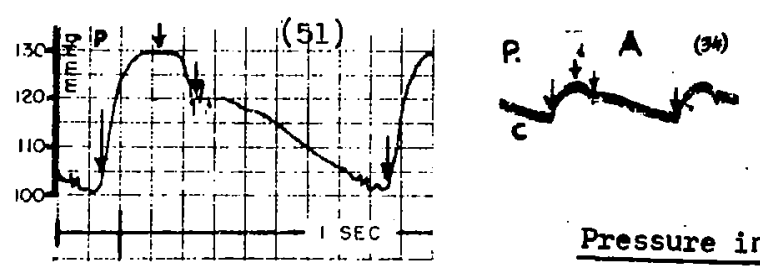

P. $\mathrm{CmiH}_{2}$ (4))

$\mathrm{cm} \mathrm{H} \mathrm{H}_{2} \mathrm{O}(50)$
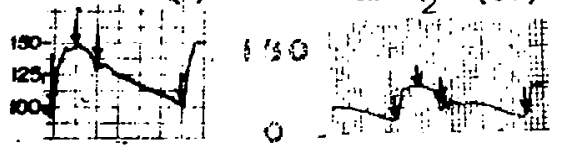

Pressure in Aortic Root

p.

$\therefore-1$ sec...

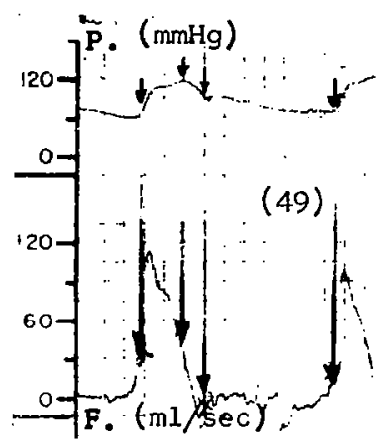

${ }_{00} F \cdot y^{(54)}$

${ }_{200}^{\text {P. }} t_{160}+t^{\prime}+1$
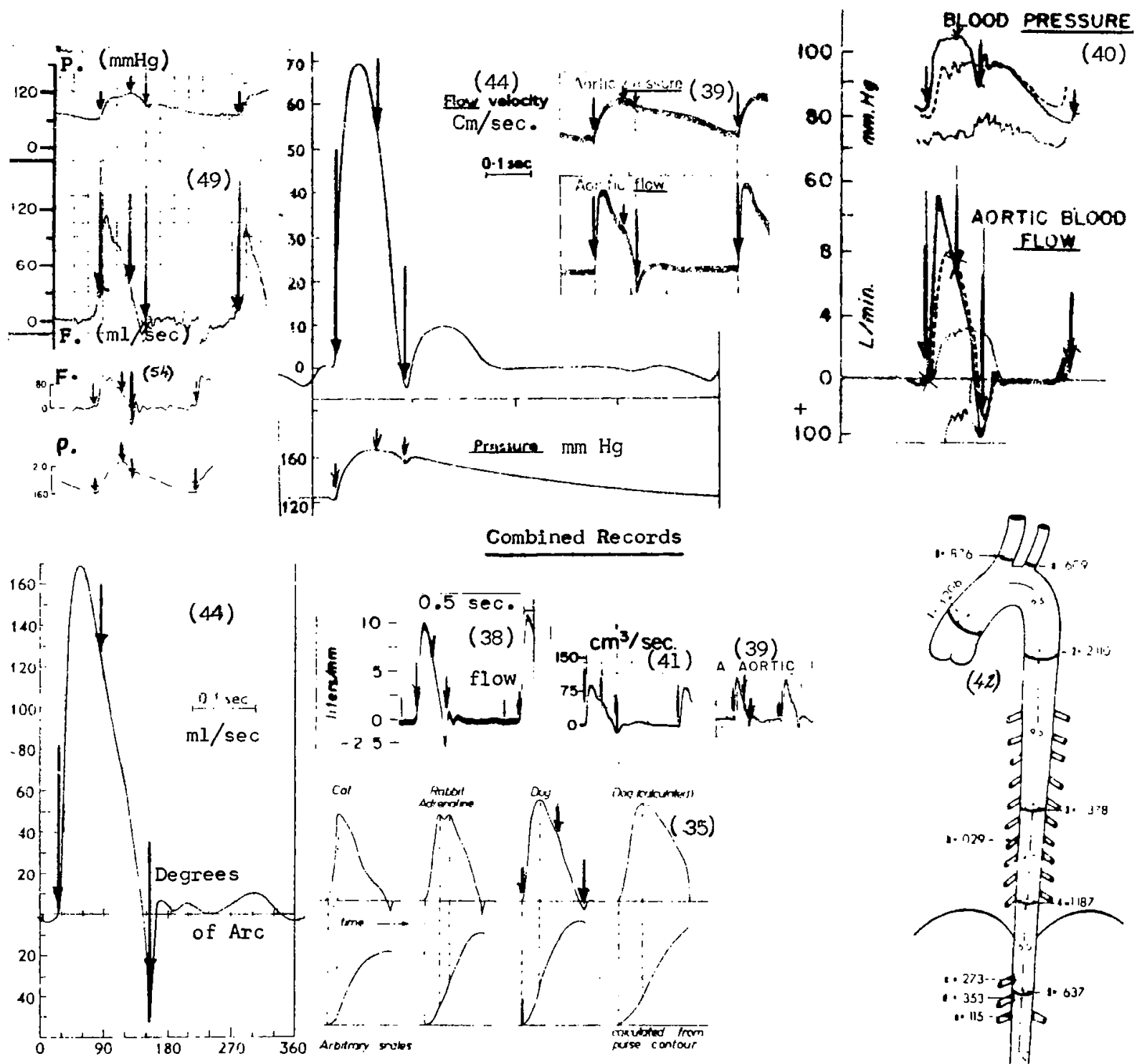

Flow in Aortic Root

NOTE: All records symchronized as far as possible to the salient points of the pressure wave in the ascending aorta of the start, the peak, the beginning of the notch, and the end.

Fig. 5a. PRESSURE AND FLOW EVENTS IN THE ARTERIAL SYSTEM

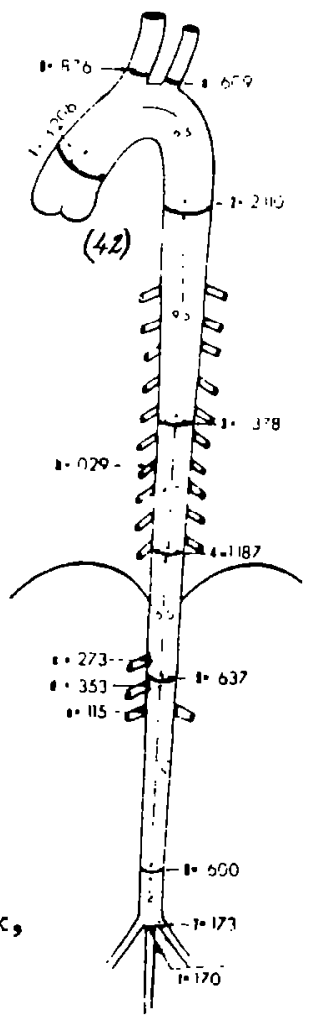

(Data from various investigators) - The Ascending Aorta 


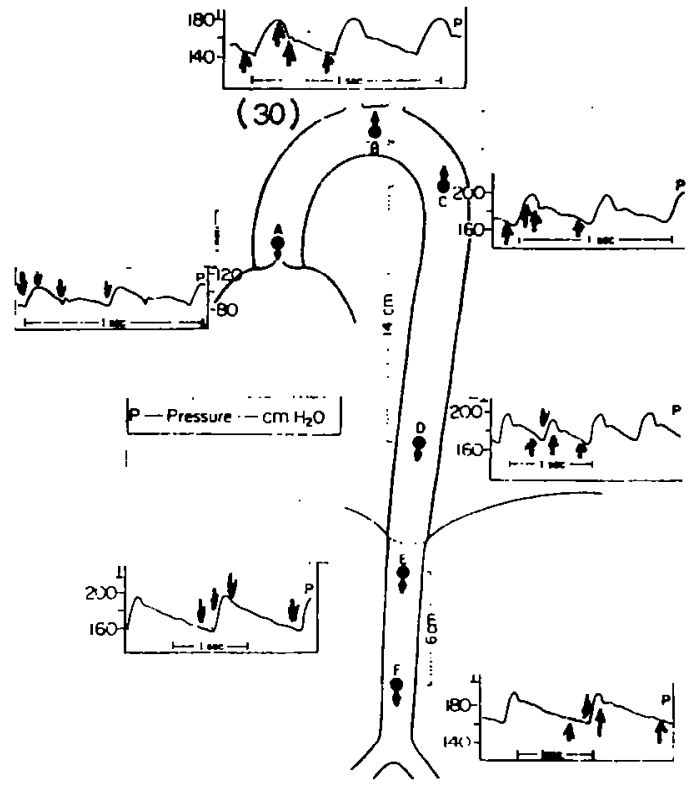

Pressure Sumnary Around the Aorta

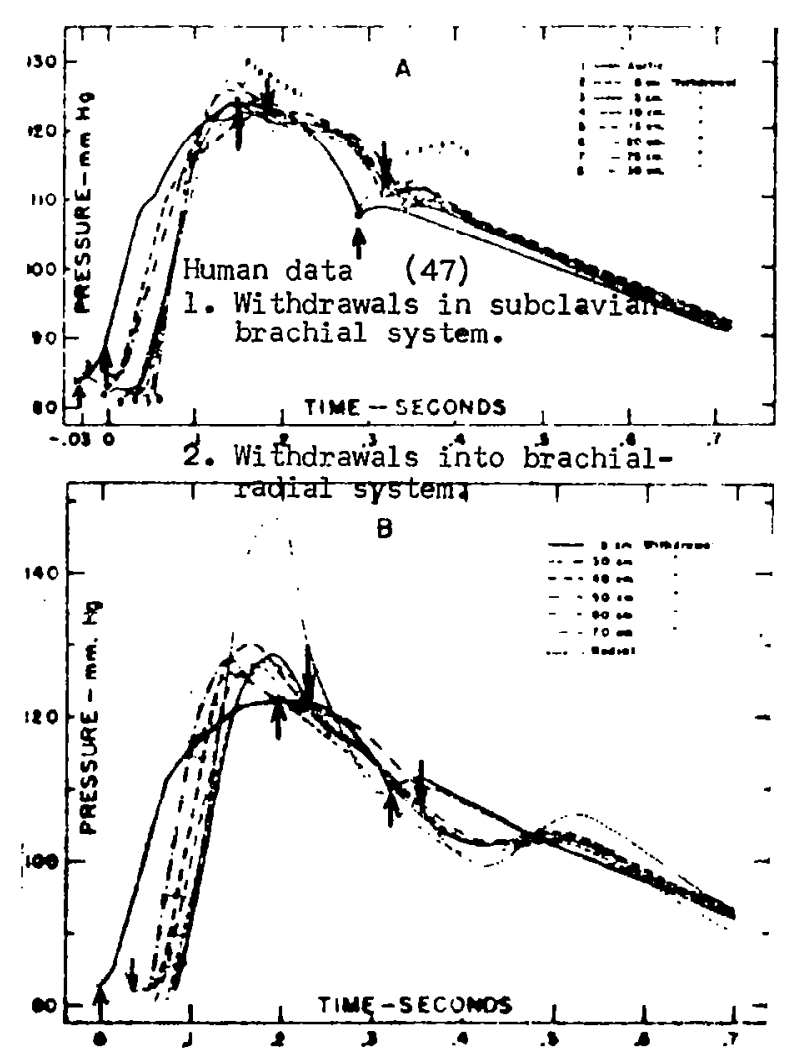

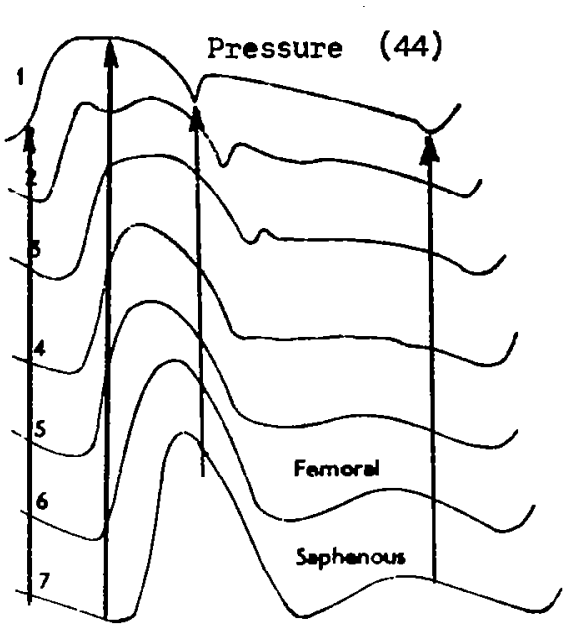
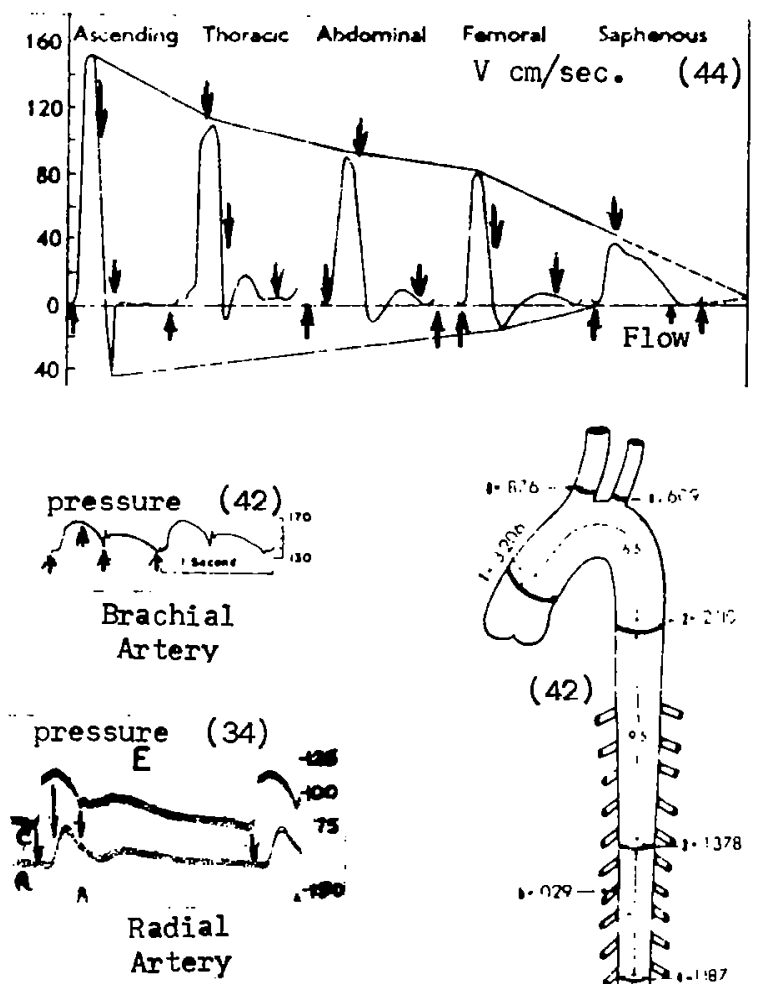

CAROTID ARTERY FlOW
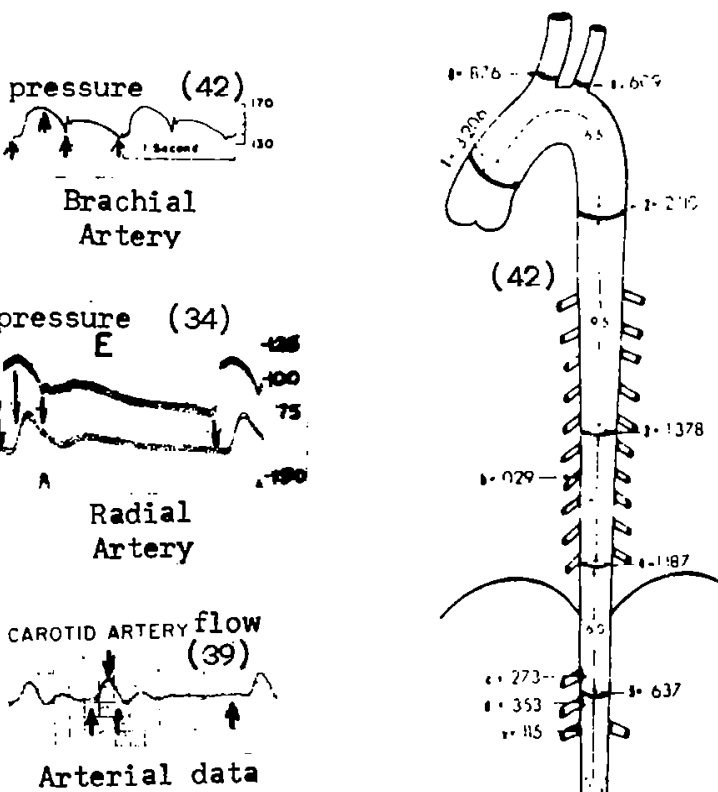

(42)
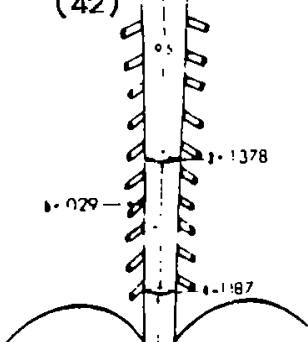

Fig. 5b. PRESSURE AND FLOW EVENTS IN THE ARTERIAL SYSTEM Aortic Arch 
$\int_{\text {Pressure }}(42)$ Pressure - Thoracic Aorta.
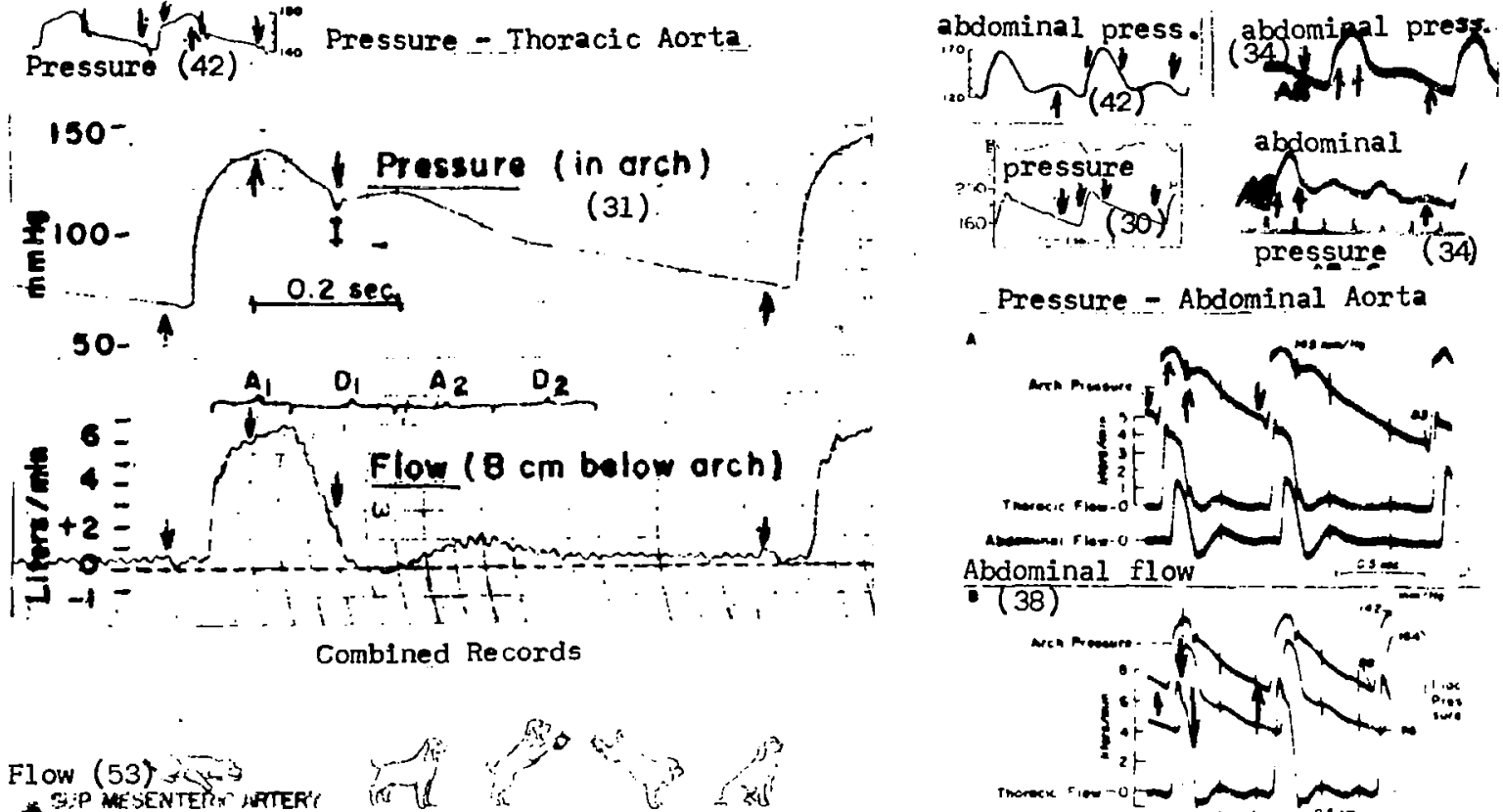

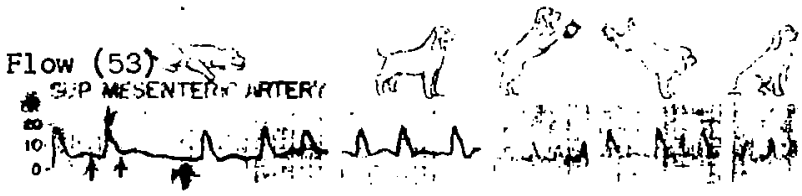

REHAi MRTEAY
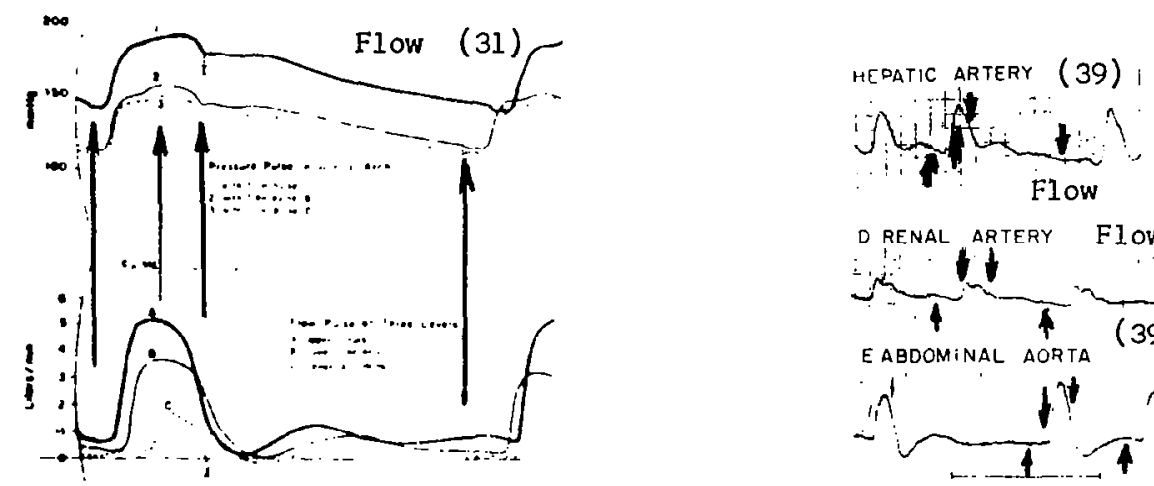

$$
\begin{aligned}
& \text { Thoracic flow : ase.:- }
\end{aligned}
$$

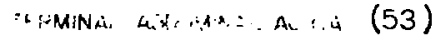

$$
\begin{aligned}
& \text { th }
\end{aligned}
$$

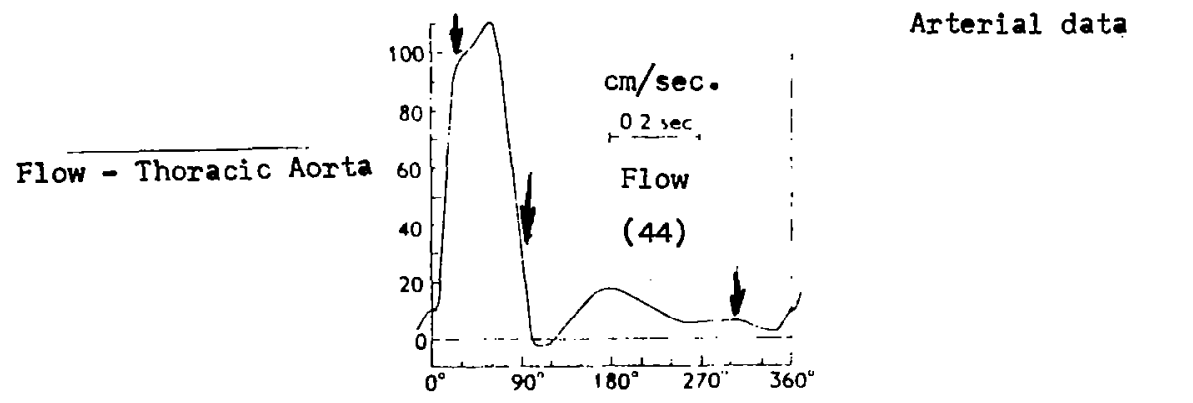

Fig. 5c. PRESSURE AND FLOW EVENTS IN THE ARTERIAL SYSTEM Thoracic and Abdominal Aorta

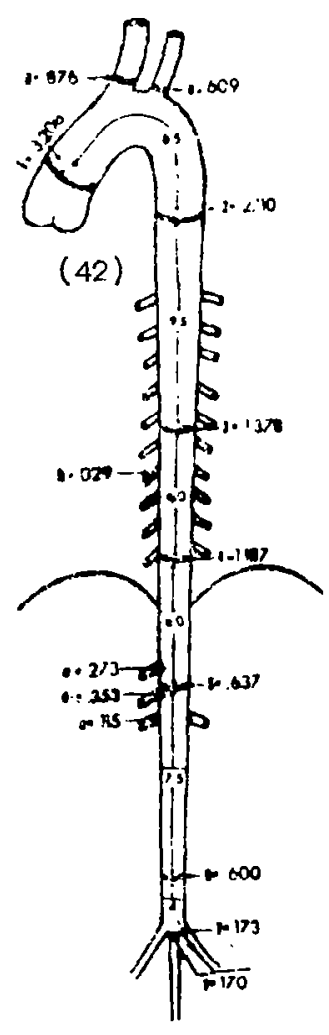



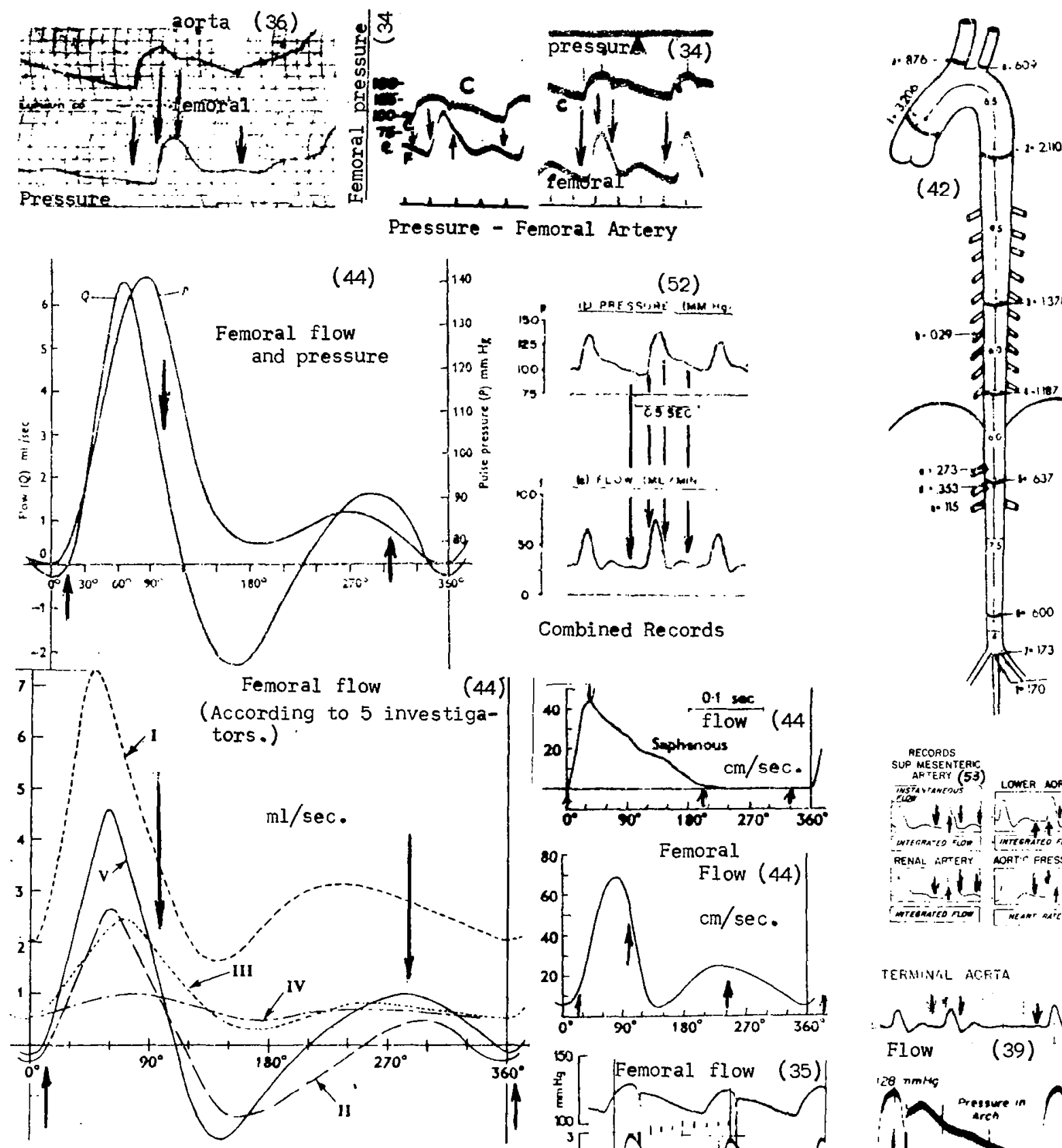

Combined Records

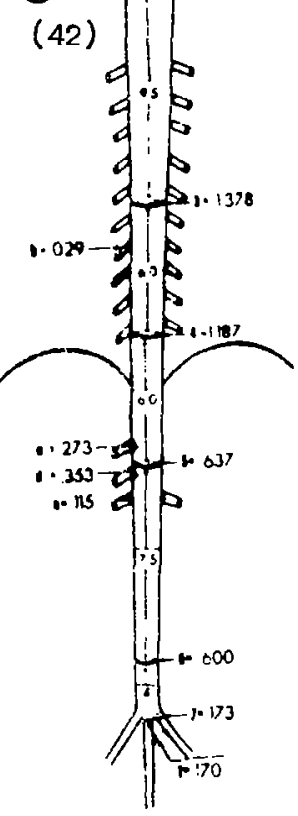

AECORDS

SUP MESENTERTC

AFTERT (53) LOWEA MORTA

U L t

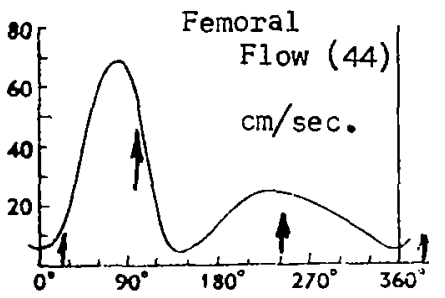

RENAL APTERY AOAT' FRESSURE

at

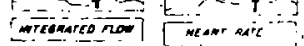

TEFMINIL ACFTA
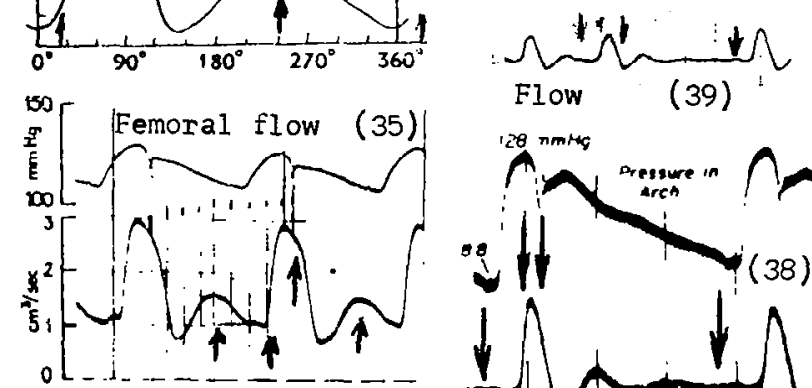

Flow-Femoral Axtery

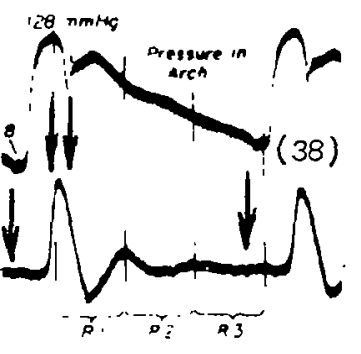

Fiow in Terminal Aorto

Terminal Aorta

Fig. 5d. PRESSURE AND FLOW EVENTS IN THE ARTERIAL SYSTEM Terminal Aorta 

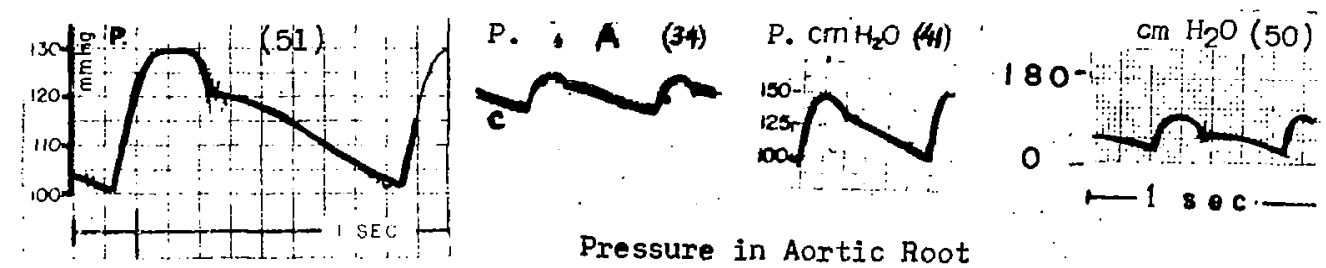

Pressure in Aortic Root
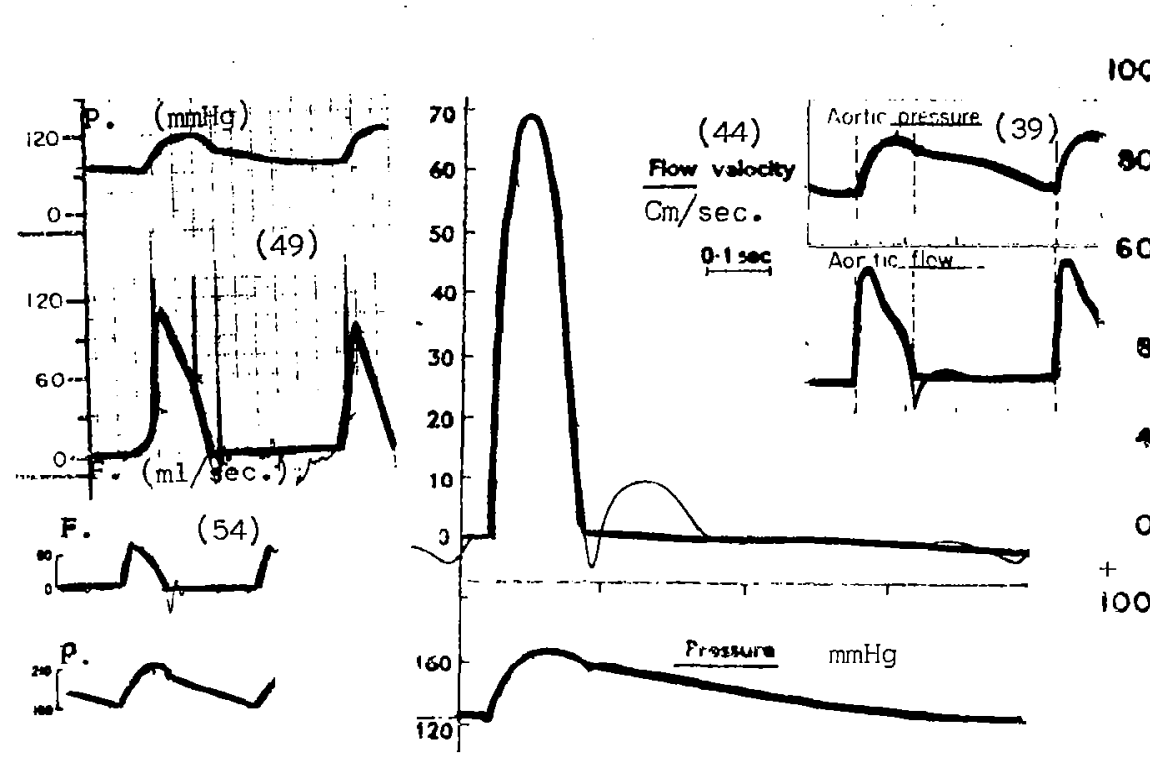

Combined Records
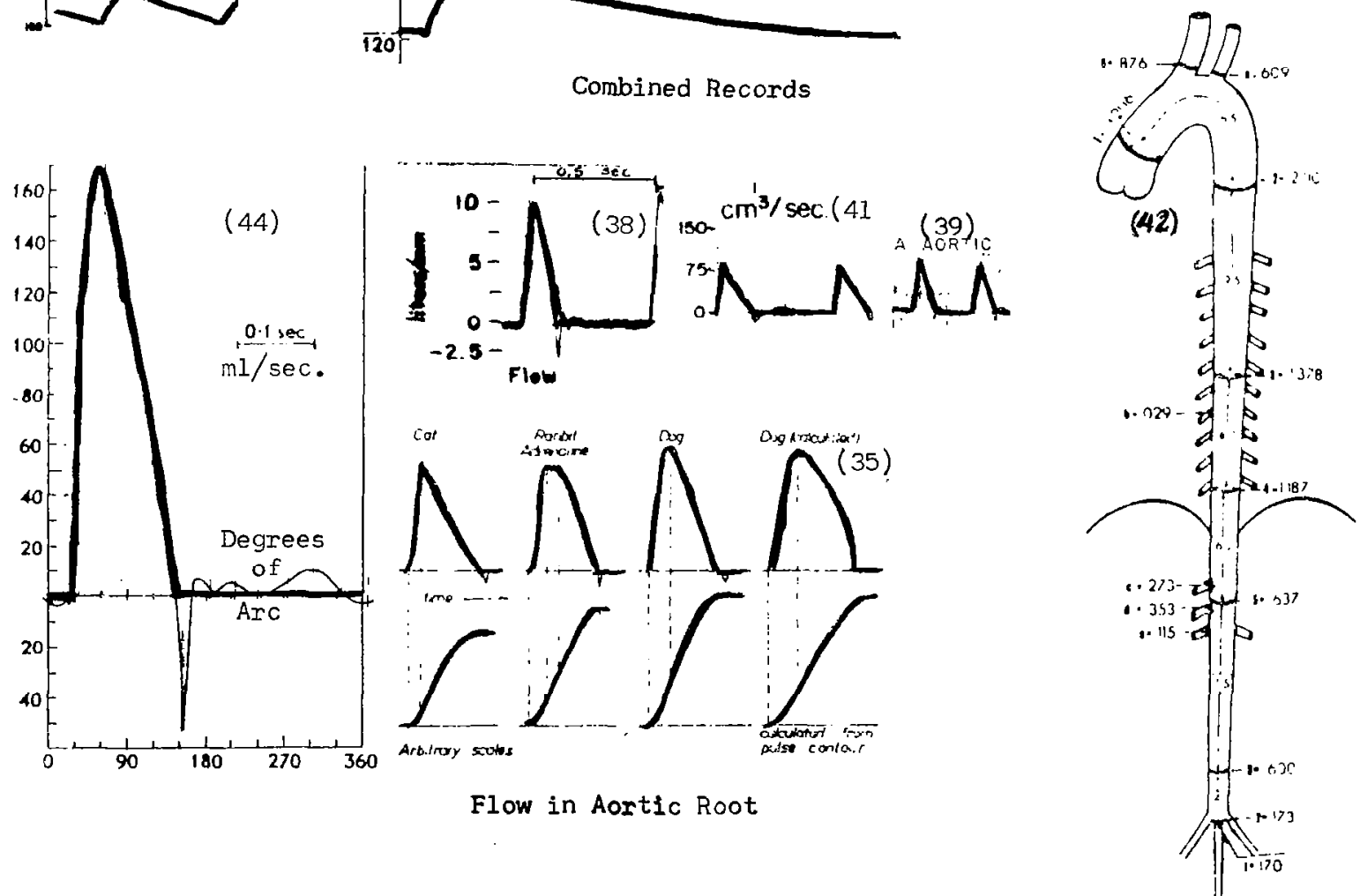

Fig. 5e. PRESSURE AND FLOW EVENTS IN THE ARTERIAL SYSTEM FILTERED FREE OF DICHROTIC NOTCH AND DICHROT IC WAVE OSCILLATIONS 


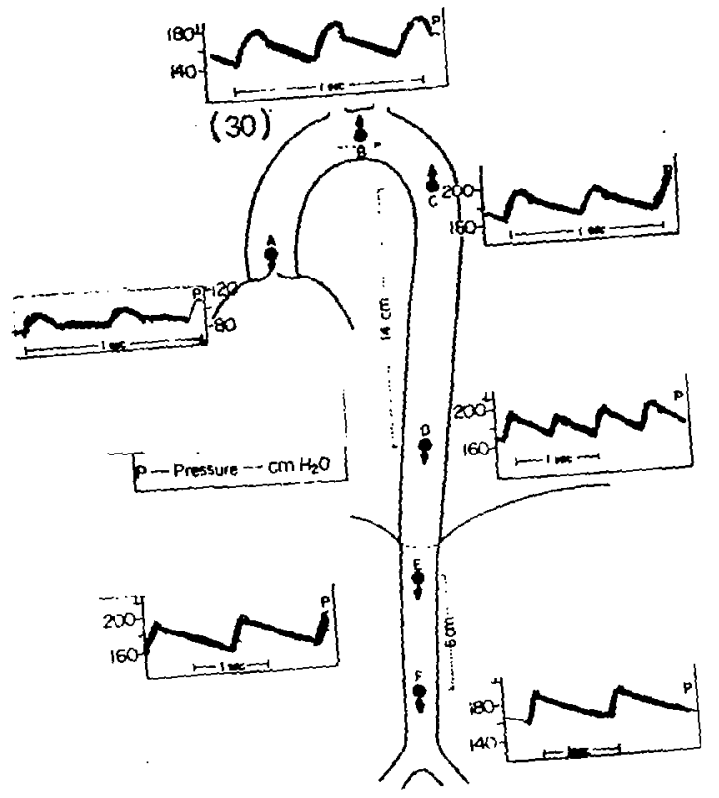

pressure Summary Around the Aorta
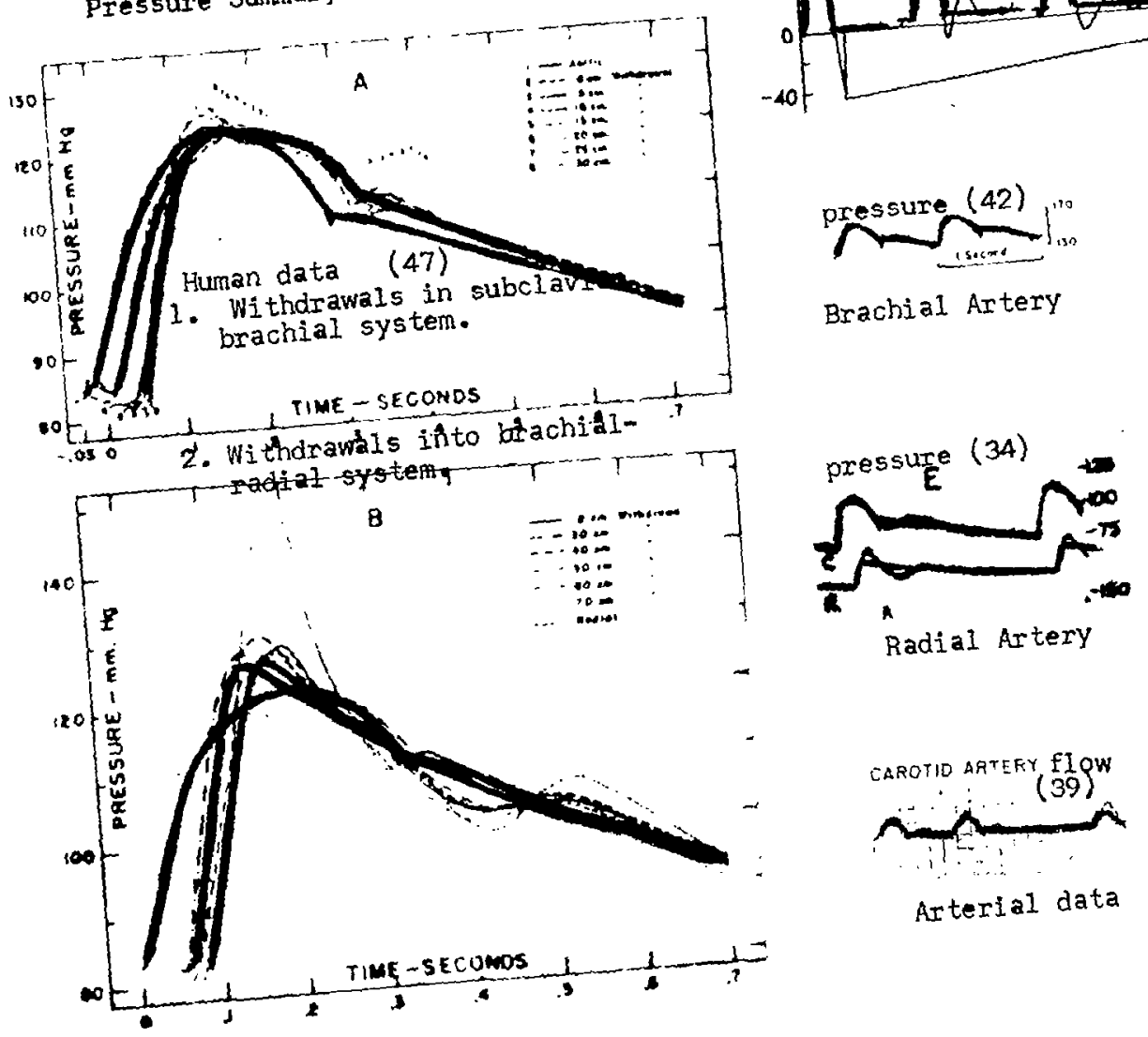

Brachial Artery
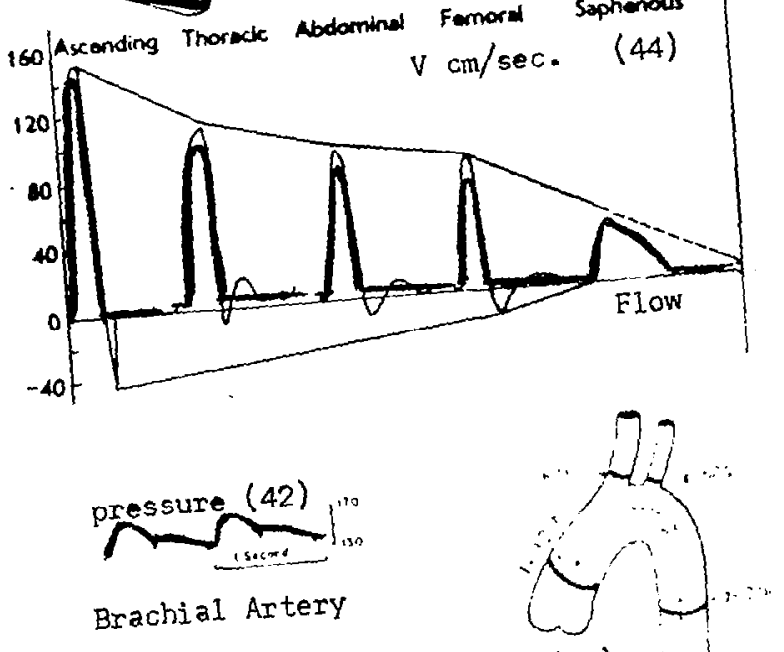

(42)

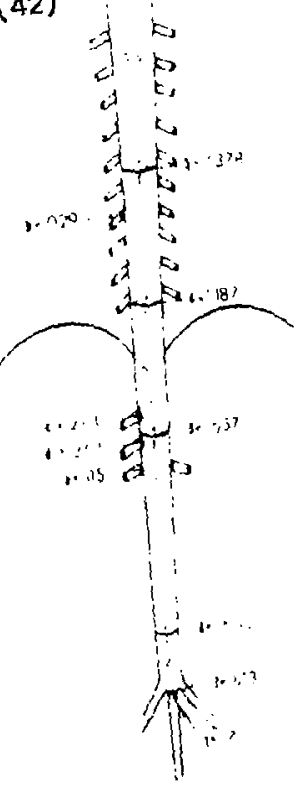

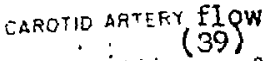

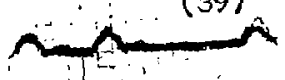

Arterial data

(44) 
$\overbrace{\text { Pressure (42) }}^{100}$ Pressure - Thoracic Aorta
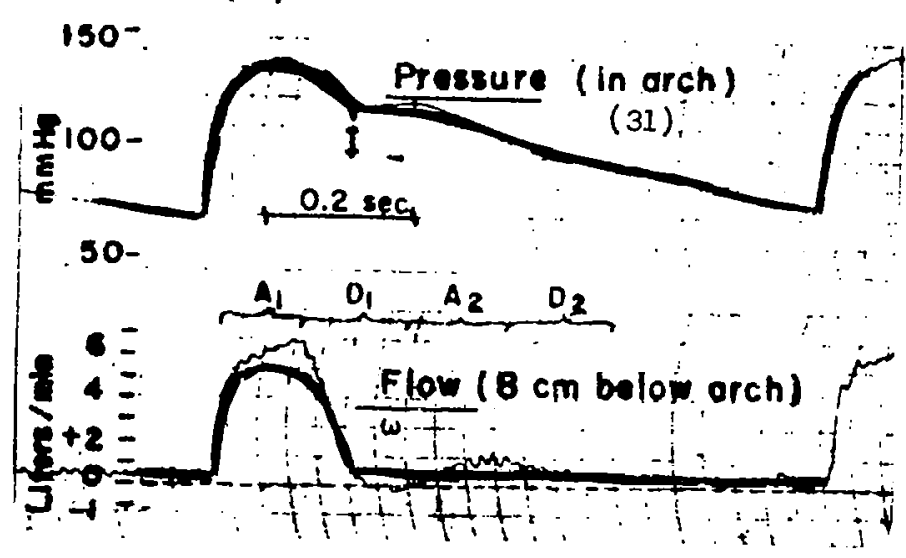

Combined Records
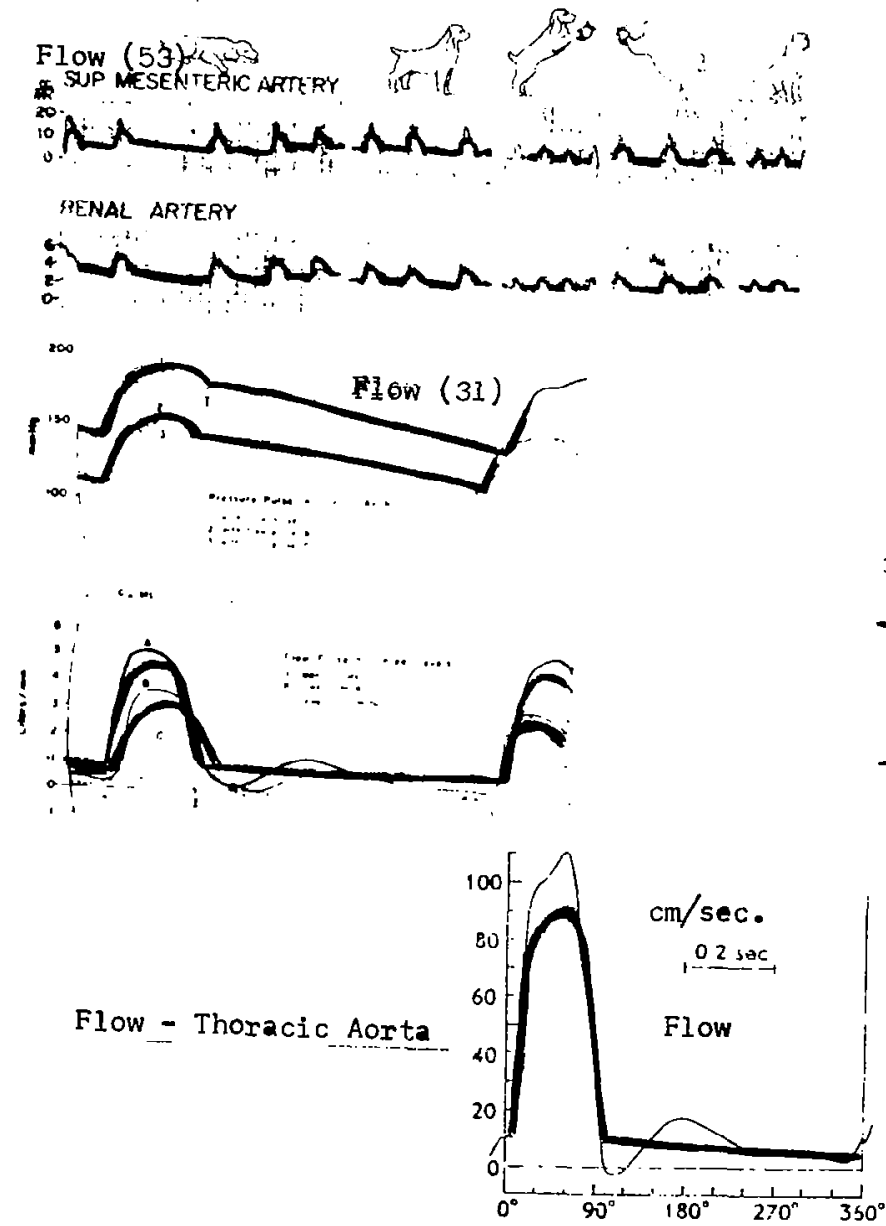

Fig. 5g. PRESSURE AND FLOW EVENTS IN THE ARTERIAL SYSTEM Thoracic and Abdominal Aorta
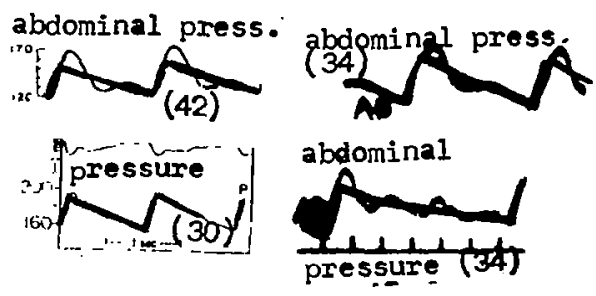

Pressure - Abdominal Aorta

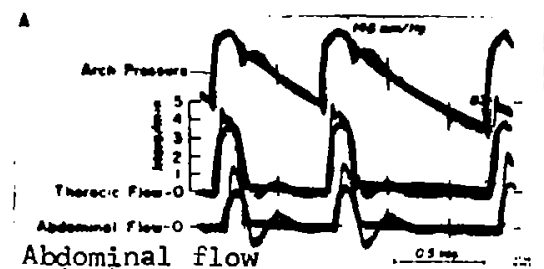

Abdominal flow

(38)

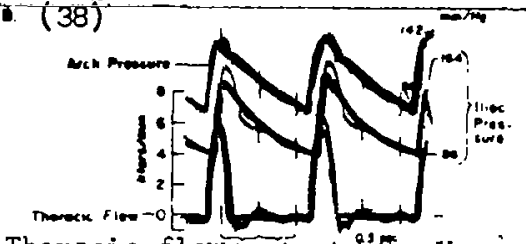

Thoracic flow: is

TEFMINAL ABCKMINAL AORTA

(53)
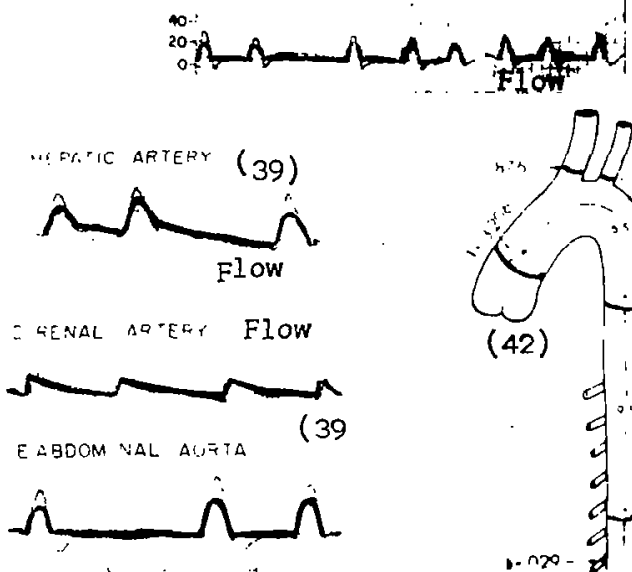

Arterial data
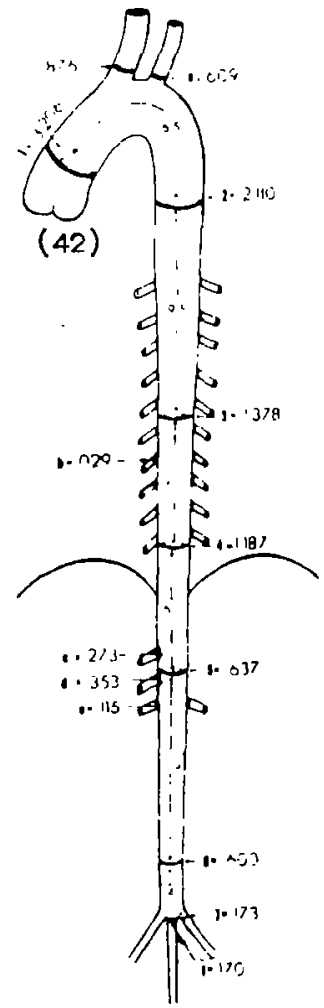


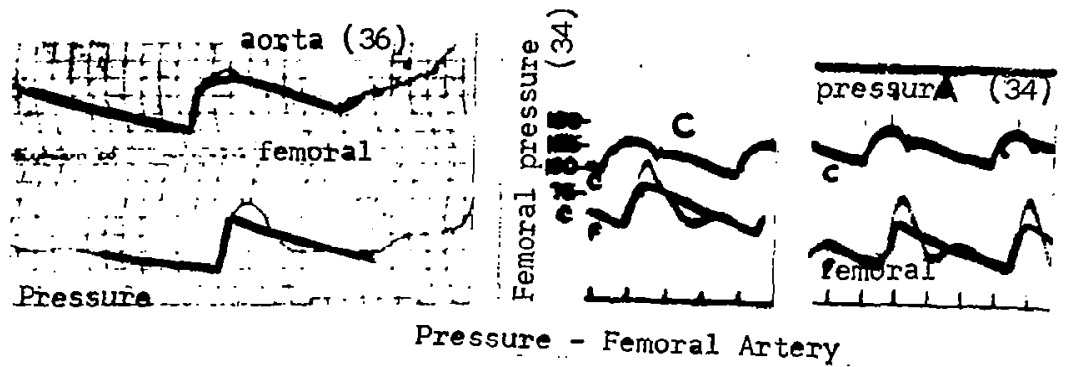

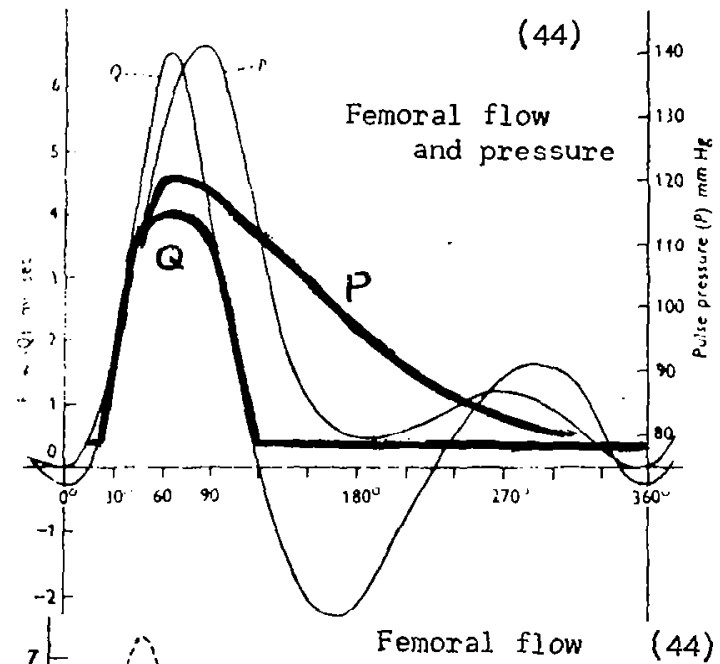
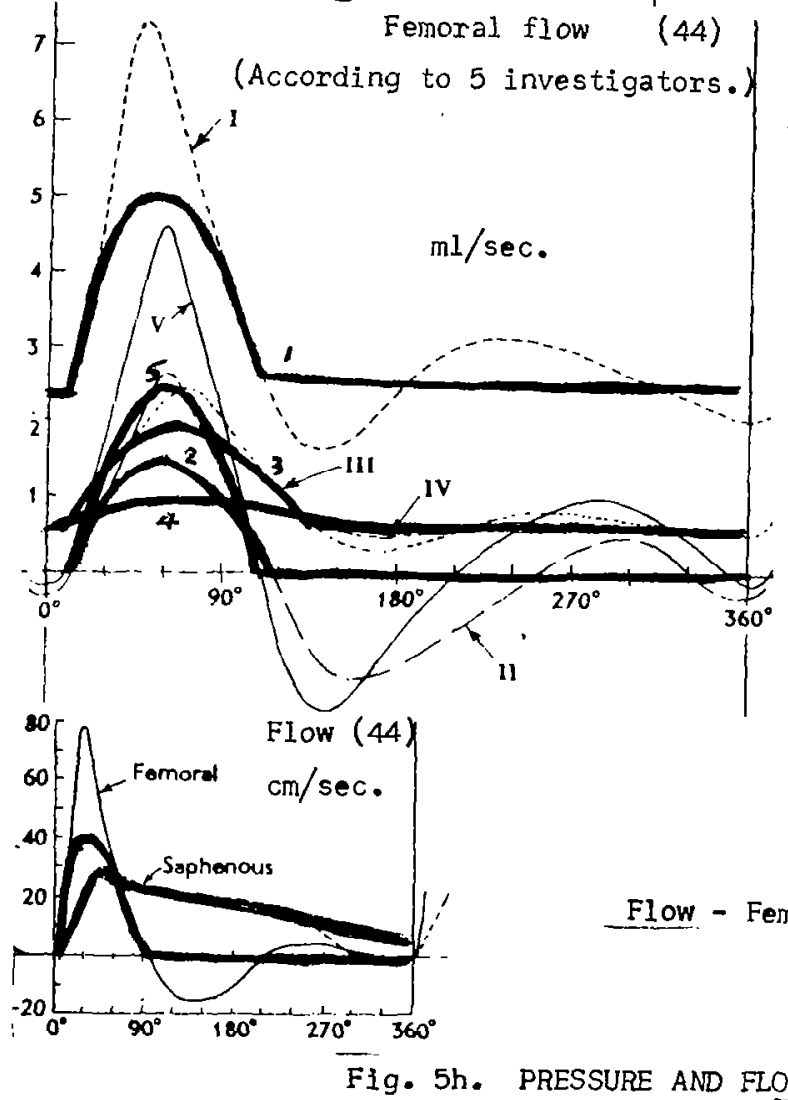

(52)

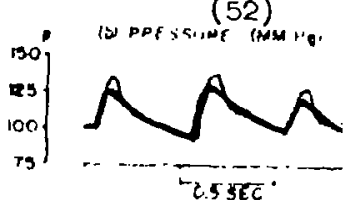

$\int_{10}^{\infty} \wedge \Lambda$

Combined Records
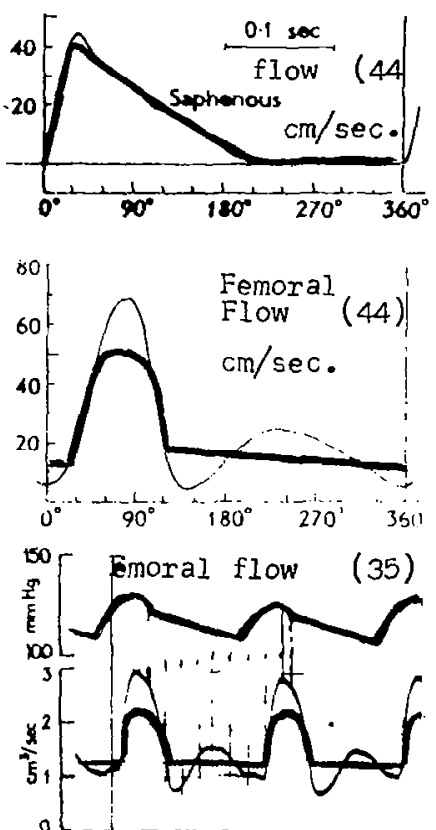

Flow - Femoral Artery
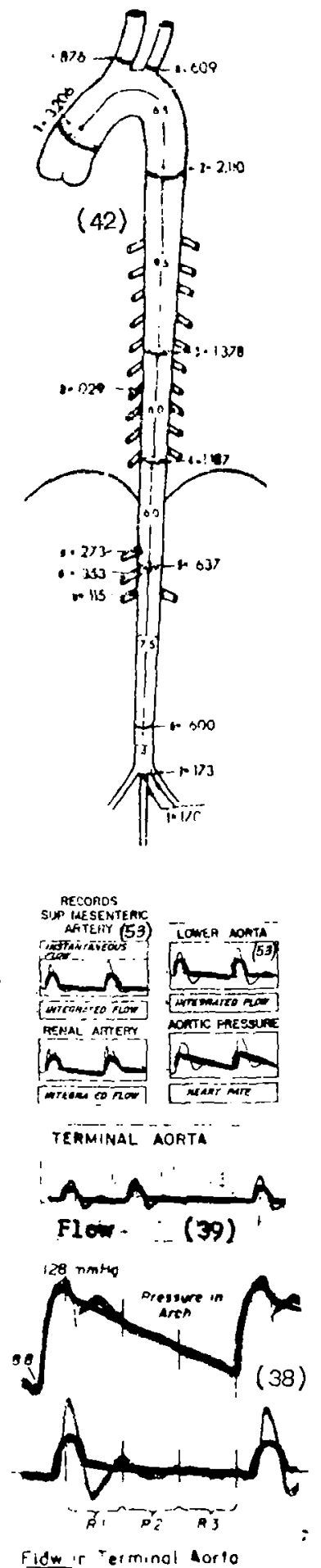

Terminal Aorta 


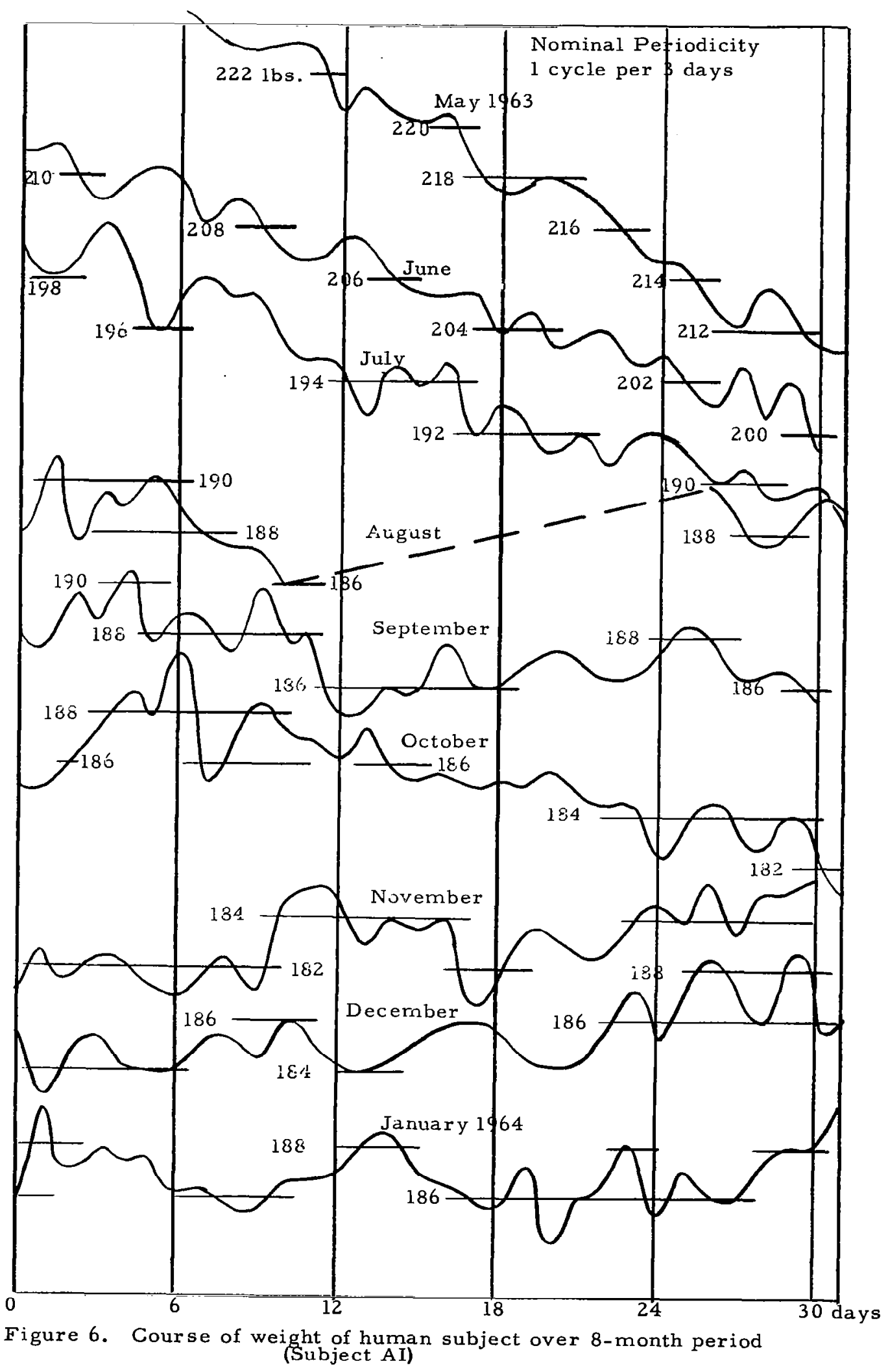



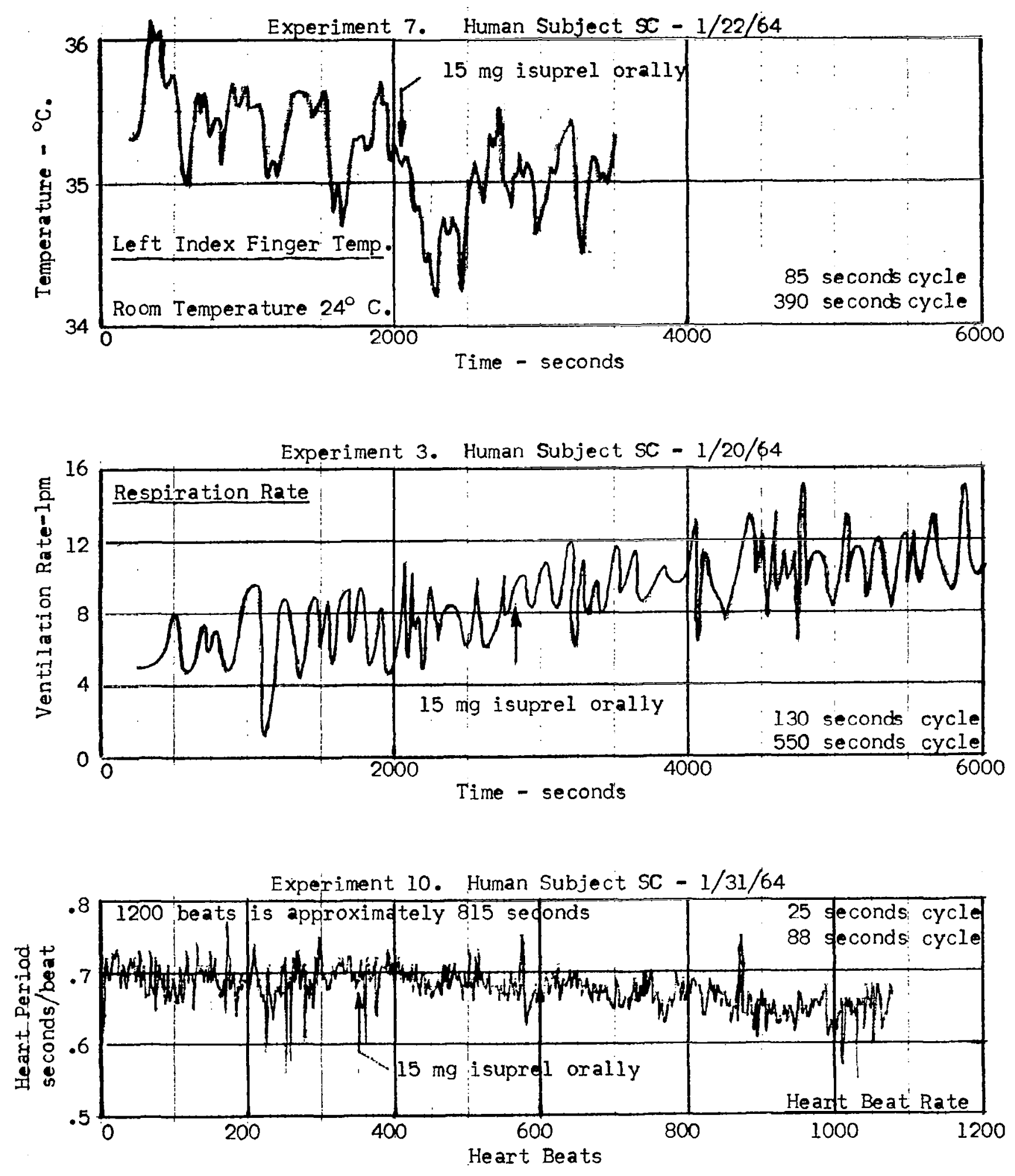

Fig. 7. Preliminary dynamic data on three oscillators to assess the background sensitivity for drug effects 


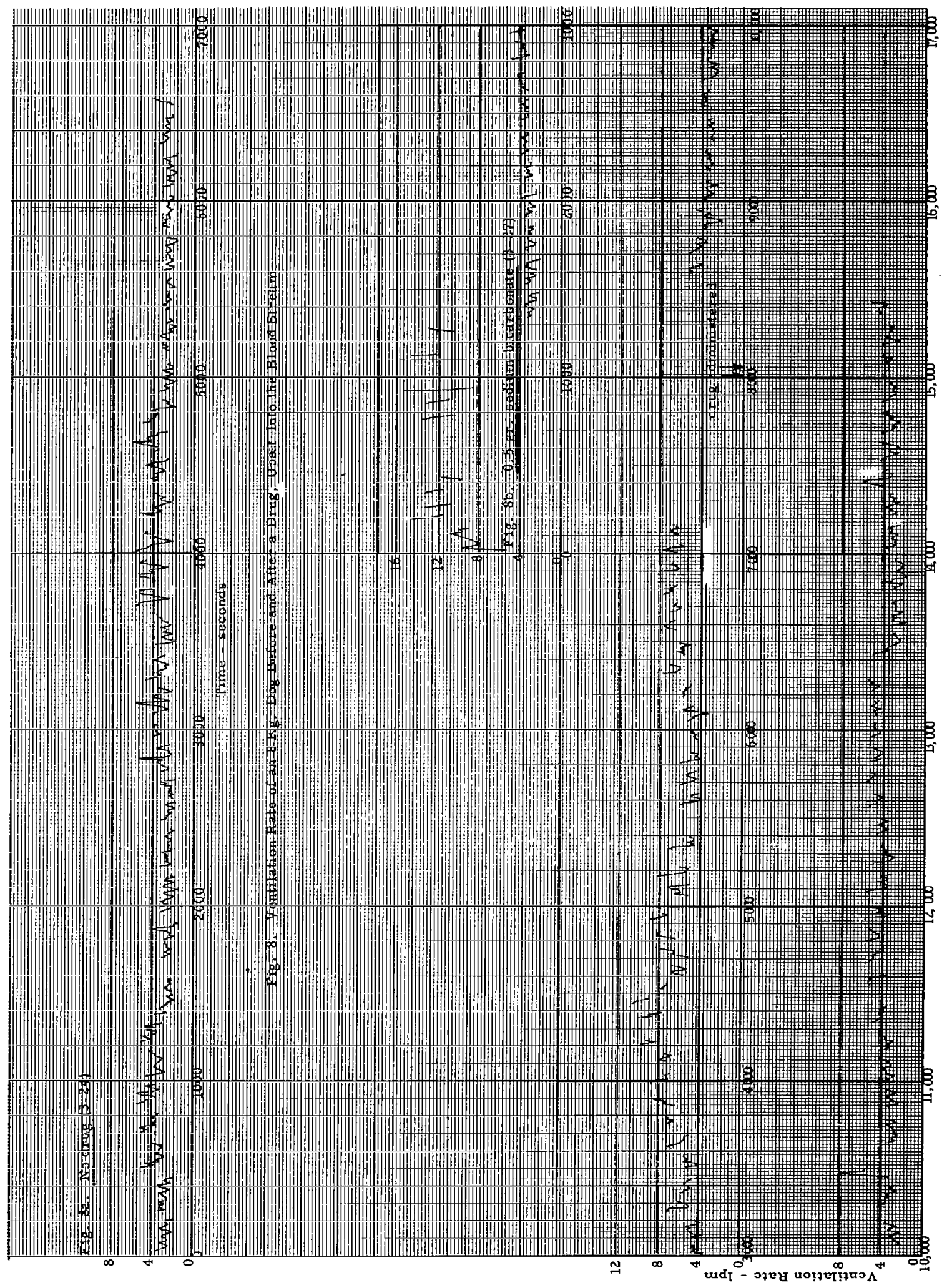




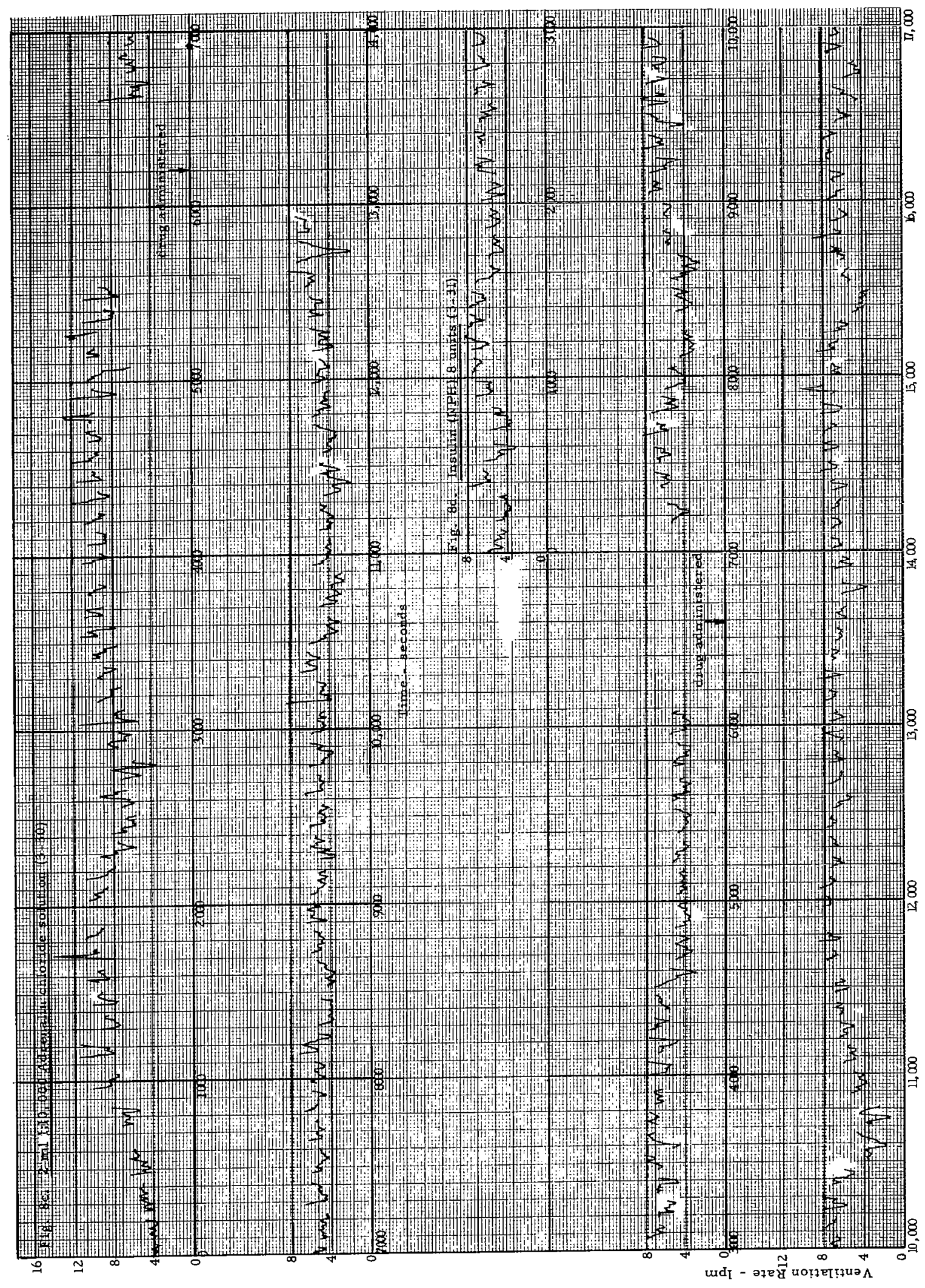




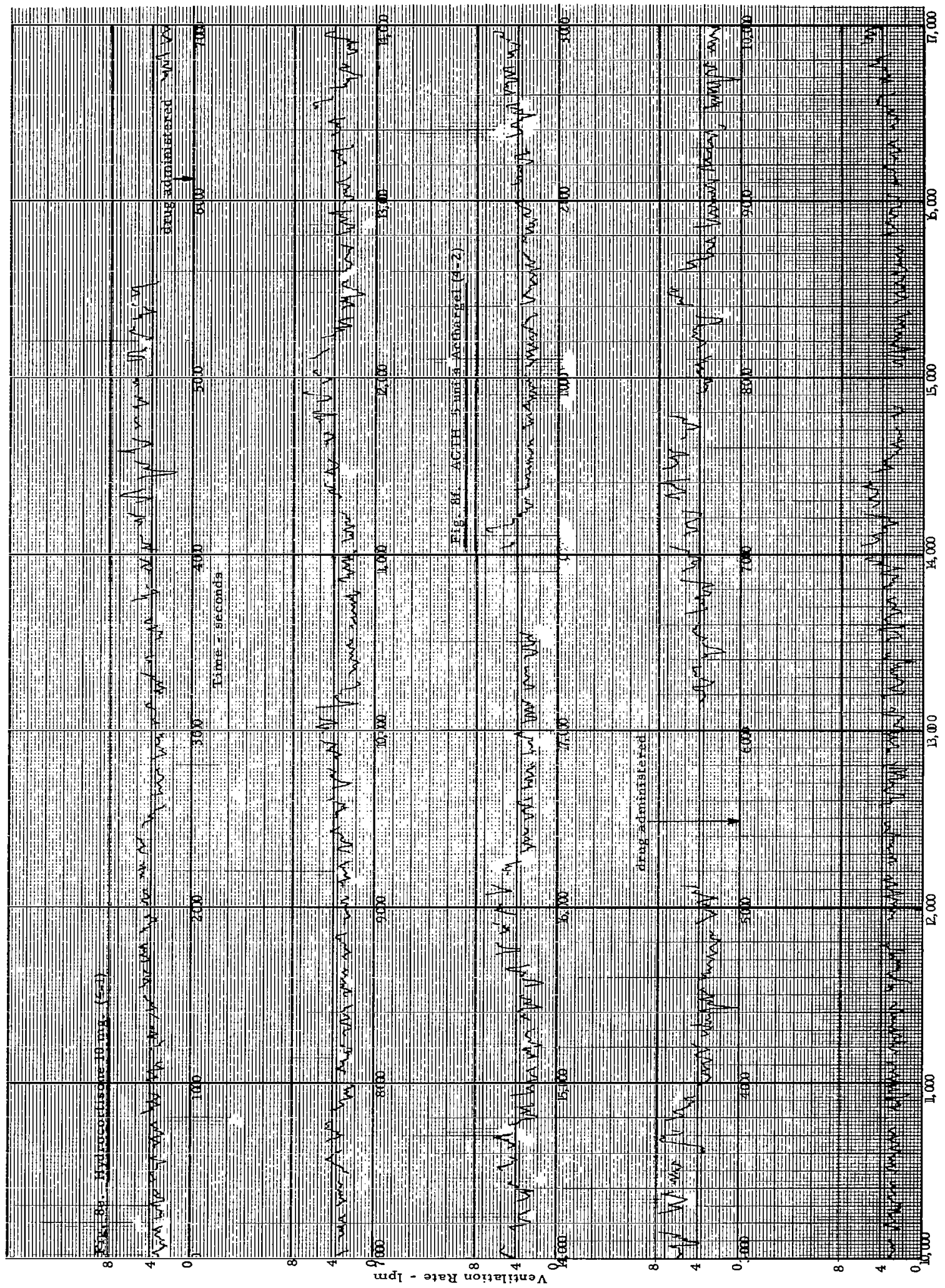




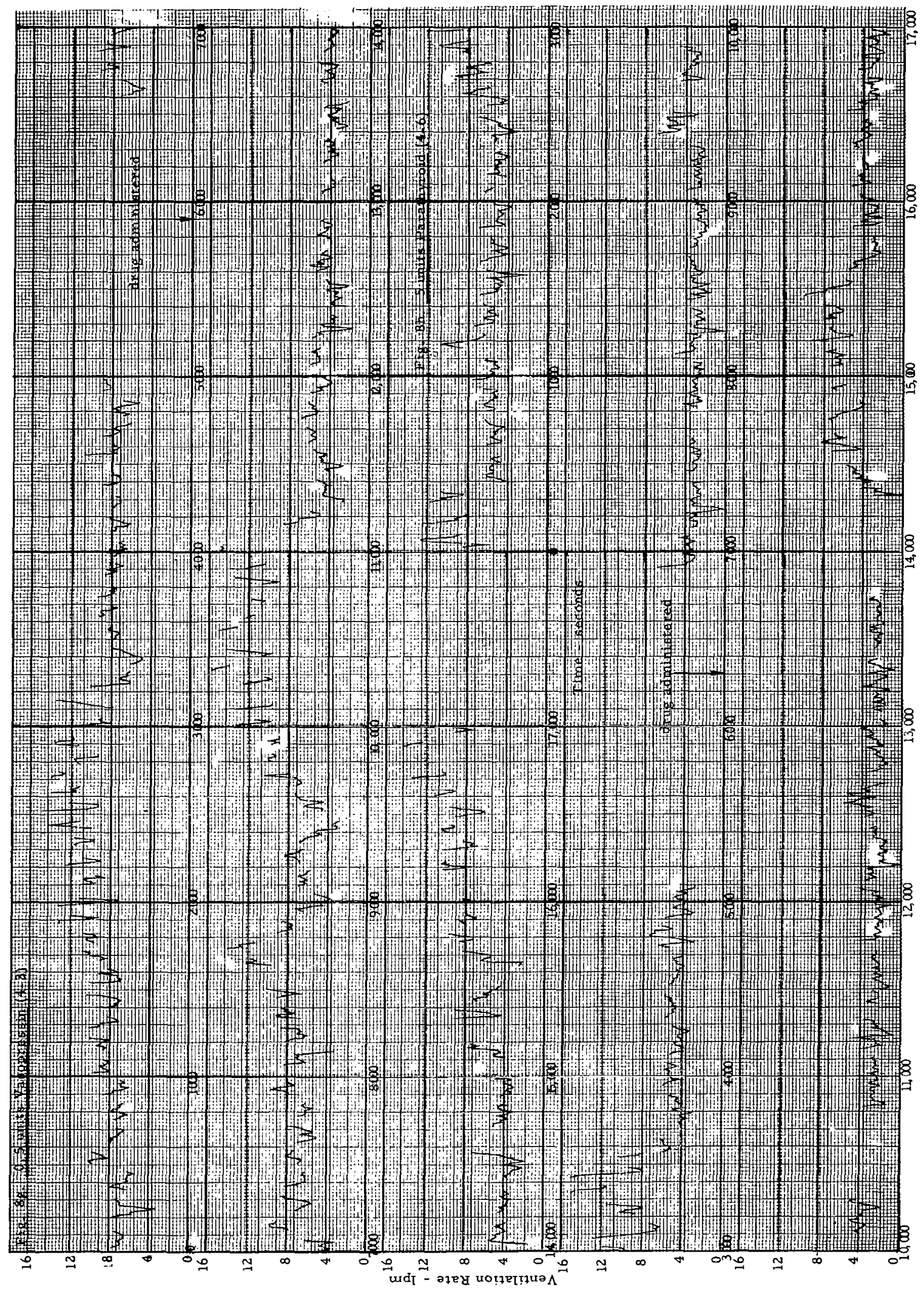




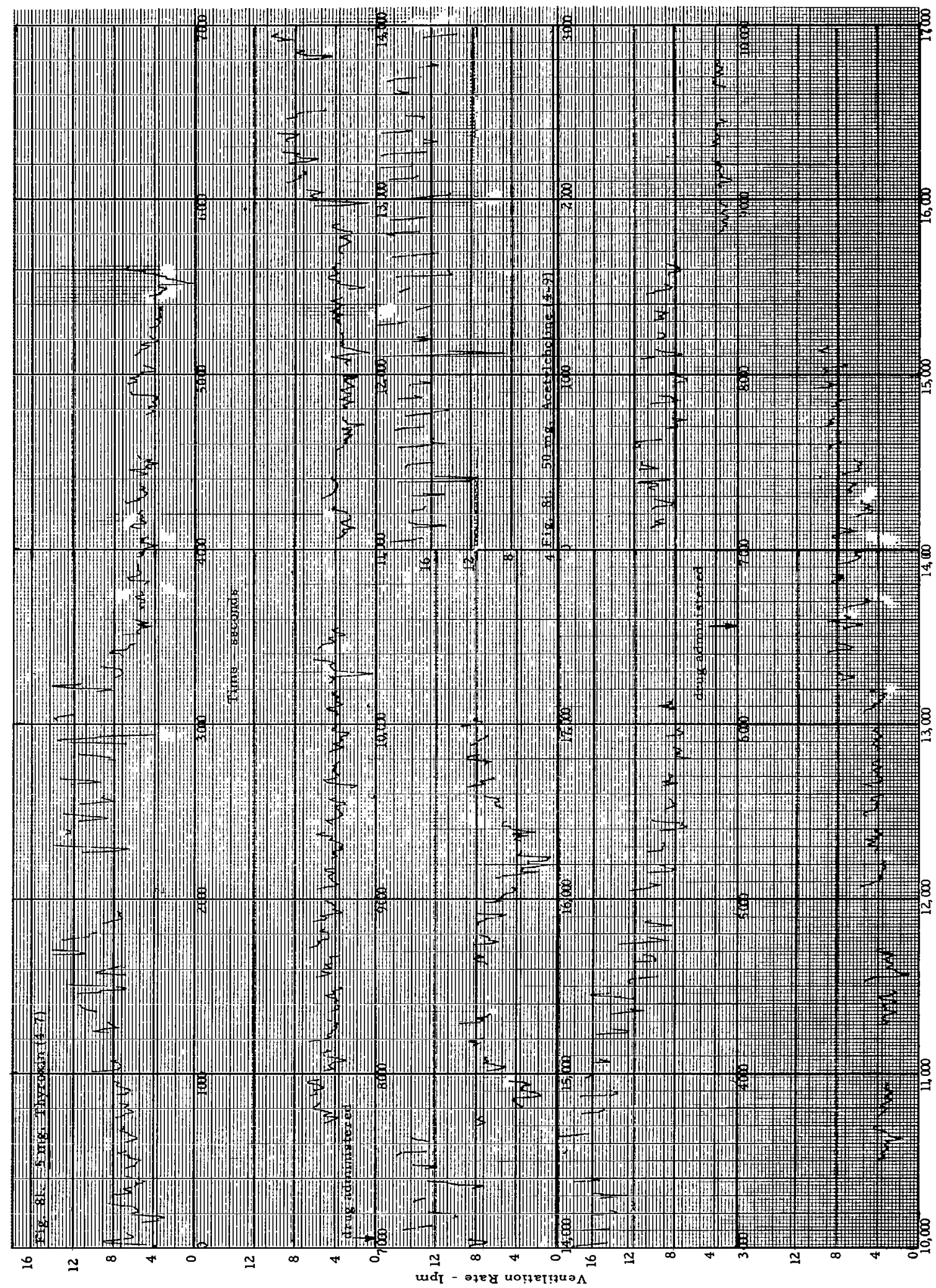




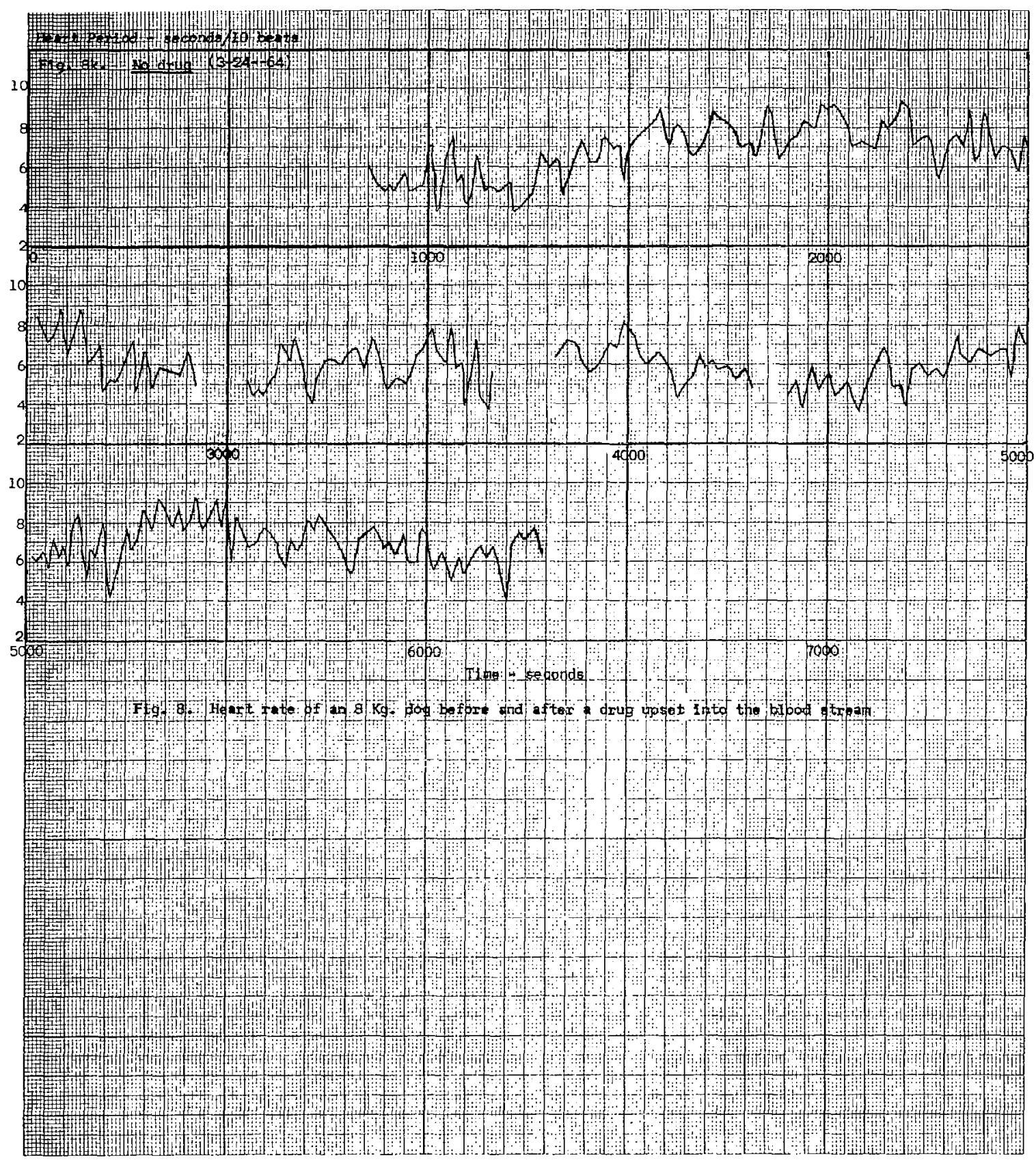




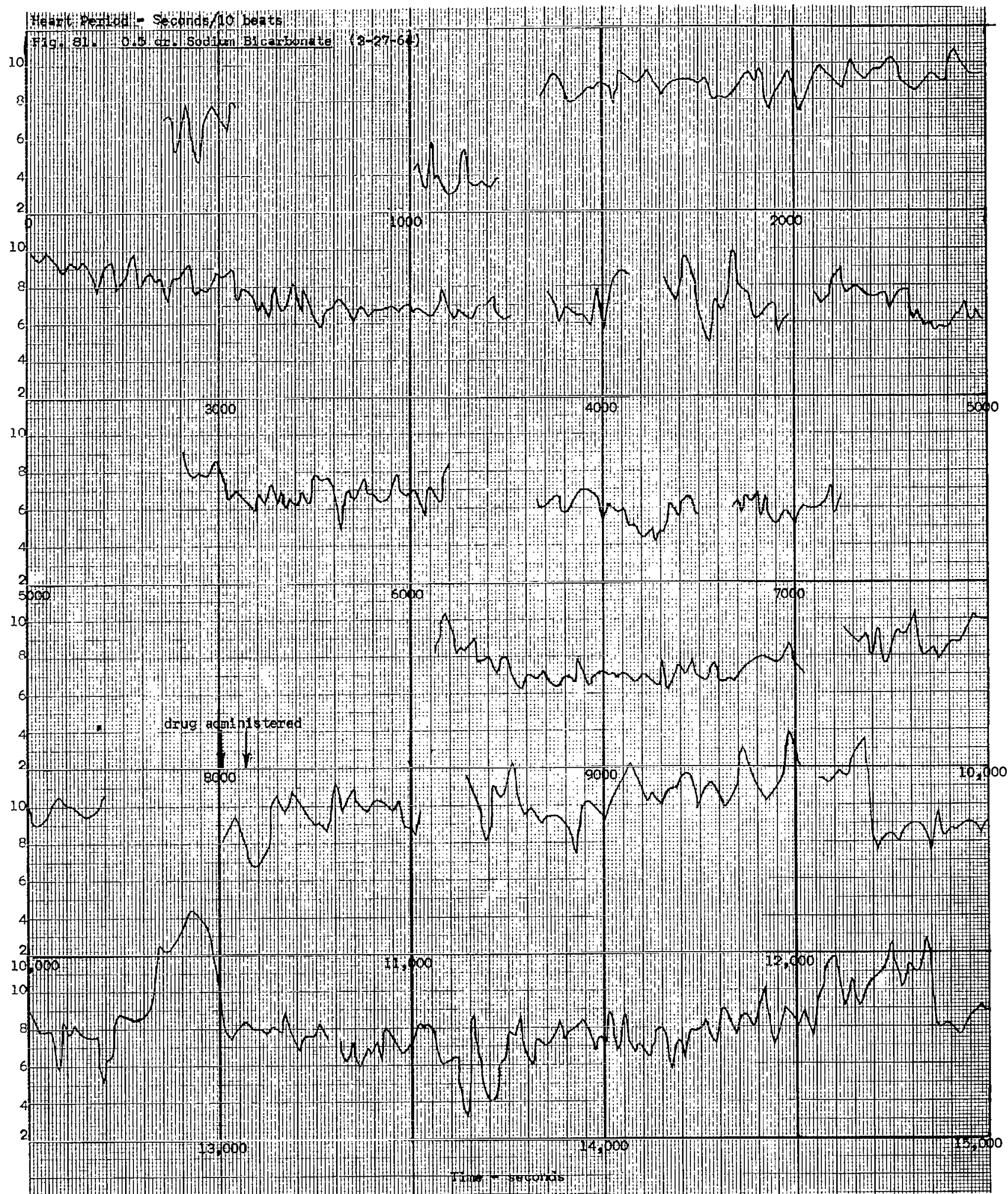




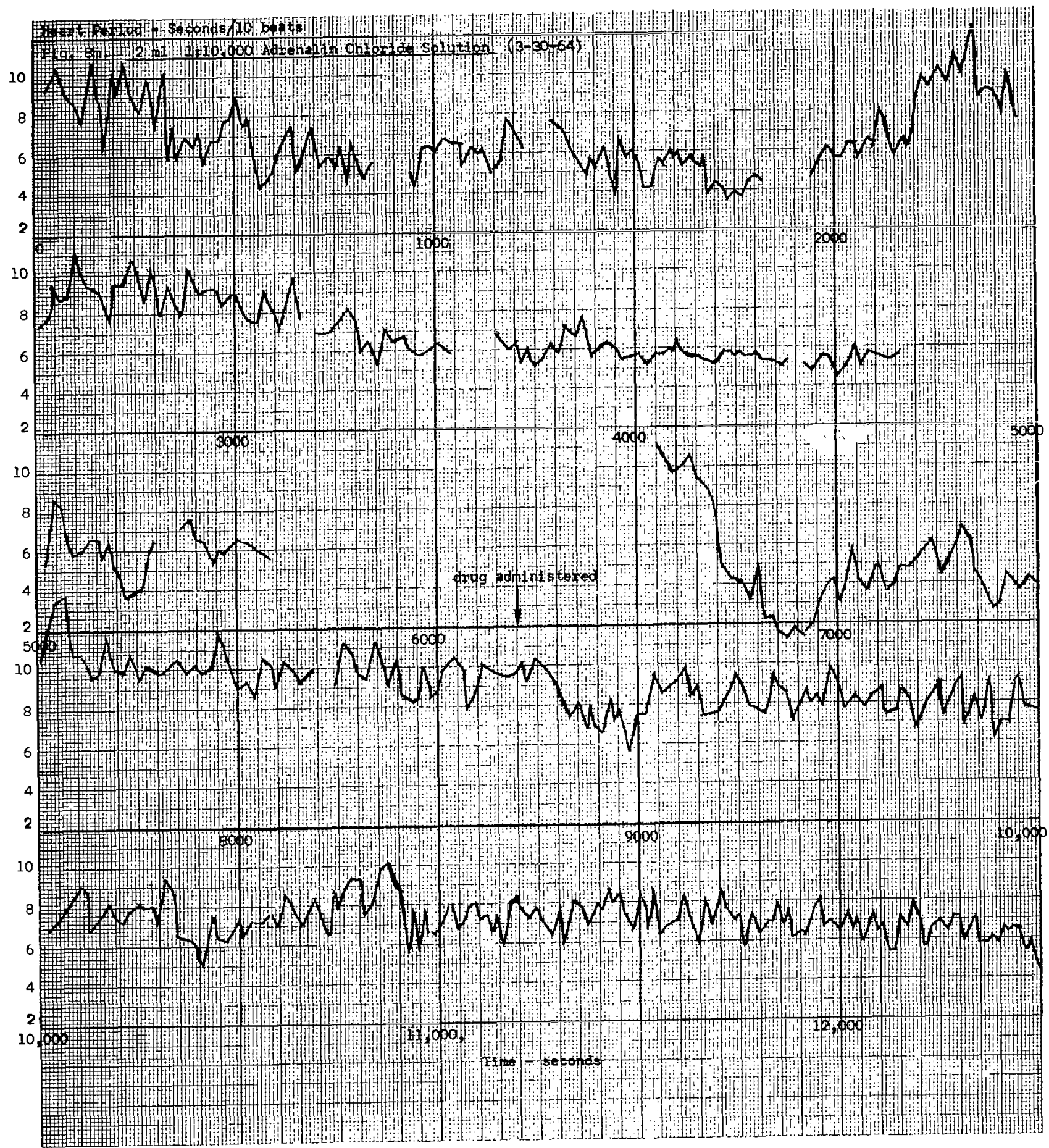




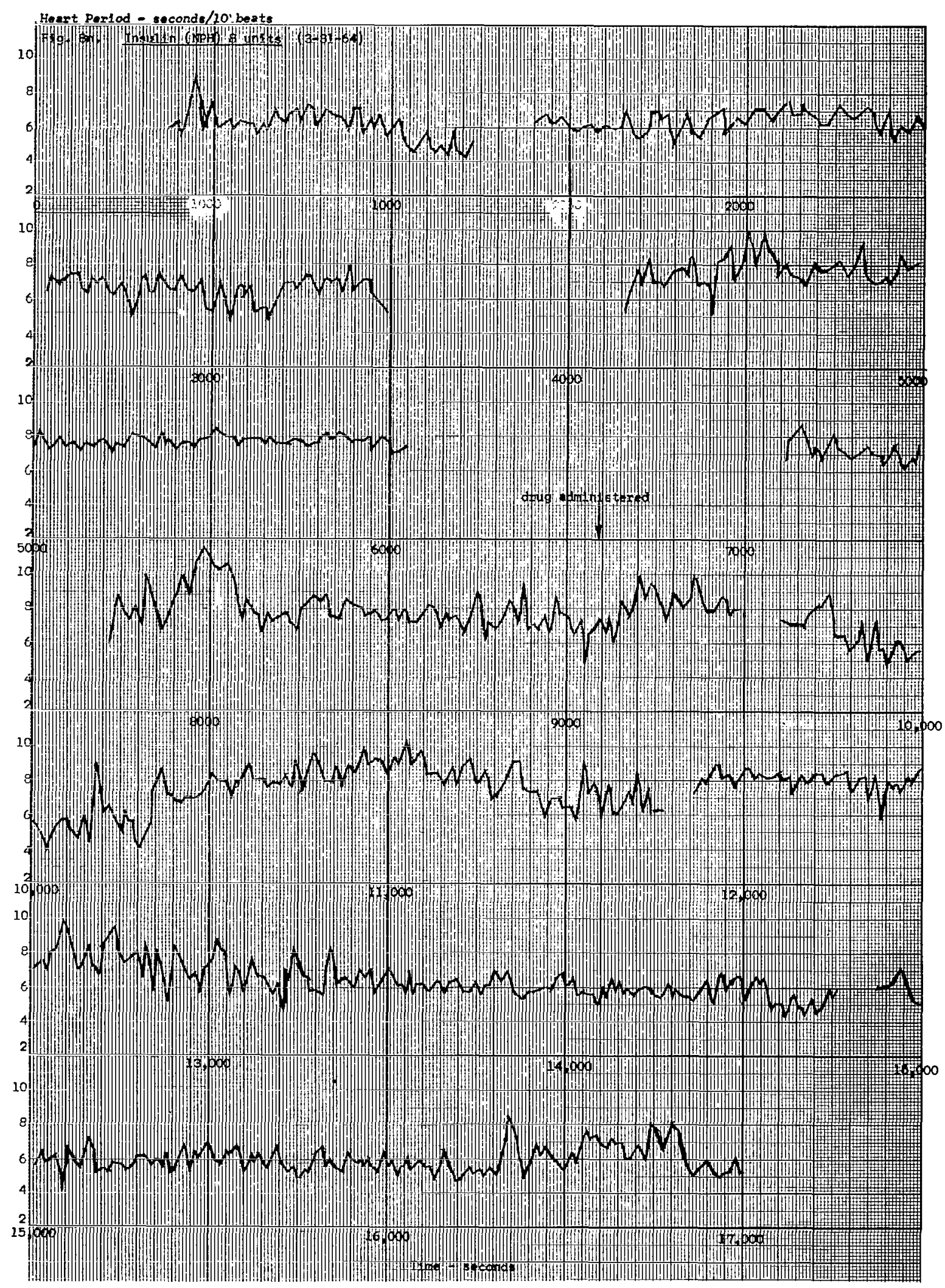




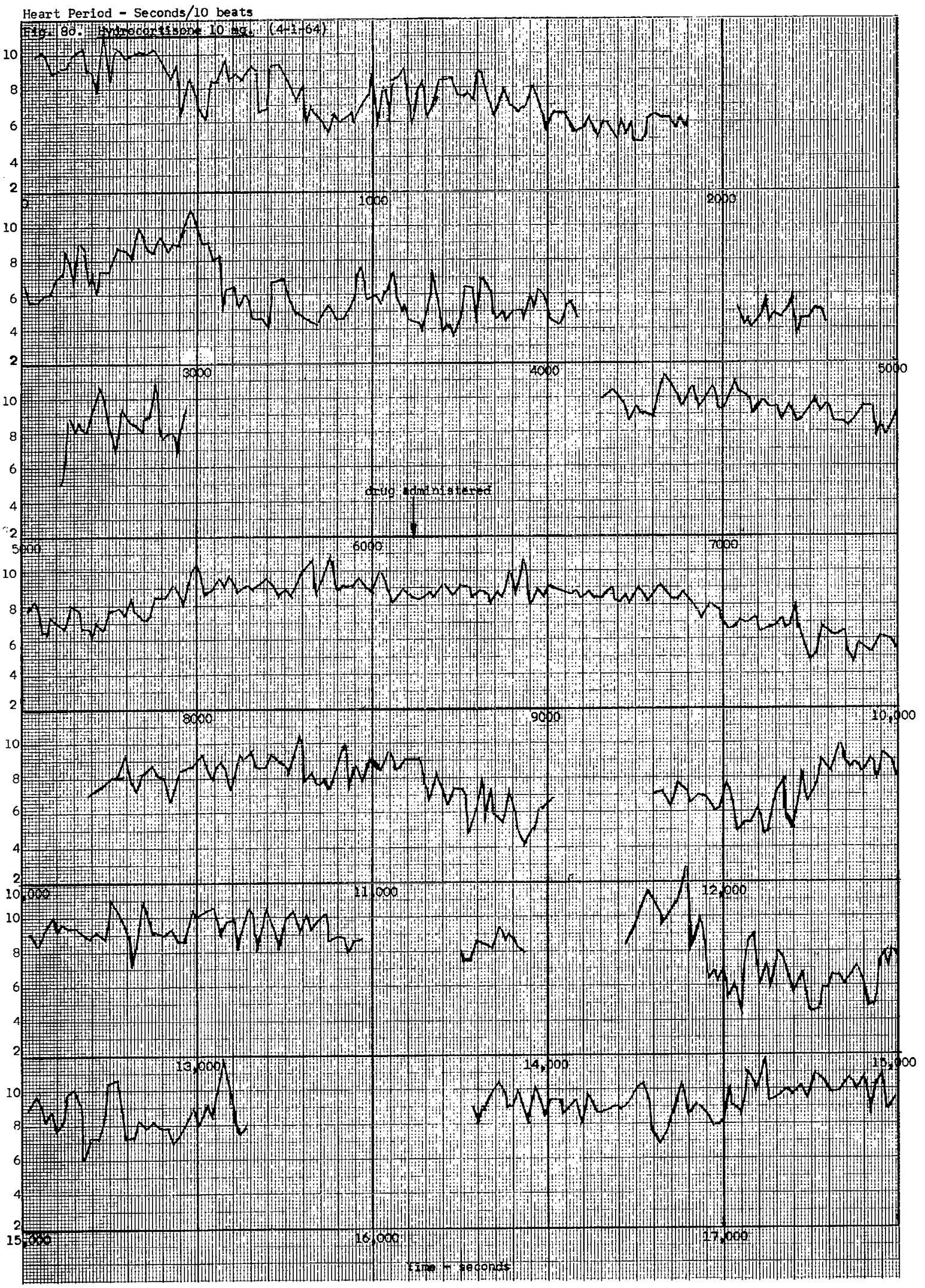




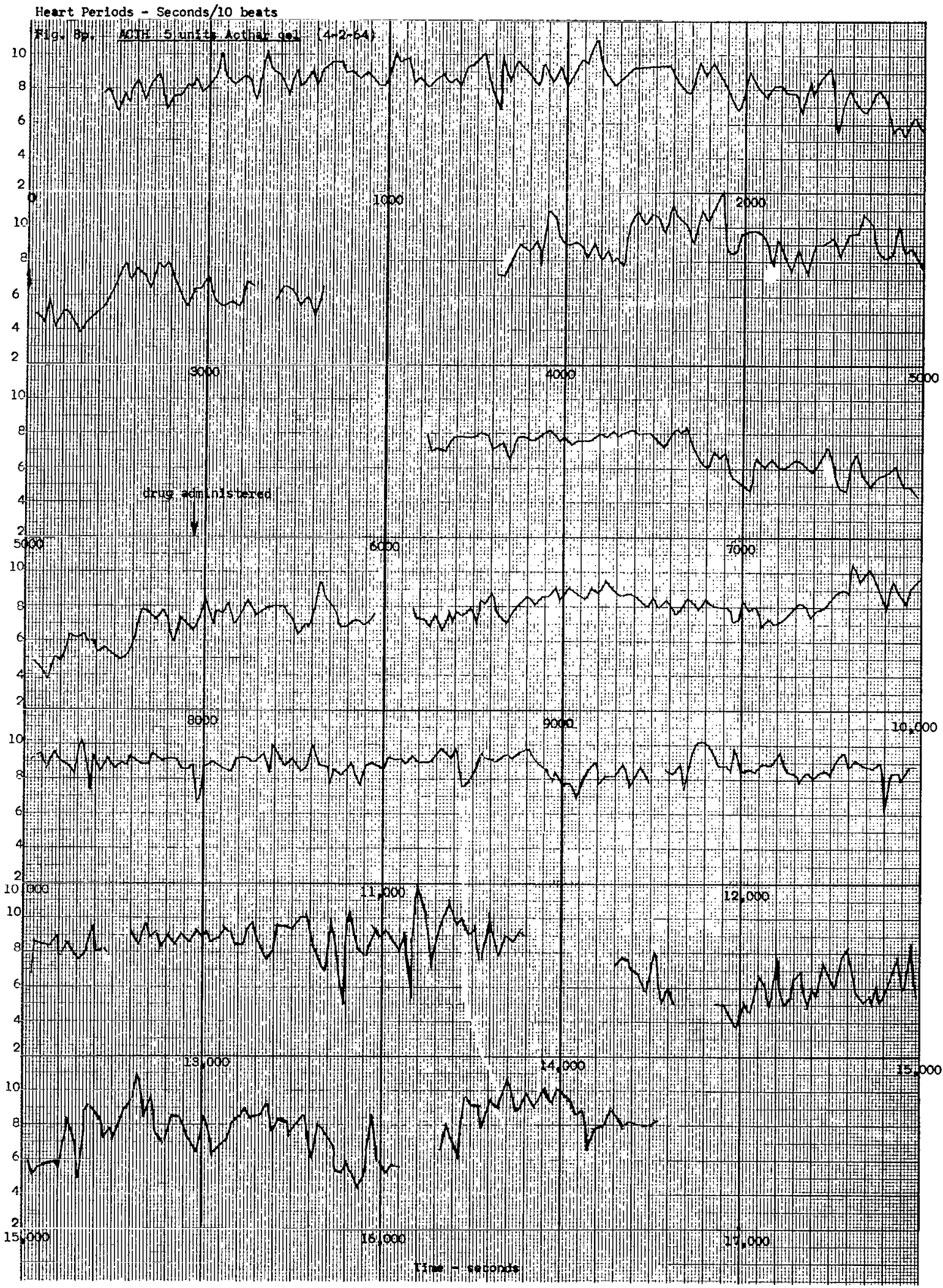




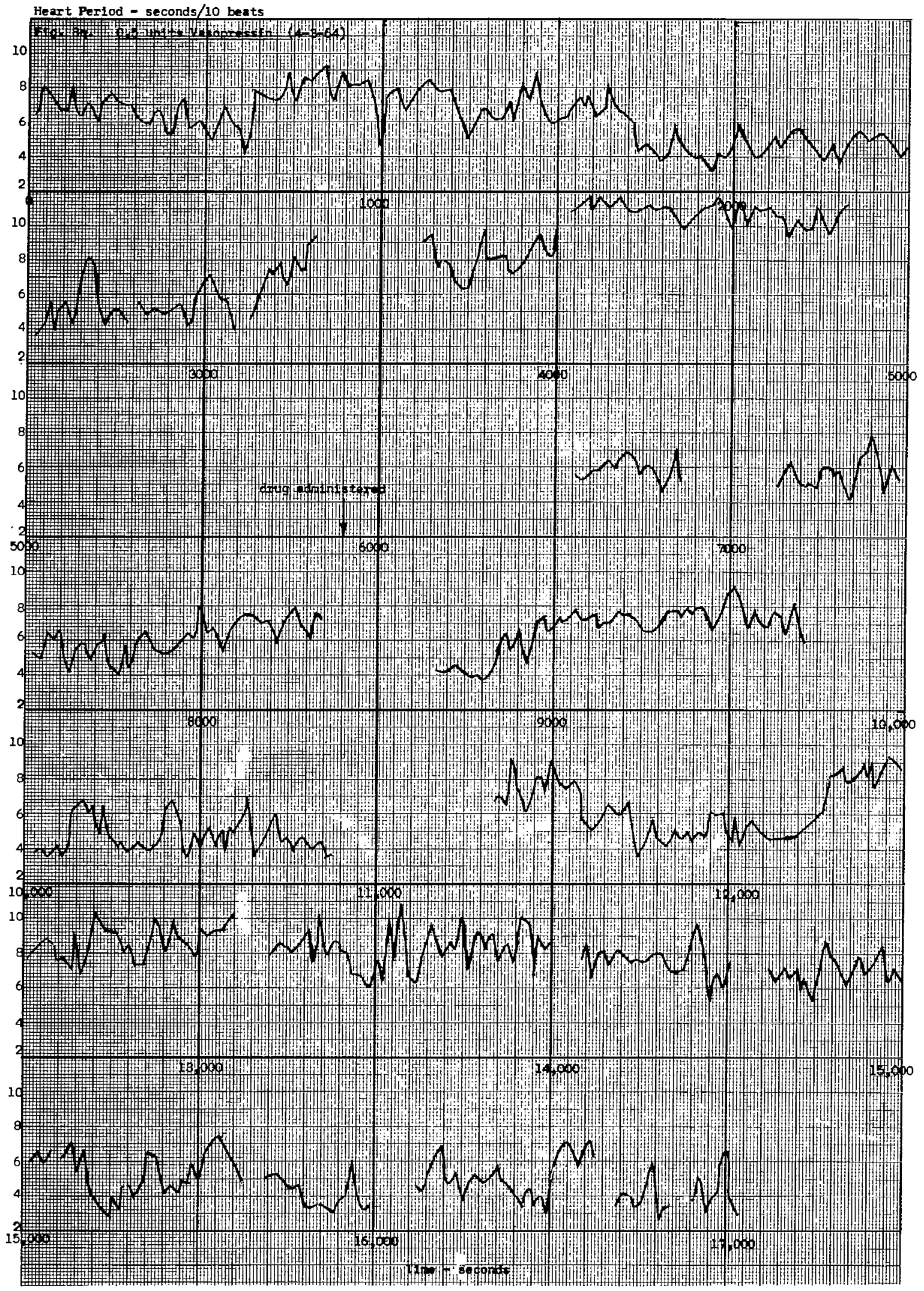




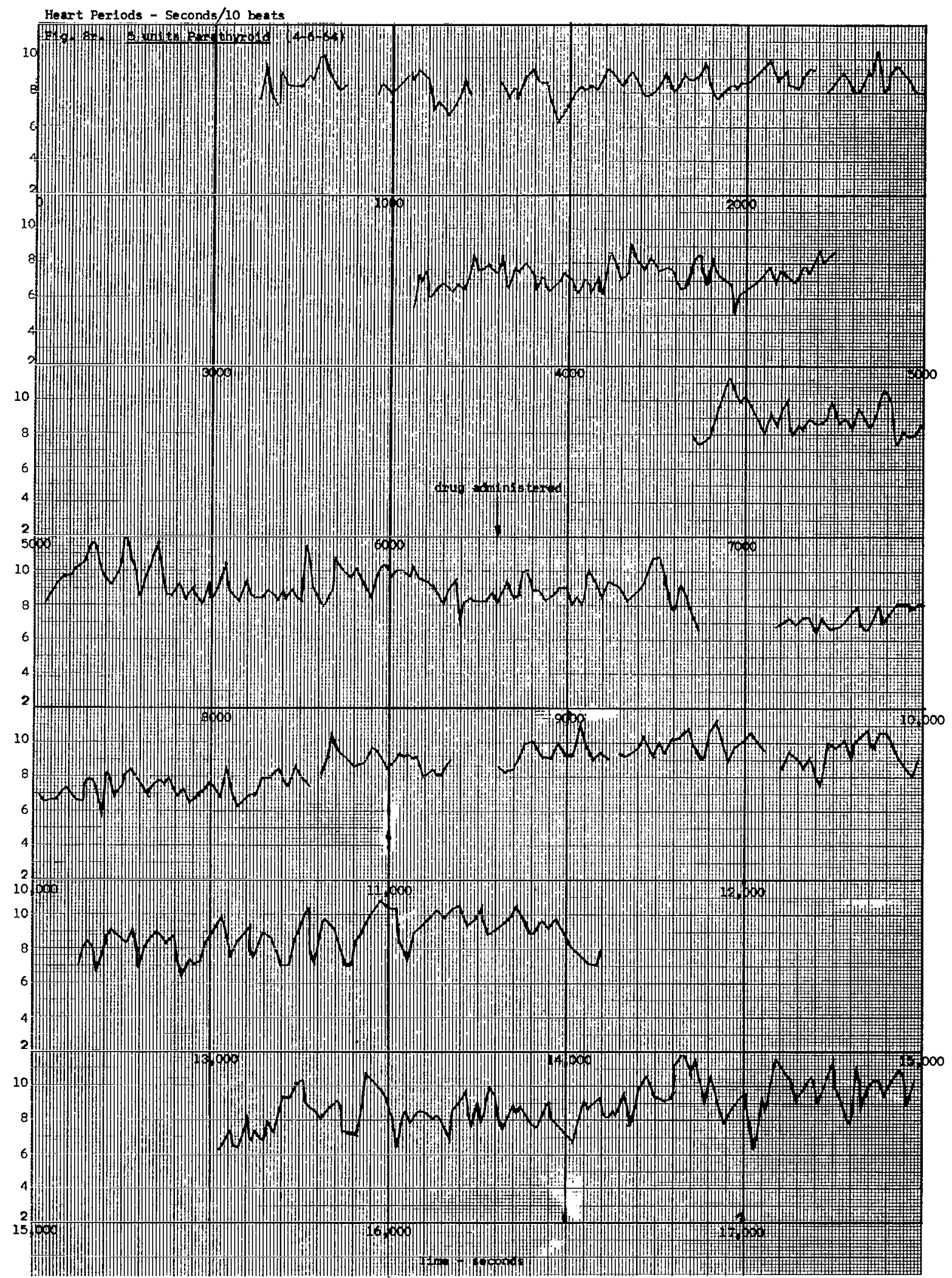




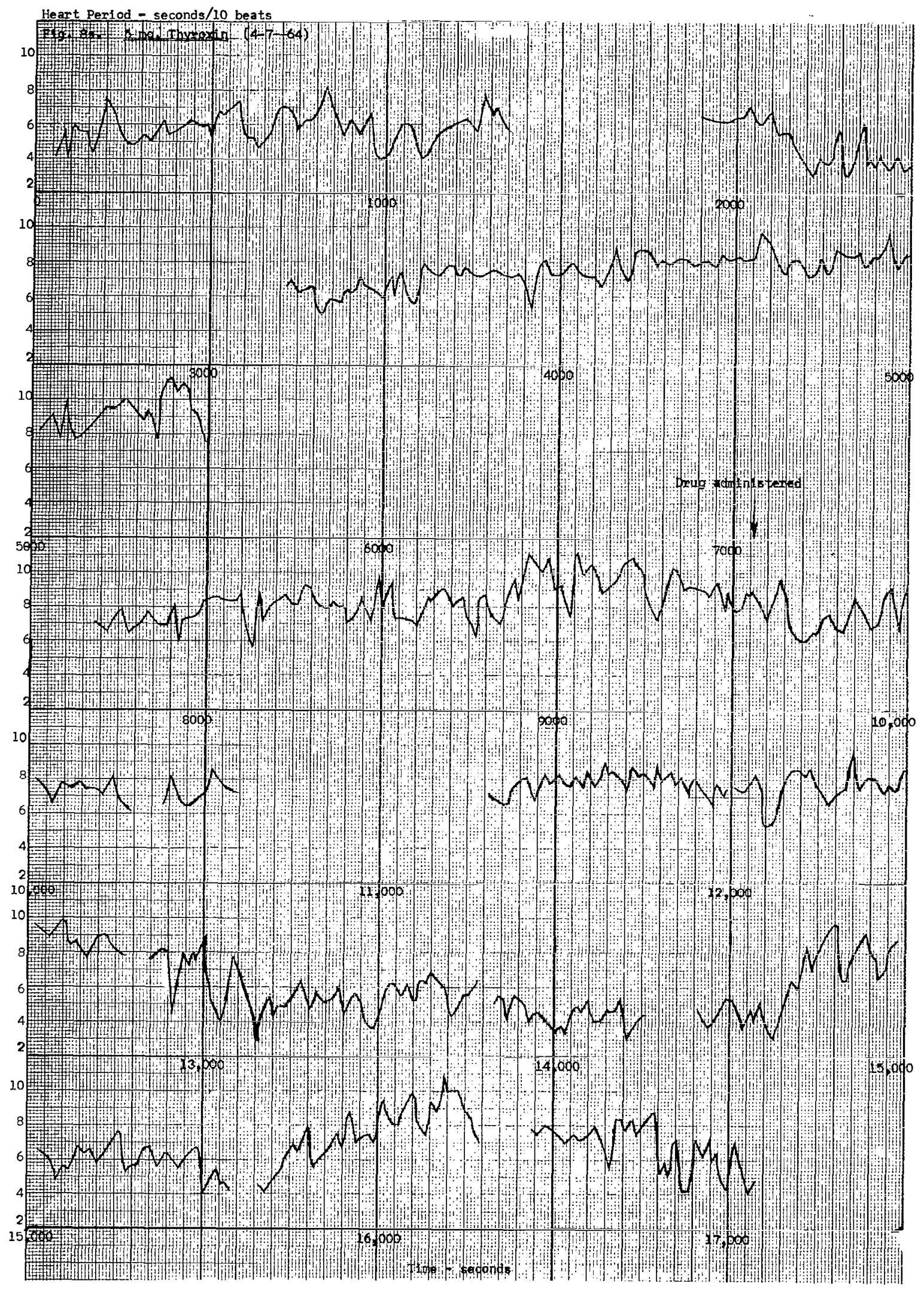




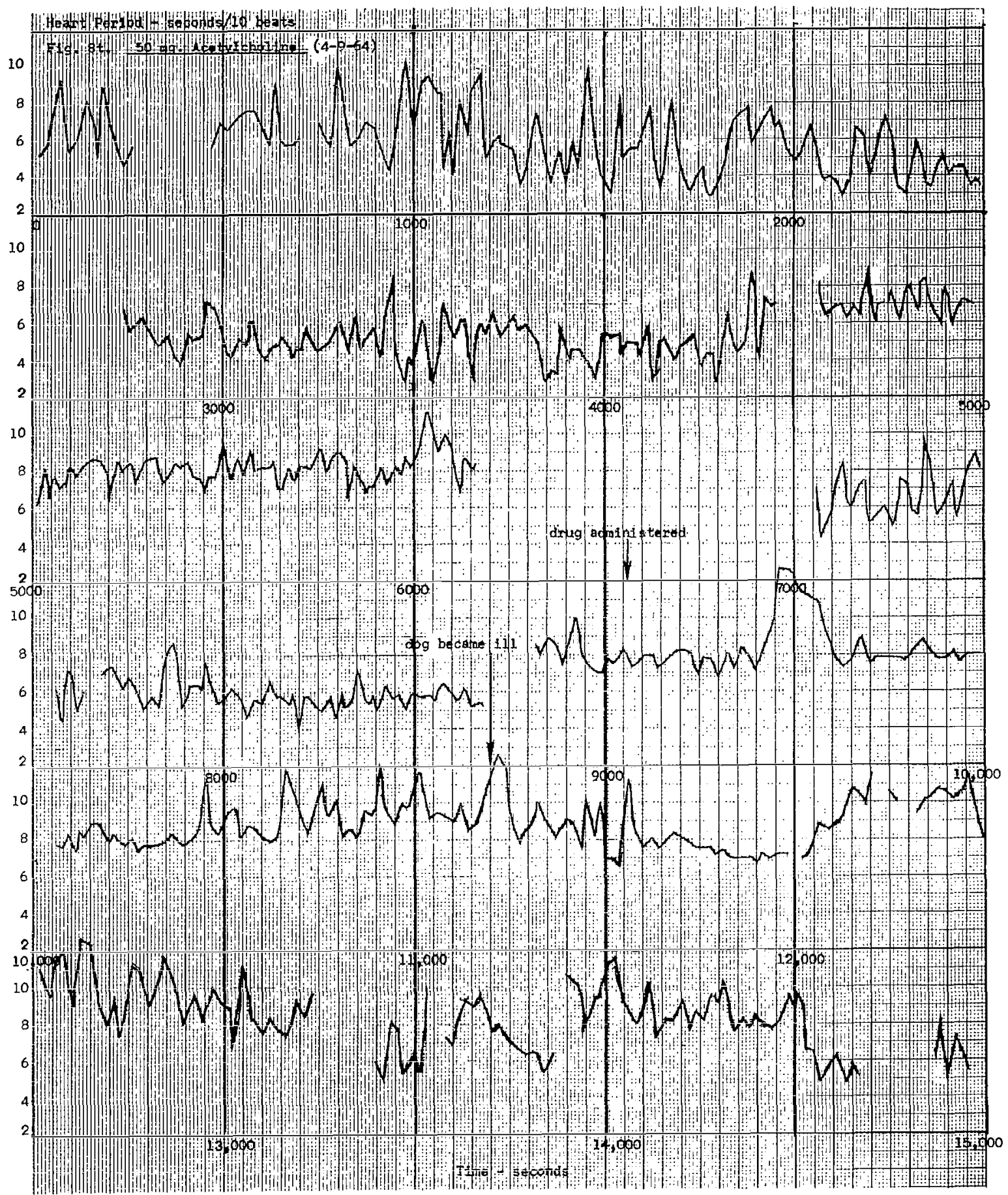

NASA-Langley, $1965 \quad \mathrm{CR}-219$ 